\title{
Weatherization and Indoor Air Quality: Measured Impacts in Single-family Homes under the Weatherization Assistance Program
}

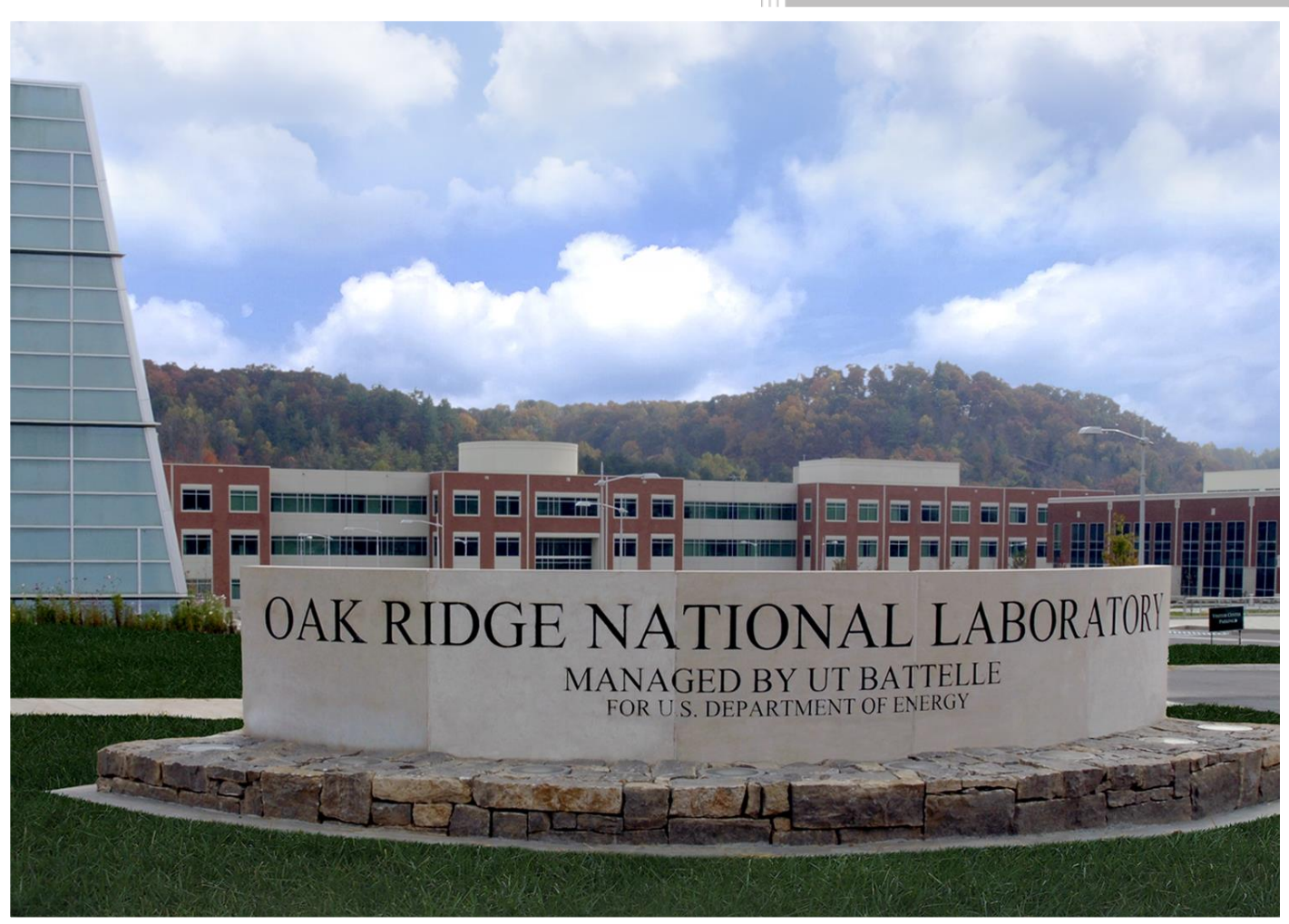

Approved for public release; distribution is unlimited.
Scott Pigg Dan Cautley Paul Francisco Beth Hawkins Terry Brennan

September 2014 


\section{DOCUMENT AVAILABILITY}

Reports produced after January 1, 1996, are generally available free via US Department of Energy (DOE) SciTech Connect.

Website http://www.osti.gov/scitech/

Reports produced before January 1, 1996, may be purchased by members of the public from the following source:

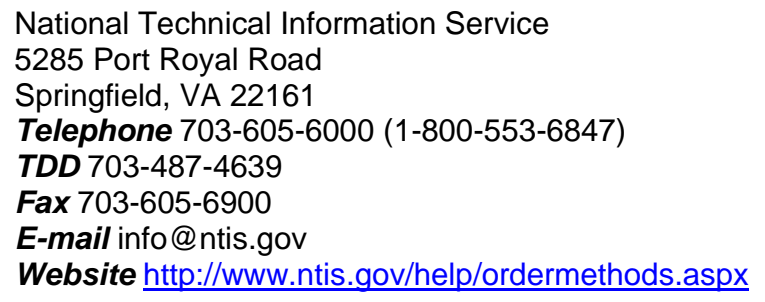

National Technical Information Service

5285 Port Royal Road

Springfield, VA 22161

Telephone 703-605-6000 (1-800-553-6847)

TDD 703-487-4639

Fax 703-605-6900

E-mail info@ntis.gov

Website http://www.ntis.gov/help/ordermethods.aspx

Reports are available to DOE employees, DOE contractors, Energy Technology Data Exchange representatives, and International Nuclear Information System representatives from the following source:

Office of Scientific and Technical Information

PO Box 62

Oak Ridge, TN 37831

Telephone 865-576-8401

Fax 865-576-5728

E-mail reports@osti.gov

Website http://www.osti.gov/contact.html

This report was prepared as an account of work sponsored by an agency of the United States Government. Neither the United States Government nor any agency thereof, nor any of their employees, makes any warranty, express or implied, or assumes any legal liability or responsibility for the accuracy, completeness, or usefulness of any information, apparatus, product, or process disclosed, or represents that its use would not infringe privately owned rights. Reference herein to any specific commercial product, process, or service by trade name, trademark, manufacturer, or otherwise, does not necessarily constitute or imply its endorsement, recommendation, or favoring by the United States Government or any agency thereof. The views and opinions of authors expressed herein do not necessarily state or reflect those of the United States Government or any agency thereof.

ORNL Principal Investigator

Dr. Bruce Tonn

\section{Evaluation Team Task Manager Scott Pigg}


Environmental Sciences Division

\section{WEATHERIZATION AND INDOOR AIR QUALITY: MEASURED IMPACTS IN SINGLE-FAMILY HOMES UNDER THE WEATHERIZATION ASSISTANCE PROGRAM}

Scott Pigg, Energy Center of Wisconsin Dan Cautley, Energy Center of Wisconsin Paul Francisco, University of Illinois

Beth Hawkins, Oak Ridge National Laboratory

Terry Brennan, Camroden Associates

Date Published: September 2014

Prepared by

OAK RIDGE NATIONAL LABORATORY

Oak Ridge, Tennessee 37831-6283

managed by

UT-BATTELLE, LLC

for the

US DEPARTMENT OF ENERGY

under contract DE-AC05-00OR22725 



\section{CONTENTS}

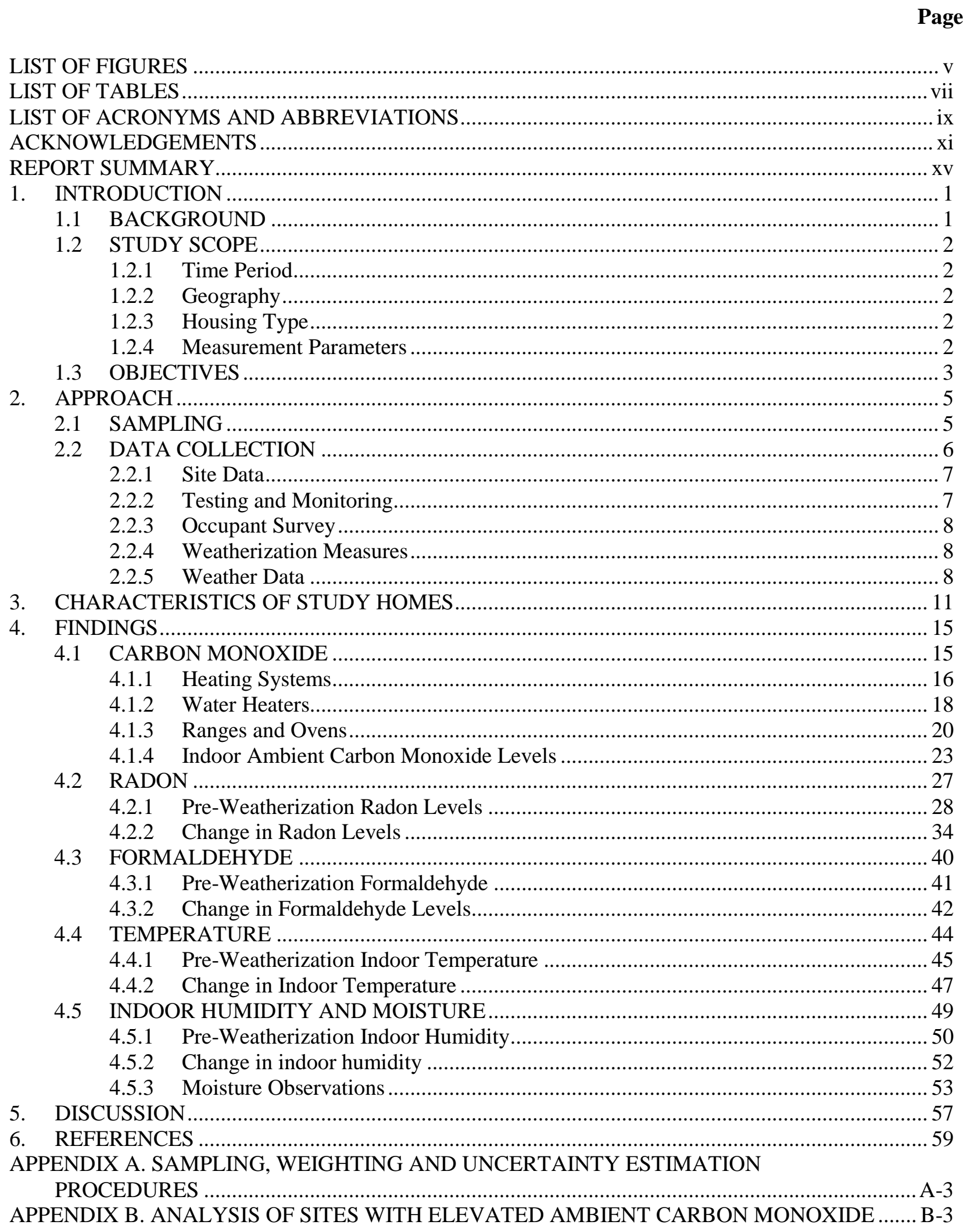


APPENDIX C. RADON LITERATURE REVIEW

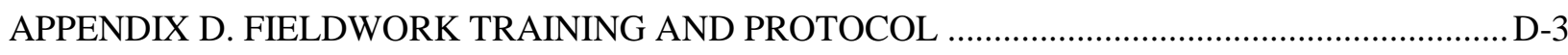

APPENDIX E. FIELD TECHNICIAN DATA COLLECTION FORM …........................................ E-3

APPENDIX F. PICK-UP TECHNICIAN DATA COLLECTION FORM …....................................

APPENDIX G. OCCUPANT SURVEY INSTRUMENT ....................................................................

APPENDIX H. LOCAL WEATHERIZATION AGENCY SCRIPTS AND FORMS .......................... H-3

APPENDIX I. HOUSEHOLD RECRUITMENT SCHEDULING SCRIPTS AND INFORMATION

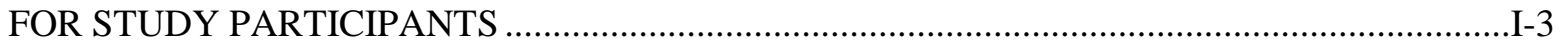

APPENDIX J. FIELD DEPLOYMENT SCHEDULE ……............................................................... J-3 


\section{LIST OF FIGURES}

Figures $\quad$ Page

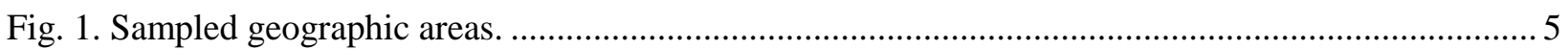

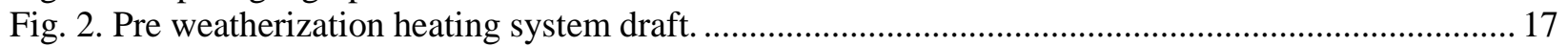

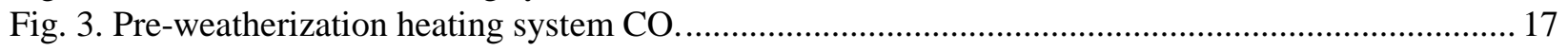

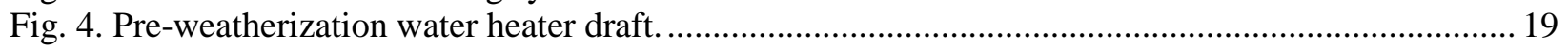

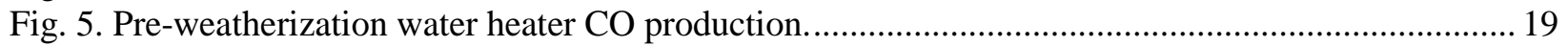

Fig. 6. Pre-weatherization carbon monoxide production by range burners. ......................................... 21

Fig. 7. Pre- versus post-weatherization range burner carbon monoxide ............................................... 21

Fig. 8. Pre-weatherization carbon monoxide production by ovens........................................................ 22

Fig. 9. Pre- versus post-weatherization oven carbon monoxide. .......................................................... 23

Fig. 10. Five-minute indoor ambient CO data for Site 9626-I20, with expanded view of January

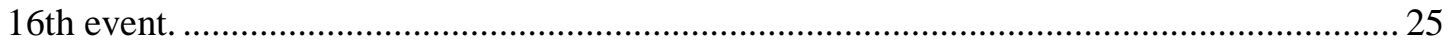

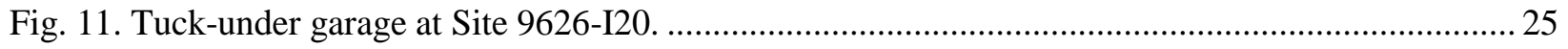

Fig. 12. Indoor ambient CO at Site 5431-I05 on January 25, 2011 .................................................. 26

Fig. 13. Daily range and median indoor ambient CO level at Site 5431-I05 ......................................... 27

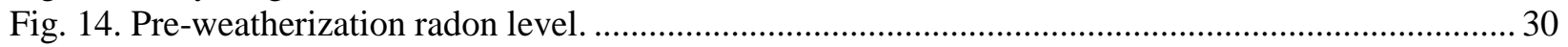

Fig. 15. Above-grade versus foundation-level radon level (pre-weatherization). ................................. 32

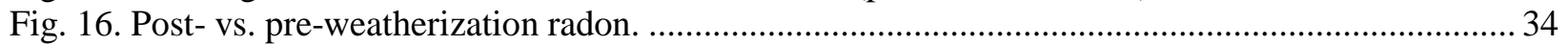

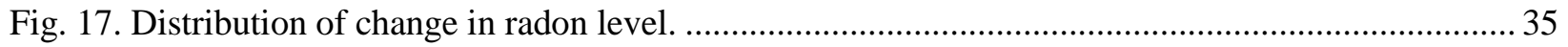

Fig. 18. Median change in control group radon level vs. change in outdoor temperature.......................... 37

Fig. 19. Distribution of pre-weatherization formaldehyde level............................................................ 41

Fig. 20. Pre- versus post-weatherization indoor formaldehyde level.........................................................4

Fig. 21. Change in formaldehyde level vs. change in relative humidity................................................. 44

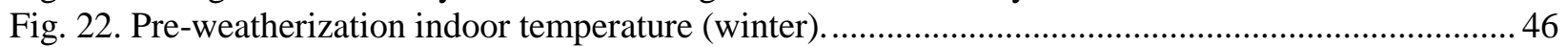

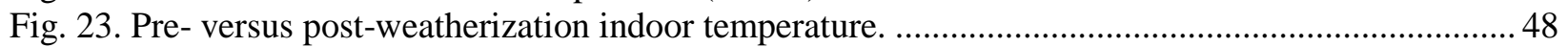

Fig. 24. Change in indoor temperature by pre-weatherization temperature quintile. ............................. 49

Fig. 25. Pre-weatherization indoor relative humidity (winter). ....................................................... 51

Fig. 26. Pre-weatherization indoor dew-point versus average winter outdoor temperature...................... 51

Fig. 27. Pre- versus post-weatherization indoor relative humidity................................................... 52

Fig. 28. Frequency of recorded moisture problems for foundations (left) and above grade (right)............53

Fig. 29. Incidence of recorded foundation moisture problems for sites with the same technician preand post-weatherization (with $90 \%$ confidence intervals). .................................................. 54

Fig. 30. Incidence of recorded above-grade moisture problems for sites with the same technician pre- and post-weatherization (with $90 \%$ confidence intervals) ........................................... 55

Fig. 31. Pre-weatherization recorded severity of water stains and mold for foundation spaces (left)

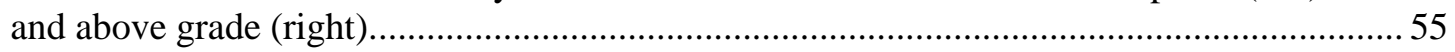

Fig. A.1. Super-PUMA classification by radon stratum. ….........................................................

Fig. C.1 Recommended radon reference levels by country. ........................................................ C-8

Fig. C. 2 Hourly radon measurements made by Arthur Scott in a basement in New York State from November 25, 1992 to February 13, 1993 (Scott 1993).................................................... C-11

Fig. C. 3 Normalized radon concentrations for the 20 houses in the EPA seasonal variation study in Montana (EPA 1986). 17 of the houses showed winter peaking radon concentrations. .... C-12

Fig. C.4 Wide variation in radon concentrations over short time periods (Dudney 1992)................... C-13

Fig. C.5 Most commonly used radon measurement devices and their characteristics (WHO 2009)...... C-14

Fig. C.6 Coefficient of variation (\%) for varying duration of continuous radon monitor measurements compared to annual average and four winter months in Montana (RoncaBatista 1988) 
Fig. C.8 Screening measurements vs. annual average living space radon levels in 78 Minnesota houses (Steck 1990).

Fig. C.9 Markers at $4 \mathrm{pCi} / \mathrm{L}$ for screening and annual average measurements for 528 houses (White 1990). C-18

Fig. C.10 Subsets of homes (in Figure C.9) with additional screening measurements averaged with first - measurements are denoted by the letter B for two, C for 3, D for 4, etc.

Fig. C.11 Summary of Monitoring Results by Type of Weatherization Procedure. (Dyess 1994) 


\section{LIST OF TABLES}

\section{Tables}

Page

Table 1. Selected home characteristics, study sample (weighted) and WAP population......................... 11

Table 2. Selected demographic characteristics, study sample (weighted) and WAP population. .............. 13

Table 3. Selected weatherization characteristic, study sample (weighted) and WAP population. ............. 14

Table 4. Pre-weatherization peak indoor ambient $\mathrm{CO}$ and $\mathrm{CO}$ persistence............................................2 24

Table 5. Pre- and post-weatherization peak indoor ambient CO and CO persistence, by group............... 24

Table 6. Estimated PY08 single-family WAP housing-unit proportions by radon zone and housing

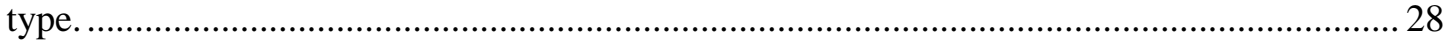

Table 7. Pre-weatherization radon level (lowest occupied level of home) . ............................................. 30

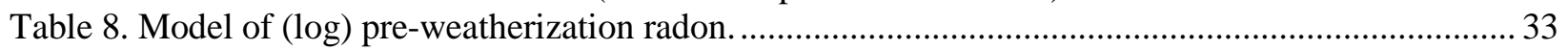

Table 9. Net change in radon level, by EPA zone and housing type (arithmetic means)......................... 35

Table 10. Net change in radon level for homes with pre-weatherization radon between 1 and

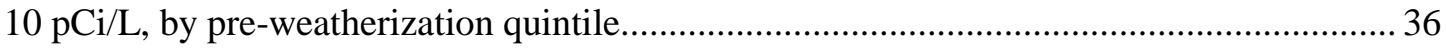

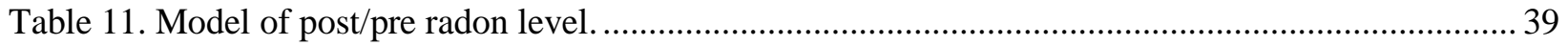

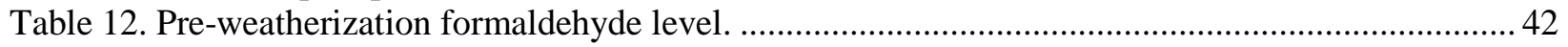

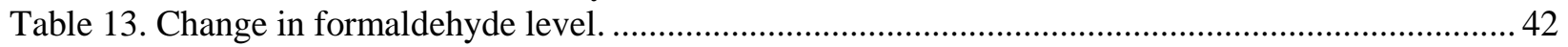

Table 14. Indoor temperature and relative humidity for formaldehyde sub-sample.............................. 44

Table 15. Indoor temperature and temperature setback, by type of thermostat. .................................... 46

Table 16. Model of pre-weatherization indoor temperature (pooled treatment and control groups).......... 47

Table 17. Pre- and post-weatherization evidence of temperature setback, by group and (for treatment homes) whether a new programmable thermostat was installed. ............................49

Table 18. Change in indoor humidity from pre- to post-weatherization.............................................. 52

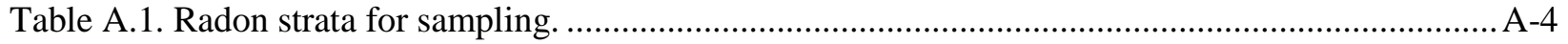

Table A.2. PSU (super-PUMA) allocations to radon strata................................................................. A-5

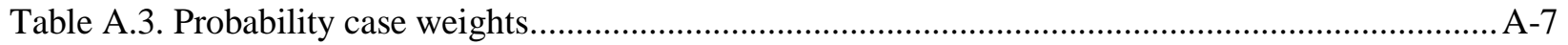

Table A.4. PY08 Population estimates used for post-weighting. ...................................................... A-7

Table B.1 Carbon monoxide issues not noted in many homes. ........................................................ B-6

Table B.2 Sites over 3 ppm CO in pre-Wx period, post-Wx period, or both........................................ B-7

Table B.3 Homes with 5 or more events of 20 ppm of $\mathrm{CO}$ or more................................................ B-8

Table B.4 Homes with 1 or more events of 35 ppm CO or more. ........................................................ B-9

Table B.5 Homes with changes of 1 ppm or more between the pre-Wx and post-Wx periods............. B-10

Table C.1 Radon levels by tenure status and housing value - 245 households in nonmetropolitan counties of New York (Chi and Laquatra 1990)...............................................................

Table C.2 WHO Handbook compares estimated excess lung cancer rates reported from existing

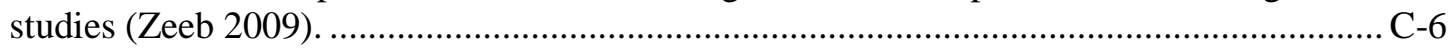

Table C.2 False negative and positive when estimating annual averages based on 1 screening

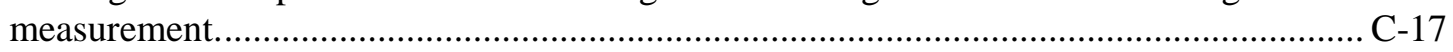

Table C.3. Summary of relevant studies related to radon and weatherization.................................. C-23

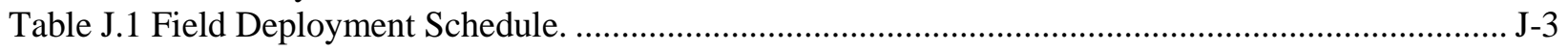





\section{LIST OF ACRONYMS AND ABBREVIATIONS}

$\begin{array}{ll}\text { ACH50 } & \text { Air Changes per Hour at 50 Pascals } \\ \text { ALAA } & \text { Annual Living Area Average Radon Concentration } \\ \text { ASD } & \text { Active Sub-slab Depressurization } \\ \text { ASHRAE } & \text { American Society of Heating, Refrigerating and Air Conditioning Engineers } \\ \text { ASP } & \text { Active Sub-slab Pressurization } \\ \text { BEIR } & \text { Committee on the Biological Effects of Ionizing Radiation } \\ \text { BPI } & \text { Building Performance Institute } \\ \text { CO } & \text { Carbon Monoxide } \\ \text { DOE } & \text { Department of Energy } \\ \text { EASP } & \text { Efficient Active Sub-slab Pressurization Technique } \\ \text { EPA } & \text { Environmental Protection Agency } \\ \text { F } & \text { Fahrenheit Degrees } \\ \text { GSOD } & \text { Global Summary of Day } \\ \text { IARC } & \text { International Agency for Research on Cancer } \\ \text { LBNL } & \text { Lawrence Berkeley National Laboratory } \\ \text { MASC } & \text { Membrane and Short-Circuit Technique } \\ \text { MCS } & \text { Model Conservation Standards } \\ \text { NRRS } & \text { National Residential Radon Survey } \\ \text { NOAA } & \text { National Oceanic and Atmospheric Administration } \\ \text { O2 } & \text { Oxygen } \\ \text { ORD } & \text { Office of Research and Development } \\ \text { ORNL } & \text { Oak Ridge National Laboratory } \\ \text { OSHA } & \text { Occupational Safety and Health Administration } \\ \text { pCi/l } & \text { pico-Curies per liter } \\ \text { ppb } & \text { Parts Per Billion } \\ \text { ppm } & \text { Parts Per Million } \\ \text { PSU } & \text { Primary Sampling Unit } \\ \text { PUMA } & \text { Public Use Microdata Area } \\ \text { PY } & \text { Program Year } \\ \text { WHO } & \text { World Health Organization } \\ \text { WX } & \text { Weatherization }\end{array}$





\title{
ACKNOWLEDGEMENTS
}

The work presented in this report was funded by the U.S. Department of Energy's (DOE) Office of Weatherization and Intergovernmental Programs (OWIP).

This report summarizes the findings from the Indoor Air Quality Study of the Weatherization Assistance Program (WAP) Evaluation. In this field study, the evaluation team completed field study visits with more than $500 \mathrm{WAP}$ clients to directly measure the pre-weatherization and post-weatherization conditions in homes served with the WAP program.

The original design for the study was developed by staff from the Oak Ridge National Laboratory (ORNL) as one component of the National Evaluation of the Weatherization Assistance Program. (National Evaluation of the Weatherization Assistance Program: Preliminary Evaluation Plan for Program 2006 - ORNL/CON-498). As part of the evaluation plan development the design team consulted with and received feedback the Network Planning Committee (41 individuals from the weatherization network).

ORNL contracted with the research team of APPRISE Incorporated, the Energy Center of Wisconsin, Michael Blasnik and Associates, and Dalhoff Associates LLC to conduct the National Evaluation. The Energy Center of Wisconsin took primary responsibility for the design and implementation of this study. Special contributions were made by Paul Francisco (University of Illinois), who prepared the section of the report dealing with indoor humidity and moisture, and conducted the analysis of explanatory factors related to changes in radon levels. In addition, Terry Brennan (Camroden Associates, Inc.) and Beth Hawkins (Oak Ridge National Laboratory) compiled an extensive literature review related to indoor radon, which is included as Appendix $\mathrm{C}$ of this report. Other members of the Evaluation Team who contributed to the design and implementation of this study included:

\author{
The Energy Center of Wisconsin \\ Scott Pigg \\ Ingo Bensch \\ Claire Cowan \\ Charlene Drumm \\ Karen Koski \\ Melanie Lord \\ Andy Mendyk \\ Sarah Payne \\ Nick Sayen \\ John Viner
}

\section{APPRISE Incorporated}

David Carroll

Tim Lenahan

Jim Devlin

Jennifer Frenett

\section{Michael Blasnik and Associates}

Michael Blasnik

Dalhoff Associates LLC

Greg Dalhoff 


\section{Vermont Energy Investment Corporation}

Ken Tohinaka

Matt Sargent

Dick Kornbluth LLC

Dick Kornbluth

The following field staff worked long hours in all kinds of weather to complete the field visits at the appointed time.

\section{Conservation Services Group}

Nick Abreu

Gabe Baldwin

Jonathan Harrison

Margo Labadorf

Franklin Energy

Harrison Fegley

Cole Granrath

Dan Hanson

Tony Hass

Torrance Kramer

Matt McKenney

Scott Schmidt

Richard Stephens

Honeywell

Charles Lewis

Mike Rodgers

KEMA

David Cranford

Mimi Goldberg

Brad Hoover

Eddie Rohilla

Chris Williams

\section{Performance Services Development}

Ed Henneman

Jon Kautz

Joe Kohler

Sean O'Bannion

\section{Southface}

Amelia Godfrey

Benson Johnson

John Kane

\section{Southern Energy Management}

Daniel Connor 
Michael Peaden

Trefoil Home Enterprises

Bill Manning

Independent

Paul Button

Jon McCall

Russ Shaber

Tom Werst

This study could only be completed with the cooperation and contributions of the 88 local weatherization agencies which - in the midst of meeting ambitious production goals - provided us with data, assisted in recruiting households, accompanied study technicians, and arranged for our technicians to borrow equipment when needed. We appreciate the additional burden that our requests place on agency staff and are grateful for all of the assistance that they provided.

The study would not have been possible without the cooperation of the more than 500 households who allowed us to work in their home and helped us by making sure that our metering equipment remained in place and undisturbed.

We received valuable feedback from the following panel of experts who reviewed a preliminary draft of this report:

- H. Gil Peach, H. Gil Peach \& Associates

- Tom Philips, Healthy Building Research

- Brett Singer, Lawrence Berkeley National Laboratory

- Ellen Tohn, Tohn Environmental Strategies

- David Wilson, Oak Ridge National Laboratory

- Jonathan Wilson, National Center for Healthy Housing

We also received many helpful comments on a preliminary draft from staff at the Department of Energy, the Environmental Protection Agency, and the Department of Housing and Urban Development.

Finally, we would like to acknowledge the assistance and guidance of the Oak Ridge National Laboratory, Department of Energy Project Officers, and Department of Energy Headquarters Staff.

Scott Pigg

Dan Cautley 



\section{REPORT SUMMARY}

This report summarizes findings from a national field study of indoor air quality parameters in homes treated under the Weatherization Assistance Program (WAP). The study involved testing and monitoring in 514 single-family homes (including mobile homes) located in 35 states and served by 88 local weatherization agencies.

The study focused on the following five indoor environmental quality parameters:

- Carbon monoxide

- Radon

- Formaldehyde - 7-day tests of formaldehyde concentrations in living-spaces were conducted before and after weatherization on a sub-sample of homes in the study.

- Indoor temperature and humidity - indoor temperature and humidity at the central thermostat was tracked on a 10-minute basis for an average of 26 days preceding and following weatherization.

- Indoor moisture - a visual assessment of above- and below-grade moisture issues was made before and after weatherization.

To account for non-program influences, and isolate the impact of weatherization, approximately a third of the homes (189 homes) were randomly assigned to a control group at the beginning of the study. The control group was geographically matched to the treatment group, and received all of the same testing and monitoring over the same time period as the treatment group homes. However, actual weatherization for the control group was delayed until after completion of the study.

The large majority of the testing and monitoring for the study was implemented during the 2010/11 heating season, with field work taking place in a staggered fashion from early November 2010 through early April 2011. Fieldwork for 23 homes (in three southern states) was implemented between June and August 2011 during the cooling season.

The U.S. Department of Energy (DOE) was particularly interested in determining how weatherization affects radon levels in homes. The study was thus designed to over-sample homes in high radon areas to assure a suitable sample size of homes with measurable pre-existing radon levels and was performed in closed-home conditions where one would expect to capture the greatest potential change related to weatherization. Overall results were then weighted to reflect the general population of single-family homes treated by the program in Program Year (PY) 2008.

The study was not intended to address program policy or health implications related to impacts from the indoor air quality parameters that were addressed.

Key results from the study are as follows:

\section{Carbon Monoxide Production by Combustion Appliances}

Study technicians measured carbon monoxide (CO) production by fuel-fired appliances before and after weatherization. Generally, low incidence rates of actionable $\mathrm{CO}$ were found among combustion appliances in the study (see below). However, given the deadly nature of $\mathrm{CO}$, this does not imply that current combustion-safety practices are unnecessary, or that changes should be made to the program. 
A summary of findings for the three key combustion-safety related appliances in most homes follows:

- Heating systems

- About three quarters of homes have a central, fuel-fired heating system and about 40 percent of these are atmospherically vented, which are of the most concern in terms of combustion safety. Only two of 114 naturally vented systems in the study were found to have inadequate draft.

$\circ$ An incidence rate of $9 \pm 6$ percent was found of natural-draft systems that produced hazardous levels of carbon monoxide (>400ppm) prior to weatherization. Only one system was measured with a hazardous $\mathrm{CO}$ level following weatherization: that reading could not be replicated by the local weatherization agency, and may have been the result of unusual circumstances.

- Water heaters

- Testing for the study revealed a $15 \pm 4$ percent incidence of fuel-fired natural-draft water heaters with inadequate draft (per Building Performance Institute (BPI) guidelines), suggesting that water heaters represent a somewhat greater combustion-spillage risk in homes. Following weatherization, none of the remaining natural-draft water heaters failed a draft test.

- Carbon monoxide production above $400 \mathrm{ppm}$ was found at only about a one in 200 incidence among fuel-fired water heaters in the study.

- Ovens and ranges:

- The study data suggest that prior to weatherization, roughly 10 to 20 percent of fuel-fired ovens (but only about 2 percent of cooktop burners) in homes produce carbon monoxide above the 800 ppm (air-free) level that is the federal standard for new ranges.

- Post-weatherization testing of the same units showed substantial scatter compared to preweatherization results, even among control homes that did not have intervening weatherization activity. This suggests that the simple test procedure used here - and which is also used by many local weatherization agencies - is not particularly reliable for accurately measuring $\mathrm{CO}$ production by ranges and ovens.

\section{Indoor Ambient Carbon Monoxide Levels}

Carbon monoxide data loggers recorded ambient $\mathrm{CO}$ levels in a single central location in the study homes at 1- or 5-minute intervals for about a month before and after weatherization. The data show that:

- Ambient CO levels never exceeded 5 ppm for about two-thirds of homes.

- About one in ten homes had one or more episodes of CO elevation that peaked at $20 \mathrm{ppm}$ or higher prior to weatherization (the highest was $90 \mathrm{ppm}$ ).

- About one in 25 program homes had an indoor $\mathrm{CO}$ level that exceeded $5 \mathrm{ppm}$ for ten percent of the time or more, but only about one in one hundred exceeded this threshold regularly.

These proportions remained substantially unchanged following weatherization for both treated and control homes. A more detailed review of all sites with recorded (persistent or episodic) elevated $\mathrm{CO}$ revealed some cases with likely sources such as an attached garage or operation of a furnace or cook stove; but no clear source for $\mathrm{CO}$ could be identified in other cases. The small number of such cases makes it difficult to draw general conclusions about the impact of weatherization on ambient indoor CO. 


\section{Radon}

Study technicians deployed 7-day, activated-charcoal canisters to measure radon levels in foundation spaces and first-floor living spaces before and after weatherization. These tests were made during the heating season under closed-home conditions. These short-term tests are thus not reflective of expected annual average radon levels in weatherization homes. Key findings for radon follow:

- The study data indicate that the average single-family home in the program has a heating-season indoor radon level of $1.9 \pm 0.1 \mathrm{pCi} / \mathrm{L}$.

- Pre-weatherization radon levels are correlated with pre-weatherization air tightness: tighter homes tend to have higher radon levels.

- The study confirms that elevated radon is relatively rare in mobile homes and in site-built homes in counties identified by EPA as having low radon potential.

- The data from the study suggest that weatherization results in a small, statistically significant (in absolute terms) increase in indoor radon levels. Nationally, the study data suggest an average increase of $0.4 \pm 0.2 \mathrm{pCi} / \mathrm{L}$.

- The impact of weatherization on radon appears to be generally proportional to pre-weatherization levels: homes with low pre-existing radon levels - which constitute the majority of program homes experience only a slight increase in radon levels on average, while homes with pre-existing elevated radon experience a larger average increase following weatherization. On average, the radon impact is thus largest among site-built homes in EPA high-radon-potential counties, and lowest among mobile homes and homes in low-radon potential counties.

- Changes in measured air-leakage rates due to air-sealing efforts — which are intended to reduce air infiltration and yield energy savings were found to be statistically correlated with changes in radon levels in study homes.

- The study provides some evidence that the installation of continuous mechanical ventilation reduces radon levels in homes.

\section{Formaldehyde}

Formaldehyde levels were measured on the first floor above grade for a sub-sample of 131 homes in the study using commercial test badges that were exposed for an average of eight days before and after weatherization. The results indicate the following:

- The average program home has a pre-weatherization indoor formaldehyde concentration of $14 \pm 1$ $\mathrm{ppb}$, which is consistent with formaldehyde levels observed for older homes in other studies. Most homes tested below $30 \mathrm{ppb}$ prior to weatherization.

- Weatherization resulted in a net $1.6 \pm 1.1 \mathrm{ppb}$ increase in formaldehyde levels.

- Formaldehyde levels (and changes in formaldehyde levels) were correlated with indoor humidity (and changes in humidity): higher formaldehyde levels were observed in homes with higher indoor humidity. 
- Mobile homes may have higher formaldehyde levels than site-built homes, and weatherization may have a larger impact on these levels, but the available sample precludes solid conclusions.

\section{Temperature}

Indoor temperature data for the study homes was obtained from data loggers that were hung from the central thermostat for a period that averaged 26 days preceding and following weatherization. The temperature data for homes monitored during the heating season showed that:

- Wintertime indoor temperatures in program homes average $70.3 \pm 0.5 \mathrm{~F}$, but range from less than $60 \mathrm{~F}$ to more than $80 \mathrm{~F}$.

- Households that showed evidence of practicing thermostat setback (based on variation in daily temperature profiles) have indoor temperatures that average $3.0 \pm 0.7 \mathrm{~F}$ lower than households that do not practice setback prior to weatherization.

- One quarter to one third of single-family program homes have a programmable thermostat prior to weatherization: indoor temperatures in these homes average $1.5 \pm 0.5 \mathrm{~F}$ lower than in homes with a manual (or no) thermostat.

- In the month following weatherization, average indoor temperature rose very slightly in the treatment group and fell slightly among the control group households; the net change between the two was an increase of $0.3 \pm 0.2 \mathrm{~F}$.

\section{Humidity and Moisture}

The data loggers that recorded indoor temperature also provided data on humidity levels in the study homes. In addition technicians made a visual inspection for signs of indoor moisture before and after weatherization. The results for the homes monitored during the heating season follow:

- Prior to weatherization, program homes tend to be on the dry side during the heating season: nearly half $(44 \pm 5 \%)$ have wintertime relative humidity below 30 percent, but ten percent or fewer $(6 \pm 4 \%)$ have relative humidity above 50 percent.

- Weatherization was associated with a small but statistically significant $(1.1 \pm 0.6 \%)$ increase in winter relative humidity.

- About $35 \%$ of foundations and $40 \%$ of above-grade spaces had observed moisture problems.

- Water stains were the most common observed moisture problem in both foundations and above-grade spaces. About three in ten above-grade spaces had water stains (31 $\pm 5 \%$ pre-weatherization, $27 \pm 5 \%$ post-weatherization) and about one fifth of foundations had water stains (19 $\pm 5 \%$ pre-weatherization, $24 \pm 5 \%$ post-weatherization). There were no statistically significant changes in observed moisture problems associated with weatherization within the time frame of the study.

- More than half of all pre-weatherization observed water stains and mold were less than two square feet except for foundation water stains, where about half were between two and 32 square feet. 


\section{INTRODUCTION}

\subsection{BACKGROUND}

The U.S. Department of Energy's (DOE's) Weatherization Assistance Program (WAP) has supported energy efficiency improvements to the homes of low-income households in the United States since 1976. The program provides grants, guidance, and other support to state-specific weatherization programs administered by each of the 50 states, the District of Columbia and several U.S. territories. The state programs, in turn, oversee a network of local weatherization agencies-mostly non-profit organizationsthat qualify eligible households, assess their homes' energy efficiency opportunities, install energy-saving measures, and inspect the work. The work performed includes air sealing, insulation upgrades, furnace replacements, and other dwelling-specific measures found to be cost-effective - as well as targeted repairs and home improvements needed to ensure the health and safety for its occupants. The work is done at no cost to eligible participants.

For Program Year (PY) 2008, DOE made available \$279 million in program grants to states for their use in administering their respective statewide programs. These funds were further disseminated to a network of about 900 local weatherization agencies (that are subgrantees under the states) for their use on approximately 100,000 housing units that were weatherized by the program in that year. Many state programs and subgrantees supplement DOE funds with other funding sources for use on the housing units weatherized as part of the DOE program.

Although there have been studies of some state-administered weatherization programs, the overall effectiveness of the national weatherization program has not been formally evaluated since the 1989-90 heating season. The program has evolved significantly since that time, with an increased focus on baseload electric usage, continued evolution of diagnostic tools, new guidelines and best practices for heating-related and health-and-safety measures and adjustments in program rules. More recently, the program has also adjusted to large, temporary funding increases and changes in federal rules spurred by the American Recovery and Reinvestment Act (ARRA).

Consequently, at the direction of the DOE, Oak Ridge National Laboratory (ORNL) is conducting two evaluations of the national weatherization program. The first evaluation - of which this report is a partfocuses on PY2008, which was the last year before substantial ARRA funding became available to the national weatherization network. The second evaluation will focus on the ARRA-funded PY2010.

The purposes of the overall evaluation - and the collection of reports stemming from this effort - are to: (1) provide a comprehensive review of program performance; (2) enable DOE to make any necessary improvements and guide the direction of the program into the next decade; and, (3) provide information of interest to potential funders in order to support leveraging activities. The PY2008 evaluation effort also provides a baseline against which the subsequent ARRA evaluation can be compared.

To guide the overall evaluation of the PY2008 program, ORNL prepared a detailed evaluation plan in 2007 (Ternes et al., 2007). A key element included in that plan was an assessment of non-energy impacts of weatherization, including changes in indoor air quality (IAQ) parameters. Specifically, the ORNL plan called for pre- and post-weatherization field measurements in a national sample of homes to quantify selected IAQ parameters. This report reviews the findings from the field study that ultimately resulted from that vision. 


\subsection{STUDY SCOPE}

The evolution of the scope of the study in terms of time period, geography, housing type and studied parameters is described below.

\subsubsection{Time Period}

The study was implemented under the auspices of the overall retrospective PY2008 evaluation, and the results presented later in this report are weighted to reflect the population of weatherized homes in that year. However, due to the requirement to obtain pre- and post-weatherization data, implementing the study required recruiting homes that were weatherized in PY2010 during the height of the ARRA period. The study thus represents something of a hybrid, reflecting the housing stock, measures and rules and guidelines of PY2010, but weighted to reflect geographic distribution of the program in PY2008. Also, while the network of weatherization providers during the study period is largely the same as PY2008, many of these providers had increased staff and contractors to deal with the increased caseload under ARRA.

\subsubsection{Geography}

Geographically, the study excluded U.S. Territories such as Puerto Rico. Also, for logistical reasons, sampling for the study excluded Alaska and Hawaii. Otherwise, the geographic scope of the study included all lower-48 states and the District of Columbia.

\subsubsection{Housing Type}

The ORNL evaluation plan did not specify the housing types to be included in the study, but the Project Team felt that the project should be focused on single-family housing, which comprised 83 percent of the housing units treated by the program in PY2008. However the study was not constrained as to type of housing within the single-family category: site-built detached and attached homes were included in the scope of the effort, as were manufactured housing (mobile homes), which make up about one in five homes treated by the program. The study also was not restricted by housing tenure: both owner-occupied and rental homes were included in the scope.

\subsubsection{Measurement Parameters}

The 2007 ORNL plan called for making the following pre- and post-weatherization field measurements in 309 weatherization homes and 59 control homes:

- indoor carbon monoxide level;

- indoor and outdoor airborne mold and pollen spores;

- indoor temperature and humidity;

- indoor asbestos level;

- indoor radon level; and,

- refrigerator temperature.

The ORNL scope of work that followed the evaluation plan also recognized that the study presented an opportunity to gather additional data on homes treated by the program, and directed that pre- and postweatherization measurements be made of overall home air leakage, duct leakage and heating system steady-state efficiency. 
At the outset of detailed scoping and budgeting for the project, the Project Team made the case that seasonality issues and the episodic nature of mold and pollen would be difficult to overcome for the relatively short study period. At the same time, the Project Team felt that airborne asbestos impacts related to weatherization would be too transitory to be adequately measured in the relatively few site visits planned for each home. These parameters were therefore dropped from the scope of the study.

On the other hand, as part of a pilot effort to test field instruments and protocols during the 2009/10 heating season, the Project Team demonstrated the feasibility of measuring formaldehyde levels in homes. This parameter was added to the research protocol - though for cost reasons, it was only implemented in a subset of homes.

Moreover, in August 2010, DOE identified radon as a high priority policy issue, and directed ORNL and the Project Team to increase the total sample size for the study to approximately 640 homes. This was done in order to provide an oversample of sites in high-radon areas. However, due to budget constraints on the overall evaluation effort - this target was later reduced to about 540 homes.

The scope of the study was also modified shortly before going into the field to merge it with what had previously been identified as a separate technical study of refrigerator energy use and savings. The field protocol and instruments were therefore modified to include gathering data on refrigerators in the study homes. However, those data (as well as the refrigerator temperature measurements identified above) are not included in the scope of this report.

\subsection{OBJECTIVES}

The primary objectives of the study are as follows:

1. To measure pre-weatherization characteristics of single family homes treated by the program in terms of:
a. carbon monoxide
b. radon
c. formaldehyde
d. temperature
e. humidity and moisture issues

2. To measure weatherization-related changes in the above parameters, controlling for non-program factors.

3. To provide direct pre- and post-weatherization field measurements of air leakage, duct leakage and heating system efficiency for a national probability sample of homes treated by the program.

4. To gather direct field data on refrigerators, refrigerator energy consumption and refrigeration savings from replacement for a national sample of homes treated by the program.

Note that ORNL was not asked to address the program policy or health implications arising from impacts of the program on the selected IAQ parameters. 



\section{APPROACH}

The study employed a randomized control trial design: that is, program-eligible homes were recruited for the study and randomly assigned to either receive weatherization in the middle of the study period or serve as a control group (to account for non-program influences on the parameters of interest). Households in the control group did receive weatherization services, but not until after data collection for the study was complete.

\subsection{SAMPLING}

A national study such as this faces challenging trade-offs between obtaining a sample that is both geographically diverse and logistically feasible. These objectives were met for this study by employing two stages of sampling: first, specific geographic areas were sampled; then weatherization-eligible households were recruited for the study from within sampled geographies.

The geographic sampling used Census units called "super-PUMAs.", The U.S. Census Bureau divides the country into approximately 500 super-PUMAs, each containing between about 400,000 and 900,000 persons. Seventy-five super-PUMAs (in 35 states) were sampled using a sampling approach designed to provide good overall geographic diversity for characterizing the program nationally, as well as to provide adequate sample allocation for characterizing radon levels in homes located in high radon areas. ${ }^{2}$ (The details of the sampling are provided in Appendix A). The sampled geographic areas for the study are shown in Fig. 1. The sample is relatively concentrated in the northern half of the country because statelevel funding for the weatherization program is based on climate: states in cold climates receive more funding for the program than do states in hot climates. The Dakotas, Minnesota, Iowa and parts of Pennsylvania are heavily sampled because these have high radon potential (see Appendix A).

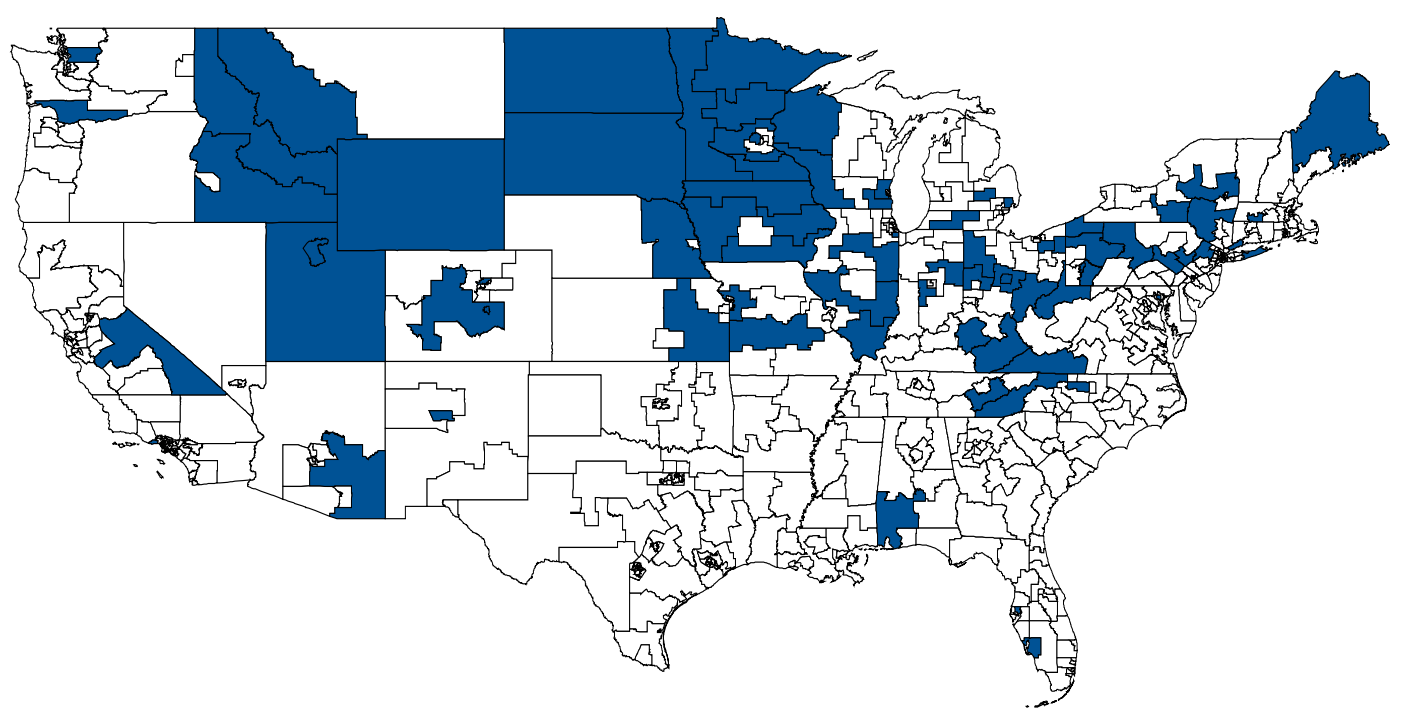

Fig. 1. Sampled geographic areas.

For each sampled geographic area, local weatherization agencies serving the area were contacted to obtain lists of clients in single-family homes that were scheduled to receive weatherization in the near

\footnotetext{
${ }^{1}$ PUMA stands for Public-Use Microdata Area. "Super-PUMA" refers to larger geographic areas that are aggregations of smaller PUMAs.

${ }^{2}$ Data for 71 of the sampled 75 super-PUMAs are incorporated in this report: data for the remaining four super-PUMAs (in hot climates) was gathered during the summer of 2011 and was not available at the time this report was written.
} 
future. Weatherization agencies were not formally sampled, but an attempt was made to prioritize contact with agencies whose service territory comprised a larger fraction of the sampled area. Each contacted agency was asked to provide a list of up to 24 single-family homes that had not yet received weatherization, but for which weatherization could be completed within the constraints of the study. ${ }^{3}$ In most areas, a single weatherization agency was able to provide a sufficient list of candidate homes; overall, the homes in the study were served by 88 local agencies.

Lists of candidate homes were randomly assigned to treatment and control groups, and then households were contacted directly for recruitment into the study. Recruiting targets for each sampled area ranged from six to eight homes, with the sample for each area targeted to comprise 60 percent treatment-group sites and 40 percent control-group sites. ${ }^{4}$ Households in the treatment group received total cash incentives of $\$ 120$ for participation in the study; control-group households received $\$ 170$ (the higher incentive was to compensate for the delay in weatherization). The script for recruiting households is included in Appendix I.

A total of 536 homes were recruited for the study and received at least one site visit. However, 22 homes were later removed: 15 of these were not weatherized within the overall time frame of the study, four had issues with clients that moved or did not respond to requests for subsequent site visits, and three were determined to be multifamily properties and therefore not within the scope of the study. The final analysis data set therefore comprises 514 households, of which $325(63 \%)$ were assigned to the treatment group and 189 (37\%) were assigned to the control group for which weatherization was delayed until completion of fieldwork for the study.

\subsection{DATA COLLECTION}

Fieldwork for the study consisted of four site visits to each home:

Pre-weatherization

Visit 1 (approximately one month prior to the start of weatherization work)

- deployment of temperature and relative humidity data loggers

- deployment of carbon monoxide data loggers

- deployment of pre-weatherization radon and formaldehyde test samplers

- detailed pre-weatherization site data gathering and testing

Visit 2 (one week after Visit 1)

- retrieval of pre-weatherization radon and formaldehyde samplers

Post-weatherization

Visit 3(approximately three weeks following the completion of weatherization work)

- deployment of post-weatherization radon and formaldehyde test samplers

- detailed post-weatherization site data gathering and testing

Visit 4 (one week after Visit 3)

- retrieval of post-weatherization radon and formaldehyde samplers

- retrieval of data loggers deployed at Visit 1

Local weatherization agencies were provided with a target three- to four-week time period to complete all weatherization work on the treatment homes. The period was intended to allow for collection of three to

\footnotetext{
${ }^{3}$ Agencies with more than 24 candidate homes meeting the study criteria were asked to provide a list of the 24 most-recently audited homes.

${ }^{4}$ A total of 97 households were switched from one group to another in order to meet the recruitment quotas for treatment and control sites in a given area. These switches were made prior to any contact with the households.
} 
four weeks of pre- and post-weatherization monitoring data on carbon dioxide, temperature and humidity. Weatherization was completed within this requested time period for 80 percent of the treatment group-and within a week of the time period for 90 percent - but work was delayed for some sites for a variety of reasons. In some cases, it was possible to delay return visits to the sites, but a few sites had to be dropped from the study because weatherization could not be completed in time.

Experienced residential testing technicians were deployed for Visits 1 and 3, which (as described below) involved a significant amount of testing and data collection regarding the house, mechanical systems and appliances and air leakage characteristics. Prior to field deployment, a two-day training was conducted with field technicians to cover all aspects of the testing protocol and provide an opportunity to implement the protocol in practice homes. In addition, a member of the Project Team monitored each technician during his/her first week in the field. The fieldwork was also preceded (during the 2009/2010 heating season) by a pilot project involving eight homes designed to test and refine the monitoring, testing and data collection protocols for the study. (Appendix D provides more details on the training and field protocol.)

The fieldwork was staged in seven rounds, starting in early November 2010. The first six rounds (491 homes) were completed within the 2010/11 heating season. The seventh round, which involved 23 homes in three southern states, ran from mid-June through mid-August 2011. Appendix J provides a more detailed schedule of the fieldwork.

Actual weatherization of homes in the control group was delayed until the completion of fieldwork for the study. For analysis purposes, these homes were assigned pseudo weatherization work dates that corresponded to the weatherization window for treatment-group homes in the same geographic area.

\subsubsection{Site Data}

As noted above, the first and third site visits to the home (corresponding to the pre- and postweatherization periods) involved detailed data collection and measurements on the home, mechanical systems and appliances. The complete data collection instrument is included in Appendix E, and summarized as follows:

1. General characteristics of the home (e.g., type of structure, square footage, foundation types)

2. Thermostat type and settings

3. Evidence of moisture (above and below-grade);

4. Heating system characteristics, draft and combustion analysis

5. Water heater characteristics, draft and $\mathrm{CO}$ production

6. Range, oven and other unvented device characteristics and $\mathrm{CO}$ production

7. Air leakage and zone pressure diagnostics

8. Duct leakage and air-handler pressurization measurements

As noted previously, the site protocol also included gathering information on-and meteringrefrigerators for a separate technical study. Those data are not within the scope of this report, however.

\subsubsection{Testing and Monitoring}

In addition to the data gathered directly by the field technicians, testing and monitoring for key IAQ parameters was implemented at each site. These are discussed in more detail in the Findings section for each parameter, but summarized here. 


\section{Radon and Formaldehyde}

Seven-day radon and formaldehyde tests were implemented between Visits 1 and 2 (pre-weatherization) and Visits 3 and 4 (post-weatherization). Radon levels were measured in basements and crawlspaces as well as in a main living space on the first floor above grade. For budget reasons, formaldehyde testing was limited to one treatment-group home and one control-group home in each sampled geographic area and was measured in the same location as the first-floor radon test. The on-site protocol called for a detailed description (and photos) of the location of the deployed samplers so that the post-weatherization sampling location would match the pre-weatherization location.

\section{Carbon Monoxide}

A carbon monoxide data logger was deployed at each site (in the same location as the first-floor radon and formaldehyde samplers), and recorded a snapshot of indoor ambient $\mathrm{CO}$ either every minute or every five-minutes (two different types of loggers with different recording capabilities were used). In addition to direct monitoring of indoor $\mathrm{CO}$ levels, thermocouple data loggers were deployed to track the operation of some ovens and unvented space heaters at some sites as an aid to interpreting the indoor ambient $\mathrm{CO}$ data.

\section{Temperature and Humidity}

A data logger was hung from the primary thermostat at each site during Visit 1 for tracking indoor temperature and relative humidity. The data logger recorded a snapshot of indoor conditions every 10 minutes throughout the pre- and post-weatherization study periods.

\subsubsection{Occupant Survey}

All participants in the study were administered an extensive survey covering a number of topics, including basic demographics, indoor comfort and health issues. The questions for this survey were drawn from a larger survey instrument that is being implemented in a general sample of program households under a separate task in the PY2008 evaluation effort. The version of the instrument that was used for this study is included in Appendix G.

The original intent was to implement the survey - which contains numerous questions related to comfort and health conditions in the preceding 12 months-prior to weatherization work occurring in the study homes, but delays in obtaining federal approval for the survey resulted in the survey being implemented shortly after weatherization in about half the cases. The survey was implemented by telephone, and had a response rate of 98 percent among the study participants.

\subsubsection{Weatherization Measures}

The local weatherization agencies that provided the candidate homes were not instructed to follow any special protocols for the work on the homes in the study: they were to weatherize the homes as they would normally. However, they were asked to provide detailed information about the measures installed - and diagnostic tests performed - in the treatment group homes. These data allowed comparison to similar data gathered for a much larger national sample of PY2008 homes treated by the program.

\subsubsection{Weather Data}

Outdoor conditions were particularly relevant to the analysis of indoor temperature and humidity. Study sites were matched to nearby NOAA weather stations by geocoding the site address, then implementing a 
matching algorithm that looked for weather stations that were both close and (for mountainous areas) at a comparable altitude as the site. ${ }^{5}$ Ninety percent of the sites were matched to a weather station that was within 40 miles, and none exceeded 70 miles. All weather stations were within 1,000 feet of the site elevation, and 90 percent were within 350 feet. The weather data consisted of daily temperature, dewpoint temperature and air pressure readings from the stations.

\footnotetext{
${ }^{5}$ Station information and weather data were obtained from: http://www7.ncdc.noaa.gov/CDO/cdoselect.cmd?datasetabbv=GSOD\&resolution=40
} 



\section{CHARACTERISTICS OF STUDY HOMES}

Tables 1,2, and 3 on the following pages summarize some key attributes of the homes and households in the study, as well as the weatherization work that was performed. As a point of comparison, statistics (where available) are shown from a much larger national sample $(n=13,085)$ of housing units treated by the program in PY08 that were collected as part of the larger evaluation effort. The study households are shown as a pooled sample - which combines the treatment and control groups - and also broken out into the separate treatment and control groups. In the sections that follow, statistics are reported for the pooled sample as a best estimate of the pre-weatherization characteristics of homes in the program.

The breakouts for the pooled sample show that three in four single-family homes in the program are sitebuilt, detached structures and about one in five is a mobile home. ${ }^{6}$ A small proportion of homes are single-family, attached housing units. ${ }^{7}$ A majority of the homes in the study are single-story structures with basements and heated by fuel-fired, forced air furnaces. About a third of the study homes are occupied by all-senior households, but more than a third also have a child in the home.

The treatment and control groups are very similar in most respects, and largely mirror the characteristics of the PY08 population. The main exception-and it is an important one-is the mix of measures and spending on weatherization: the study homes have a higher incidence of measures and show higher weatherization costs than occurred in PY08, no doubt due to the influx of ARRA funding for the program in PY10, when the study was implemented.

Table 1. Selected home characteristics, study sample (weighted) and WAP population.

\begin{tabular}{|c|c|c|c|c|}
\hline & $\begin{array}{c}\text { Pooled groups } \\
(n=514)\end{array}$ & $\begin{array}{c}\text { Treatment group } \\
(\mathbf{n}=\mathbf{3 2 5})\end{array}$ & $\begin{array}{c}\text { Control group } \\
(n=189)\end{array}$ & $\begin{array}{c}\text { PY08 SF } \\
\text { population }\end{array}$ \\
\hline \multicolumn{5}{|l|}{ Housing type } \\
\hline Site-built, detached & $71 \%-$ & $70 \%-$ & $72 \%-$ & $70 \%$ \\
\hline Site-built, attached & $7 \%-$ & $8 \%-$ & $6 \%-$ & $6 \%$ \\
\hline Mobile home & $22 \%-$ & $22 \%-$ & $22 \%-$ & $24 \%$ \\
\hline \multicolumn{5}{|l|}{$\begin{array}{l}\text { Location of home (classified by } \\
\text { occupant) }\end{array}$} \\
\hline City & $33 \% \pm 6$ & $32 \% \pm 6$ & $35 \% \pm 8$ & \\
\hline Town & $26 \% \pm 4$ & $28 \% \pm 6$ & $23 \% \pm 5$ & \\
\hline Suburbs & $10 \% \pm 5$ & $11 \% \pm 5$ & $9 \% \pm 5$ & \\
\hline Rural & $31 \% \pm 4$ & $29 \% \pm 6$ & $33 \% \pm 7$ & \\
\hline \multicolumn{5}{|l|}{ Stories } \\
\hline 1 & $66 \% \pm 5$ & $63 \% \pm 6$ & $69 \% \pm 6$ & $61 \% \pm 2$ \\
\hline 1.5 & $10 \% \pm 3$ & $11 \% \pm 4$ & $8 \% \pm 4$ & \\
\hline 2 & $21 \% \pm 5$ & $21 \% \pm 6$ & $19 \% \pm 6$ & $37 \% \pm 2$ \\
\hline$>2$ & $2 \% \pm 1$ & $2 \% \pm 1$ & $2 \% \pm 2$ & $2 \% \pm 1$ \\
\hline Split-level & $2 \% \pm 1$ & $3 \% \pm 2$ & $2 \% \pm 2$ & - \\
\hline
\end{tabular}

\footnotetext{
${ }^{6}$ The weighted proportion of mobile homes is 22 percent in all cases, because the sample was post-stratified and weighted to reflect this proportion, based on DOE tracking data for all single-family homes treated by the program in PY2008. See Appendix A.

${ }^{7}$ The distinction between single-family, attached housing and multifamily housing can be a fine one. The PY2008 evaluation effort defines single-family, detached housing as housing that shares at least one wall (extending from the basement to the attic) with another housing unit, but does not have any other housing unit above or below, and does not share heating or water heating equipment. Row and townhouses are thus generally classified as single-family attached housing. A side-by-side duplex would also be classified as single-family, attached, if it does not share a basement or mechanical systems. An up-and-down duplex, however, would be considered small multifamily housing.
} 
Table 1. (continued)

\begin{tabular}{|c|c|c|c|c|}
\hline & $\begin{array}{c}\text { Pooled groups } \\
(n=514)\end{array}$ & $\begin{array}{c}\text { Treatment group } \\
(\mathbf{n}=325)\end{array}$ & $\begin{array}{c}\text { Control group } \\
(n=189)\end{array}$ & $\begin{array}{c}\text { PY08 SF } \\
\text { population }\end{array}$ \\
\hline \multicolumn{5}{|l|}{ Attached garage? } \\
\hline Yes & $76 \% \pm 5$ & $77 \% \pm 7$ & $76 \% \pm 7$ & - \\
\hline No & $24 \% \pm 5$ & $23 \% \pm 8$ & $24 \% \pm 7$ & - \\
\hline \multicolumn{5}{|l|}{ Dominant foundation type $e^{\dagger \dagger}$} \\
\hline Basement & $45 \% \pm 6$ & $45 \% \pm 7$ & $44 \% \pm 6$ & - \\
\hline Crawlspace & $23 \% \pm 6$ & $23 \% \pm 7$ & $23 \% \pm 5$ & - \\
\hline Pier & $23 \% \pm 2$ & $24 \% \pm 1$ & $22 \%--$ & - \\
\hline Slab & $6 \% \pm 2$ & $5 \% \pm 2$ & $7 \% \pm 4$ & - \\
\hline Space over garage & $1 \% \pm 1$ & $1 \% \pm 1$ & $1 \% \pm 2$ & - \\
\hline Mixed & $2 \% \pm 1$ & $2 \% \pm 1$ & $3 \% \pm 1$ & - \\
\hline \multicolumn{5}{|l|}{ Mean floor area $\left(\mathbf{f t}^{2}\right)$} \\
\hline Including basements & $1,710 \pm 80$ & $1,740 \pm 80$ & $1,630 \pm 80$ & - \\
\hline Excluding basements & $1,290 \pm 50$ & $1,310 \pm 60$ & $1,240 \pm 60$ & - \\
\hline \multicolumn{5}{|l|}{$\begin{array}{l}\text { Mean volume } \\
\left(\text { nearest } 100 \mathbf{f t}^{3}\right)\end{array}$} \\
\hline Including basements & $13,200 \pm 600$ & $13,400 \pm 700$ & $12,800 \pm 700$ & - \\
\hline Excluding basements & $10,200 \pm 400$ & $10,300 \pm 500$ & $9,900 \pm 500$ & - \\
\hline \multicolumn{5}{|l|}{$\begin{array}{l}\text { Mean pre-weatherization } \\
\text { air leakage }(\mathrm{ACH50})^{*}\end{array}$} \\
\hline Including basements & $16.2 \pm 1.4$ & $16.2 \pm 1.3$ & $16.2 \pm 1.7$ & - \\
\hline Excluding basements & $19.8 \pm 1.7$ & $19.9 \pm 1.6$ & $19.8 \pm 2.0$ & - \\
\hline \multicolumn{5}{|l|}{ Heating system fuel } \\
\hline Natural gas & $55 \% \pm 7$ & $54 \% \pm 8$ & $55 \% \pm 8$ & $54 \% \pm 2$ \\
\hline Propane & $10 \% \pm 3$ & $9 \% \pm 3$ & $11 \% \pm 5$ & $11 \% \pm 1$ \\
\hline Fuel oil & $17 \% \pm 6$ & $18 \% \pm 6$ & $16 \% \pm 6$ & $12 \% \pm 1$ \\
\hline Electricity & $16 \% \pm 4$ & $17 \% \pm 5$ & $14 \% \pm 5$ & $19 \% \pm 1$ \\
\hline Other & $3 \% \pm 2$ & $2 \% \pm 1$ & $3 \% \pm 3$ & $3 \% \pm 1$ \\
\hline \multicolumn{5}{|l|}{ Heating system type } \\
\hline Forced-air & $75 \% \pm 6$ & $72 \% \quad \pm 7$ & $80 \% \pm 6$ & $72 \% \pm 1$ \\
\hline Boiler & $12 \% \pm 5$ & $13 \% \pm 7$ & $8 \% \pm 4$ & $9 \% \pm 1$ \\
\hline Other & $13 \% \pm 4$ & $14 \% \pm 4$ & $12 \% \pm 5$ & $19 \% \pm 1$ \\
\hline \multicolumn{5}{|l|}{ Water heater fuel } \\
\hline Natural gas & $45 \% \pm 6$ & $46 \% \pm 7$ & $42 \% \pm 8$ & $48 \% \pm 2$ \\
\hline Propane & $4 \% \pm 2$ & $2 \% \pm 1 \%$ & $9 \% \pm 4$ & $7 \% \pm 1$ \\
\hline Electric & $46 \% \pm 5$ & $45 \% \pm 5$ & $46 \% \pm 6$ & $42 \% \pm 2$ \\
\hline Other & $5 \% \pm 4$ & $7 \% \pm 5$ & $2 \% \pm 2$ & $3 \% \pm 1$ \\
\hline \multicolumn{5}{|l|}{ Range/oven fuel } \\
\hline Natural gas or propane & $43 \% \pm 7$ & $44 \% \pm 6$ & $41 \% \pm 9$ & - \\
\hline Electric & $57 \% \pm 6$ & $56 \% \pm 6$ & $59 \% \pm 8$ & - \\
\hline
\end{tabular}

'Estimated from other (DF-2) evaluation data for single-family homes (site-built and manufactured). Excludes AK and HI.

${ }^{\dagger}$ Dominant foundation type here means any foundation type making up more than 50 percent of the total foundation footprint. ( \pm values are approximate $90 \%$ confidence intervals)

* Air Changes per Hour at 50 Pascals 
Table 2. Selected demographic characteristics, study sample (weighted) and WAP population.

\begin{tabular}{|c|c|c|c|c|}
\hline & $\begin{array}{l}\text { Pooled groups } \\
\quad(n=514)\end{array}$ & $\begin{array}{l}\text { Treatment group } \\
(n=325)\end{array}$ & $\begin{array}{c}\text { Control group } \\
(n=189)\end{array}$ & $\begin{array}{c}\text { PY08 SF } \\
\text { population }^{\dagger}\end{array}$ \\
\hline \multicolumn{5}{|l|}{ Number of household members } \\
\hline 1 & $35 \% \pm 4$ & $33 \% \pm 6$ & $40 \% \pm 6$ & $40 \% \pm 1$ \\
\hline 2 & $25 \% \pm 4$ & $24 \% \pm 4$ & $26 \% \pm 7$ & $23 \% \pm 1$ \\
\hline 3 & $13 \% \pm 3$ & $16 \% \pm 4$ & $8 \% \pm 3$ & $14 \% \pm 1$ \\
\hline 4 & $13 \% \pm 3$ & $14 \% \pm 4$ & $10 \% \pm 4$ & $11 \% \pm 1$ \\
\hline $5+$ & $14 \% \pm 3$ & $13 \% \pm 3$ & $15 \% \pm 5$ & $12 \% \pm 1$ \\
\hline \multicolumn{5}{|l|}{$\%$ of households with } \\
\hline Child in home & $37 \% \pm 3$ & $38 \% \pm 4$ & $34 \% \pm 6$ & $33 \% \pm 1$ \\
\hline Senior $(60+)$ in home & $48 \% \pm 5$ & $47 \% \pm 5$ & $50 \% \pm 6$ & $50 \% \pm 1$ \\
\hline All seniors $(60+)$ & $32 \% \pm 4$ & $31 \% \pm 5$ & $35 \% \pm 5$ & \\
\hline \multicolumn{5}{|l|}{$\begin{array}{l}\text { Respondent race } \\
\text { (multiple responses allowed) }\end{array}$} \\
\hline White & $76 \% \pm 7$ & $78 \% \pm 6$ & $74 \% \pm 7$ & $69 \% \pm 3$ \\
\hline Black/African American & $16 \% \pm 6$ & $15 \% \pm 6$ & $19 \% \pm 6$ & $22 \% \pm 3$ \\
\hline Other & $10 \% \pm 3$ & $10 \% \pm 4$ & $9 \% \pm 4$ & $3 \% \pm 1$ \\
\hline Hispanic? & $10 \% \pm 4$ & $10 \% \pm 4$ & $11 \% \pm 4$ & $7 \% \pm 1$ \\
\hline $\begin{array}{l}\text { Years lived in current home } \\
\text { (mean) }\end{array}$ & $15 \pm 1$ & $15 \pm 1$ & $16 \pm 3$ & \\
\hline $\begin{array}{l}\text { \% of households where someone } \\
\text { is typically home on a weekday }\end{array}$ & $86 \% \pm 3$ & $85 \% \pm 4$ & $89 \% \pm 5$ & - \\
\hline Tenure & & & & \\
\hline Own & $92 \% \pm 2$ & $93 \% \pm 2$ & $91 \% \pm 4$ & $88 \% \pm 1$ \\
\hline Rent & $8 \% \pm 2$ & $7 \% \pm 2$ & $8 \% \pm 4$ & $11 \% \pm 1$ \\
\hline Other & $<1 \%$ & $0 \%$ & $1 \% \pm 1$ & $<1 \%$ \\
\hline \multicolumn{5}{|l|}{ Smoking } \\
\hline \multicolumn{5}{|l|}{ Rules in home } \\
\hline Not allowed & $71 \% \pm 5$ & $73 \% \pm 6$ & $67 \% \pm 7$ & - \\
\hline Some times/places & $14 \% \pm 3$ & $13 \% \pm 4$ & $17 \% \pm 5$ & - \\
\hline Permitted & $15 \% \pm 3$ & $14 \% \pm 4$ & $16 \% \pm 5$ & - \\
\hline \multicolumn{5}{|l|}{ Respondent smokes... } \\
\hline ...everyday & $23 \% \pm 3$ & $20 \% \pm 5$ & $27 \% \pm 7$ & - \\
\hline ...some days & $5 \% \pm 2$ & $6 \% \pm 3$ & $4 \% \pm 3$ & - \\
\hline ...not at all & $72 \% \pm 4$ & $74 \% \pm 6$ & $69 \% \pm 7$ & - \\
\hline \multicolumn{5}{|l|}{$\begin{array}{l}\text { Respondent's highest level of } \\
\text { education }\end{array}$} \\
\hline None & $2 \% \pm 1$ & $2 \% \pm 1$ & $2 \% \pm 2$ & - \\
\hline $\mathrm{K}-12$, no degree & $20 \% \pm 4$ & $20 \% \pm 5$ & $22 \% \pm 6$ & - \\
\hline High school diploma & $35 \% \pm 4$ & $35 \% \quad \pm 4$ & $34 \% \pm 7$ & - \\
\hline Some college, no degree & $21 \% \pm 3$ & $20 \% \pm 4$ & $23 \% \pm 7$ & - \\
\hline Associate's degree & $10 \% \pm 3$ & $10 \% \pm 3$ & $9 \% \pm 4$ & - \\
\hline Bachelor's degree & $11 \% \pm 3$ & $12 \% \pm 3$ & $8 \% \pm 4$ & - \\
\hline Master's degree & $2 \% \pm 1$ & $1 \% \pm 1$ & $2 \% \pm 3$ & - \\
\hline Professional degree & $<1 \%$ & $0 \%$ & $<1 \%$ & - \\
\hline Doctorate degree & $<1 \%$ & $0 \%$ & $1 \% \pm 1$ & - \\
\hline
\end{tabular}

${ }^{\dagger}$ Estimated from other (DF-2) evaluation data for single-family homes (site-built and manufactured). Excludes AK and HI. ( \pm values are approximate $90 \%$ confidence intervals) 
Table 3. Selected weatherization characteristic, study sample (weighted) and WAP population.

\begin{tabular}{|c|c|c|c|c|}
\hline & $\begin{array}{l}\text { Pooled groups } \\
\qquad(\mathrm{n}=\mathbf{5 1 4})\end{array}$ & $\begin{array}{c}\text { Treatment } \\
\text { group } \\
(\mathbf{n}=325)\end{array}$ & $\begin{array}{l}\text { Control group } \\
\quad(n=189)\end{array}$ & $\begin{array}{c}\text { PY08 SF } \\
\text { population }\end{array}$ \\
\hline \multicolumn{5}{|l|}{ Air Leakage (CFM50) } \\
\hline Pre-weatherization & $3,227 \pm 267$ & $3,306 \pm 316$ & $3,096 \pm 356$ & $3,533 \pm 61$ \\
\hline Post-weatherization & $2,200 \pm 149$ & $2,291 \pm 180$ & & $2,314 \pm 45$ \\
\hline \multicolumn{5}{|l|}{ Insulation } \\
\hline Attic & $67 \% \pm 7$ & $67 \% \pm 7$ & & $58 \% \pm 2$ \\
\hline Wall & $19 \% \pm 6$ & $19 \% \pm 6$ & & $7 \% \pm 1$ \\
\hline Other & $47 \% \pm 8$ & $47 \% \quad \pm 7$ & & $23 \% \pm 1$ \\
\hline \multicolumn{5}{|l|}{ Heating system replacement } \\
\hline For energy savings & $19 \% \pm 5$ & $19 \% \pm 5$ & & $12 \% \pm 1$ \\
\hline For health and safety & $9 \% \pm 4$ & $9 \% \pm 4$ & & $12 \% \pm 1$ \\
\hline \multicolumn{5}{|l|}{ Water heater replacement } \\
\hline For energy savings & $6 \% \pm 3$ & $6 \% \pm 3$ & & $3 \% \pm 1$ \\
\hline For health and safety & $3 \% \pm 2$ & $3 \% \pm 2$ & & $6 \% \pm 1$ \\
\hline \multicolumn{4}{|l|}{ Ventilation } & $10 \% \pm 1$ \\
\hline Exhaust fan & $29 \% \pm 8$ & $29 \% \pm 8$ & & $13 \% \pm 1$ \\
\hline Whole house ventilation & $2 \% \pm 2$ & $2 \% \pm 2$ & & $<1 \%$ \\
\hline \multicolumn{5}{|l|}{ Health \& safety } \\
\hline $\mathrm{CO}$ detector & $70 \% \pm 9$ & $70 \% \pm 9$ & & $56 \% \pm 2$ \\
\hline Smoke alarm & $50 \% \pm 10$ & $50 \% \pm 10$ & & $46 \% \pm 2$ \\
\hline $\begin{array}{r}\text { Mean weatherization cost } \\
\text { (All funding sources) }\end{array}$ & $\$ 4,360 \pm 430$ & $\$ 4,580 \pm 490$ & & $\$ 3,300 \pm 100$ \\
\hline
\end{tabular}

${ }^{\dagger}$ Estimated from other (DF-2) evaluation data for single-family homes (site-built and manufactured). Excludes AK and HI.

( \pm values are approximate $90 \%$ confidence intervals) 


\section{FINDINGS}

In this section results for the five key IAQ parameters for the study are presented:

- Carbon monoxide

- Radon

- Formaldehyde

- Temperature

- Humidity

For each, the treatment and control groups are pooled to describe pre-weatherization conditions in program homes, and then changes associated with weatherization are looked at separately across the groups.

\subsection{CARBON MONOXIDE}

Carbon monoxide (CO) is a colorless, odorless, and poisonous gas. Its toxicity stems from the fact that it readily binds with hemoglobin in blood, which reduces the amount of oxygen available to organs. Carbon monoxide in buildings is most commonly associated with incomplete combustion. Potential sources including unvented combustion appliances (including space heaters and kitchen ranges), vented combustion appliances (furnaces, boilers, water heaters, free-standing stoves, fireplaces etc.) under conditions in which some of the combustion products "spill" (are released inside the building), gasoline engines (including automobiles started or operated in attached garages, and engine-generator sets operated indoors), charcoal grills operated indoors, and smoking. ${ }^{8}$

Carbon monoxide was measured in study homes using several methods. Where primary space heating and/or water heating appliances were vented atmospherically and included a draft hood or barometric damper (features that can allow significant spillage of combustion products into a home), field technicians performed a worst case depressurization test and observed any spillage. ${ }^{9}$ Technicians measured carbon monoxide concentration in appliance vent systems either at the conclusion of the worst case depressurization test or later during a steady state efficiency test, and in room air near the appliances during this testing. In the case of gas cookstoves, carbon monoxide and oxygen concentrations were measured above each operating stove top burner, and in the oven vent. Where unvented space heaters were found, technicians measured oxygen and $\mathrm{CO}$ in or just beyond the vent.

Because CO spillage in the home represents an immediate safety hazard, the study protocol called for notifying occupants (and the local weatherization agencies) whenever measured CO produced by an appliance exceeded $100 \mathrm{ppm}$.

In addition to this on-site testing of $\mathrm{CO}$ production by appliances, a carbon monoxide monitoring device was placed in each home. These monitors were placed on the lowest main living level, almost always near the radon canister. One of two different models of monitoring device was used in each home, with one recording $\mathrm{CO}$ at one-minute intervals, the other at five-minute intervals. ${ }^{10}$ Both types included an alarm, set to trigger at a CO concentration of $200 \mathrm{ppm}$. The logged data indicate that there were no instances in which this alarm was triggered during the course of the study.

\footnotetext{
${ }^{8}$ See http://www.cpsc.gov/cpscpub/pubs/466.html.

${ }^{9}$ Under this protocol, vent fans, doors, and blowers are operated so as to create the most negative possible pressure in the appliance area.

${ }^{10}$ The CO loggers employed were Lascar USB-EL300 (five-minute) and BW GasAlert Extreme CO loggers (one-minute).
} 


\subsubsection{Heating Systems}

Most fuel fired appliances used for residential space heating and water heating include a venting system to remove combustion products from the home. Failure of venting systems, i.e. the spillage of combustion products into the home, can represent a health and safety hazard, in particular if significant levels of carbon monoxide are present in the combustion gasses. Many (but not all) common vented combustion appliances depend on the natural buoyancy of warm combustion products for proper venting operation. Heating systems with a draft hood (or barometric damper, typical of oil-fired systems) are classified here as natural-draft appliances. This category excludes induced-draft appliances, which - though relying on natural buoyancy for proper venting - lack draft diverters, and are less susceptible to spillage. The focus here is on combustion products spillage among appliances with draft diverters and barometric dampers.

One commonly used metric of proper venting operation is draft pressure, the pressure induced in the vent system when low-density combustion products flow up the vent. (Draft pressure is measured in the vent relative to the room air around the appliance, and thus generally reported as a negative value.) Draft pressure in any building depends on chimney height and diameter, heating system sizing, and other factors, and is also variable with operating conditions including outdoor temperature. Building Performance Institute (BPI), for example, suggests acceptable draft pressures ranging from -0.5 Pa to -2.5 $\mathrm{Pa}$ as a function of temperature (BPI, 2012). The BPI criteria are used here as a cutoff for identifying systems with low draft pressure.

On a weighted basis, the full study sample comprised 72 percent central, fuel-fired primary heating systems, 20 percent electric systems and 8 percent non-central (or no) heating systems. The preweatherization venting types for the central, fuel-fired systems were as follows:

- 38 percent atmospheric

- 39 percent induced-draft

- 23 percent power-vented

The focus here is on the atmospheric systems that present the greatest risk for spillage.

Figure 2 shows the distribution of measured draft pressure for the 114 sites with naturally vented heating systems and draft pressure test results. ${ }^{11}$ All but two of the systems passed the BPI criteria for acceptable draft. No significant relationship was observed between draft pressure and outdoor temperature or number of stories.

In terms of CO production, the BPI protocol calls for immediate action when CO levels are in the range of $100-400 \mathrm{ppm}$ and the appliance fails a draft test, as well as when CO levels are above 400 ppm regardless of system draft. Among the 111 tested systems in the study sample, seven systems tested at more than 400 ppm; these ranged from just over $400 \mathrm{ppm}$ to more than 2,600 ppm of CO (Fig. 3). When two additional systems that were replaced on an emergency basis (due to high $\mathrm{CO}$ measured by the local weatherization agency) prior to the initial site visit are included, this suggests a weighted incidence of high $\mathrm{CO}$ among program homes with atmospheric heating systems is estimated at $10 \pm 5$ percent.

Weatherization resulted in just under half of the naturally vented systems being replaced (including six of the seven treatment-group sites with measured $\mathrm{CO}$ exceeding $400 \mathrm{ppm}$ prior to weatherization), with 75 percent of the replacements for energy savings, 17 percent for health \& safety reasons and 8 percent for reasons that could not be determined from the data provided by the local weatherization agency.

\footnotetext{
${ }^{11}$ The draft-test results presented here omit four sites that received heating system service work prior to the initial study site visit, as well as three systems that were replaced prior to the study.
} 


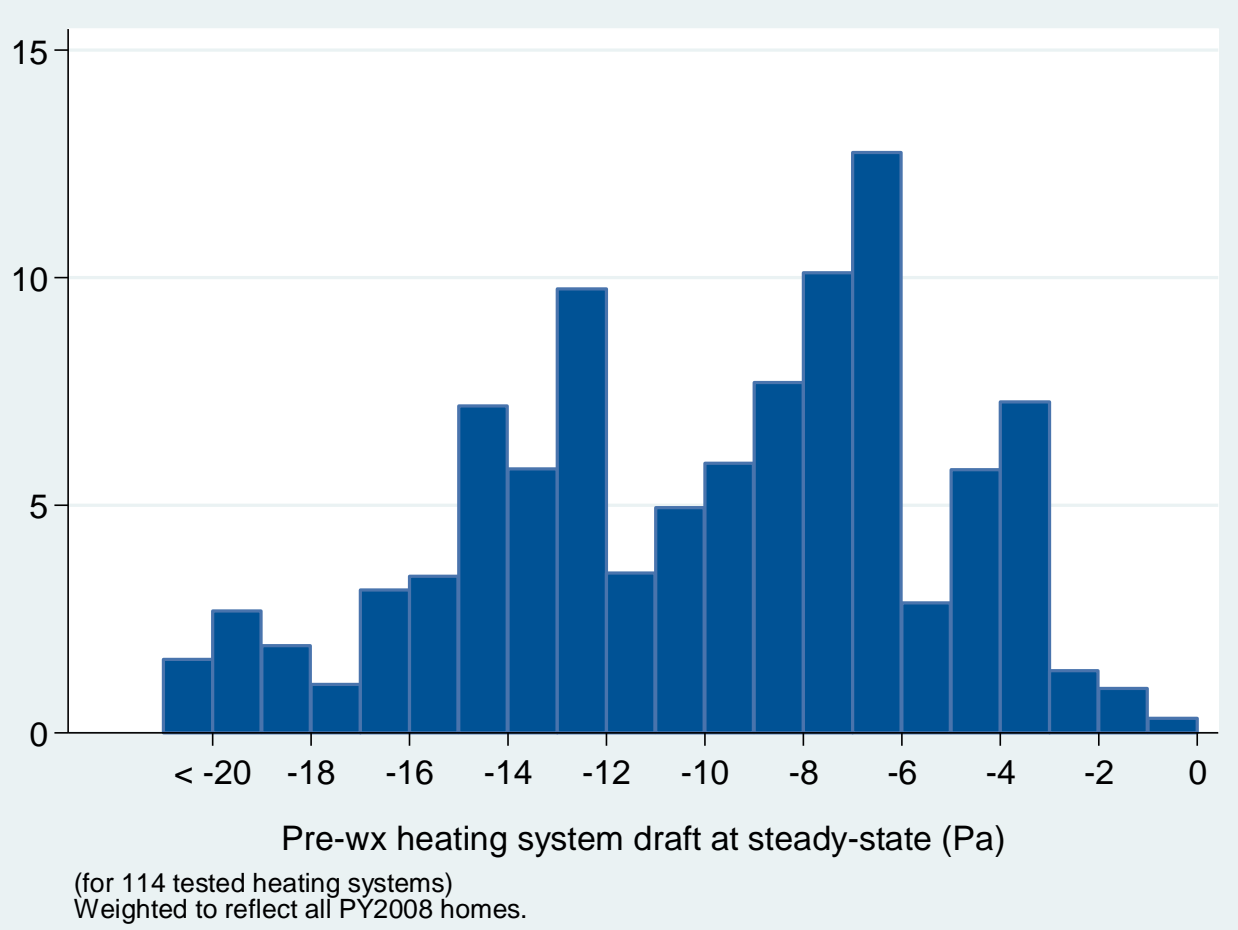

Fig. 2. Pre weatherization heating system draft.

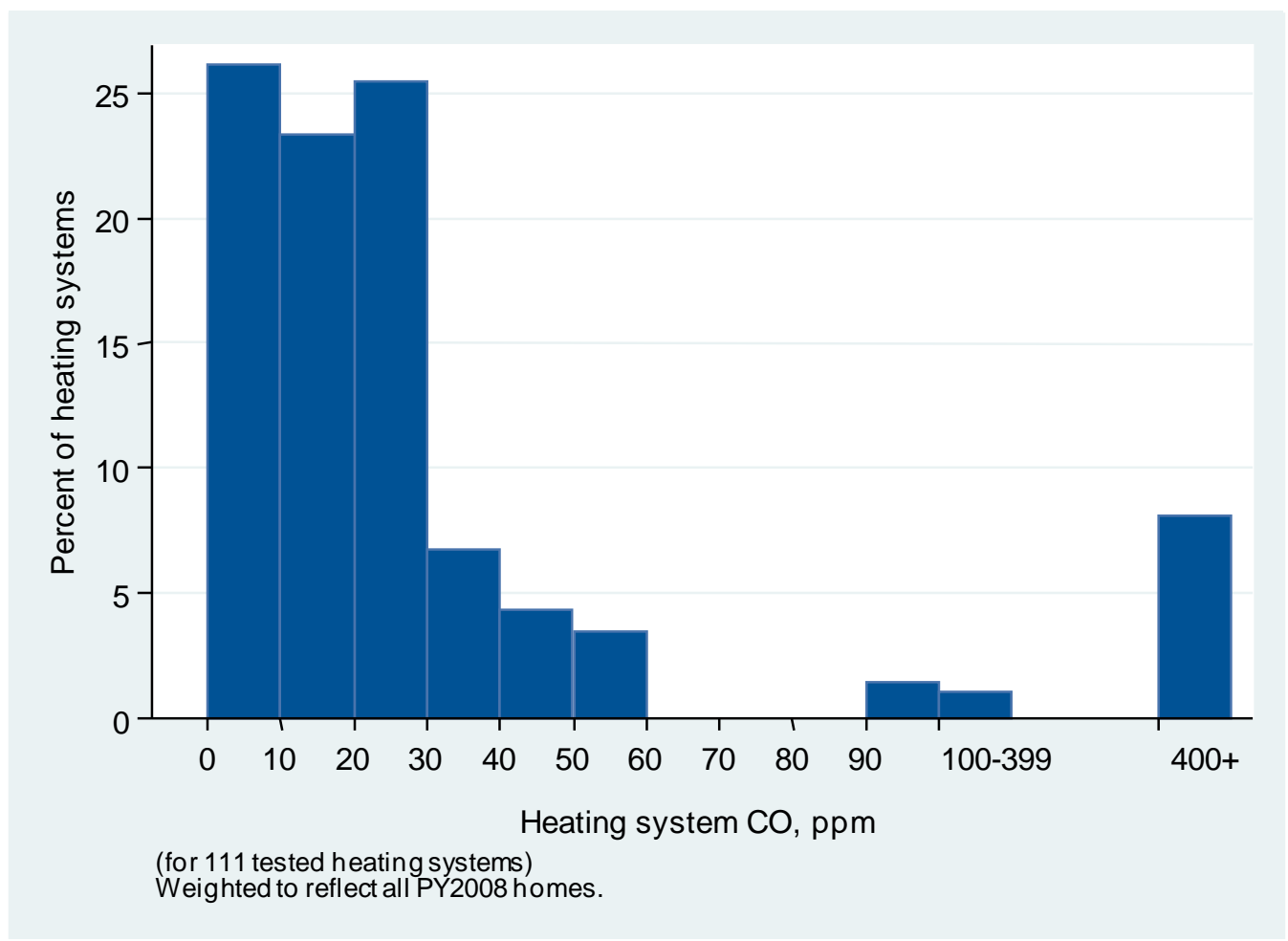

Fig. 3. Pre-weatherization heating system CO. 
Following weatherization, all of the measured remaining atmospheric systems in the treatment group $(n=48)$ had acceptable draft pressure per the BPI protocol, with a comparable distribution to the preweatherization values. Natural-vent systems in the control group $(n=39)$ showed a similar distribution.

Only one of the remaining natural-vent heating systems had measured $\mathrm{CO}$ above $400 \mathrm{ppm}$ following weatherization, and that test result is suspicious: the heating system in question had tested at only $1 \mathrm{ppm}$ prior to weatherization, no work was performed on the system, and the local weatherization agency was unable to replicate the test reading after being notified of the post-weatherization test result. The heating system (in a Pennsylvania home) was receiving gas directly from a natural gas well, which may have affected the reading in question.

\subsubsection{Water Heaters}

Water heater venting was categorized in a manner similar to that for space heating systems, using a classification of natural-draft for systems that include a draft hood and are susceptible to combustion products spillage. The study sample indicates that between 40 and 50 percent of weatherization homes have stand-alone, fuel-fired, natural-vent water heaters (located indoors) prior to weatherization.

Draft measurements made on 200 such systems showed that (on a weighted basis) about 85 percent had draft pressures that met or exceeded the BPI standard, while the remaining 15 percent did not pass the BPI requirements for adequate draft (Fig. 4). ${ }^{12}$ (As an estimate of the proportions in the larger population of weatherization homes, these figures have an estimated uncertainty of \pm 4 percentage points at a 90 percent confidence level.) Ten systems in the sample were recorded as showing positive draft pressure under the conditions tested, indicating either persistent combustion products spillage, or an error in testing or recording of data.

None of the water heaters included in the analysis data set showed carbon monoxide concentrations in the vent greater than $100 \mathrm{ppm}$ (Fig. 5). ${ }^{13}$

About 12 percent of the atmospheric water heaters in the treatment group were replaced during weatherization. Following weatherization, all remaining natural-vent water heaters with test results (85 treatment-group sites and 50 control-group sites) passed the BPI draft test protocol, including 9 systems that were not replaced and had failed the pre-weatherization draft test. The latter result is not entirely unexpected: it is possible for a water heater with marginal draft to fail under one set of conditions and pass under another.

Post weatherization testing revealed no water heaters in either group that produced $\mathrm{CO}$ in excess of 100 ppm.

\footnotetext{
${ }^{12}$ This analysis excludes 6 water heaters that were serviced - and one water heater that was replaced - prior to the first site visit.

${ }^{13}$ However, one water heater at a control-group site was measured as having a carbon monoxide concentration of 1,650 ppm during the pre-weatherization site visit. Communication of this potentially hazardous situation to the local agency resulted in early weatherization, and the site was ultimately dropped for unrelated reasons.
} 


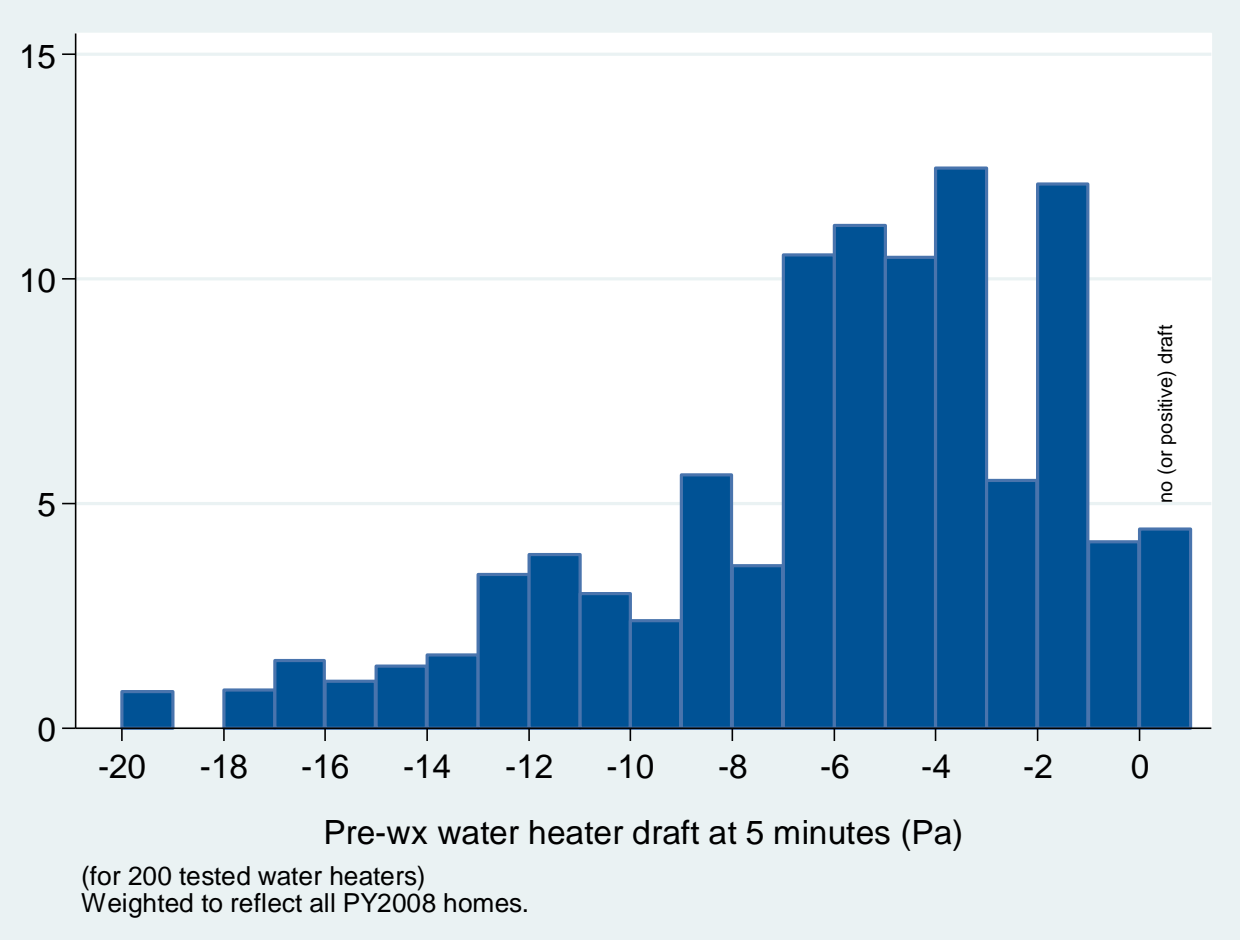

Fig. 4. Pre-weatherization water heater draft.

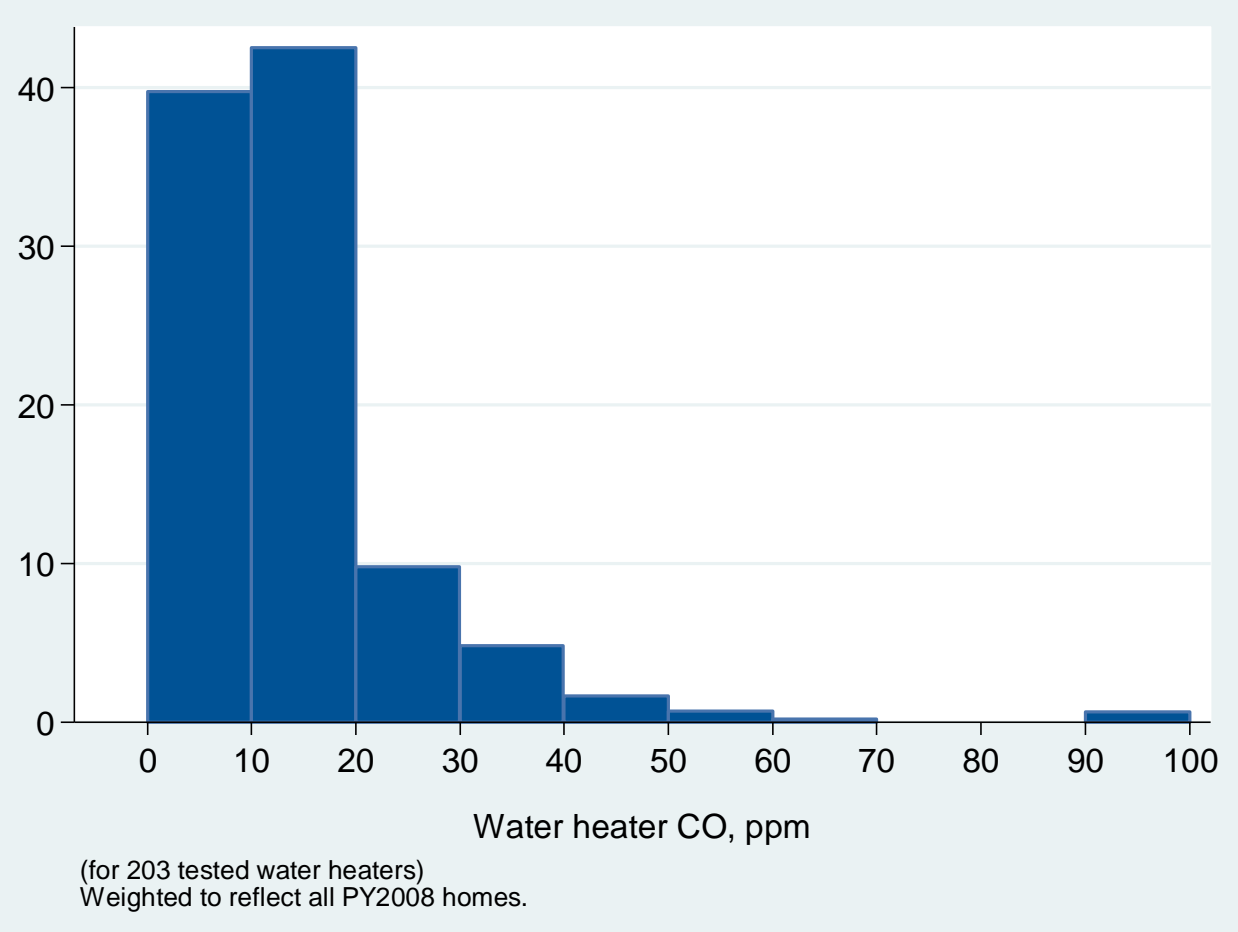

Fig. 5. Pre-weatherization water heater $\mathrm{CO}$ production. 


\subsubsection{Ranges and Ovens}

Because they are not directly vented to the outdoors, carbon monoxide produced by ranges and ovens is directly introduced to the indoor environment, in contrast to vented systems like water heaters and space heating systems. Because of this, many weatherization agencies include range and oven CO testing as part of their health and safety diagnostic protocol. Data from the Sampled Agencies Detailed Program Information Survey indicates that about 40 percent of local weatherization agencies measured cooking stove CO as part of their diagnostic procedures in PY08. Remedies for carbon monoxide produced by ranges and ovens include cleaning burners, adjusting oven air shutters and outright replacement, though the last is not an allowable expense with DOE funds.

The study data suggest that slightly less than half ( $43 \pm 7$ percent) of ranges and ovens in single-family homes treated by the program are natural gas or propane models. Among these, natural gas ranges and ovens outnumber propane models by about 3 to 1 - though among mobile homes, there are about the same number of natural gas and propane units.

As part of the site visit protocol, pre- and post-weatherization measurements were made of carbon monoxide produced by fuel-fired ranges and ovens. For range burners, technicians set all burners to high, allowed them to warm up for three minutes, and then recorded carbon monoxide and oxygen levels six inches above the center of each burner. For ovens, technicians measured $\mathrm{CO}$ and $\mathrm{O} 2$ in the oven vent after a five-minute warm-up period.

Carbon monoxide readings from appliances like ranges and ovens often vary due to differences in how much the combustion products have been diluted at the point of measurement. To account for this, carbon monoxide readings are typically adjusted to an "air-free" basis, which removes the effects of dilution, and provides a consistent measurement. ${ }^{14}$ Air-free values are reported here. As a reference point, current federal standards require new ranges and ovens to produce no more than $800 \mathrm{ppm} \mathrm{CO}_{\text {air-free }}$.

\section{Ranges}

Range CO measurements were obtained for 215 of the 225 fuel-fired ranges in the study, of which 166 had both pre- and post-weatherization measurements. Pre-weatherization $\mathrm{CO}_{\text {air-free }}$ readings averaged about $140 \mathrm{ppm}$, but spanned from zero to more than 3,000 ppm (Fig. 6). The data suggest that among fuel-fired ranges, about two percent of burners (involving about four percent of ranges) produce more than 800 ppm air-free CO. ${ }^{15}$

On average, there were only minor changes in burner $\mathrm{CO}$ following weatherization: measured $\mathrm{CO}$ levels were slightly lower in both the treatment and control groups in the post-weatherization period, with no significance difference between the two groups.

However, the substantial scatter for pre- and post-weatherization measurements on the same burner (Fig. 7) suggest that the reliability of measurements made in this way is low. In the control group, the median range burner had a post-weatherization $\mathrm{CO}$ measurement that differed from the preweatherization reading by about 33 percent. Moreover, the data show that the differences were somewhat larger when different technicians made the pre- and post-weatherization measurements. It is likely that small differences in where (and how) the measurements were made led to substantial differences in

\footnotetext{
${ }^{14}$ The adjustment to air-free $\mathrm{CO}$ is: $\mathrm{CO}_{\text {air-free }}=20.9 /(20.9-\mathrm{O} 2) * \mathrm{CO}_{\text {raw }}$, where $\mathrm{O} 2$ is expressed in percent. Because of the unreliability associated with this calculation when $\mathrm{O} 2$ readings are close to the ambient level of $20.9 \%, \mathrm{CO}_{\text {air-free }} \mathrm{Was}_{\text {calculated }}$ only for cases where the $\mathrm{O} 2$ was $\leq 20 \%$.

${ }^{15}$ This figure drops to less than one percent of all single-family homes when the more than half of homes with electric ranges is factored in.
} 
recorded air-free CO. Procedures that better control for these differences have been developed (Karg, 2001), but were too time intensive for the field protocol here.

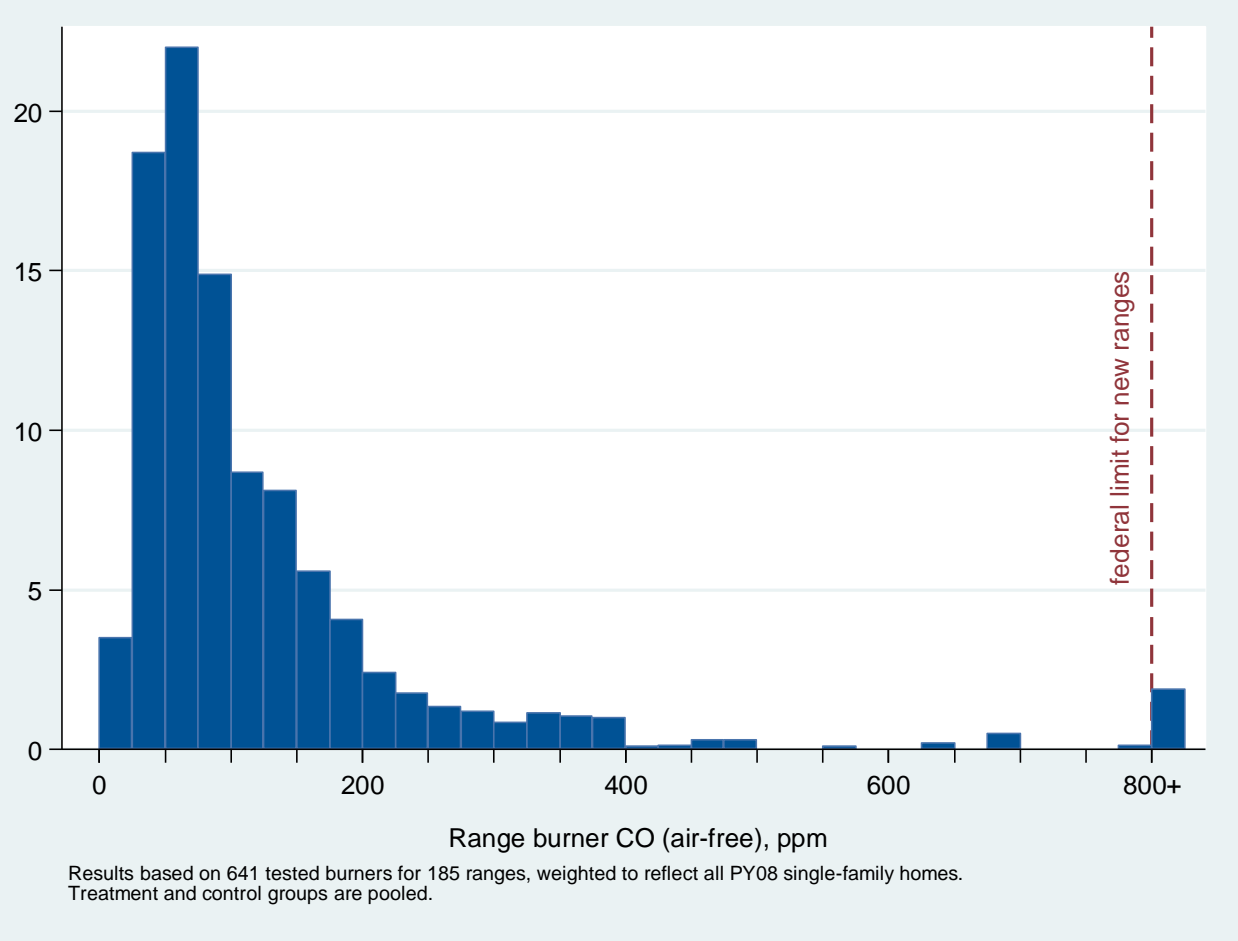

Fig. 6. Pre-weatherization carbon monoxide production by range burners.

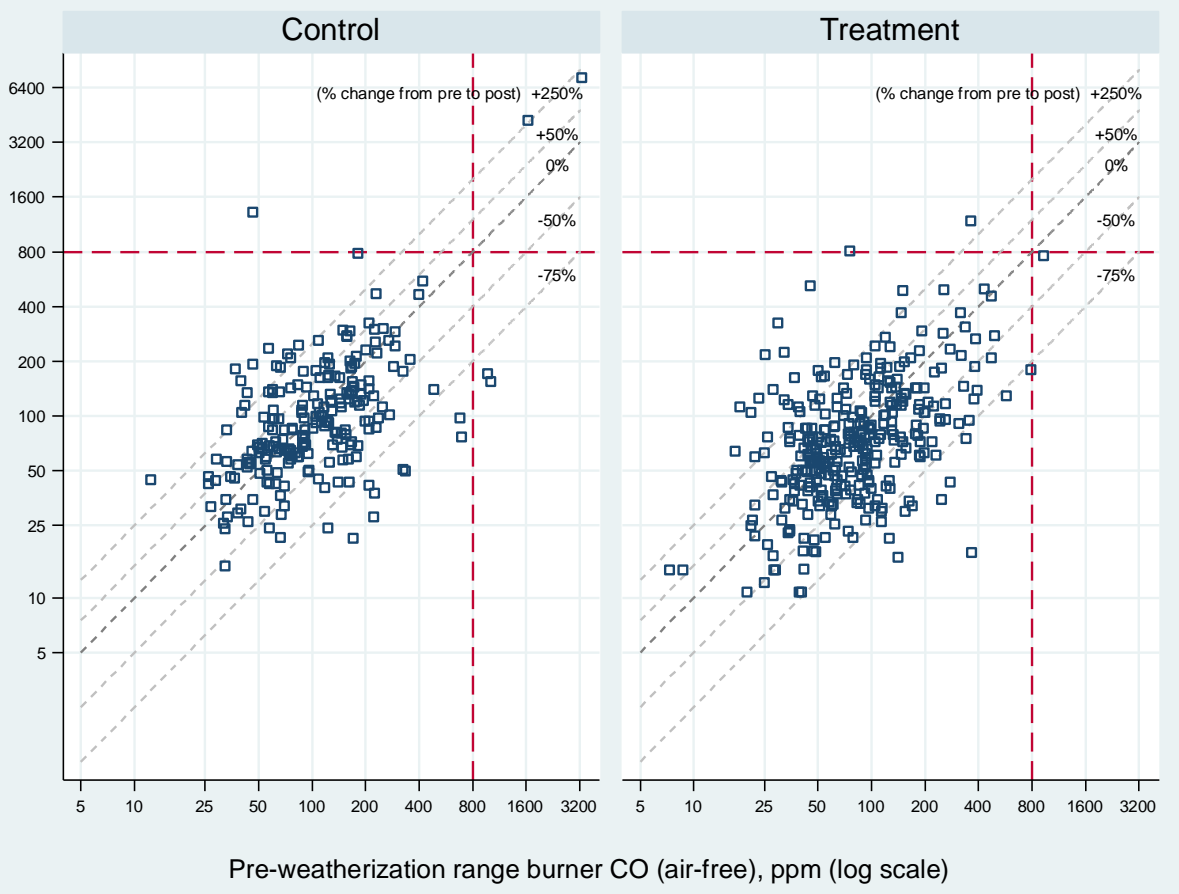

Fig. 7. Pre- versus post-weatherization range burner carbon monoxide. 


\section{Ovens}

Carbon monoxide concentrations produced by ovens - where measurements were made on 162 of 225 fuel-fired units-were somewhat higher. The data suggest that prior to weatherization, $12 \pm 5$ percent of fuel-fired ovens produce more than $800 \mathrm{ppm}$ air-free $\mathrm{CO}$, with the average oven producing about half this level (Fig. 8).

As with range burners, there was considerable scatter between pre- and post-weatherization measured oven CO levels, suggesting that the reliability of the simple test protocol used here is low (Fig. 9). In addition, a few tested ovens showed very large changes in $\mathrm{CO}$ level: only one of these (with a reading of about 800 ppm pre-weatherization and 20 post-weatherization) was traceable to an oven that was replaced at the time of weatherization. Data from the local weatherization agencies indicated only two oven replacements and two range/oven cleanings among the treatment group.

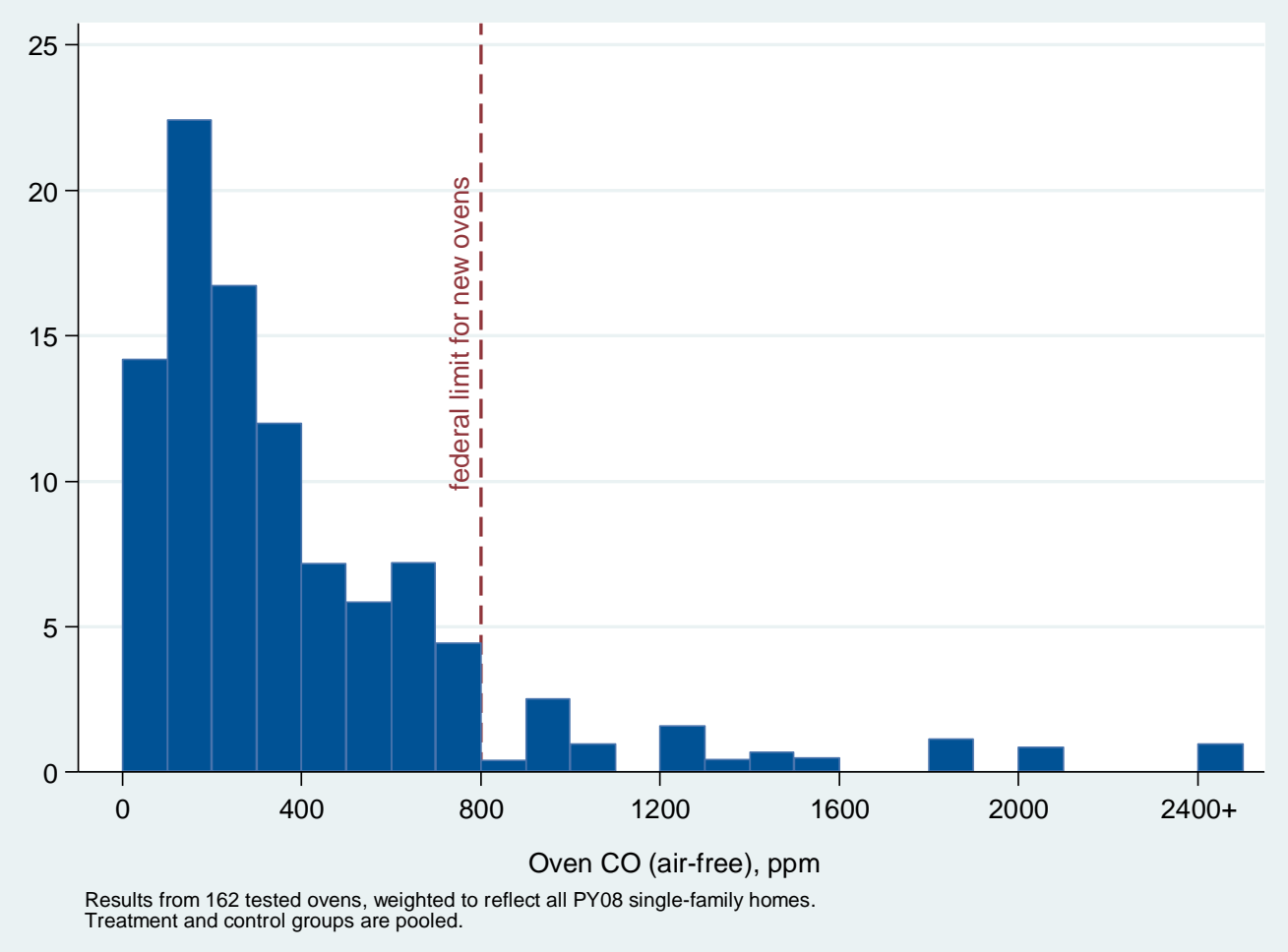

Fig. 8. Pre-weatherization carbon monoxide production by ovens. 


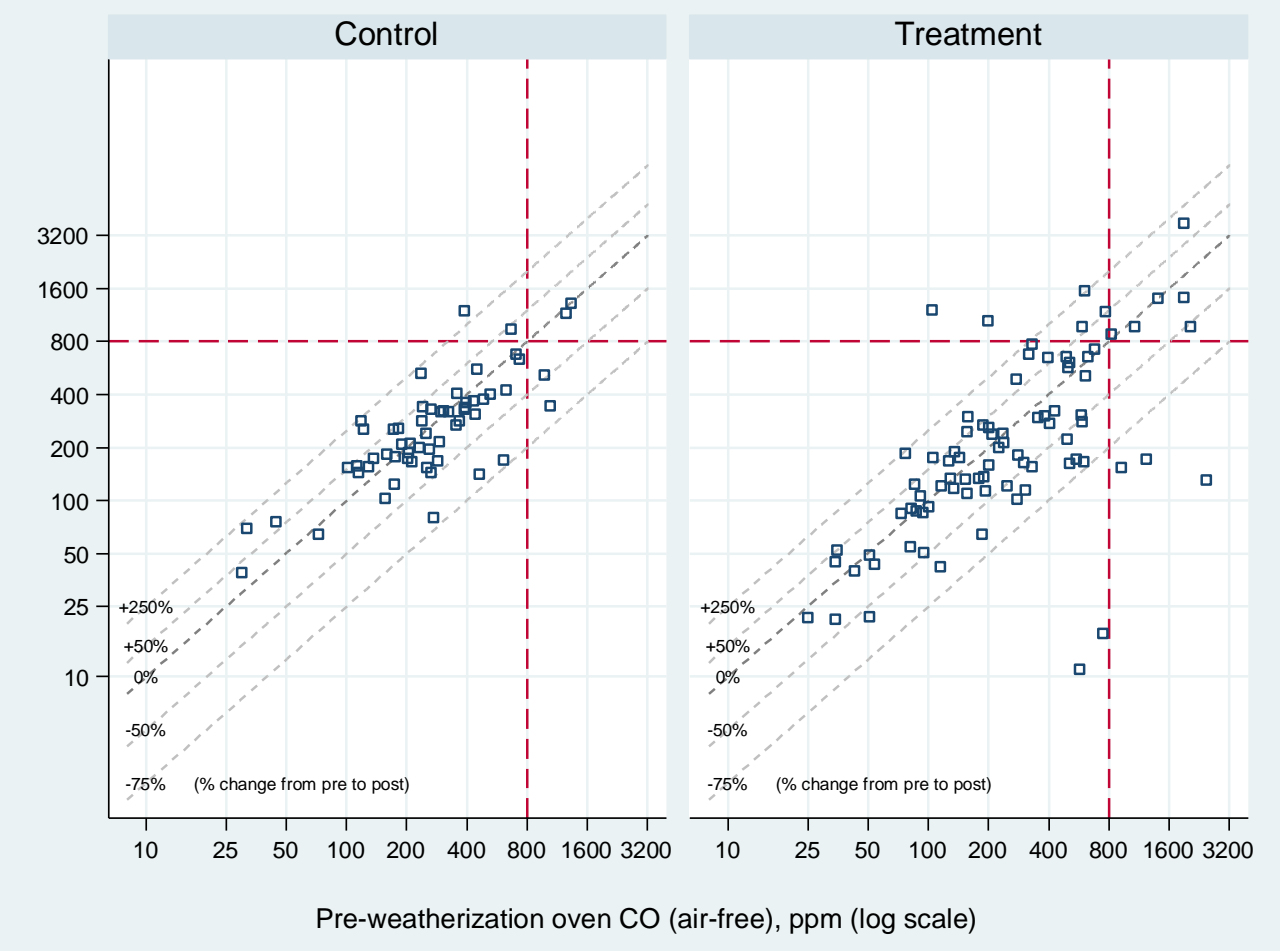

Fig. 9. Pre- versus post-weatherization oven carbon monoxide.

\subsubsection{Indoor Ambient Carbon Monoxide Levels}

The pre-weatherization data indicate that indoor ambient $\mathrm{CO}$ levels remained below $5 \mathrm{ppm}$ all or most of the time for the majority of the study homes (Table 4). About one in ten homes experienced episodic CO "events" that peaked at $20 \mathrm{ppm}$ or higher (the highest level recorded was $90 \mathrm{ppm}$ ). Even among these homes, such events are relatively uncommon: the median home saw one such event about every two weeks.

A few homes had persistent (but low) levels of indoor CO. On a weighted basis, the study data suggest that about one in 25 homes has an indoor $\mathrm{CO}$ level that exceeded $5 \mathrm{ppm}$ for ten percent of the time or more (Table 4), but the data suggest that only about one in one hundred program homes exceeds this threshold regularly. The highest overall average $\mathrm{CO}$ level recorded in the pre-weatherization period was $19 \mathrm{ppm}$, in a home that was one of two in which ambient CO remained above $5 \mathrm{ppm}$ for the entire preweatherization monitoring period. ${ }^{16}$

The treatment and control groups exhibited similar proportions, and there were no significant changes in these parameters following weatherization (Table 5).

The data for all homes with either episodic or persistently elevated recorded CO were reviewed in detail. A detailed accounting of this investigation is included in Appendix B. Summarizing here, the findings suggest that attached garages may be the cause of individual indoor ambient $\mathrm{CO}$ events, but are rarely

\footnotetext{
${ }^{16}$ Spot measurements of outdoor CO levels were also made during the first and third site visit. These measurements showed zero outdoor $\mathrm{CO}$ for all but six homes in the pre-weatherization period and three homes in the post-weatherization period. All of the non-zero readings were in the range of 1-2 ppm, and were not associated with sites where persistently elevated indoor CO was observed.
} 
related to long-term low levels of $\mathrm{CO}$ in these homes. Gas ranges and furnaces were the most likely causes of persistent elevated CO levels. ${ }^{17}$ Two examples of cases where elevated indoor CO could be clearly tied to a causative factor are presented below. In both cases, weatherization work appeared to resolve the $\mathrm{CO}$ issue.

Table 4. Pre-weatherization peak indoor ambient $\mathrm{CO}$ and $\mathrm{CO}$ persistence.

\begin{tabular}{lrrr}
\hline & & $\begin{array}{c}\text { Percent of } \\
\text { homes }\end{array}$ \\
\hline Peak observed CO level (ppm) & $<5$ & $59 \%$ & \pm 5 \\
& $5-9$ & $20 \%$ & \pm 3 \\
& $10-19$ & $12 \%$ & \pm 2 \\
& $20+$ & $9 \%$ & \pm 3 \\
Percent of time $>5$ ppm & & & \\
& 0 & $64 \%$ & \pm 6 \\
& $1-9 \%$ & $33 \%$ & \pm 5 \\
$10-49 \%$ & $2 \%$ & \pm 1 \\
$50+\%$ & $1 \%$ & \pm 1 \\
\hline
\end{tabular}

Results are weighted to reflect the population of single-family homes treated by the program in PY08.

Treatment and control groups are pooled. $n=496$

\pm values are approximate $90 \%$ confidence intervals.

Table 5. Pre- and post-weatherization peak indoor ambient $\mathrm{CO}$ and $\mathrm{CO}$ persistence, by group.

\begin{tabular}{lrrrccc}
\hline & & \multicolumn{2}{c}{ Treatment Group } & \multicolumn{2}{c}{ Control Group } \\
\cline { 3 - 7 } & & $\begin{array}{c}\text { Pre } \\
(\mathbf{n = 3 1 0})\end{array}$ & $\begin{array}{c}\text { Post } \\
(\mathbf{n = 2 6 9 )}\end{array}$ & $\begin{array}{c}\text { Pre } \\
(\mathbf{n = 1 8 6})\end{array}$ & $\begin{array}{c}\text { Post } \\
(\mathbf{n = 1 6 1})\end{array}$ \\
\hline Peak observed CO level (ppm) & $<5$ & $58 \% \pm 6$ & $57 \% \pm 6$ & $60 \% \pm 6$ & $62 \% \pm 6$ \\
& $5-9$ & $21 \% \pm 5$ & $24 \% \pm 5$ & $18 \% \pm 4$ & $20 \% \pm 6$ \\
& $10-19$ & $13 \% \pm 3$ & $13 \% \pm 3$ & $11 \% \pm 5$ & $11 \% \pm 5$ \\
& $20+$ & $8 \% \pm 3$ & $6 \% \pm 3$ & $11 \% \pm 6$ & $7 \% \pm 4$ \\
Percent of time $>5$ ppm & & & & & \\
& 0 & $65 \% \pm 6$ & $64 \% \pm 6$ & $64 \% \pm 7$ & $69 \% \pm 7$ \\
& $1-9 \%$ & $33 \% \pm 6$ & $31 \% \pm 6$ & $33 \% \pm 8$ & $27 \% \pm 7$ \\
& $10-49 \%$ & $2 \% \pm 1$ & $3 \% \pm 2$ & $3 \% \pm 2$ & $2 \% \pm 2$ \\
& $50+\%$ & $<1 \% \pm 1$ & $1 \% \pm 1$ & $<1 \% \pm 1$ & $2 \% \pm 1$ \\
\hline
\end{tabular}

Results are weighted to reflect the population of single-family homes treated by the program in PY08.

\pm values are approximate $90 \%$ confidence intervals.

\section{Example of CO from an Attached Garage}

The CO logger data from one study home in Pennsylvania (Site 9626-I20 in Appendix C) showed periodic spikes in indoor ambient $\mathrm{CO}$ prior to weatherization (Fig. 10). Further investigation showed that the home in question had a tuck-under garage (Fig. 11), and that there was no door separating the garage

\footnotetext{
${ }^{17}$ Oven operation was tracked for some sites, but these did not generally overlap with homes with elevated ambient indoor CO. Also, it was beyond the scope of the study to track operation of range hoods.
} 
space from the remainder of the basement. A new doorway was constructed as part of the weatherization work for the home, and the post-weatherization CO data showed an apparent reduction in indoor ambient $\mathrm{CO}$ events. Since the home had electric heat and no other combustion appliances, it seems likely that the source of the $\mathrm{CO}$ was automobile exhaust that was mitigated following weatherization.

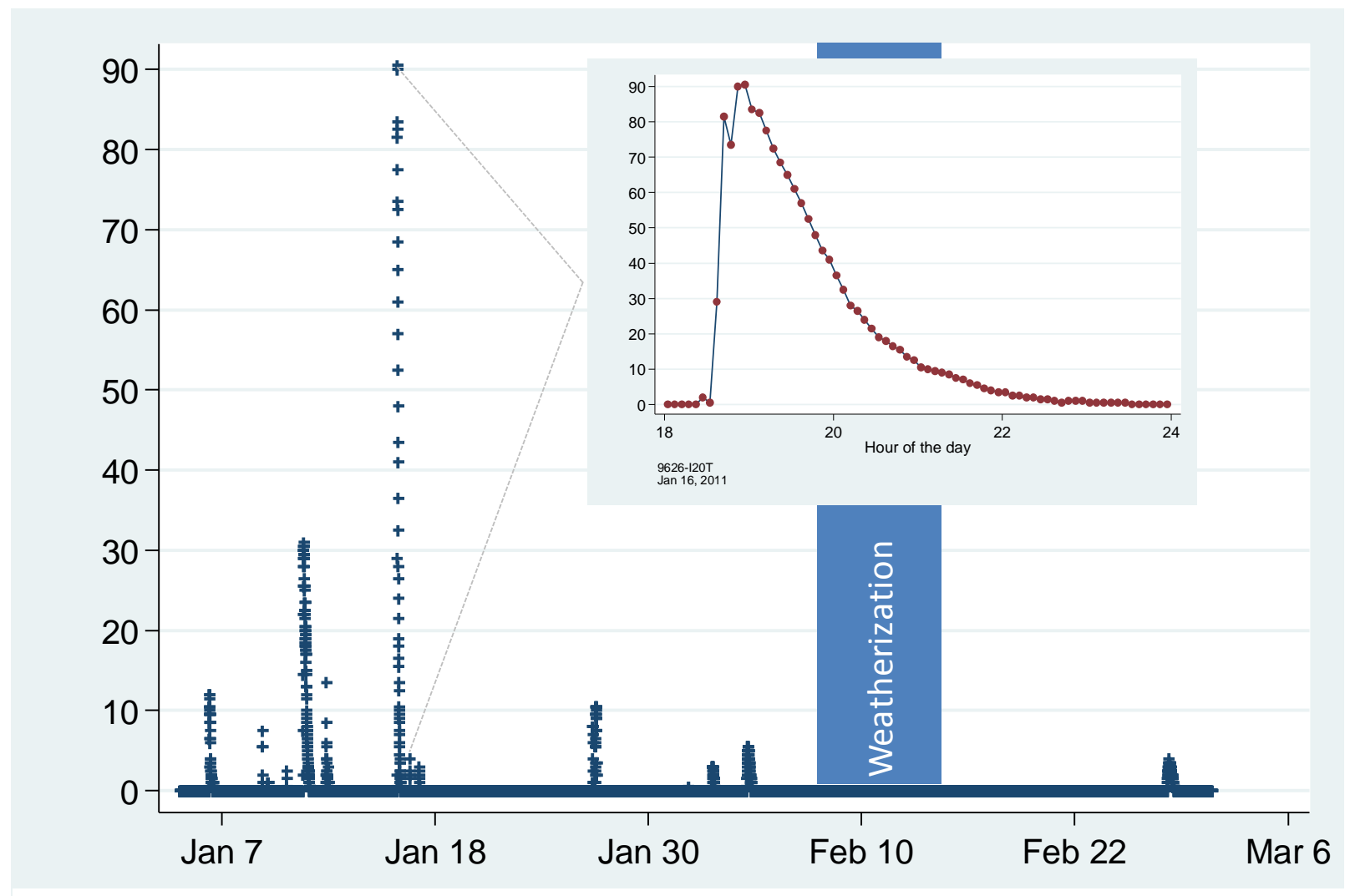

Fig. 10. Five-minute indoor ambient CO data for Site 9626-I20, with expanded view of January 16th event.

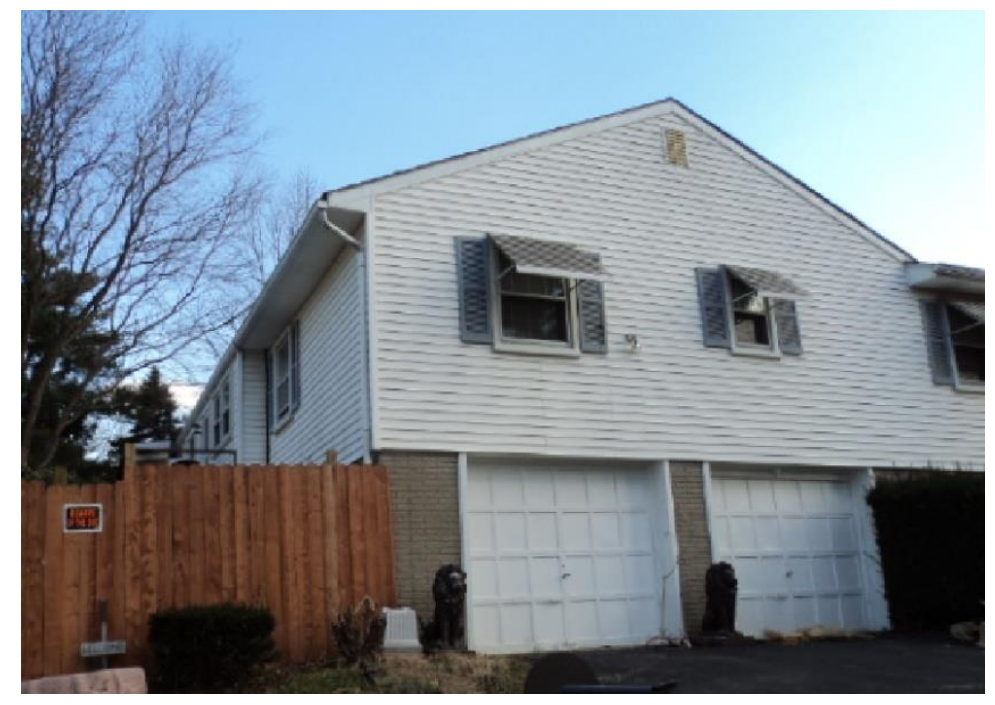

Fig. 11. Tuck-under garage at Site 9626-I20. 


\section{Example of CO from a Furnace}

The CO logger data from a site in Ohio (Site 5431-I05 in Appendix C) showed persistent indoor ambient $\mathrm{CO}$ with a periodic cycle to it at about the same frequency as a typical furnace cycling rate (Fig. 12). The pre-weatherization combustion test for the natural-draft gas furnace had to be terminated early because operation of the furnace raised the $\mathrm{CO}$ level in the ambient area around the unit beyond the 35ppm safety threshold. Indoor $\mathrm{CO}$ levels dropped to near zero when the furnace was replaced by weatherization with a sealed-combustion unit (Fig. 13).

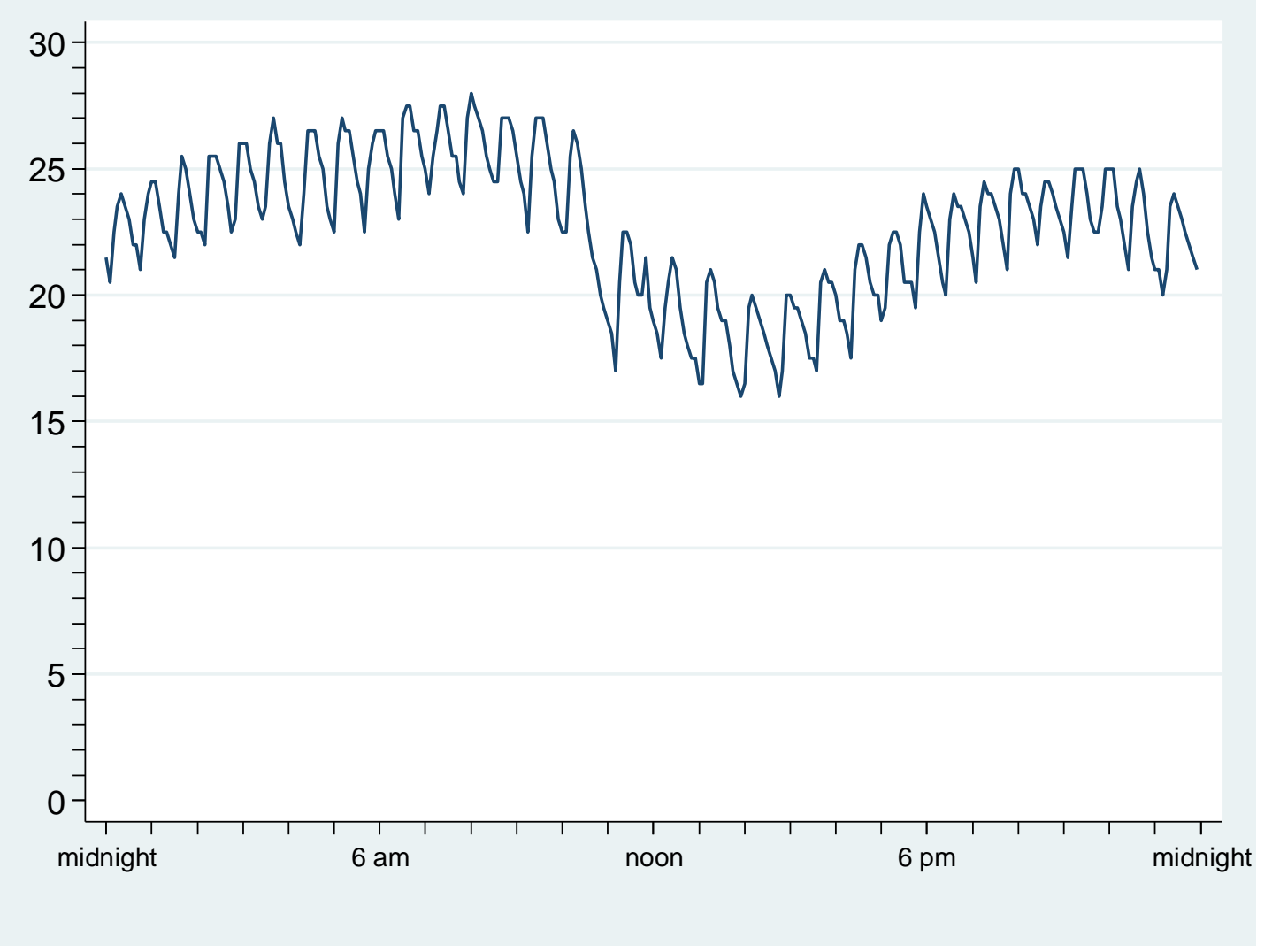

Fig. 12. Indoor ambient CO at Site 5431-I05 on January 25, 2011. 


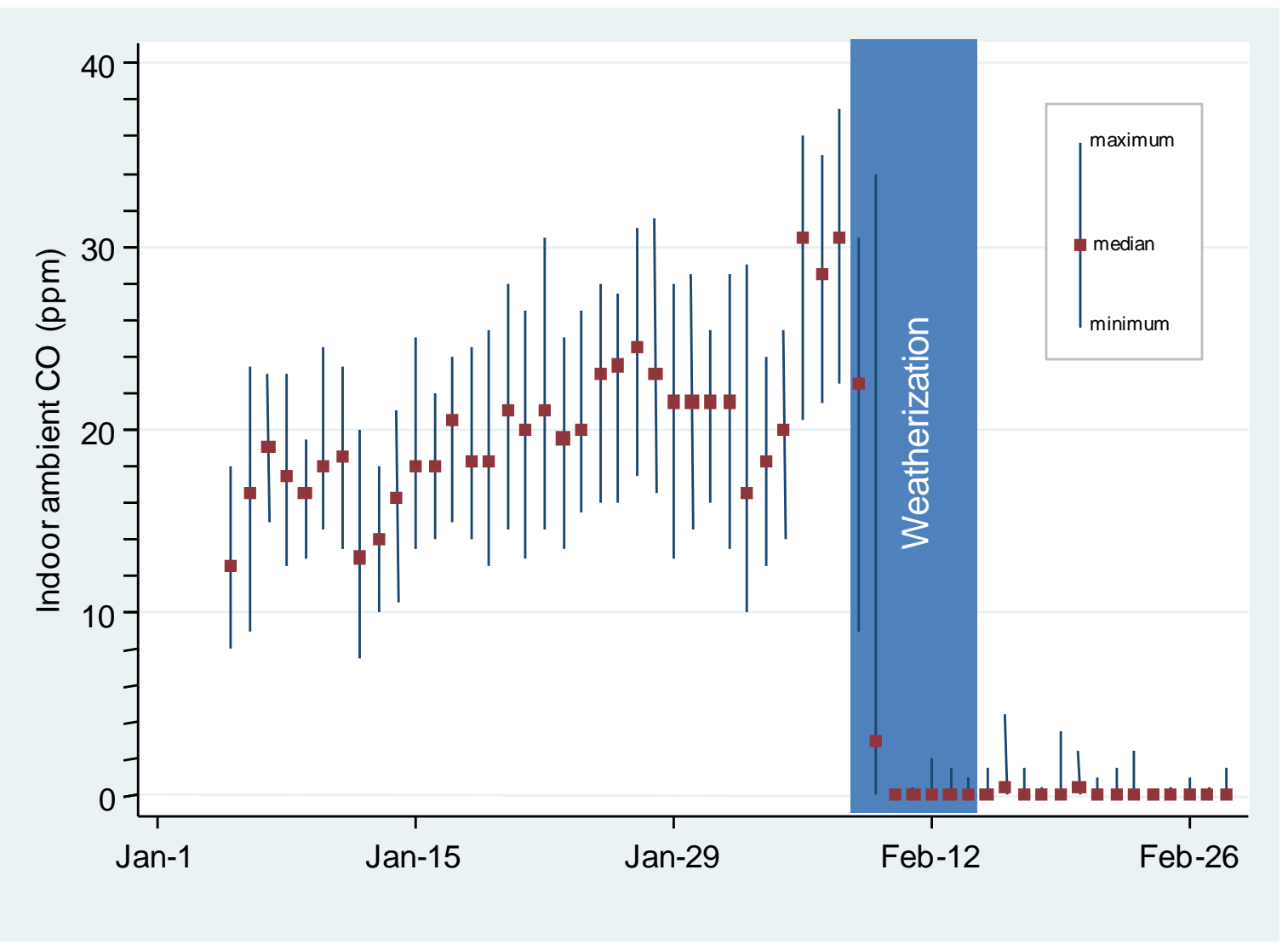

Fig. 13. Daily range and median indoor ambient CO level at Site 5431-I05.

\subsection{RADON}

Radon is an odorless radioactive gas that is a natural decay product of uranium. Radon in most homes originates in natural rock and soil formations and enters homes when soil gas infiltrates through cracks and holes in building foundations. The U.S. Environmental Protection Agency (EPA) and the World Health Organization (WHO) both recognize radon as the second leading cause of lung cancer after cigarette smoking (EPA 2011, WHO 2009).

Much research regarding radon in homes has been conducted since the 1970s. An overview of the literature on this topic is provided in Appendix C.

In the context of weatherization, radon is a potential concern because air-sealing work can reduce air exchange rates and thereby increase radon levels in homes. Also natural draft combustion appliances in basements provide some effective ventilation of theses spaces from the constant chimney effect they induce: radon levels may increase when weatherization replaces these devices with sealed combustion appliances that do not use house air for combustion. On the other hand, below-grade sealing work by weatherization may reduce the entry of radon into homes, and duct sealing work could reduce the undesirable distribution of radon in below-grade spaces to the remainder of the home. 
In the US, radon is typically measured in pico Curies per liter (pCi/L). ${ }^{18}$ The EPA (2009) recommends action if a long-term test - or the average of two short-term tests - shows a radon level of $4 \mathrm{pCi} / \mathrm{L}$ or higher, but EPA also notes that any radon exposure carries some risk for lung cancer.

Many homeowners do not know the radon level of their home, which can vary significantly from one home to another within the same neighborhood. Moreover, radon remediation typically costs about $\$ 1,000$, and thus represents a potential affordability issue for the low-income households served by the program.

The EPA has classified all US counties into three radon zones according to their estimated potential for indoor radon. Table 6 shows the estimated national proportions of single-family weatherization homes by zone and type of housing, and is derived from information about local weatherization agency service territories, county population data and data on PY08 program production. The data suggest that most homes in the program in PY08 are in EPA Zone-1 (high radon potential) and Zone-2 (moderate radon potential) counties, but about one in five homes is in a Zone-3 (low radon potential) county. It is also noteworthy that about one in four homes treated by the program is a mobile home: these are thought to be less susceptible to radon intrusion due to their typically well-ventilated foundation spaces.

Table 6. Estimated PY08 single-family WAP housing-unit proportions by radon zone and housing type.

\begin{tabular}{rccc}
\hline & \multicolumn{2}{c}{ Housing type } & \\
\cline { 2 - 3 } EPA Radon Zone & site-built & mobile home & Total \\
\hline High (Zone 1) & $32 \%$ & $9 \%$ & $41 \%$ \\
Moderate (Zone 2) & $28 \%$ & $10 \%$ & $38 \%$ \\
Low (Zone 3) & $16 \%$ & $5 \%$ & $21 \%$ \\
Total & $76 \%$ & $24 \%$ & $100 \%$ \\
\hline
\end{tabular}

The sample design for the study included an over-sample of homes in high radon areas in order to better measure the impact of weatherization on radon levels in these areas; however, overall averages that follow are weighted to reflect the actual distribution of homes in the program in PY08.

\subsubsection{Pre-Weatherization Radon Levels}

The study protocol called for measuring radon levels on the first floor of each home, as well as in basements and crawlspaces - though measurements were not made in the foundation spaces below mobile homes. The radon level for each home was characterized based on the test result for the lowest occupied level of the home: data gathered on homes that were sampled for the study indicate that about half of single-family homes in the program have basements and about a third of these are occupied. ${ }^{19}$ Thus, basement radon levels, which generally had higher measured radon levels, are relevant for about one in six homes.

Study technicians were trained to deploy and retrieve 7-day, activated charcoal radon test canisters with diffusion barriers before and after weatherization. ${ }^{20}$ The canisters were provided — and analyzed — by the EPA Radiation and Indoor Environments Laboratory in Las Vegas, Nevada. As a quality control check,

\footnotetext{
${ }^{18}$ The international unit for radiation concentrations is Becquerels per meter cubed $\left(\mathrm{Bq} / \mathrm{m}^{3}\right)$. One pCi/l equals $37 \mathrm{~Bq} / \mathrm{m}^{3}$.

${ }^{19}$ For the study, we defined an occupied basement to be one reported by the household to be occupied for 8 or more hours per week.

${ }^{20}$ Actual test periods ranged from 4 to 9 days, and were within exposure limits provided to us by EPA. Canisters were analyzed by the EPA's Radiation and Indoor Environments Laboratory in Las Vegas, Nevada within 12 days of being sealed on-site.
} 
duplicate canisters were also deployed in about 6 percent of cases, and a blank (sealed) canister was deployed in about 3 percent of cases. ${ }^{21}$ These checks revealed no issues with the testing procedures.

It should be noted that radon levels are known to vary significantly over short periods of time (e.g., Chiayu Lin, 1999-also see Appendix C). While this makes comparing short-term pre- and postweatherization test results uncertain for any individual home, the goal of the study was to assess the average impact of weatherization on indoor radon levels, and the sample was designed to include a enough homes to be able to overcome the variability inherent in short-term testing and thus detect fairly small effects from weatherization.

Moreover, the random assignment of homes into treatment and control groups that were geographically balanced and tested concurrently provides the ability to remove the influence of extraneous (e.g., weatherinduced) influences on radon concentrations that tend to affect all homes between the pre- and postweatherization test periods. Without this aspect of the study design, it would be difficult to separate seasonal and weather effects from the effect of weatherization.

One limitation of the study is that the testing was conducted entirely under closed-home conditions during the heating season, and therefore measured radon levels from the study cannot be taken as reflective of annual indoor radon levels among program homes, since radon has a known seasonality in heatingdominated climates (Nero, 1990 - also see Appendix C). ${ }^{22}$ However, the study can provide a useful indicator of the relative change in radon levels associated with weatherization during the heating season.

Figure 14 shows the weighted distribution of the pre-weatherization test results, and Table 7 summarizes the data. The results confirm that the highest average heating-season radon levels occur among site-built homes in EPA Zone 1 counties, and the lowest average levels are found among mobile homes and homes in EPA Zone 3 counties. No significant differences were seen in average radon levels between homes with self-reported smokers and non-smokers.

\footnotetext{
${ }^{21}$ Results for 97 duplicate canisters agreed with the primary canister to within $0.5 \mathrm{pCi} / 1$ in 88 percent of cases, and agreed to within $1.0 \mathrm{pCi} / \mathrm{l}$ in 95 percent of cases. All but one of the 56 blanks deployed came back with a radon result below the detection limit of $0.5 \mathrm{pCi} / \mathrm{l}$; the remaining blank canister yielded a reading of $1.0 \mathrm{pCi} / \mathrm{l}$.

${ }^{22}$ As noted previously, 23 homes (16 treatment, 7 control) located in EPA Zone- 2 and Zone- 3 counties in three southern states were tested during the summer of 2011. These are excluded from the analysis here, because there was not good assurance that closed-home conditions was maintained during the testing periods. In brief, the highest test result (pre- or post-weatherization) for these sites was $1.7 \mathrm{pCi} / \mathrm{L}$, and about half fell below the test detection limit of $0.5 \mathrm{pCi} / \mathrm{L}$.
} 


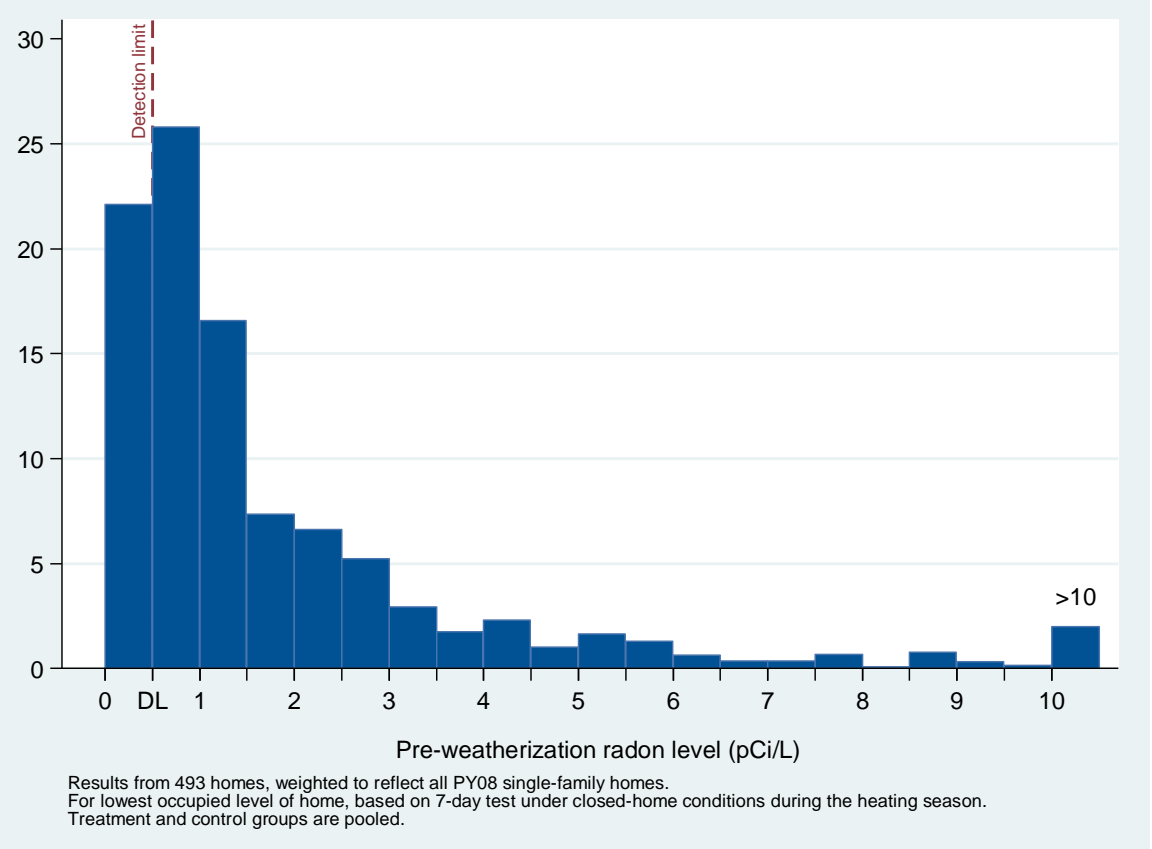

Fig. 14. Pre-weatherization radon level.

Table 7. Pre-weatherization radon level (lowest occupied level of home).

\begin{tabular}{|c|c|c|c|c|c|}
\hline & $\mathbf{N}$ & $\begin{array}{c}\% \text { below } \\
\text { detection } \\
\text { limit } \\
(0.5 \mathrm{pCi} / \mathrm{L})\end{array}$ & $\begin{array}{c}\text { Arithmetic } \\
\text { mean }^{\dagger} \\
(\mathbf{p C i} / \mathbf{L})\end{array}$ & $\begin{array}{c}\text { Geometric } \\
\text { mean }^{\dagger} \\
(\mathbf{p C i} / \mathbf{L})\end{array}$ & $\begin{array}{c}\text { Geometric } \\
\text { std. } \\
\text { deviation }^{\dagger}\end{array}$ \\
\hline Overall & 479 & $22 \% \pm 5$ & $1.9 \pm 0.3$ & $1.1 \pm 0.1$ & 2.8 \\
\hline \multicolumn{6}{|l|}{ By EPA radon zone } \\
\hline high (Zone 1) & 250 & $10 \% \pm 5$ & $2.5 \pm 0.3$ & $1.6 \pm 0.2$ & 2.9 \\
\hline moderate (Zone 2) & 182 & $20 \% \pm 6$ & $1.8 \pm 0.4$ & $1.0 \pm 0.2$ & 2.7 \\
\hline low (Zone 3) & 47 & $56 \% \pm 14$ & $0.6 \pm 0.2$ & $0.5 \pm 0.1$ & 1.7 \\
\hline \multicolumn{6}{|l|}{ By housing type } \\
\hline site built & 387 & $14 \% \pm 5$ & $2.3 \pm 0.3$ & $1.3 \pm 0.2$ & 2.9 \\
\hline mobile home & 92 & $46 \% \pm 14$ & $0.7 \pm 0.1$ & $0.5 \pm 0.1$ & 1.9 \\
\hline $\begin{array}{l}\text { Site-built homes in counties with high } \\
\text { potential (Zone 1) }\end{array}$ & 205 & $1 \% \pm 1$ & $3.0 \pm 0.4$ & $2.1 \pm 0.3$ & 2.6 \\
\hline \multicolumn{6}{|l|}{ Smoking (occupant survey) } \\
\hline \multicolumn{6}{|l|}{ Smoking rules in home } \\
\hline No smoking inside home $(71 \%)$ & 317 & $24 \% \pm 7$ & $1.9 \pm 0.3$ & $1.0 \pm 0.2$ & 2.8 \\
\hline Allowed some places/times (14\%) & 76 & $18 \% \pm 8$ & $1.7 \pm 0.3$ & $1.1 \pm 0.2$ & 2.7 \\
\hline Permitted anywhere (15\%) & 73 & $18 \% \pm 9$ & $2.3 \pm 0.6$ & $1.2 \pm 0.3$ & 3.0 \\
\hline \multicolumn{6}{|l|}{$\begin{array}{l}\text { Have you smoked at least } 100 \text { cigarettes } \\
\text { in your entire life? }\end{array}$} \\
\hline Yes $(58 \%)$ & 275 & $20 \% \pm 5$ & $2.0 \pm 0.3$ & $1.1 \pm 0.2$ & 2.9 \\
\hline No $(42 \%)$ & 192 & $25 \% \pm 8$ & $1.8 \pm 0.4$ & $1.0 \pm 0.2$ & 2.9 \\
\hline
\end{tabular}


Table 7. (continued)

\begin{tabular}{|c|c|c|c|c|c|}
\hline & $\mathbf{N}$ & $\begin{array}{c}\% \text { below } \\
\text { detection } \\
\text { limit } \\
(0.5 \mathrm{pCi} / \mathrm{L})\end{array}$ & $\begin{array}{c}\text { Arithmetic } \\
\text { mean }^{\dagger} \\
(\mathbf{p C i} / \mathrm{L})\end{array}$ & $\begin{array}{c}\text { Geometric } \\
\text { mean }^{\dagger} \\
(p C i / L)\end{array}$ & $\begin{array}{c}\text { Geometric } \\
\text { std. } \\
\text { deviation }\end{array}$ \\
\hline \multicolumn{6}{|l|}{ Do you now smoke cigarettes... } \\
\hline ...everyday? $(23 \%)$ & 109 & $24 \% \pm 8$ & $1.7 \pm 0.3$ & $1.0 \pm 0.2$ & 2.7 \\
\hline ...some days? $(5 \%)$ & 27 & $3 \% \pm 3$ & $2.2 \pm 0.5$ & $1.5 \pm 0.4$ & 2.3 \\
\hline ...not at all? $(72 \%)$ & 330 & $23 \% \pm 7$ & $2.0 \pm 0.4$ & $1.1 \pm 0.2$ & 2.9 \\
\hline In-home smoking ${ }^{\dagger \dagger}(20 \%)$ & 98 & $18 \% \pm 7$ & $1.8 \pm 0.4$ & $1.1 \pm 0.2$ & 2.7 \\
\hline
\end{tabular}

Results are for the lowest occupied level of the home under closed-home conditions, and are weighted to reflect the population of single-family homes treated by program in PY08.

Results below detection limit $(0.5 \mathrm{pCi} / \mathrm{L})$ set to 0.25 for calculation purposes.

Treatment and control groups are pooled.

\pm values are approximate $90 \%$ confidence intervals.

†The arithmetic mean is the sum of the observations divided by the number of observations. The geometric mean is the $n$th root of the product of $n$ observations, and is equivalent to $\exp (\operatorname{mean}(\ln (\mathrm{x})))$ less influenced by a small number of large values. The geometric standard deviation is equivalent to $\exp (\operatorname{stddev}(\ln (\mathrm{x})))$.

${ }^{\dagger}$ Based on the combination of smoking permitted in home and respondent-reported smoking on some days or everyday.

\section{Above-grade and foundation-level radon concentrations}

Figure 15 plots the measured pre-weatherization radon level for the first floor above grade against that measured in crawlspaces and basements for site-built homes with these foundation types. Above-grade radon levels are well-correlated with foundation levels, and generally lower in magnitude, as one would expect if the primary source of radon in homes is entry through foundations. The data indicate that on average, above-grade radon levels are about 70 percent that in foundation spaces. 


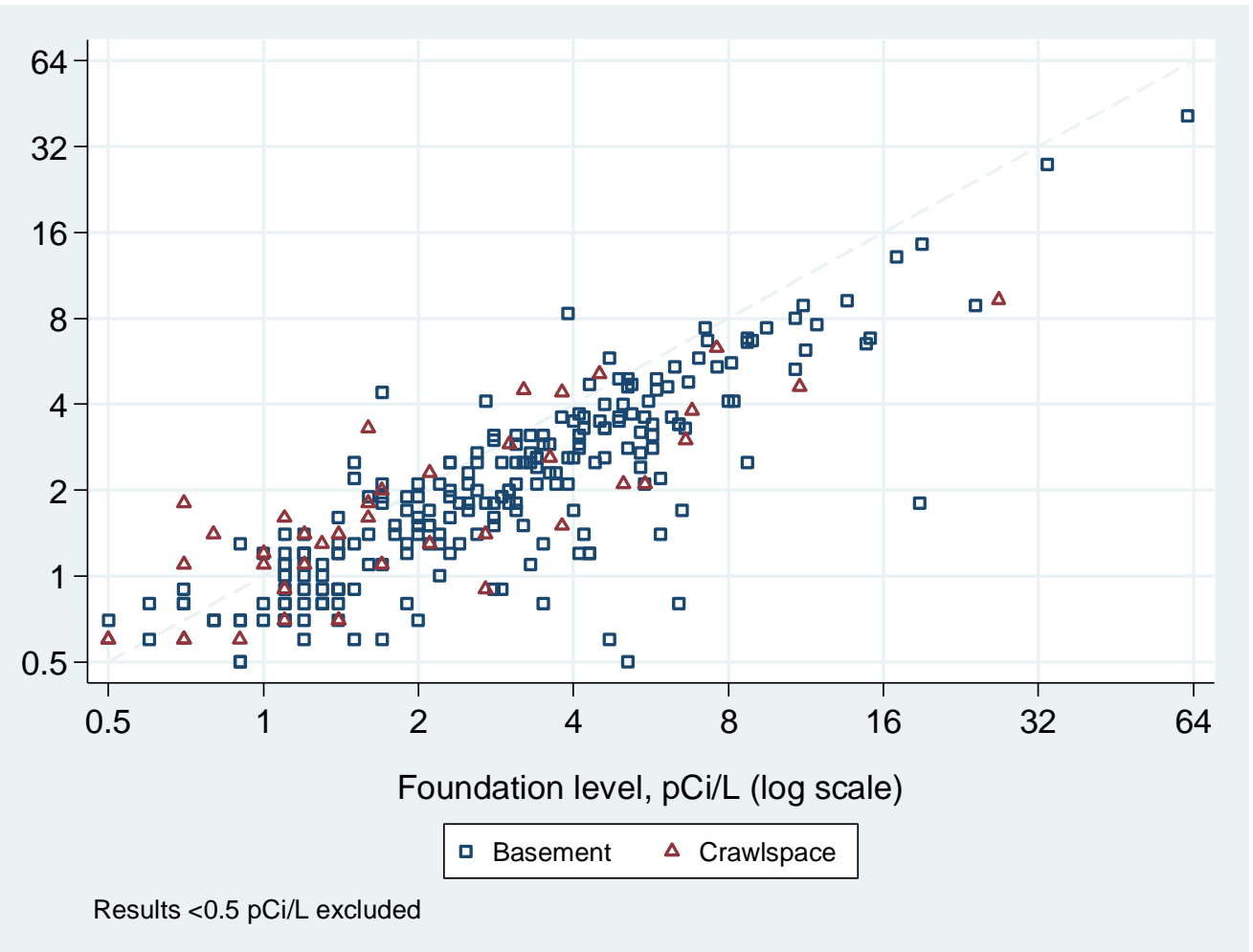

Fig. 15. Above-grade versus foundation-level radon level (pre-weatherization).

\section{Factors affecting radon levels}

Factors affecting observed pre-weatherization radon levels in study homes were explored with regression modeling. The analysis looked at how the natural logarithm of observed above-grade radon concentration varied across homes with the following variables:

- The natural logarithm of the predicted geometric mean indoor radon concentration for the county in which the home was located, from a county-level model of radon in homes developed by $\mathrm{LBNL}^{23}$

- Measured air leakage for the home prior to weatherization, expressed as the reciprocal of air changes per hour at 50 Pascals of depressurization ${ }^{24}$

- An indicator for whether the home was a mobile home

- An indicator for whether the home was single-family attached housing type

- The number of household members

- The number of stories for the home

- An indicator for the presence of a basement

\footnotetext{
${ }^{23}$ The LBNL model takes into account local geology as well as a large database of radon test results in actual homes. See http://eetd.lbl.gov/IEP/high-radon/hr.html

${ }^{24}$ The inverse of air leakage is used because (all other factors being equal) the impact of increased ventilation should be a decrease in indoor radon concentration.
} 
- An indicator for the presence of a crawlspace

- Average outdoor temperature during the test period

- Average barometric pressure during the test period

The results of this analysis (Table 8) show that several of these factors are highly statistically significant predictors of observed radon levels in the study homes. Taking those that are statistically significant at a 95 percent confidence level or higher, the model indicates that:

- radon levels are higher in homes that...

$\circ \quad$....are located in counties with higher predicted radon levels from the LBNL model.

- $\quad$ radon levels are lower in homes that...

- ...have higher measured air leakage, and therefore likely receive more natural ventilation.

○ ....are mobile homes, which typically have well-ventilated foundations.

- ...have more stories, which increases temperature-driven natural ventilation.

- ...have more household members, which may be associated with more door openings and general ventilation, but may also be because larger households are more likely to live in multi-story homes and less likely to live in a mobile home - and the model is not fully able to disentangle these factors.

○ ....are attached housing.

Table 8. Model of (log) pre-weatherization radon.

\begin{tabular}{|c|c|c|c|}
\hline Parameter & Coefficient & t-statistic & $\begin{array}{l}\text { Statistical significance level* - } \\
\text { significant at a } 90 \% \text { conf. level } \\
* * \text { - significant at a } 95 \% \text { conf. } \\
\text { level } \\
* * * \text { - significant at a } 99 \% \text { conf. } \\
\text { level }\end{array}$ \\
\hline \multicolumn{4}{|c|}{ Dependent variable: $\ln ($ radon level) - above-grade result, pre-weatherization } \\
\hline $\begin{array}{l}\mathrm{Ln}(\mathrm{LBNL} \text { county predicted } \\
\text { geometric mean radon }), \mathrm{pCi} / \mathrm{L}\end{array}$ & 0.628 & 7.24 & $* * *$ \\
\hline 1/ACH@50 & 3.83 & 3.31 & $* * *$ \\
\hline Mobile home & -0.884 & -5.04 & $* * *$ \\
\hline Attached, site-built home & -0.569 & -2.45 & $* *$ \\
\hline Stories above grade & -0.219 & -2.20 & $* *$ \\
\hline Presence of a basement in a site-built home & 0.154 & 1.05 & \\
\hline Presence of a crawlspace in a site-built home & 0.0413 & 0.15 & \\
\hline Number of household members & -0.0801 & -2.87 & $* * *$ \\
\hline $\begin{array}{r}\text { Mean outdoor temperature } \\
\text { during test period }(F)\end{array}$ & 0.00273 & 0.66 & \\
\hline $\begin{array}{l}\text { Mean barometric pressure } \\
\text { during test period }(\mathrm{mb})\end{array}$ & 0.0151 & 1.75 & $*$ \\
\hline Presence of a sump pump & 0.112 & 1.04 & \\
\hline Presence of dirt foundation floor & 0.0140 & 0.05 & \\
\hline model constant & -15.05 & -1.72 & \\
\hline Regression statistics: $\mathrm{n}=322 ;$ adjusted $\mathrm{r}^{2}=0.355 ; \mathrm{F}$ sta & istic $=15.75$ & & \\
\hline
\end{tabular}




\subsubsection{Change in Radon Levels}

Post-weatherization radon test results were generally correlated with pre-weatherization test results (Fig. 16), and for most homes, the observed change in radon level was less than $1 \mathrm{pCi} / \mathrm{L}$ (Fig. 17).

Overall, radon levels increased by an average of $0.14 \pm 0.13 \mathrm{pCi} / \mathrm{L}$ in the treatment group after weatherization and decreased by $0.29 \pm 0.18 \mathrm{pCi} / \mathrm{L}$ in the control group (Table 9). This leads to an average net (treatment minus control) increase of $0.44 \pm 0.22 \mathrm{pCi} / \mathrm{L}$ in home radon levels based on arithmetic means. However, this average is affected by a small number of sites with high preweatherization radon levels and commensurately large changes in radon levels. When nine (of 447) sites with measured pre-weatherization radon levels above $10 \mathrm{pCi} / \mathrm{L}$ are excluded from the analysis, the average net change is reduced to $0.28 \pm 0.14 \mathrm{pCi} / \mathrm{L}$.

As Table 9 shows, the average increase is largest among site-built homes in EPA high-radon counties, and, conversely, is small and statistically insignificant among homes in EPA low-radon counties. Interestingly, the study suggests a weatherization-related decrease in radon levels in mobile homes that is statistically significant at about a 90 percent confidence level.

Studies dating to the late 1980s have looked at the impact of weatherization on radon concentrations in homes, with mixed conclusions: see "the Effects of Weatherization on Radon" in Appendix C for more details. While these studies often involved more detailed radon measurements and air exchange testing than the study described here, the current effort is notable in terms of its geographic diversity, large sample size and the use of randomization to control for non-program factors that affect radon levels.

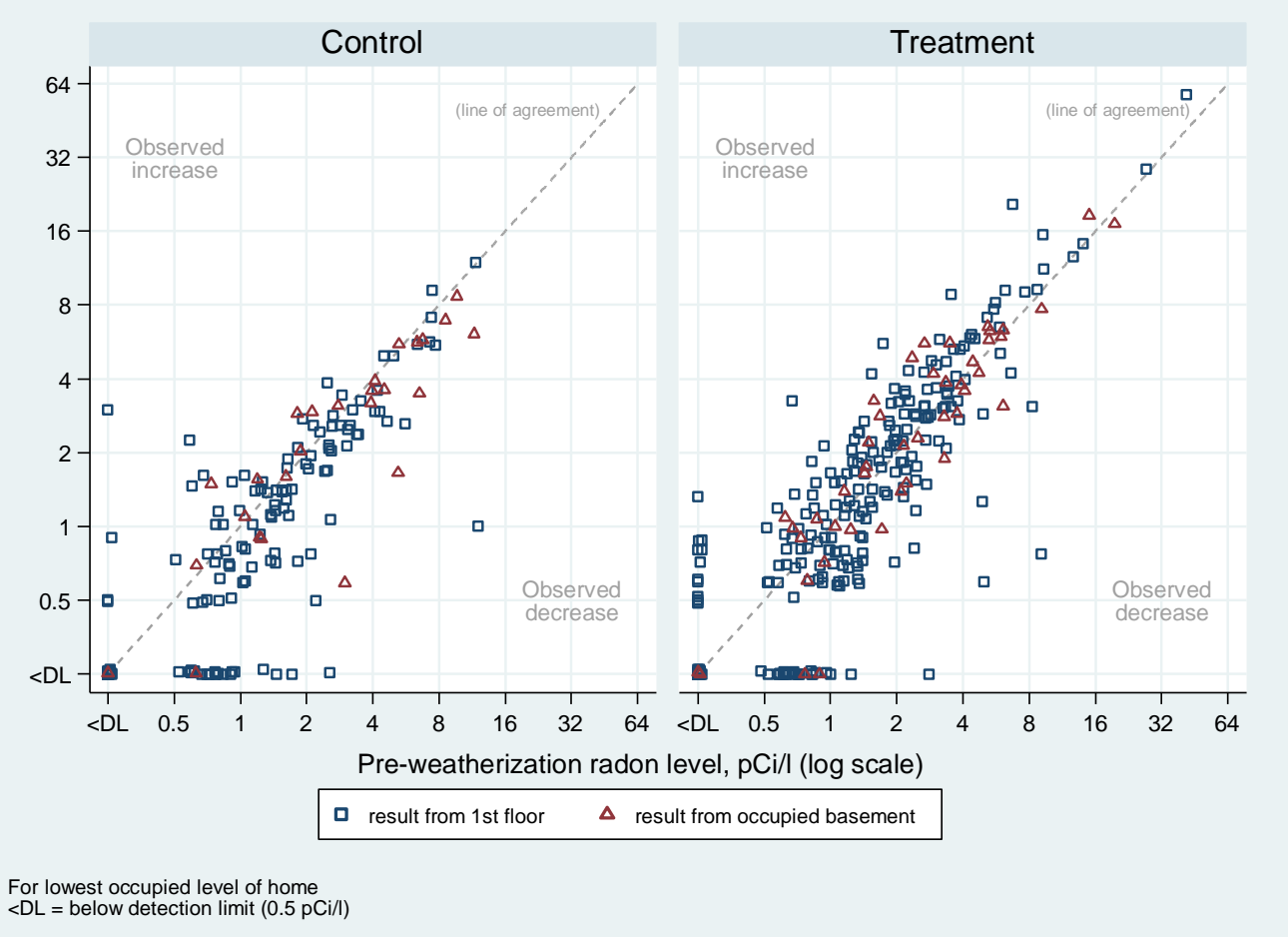

Fig. 16. Post- vs. pre-weatherization radon. 


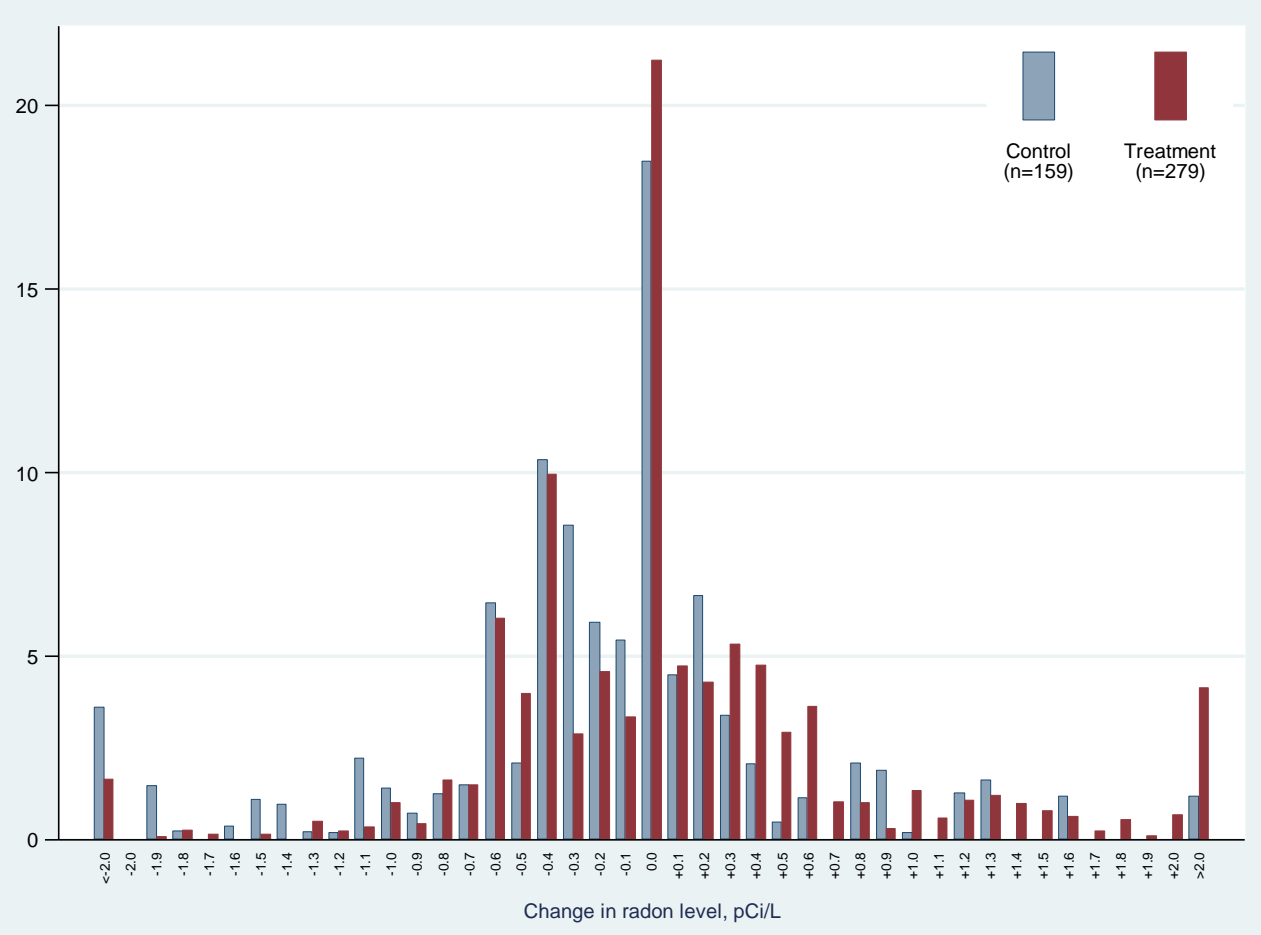

Fig. 17. Distribution of change in radon level.

Table 9. Net change in radon level, by EPA zone and housing type (arithmetic means).

\begin{tabular}{|c|c|c|c|c|c|c|}
\hline & & \multicolumn{2}{|c|}{ Pre-weatherization, $\mathrm{pCi} / \mathrm{L}$} & \multicolumn{3}{|c|}{ Change (Post - Pre), pCi/L } \\
\hline & $\mathbf{n}$ & $\begin{array}{c}\text { Treatment } \\
\text { Group } \\
(\mathbf{n}=\mathbf{2 8 5}) \\
\end{array}$ & $\begin{array}{l}\text { Control } \\
\text { Group } \\
(\mathrm{n}=162)\end{array}$ & $\begin{array}{c}\text { Treatment } \\
\text { Group }\end{array}$ & $\begin{array}{c}\text { Control } \\
\text { group }\end{array}$ & $\begin{array}{c}\text { Net } \\
\text { (treatment - } \\
\text { control) }\end{array}$ \\
\hline Overall & & & & & & \\
\hline all cases & 447 & $2.0 \pm 0.3$ & $1.9 \pm 0.3$ & $+0.14 \pm 0.13$ & $-0.29 \pm 0.18$ & $+0.44 \pm 0.18$ \\
\hline $\begin{array}{r}\text { preWX radon }<10 \\
\mathrm{pCi} / \mathrm{L}\end{array}$ & 438 & $1.6 \pm 0.2$ & $1.7 \pm 0.3$ & $+0.11 \pm 0.12$ & $-0.16 \pm 0.12$ & $+0.28 \pm 0.14$ \\
\hline $\begin{array}{l}\text { By EPA radon } \\
\text { zone }\end{array}$ & & & & & & \\
\hline $\begin{array}{r}\text { high } \\
\text { (Zone 1) }\end{array}$ & 234 & $2.4 \pm 0.4$ & $2.7 \pm 0.5$ & $+0.29 \pm 0.18$ & $-0.50 \pm 0.33$ & $+0.79 \pm 0.31$ \\
\hline $\begin{array}{r}\text { moderate } \\
\text { (Zone 2) }\end{array}$ & 170 & $2.3 \pm 0.6$ & $1.4 \pm 0.3$ & $+0.10 \pm 0.26$ & $-0.11 \pm 0.25$ & $+0.23 \pm 0.28$ \\
\hline $\begin{array}{r}\text { low } \\
\text { (Zone 3) }\end{array}$ & 43 & $0.6 \pm 0.2$ & $0.8 \pm 0.3$ & $-0.10 \pm 0.14$ & $-0.11 \pm 0.13$ & $+0.01 \quad \pm 0.20$ \\
\hline By housing type & & & & & & \\
\hline site-built & 362 & $2.4 \pm 0.3$ & $2.3 \pm 0.3$ & $+0.24 \pm 0.16$ & $-0.44 \pm 0.21$ & $+0.68 \pm 0.24$ \\
\hline mobile home & 85 & $0.8 \pm 0.2$ & $0.6 \pm 0.1$ & $-0.13 \pm 0.16$ & $+0.20 \pm 0.24$ & $-0.33 \pm 0.29$ \\
\hline $\begin{array}{l}\text { Site-built homes } \\
\text { in counties with } \\
\text { high potential } \\
\text { (Zone 1) }\end{array}$ & 192 & $2.8 \pm 0.4$ & $3.3 \pm 0.6$ & $+0.46 \pm 0.21$ & $-0.62 \pm 0.45$ & $+1.08 \pm 0.42$ \\
\hline
\end{tabular}

Results are for the lowest occupied level of the home under closed-home conditions, and are weighted to reflect the population of single-family homes treated by WAP in PY08.

Results below detection limit $(0.5 \mathrm{pCi} / \mathrm{L})$ set to 0.25 for calculation purposes.

\pm values are approximate $90 \%$ confidence intervals. 
To test whether the observed average increase in radon levels found here holds true across a range of preweatherization radon levels, homes with pre-weatherization radon levels between 1 and $10 \mathrm{pCi} / \mathrm{L}$ were divided into quintiles, and control-adjusted changes in radon levels within each quintile were examined. ${ }^{25}$ The results of this analysis show statistically significant net increases in radon levels for all but the lowest quintile (Table 10). This lends further credence to the notion that the averages presented in Table 9 are not driven solely by large changes in a few homes, but reflect a general increase in radon levels in homes associated with weatherization.

Table 10 suggests that the effect of weatherization tends to be at least roughly proportional to preweatherization radon levels: the upper four quintiles show point estimates for the net change in radon level that are in the range of 20 to 30 percent of the pre-weatherization level for the respective quintile. The results suggest that one can generally expect to see a relatively small absolute impact on radon in homes with low existing levels, and a larger absolute impact in homes with high existing levels.

Table 10. Net change in radon level for homes with pre-weatherization radon between 1 and $10 \mathrm{pCi} / \mathrm{L}$, by preweatherization quintile.

\begin{tabular}{lcccccc}
\hline \multirow{2}{*}{$\begin{array}{c}\text { Pre-weatherization } \\
\text { quintile } \\
\text { (pCi/L range) }\end{array}$} & Treatment Group & $\begin{array}{c}\text { Control } \\
\text { Group }\end{array}$ & $\begin{array}{c}\text { Treatment } \\
\text { Group }\end{array}$ & $\begin{array}{c}\text { Pre-weatherization } \\
\text { Control } \\
\text { group }\end{array}$ & $\begin{array}{c}\text { Change (Post - Pre) } \\
\text { (treatment - } \\
\text { control) }\end{array}$ \\
\hline $1^{\text {st }}$ & $(1.00-1.30)$ & $1.1 \pm 0.0$ & $1.1 \pm 0.0$ & $-0.13 \pm 0.12$ & $-0.10 \pm 0.11$ & $-0.03 \pm 0.16$ \\
$2^{\text {nd }}$ & $(1.31-1.70)$ & $1.4 \pm 0.0$ & $1.5 \pm 0.1$ & $0.04 \pm 0.17$ & $-0.27 \pm 0.13$ & $0.31 \pm 0.22$ \\
$3^{\text {rd }}$ & $(1.71-2.40)$ & $2.1 \pm 0.0$ & $2.0 \pm 0.1$ & $0.42 \pm 0.30$ & $-0.24 \pm 0.29$ & $0.66 \pm 0.42$ \\
$4^{\text {th }}$ & $(2.41-3.70)$ & $3.1 \pm 0.1$ & $2.8 \pm 0.2$ & $0.24 \pm 0.30$ & $-0.35 \pm 0.35$ & $0.59 \pm 0.46$ \\
$5^{\text {th }}$ & $(3.71-10.0)$ & $5.7 \pm 0.4$ & $5.8 \pm 0.6$ & $0.45 \pm 0.84$ & $-0.74 \pm 0.49$ & $1.18 \pm 0.98$ \\
\hline
\end{tabular}

Results are for the lowest occupied level of the home under closed-home conditions, and are weighted to reflect the population of single-family homes treated by the program in PY08.

\pm values are approximate $90 \%$ confidence intervals.

The observed decrease in radon levels in the control group also bears additional scrutiny. Radon levels in homes are known to be a complex interplay of radon migration through soil gas, soil gas intrusion into living spaces, and ventilation of those living spaces. Weather plays a role in all of these aspects through impacts of rain, frozen or snow-covered ground on soil gas movement, as well as temperature (and wind) impacts on basement pressure and natural ventilation rates.

The observed changes in radon levels in the control group were found to be related to outdoor temperature in a statistically-significant and meaningful way: sites that experienced decreases in outdoor temperature between the two test periods were associated with increases in radon levels, and, conversely, increases in outdoor temperature were associated with decreases in radon levels (Fig. 18). This result is consistent with the notion that colder weather increases the stack-effect depressurization of foundation spaces, which in turn increases the intrusion of soil gas into living spaces. It is true that the ventilation rate of buildings also increases in cold weather due to the increased stack effect, and that this should tend to reduce radon levels through dilution, but the data here suggest that the increased intrusion of radonbearing soil gasses-or other factors that are correlated with outdoor temperature-is the more dominant

\footnotetext{
${ }^{25}$ On a weighted basis, about half of the study homes had pre-weatherization radon test results that were in the range of 1 to 10 $\mathrm{pCi} / \mathrm{L}$, with nearly all of the remaining sites falling into the $<1 \mathrm{pCi} / \mathrm{L}$ category. Sites with pre-weatherization radon below $1 \mathrm{pCi} / \mathrm{L}$ were excluded for this particular analysis in order to focus on cases with readily detectable pre-existing radon levels. At the other end of the distribution, less than three percent of study homes had pre-weatherization radon levels above $10 \mathrm{pCi} / \mathrm{L}$ : these were removed to mitigate the influence of large outliers for this analysis.
} 
factor. Others (e.g., Arvela, 1995; Krewski et al., 2005-see Appendix C) have similarly posited an inverse relationship between outdoor temperature and radon levels within the heating season.

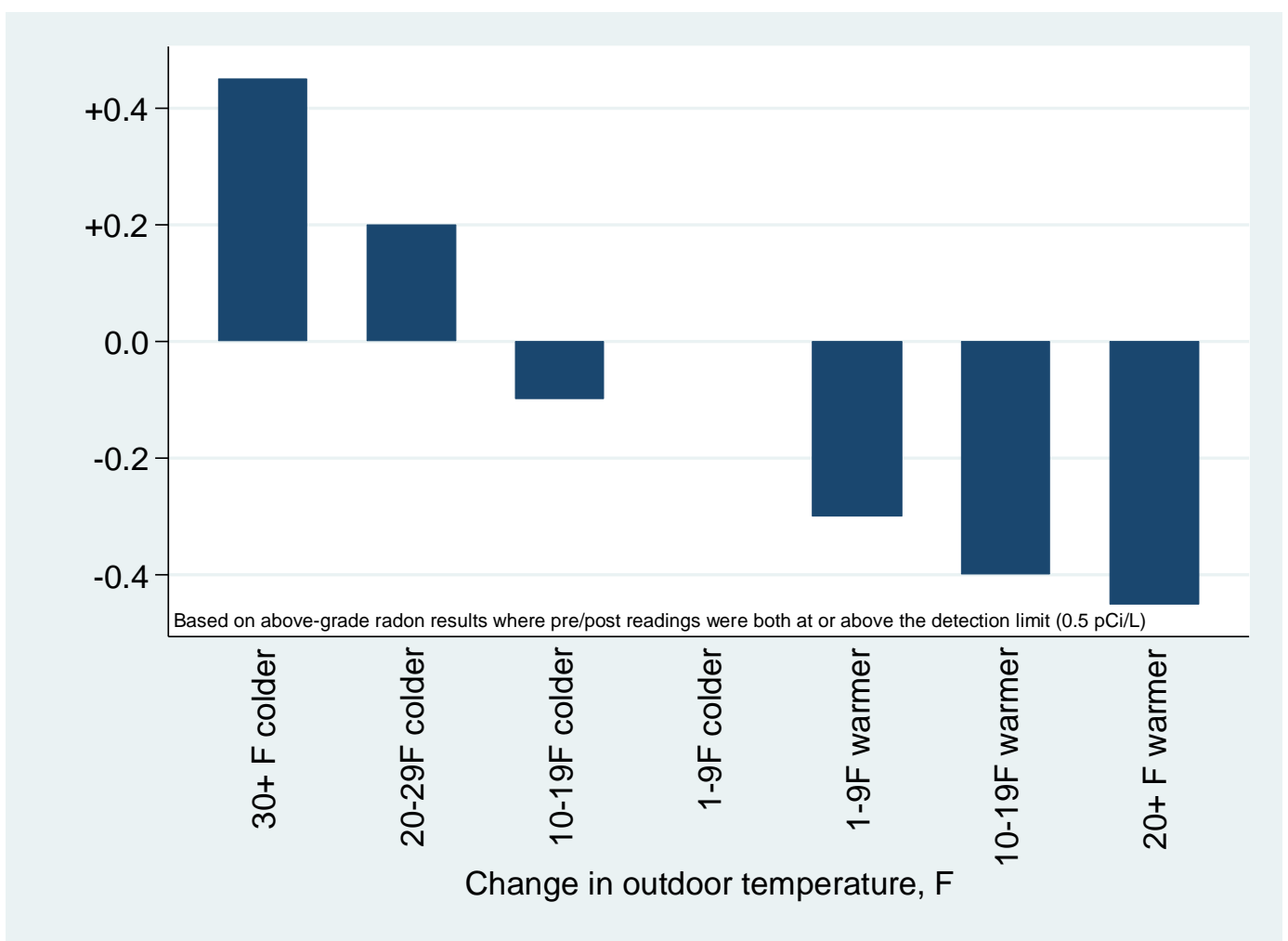

Fig. 18. Median change in control group radon level vs. change in outdoor temperature.

One might ask why outdoor temperature is a significant predictor of the change in radon level here, but it was not a good predictor in the prior model of pre-weatherization radon levels. The answer likely lies in the fact that the pre-weatherization model was a cross-sectional analysis of radon levels, in that it sought to address factors that result in a higher radon test result in one home and a lower test result in another. In that context, temperature is probably much less important than other factors in predicting radon levels. In contrast, an analysis of the change in radon level removes most of what makes radon levels different from home to home, and leaves weather as the most significant factor, especially among the control group homes where there was no intervening weatherization activity.

The staggered field deployment for the study meant that some sites had pre-weatherization tests performed in the fall and post-weatherization testing in deep winter, while other sites had preweatherization testing in winter and post-weatherization tests in late winter and early spring. While this made for a wide range in outdoor temperature differences across the study sample (from about $-40 \mathrm{~F}$ to $+30 \mathrm{~F}$ ), on a weighted average basis, outdoor temperatures were $3 \mathrm{~F}$ warmer during post-weatherization testing, and hence the overall average radon levels in the control group were slightly lower in the postweatherization period.

\section{Factors affecting changes in radon level}

In a fashion similar to our examination of factors that are predictive of pre-weatherization radon levels in homes, an assessment was also made of factors that predict pre/post changes in radon levels. As with the prior model, a number of factors were considered, in two general groups: 
- Weather changes

- Barometric pressure

- Fraction of days with rain

- Indoor/outdoor temperature difference

- Snow cover

- Changes in house characteristics from weatherization

- Air leakage and estimated natural ventilation

- Replacement of a natural or induced draft furnace with a sealed combustion model

- Addition of a ground cover

- Sealing of foundation cracks

- Addition of mechanical ventilation (continuous or non-continuous)

As noted above, radon entry into homes is an extremely complicated process. Rain and frozen ground affect the migration of radon through soil. This has the potential to force more radon into homes since the soil beneath homes is typically not saturated or frozen. Air pressure is also a potentially important parameter, because changes in air pressure can result in differential pressures across foundation structures and affect entry of soil gas into the home. There is also the consideration of stack effect. On the one hand, the greater stack effect during colder weather decreases below-grade air pressure, which then sucks more soil gas into the home. However, colder weather also increases the natural ventilation rate of a home, which tends to dilute pollutants such as radon.

Weatherization activities can affect radon levels by altering the ability of radon-bearing soil gas to enter the home, changing the rate at which radon gas is ventilated from the home, or both. Covering dirt foundation spaces and sealing foundation cracks can reduce the pathways by which radon enters the home in the first place and potentially reduce radon levels. General air sealing that tightens the home and reduces natural ventilation can potentially increase radon, while below grade air sealing - or air sealing that decreases air movement from foundation spaces to living areas - could reduce radon in occupied spaces. Similarly, the addition of mechanical ventilation may increase ventilation rates and potentially reduce radon concentrations, but may also further depressurize foundation spaces and increase soil-gas migration into the home. Replacing an atmospheric heating system in a basement with a sealed combustion unit may also impact ventilation rates, because an atmospheric appliance like a furnace effectively acts like a small, continuously-running exhaust fan in the space in which it is located - which, in the case of heating systems located in basements is the part of the home where radon concentrations are typically highest.

The overall ventilation rate of the home is undoubtedly an important factor in assessing changes in radon levels. While the study protocol did not provide for measurement of actual air change rates during the radon test periods (by means of tracer gas testing), measured air leakage data were available for most homes in the study. The artificial conditions under which air leakage is measured with a blower door makes it a relatively crude proxy for actual ventilation rates, but it's predictive power can be improved somewhat with a model that takes into account actual indoor and outdoor temperatures and other known factors about the home, such as height. Changes in radon level were evaluated as a function of predicted changes in natural infiltration using the enhanced model of natural ventilation in the American Society of Heating, Refrigerating and Air Conditioning Engineers (ASHRAE) Handbook of Fundamentals (ASHRAE 2009).

For some variables, it is appropriate to compare the change in radon level before and after weatherization with the arithmetic change in the parameter. However, for air leakage and infiltration, basic dilution principles would suggest that radon levels would move in relative proportion with the reciprocal of natural ventilation. Other house characteristics, which are binary (yes/no) variables, can be included in 
either type of analysis. After exploring a number of possible models, a hybrid approach was used: a model in which the dependent variable was $\operatorname{Radon}_{\text {post }} / \operatorname{Radon}_{\text {pre }}$ - to model relative changes in radon levels to relative changes in estimated air change rates - but that also included other variables that are more related to changes.

The analysis was confined to cases with both pre- and post-weatherization radon levels that were above the test detection limit of $0.5 \mathrm{pCi} / \mathrm{L}$. The analysis was also conducted using only the above-grade radon test result, so as not to confound the analysis with a mix of above-grade and below-grade tests.

The results of the model are shown in Table 11 for two runs: the first using all available data; and the second screened to remove cases where the post/pre ratio is less than 0.3 or more than 3.0. The latter run helps guard against outliers having a substantial impact on the results of the bulk of the sample. Only 8 homes were excluded from the analysis using the screened results.

Table 11. Model of post/pre radon level.

Dependent variable: Radon $_{\mathrm{pos}} / \mathrm{Radon}_{\mathrm{pre}}(\mathrm{pCi} / \mathrm{L}$, above-grade test result)

\section{$\underline{\text { Run 1: all cases included }}$}

\section{$\mathrm{ACHnat}_{\text {pre }} / \mathrm{ACHnat}$ post \\ Change in outdoor temperature, $\mathrm{F}$ \\ Change in fraction of days with rain during test period \\ Mobile home (binary) \\ Ground cover added to site-built home (binary) \\ Below grade sealing for site-built home (binary) \\ Continuous mechanical ventilation added (binary)}

Non-continuous mechanical ventilation or dryer venting added (binary)

Heating system replaced w/ sealed combustion model (binary)

model constant

Regression statistics: $\mathrm{n}=236$; adjusted $\mathrm{r}^{2}=0.135 ; \mathrm{F}$ statistic $=5.06$

\section{$\underline{\text { Run 2: restricted to } 0.3<\text { Radon }_{\text {post }} \text { Radon }_{\text {pre }} \leq 3.0 \text { (8 cases dropped) }}$}

$$
\begin{array}{r}
\text { ACHnat } \\
\text { Chre } / \text { ACHnat }_{\text {post }} \\
\text { Change in outdoor temperature, F } \\
\text { Ground cover added to site-built home (binary) } \\
\text { Below grade sealing for site-built home (binary) } \\
\text { Continuous mechanical ventilation added (binary) } \\
\text { Heating system replaced w/ sealed combustion model (binary) }
\end{array}
$$

Non-continuous mechanical ventilation or dryer venting added (binary)

Regression statistics: $\mathrm{n}=228 ;$ adjusted $\mathrm{r}^{2}=0.114 ; \mathrm{F}$ statistic $=4.25$

$\begin{array}{rrl}\text { Coefficient } & \text { t-statistic } & \\ 0.188 & 2.34 & * * \\ -0.00877 & -3.52 & * * * \\ -0.422 & -2.71 * * * \\ -0.0864 & -0.85 & \\ 0.114 & 1.16 & \\ -0.00982 & -0.13 & \\ -0.254 & -2.12 * * \\ 0.189 & 2.12 * * \\ 0.283 & -0.85 & \\ 0.774 & 7.31 * * *\end{array}$

$\begin{array}{rr}\text { Coefficient } & \mathrm{t} \text {-statistic } \\ 0.220 & 3.44 * * * \\ -0.009 & -4.66 * * * \\ -0.192 & -1.51 \\ -0.193 & -2.33 * * \\ 0.0676 & 0.86 \\ 0.0141 & 0.23 \\ -0.224 & -2.36 * * \\ 0.0188 & 0.26 \\ 0.0307 & 0.36 \\ 0.788 & 9.32 * * *\end{array}$

**Statistically significant at a $95 \%$ confidence level

$* * *$ Statistically significant at a $99 \%$ confidence level

In both runs, the change in estimated natural infiltration and the change in outdoor temperature are statistically significant and have the expected sign: an increase in radon is associated with tighter homes 
and lower outdoor temperature. The installation of continuous mechanical ventilation is also statistically significant (at a 95\% confidence level) in both runs, and suggests that radon levels are reduced when such ventilation is installed. The addition of non-continuous ventilation and dryer venting was not associated with a statistically significant change in radon level. For the addition of continuous ventilation caution is in order, since the data set contains only 21 homes that received such mechanical ventilation. Finally, both runs suggest that radon levels are reduced in mobile homes following weatherization (though this result is also based on only 25 mobile homes in the sample).

Of the other parameters, while some are statistically significant for the unscreened run, none are significant when the data are screened to remove very large changes in radon levels. Given that the second run screens out only eight cases, these parameters are not considered to be particularly well determined from this data set. Similar instability was observed in these (and other) parameters in a variety of models that were considered.

That measured changes in air tightness were found to be a statistically significant predictor of changes in radon levels - in addition to air leakage being a good predictor of pre-weatherization radon levels across homes - speaks to its likely primacy in affecting indoor radon levels. This is not to say that all air sealing activity by weatherization necessarily increases radon levels in homes: as noted above, below-grade sealing - and air-sealing that isolates living spaces from foundation spaces - could serve to reduce radon intrusion. However, the data suggest that on average, the overall effect of air sealing is a reduction in natural ventilation that results in a proportionately higher indoor radon concentration. In the majority of program homes with low pre-weatherization radon levels, this will result in only a slight increase in absolute radon levels, but in a minority of homes with elevated pre-existing radon, air-sealing will have a more significant impact on absolute radon level.

The failure of the analysis to identify ground covers and below-grade sealing as statistically significant weatherization-related factors that affect radon may speak to the fact that these are not typically implemented with radon mitigation in mind - and may also speak to the limited nature of the data at hand. For example, while data were available on whether there were attempts to isolate the foundation from the ground (either by adding a ground cover over bare earth or caulking cracks), these data did not provide details on exactly how much exposure there was or how well the measures were installed from a radon mitigation perspective (ground covers are typically employed by weatherization for moisture control). The variability of radon results due to non-weatherization factors, such as soil condition, also reduces the ability of the analysis to tease out statistical significance of weatherization measures. With a one-week sample it is plausible that radon changes due to environmental factors for which data were lacking (such as soil saturation and frost depth) may be greater than the potential impact of these weatherization measures.

\subsection{FORMALDEHYDE}

Formaldehyde is a common indoor contaminant. It is emitted by building materials and furnishings, especially those made of composite wood materials, as well as cigarettes and other forms of combustion. It is both an irritant and a carcinogen. Most existing standards and guidelines for formaldehyde are based on eye or respiratory tract irritation effects. A wide range of exposure limits have been proposed or adopted for formaldehyde. Current guidelines include $40 \mathrm{ppb}$ on an 8-hour average basis and $100 \mathrm{ppb}$ for 1-hour exposure in residential settings (Health Canada 2006), while the state of California has established "Reference Exposure Levels," intended to protect the most sensitive individuals from adverse health effects, of $7 \mathrm{ppb}$ for either an 8-hour average or chronic exposure, and $44 \mathrm{ppb}$ for 1-hour exposure (OEHHA 2008). Permissible levels are substantially higher in workplace environments, e.g. the OSHA workplace 8-hr time weighted average is set at $750 \mathrm{ppb}$. (OSHA 2011). 
Indoor formaldehyde levels were measured in approximately one in four study homes, based on a protocol of deploying a sampler in the first treatment and control group homes in each sampled geographic area of six to eight sites. ${ }^{26}$ The samplers were deployed on the lowest main living level of the home alongside the radon canister. Exposure times ranged from five to 11 days, with a median of eight days. Ninety percent of the tests were based on a seven- to nine-day exposure period.

\subsubsection{Pre-Weatherization Formaldehyde}

Formaldehyde levels measured prior to weatherization ranged from less than one to 72 parts per billion (ppb), with results for most homes falling below $30 \mathrm{ppb}$ (Fig. 19). The population-weighted estimate of the average indoor formaldehyde concentration in WAP single-family homes is $14 \pm 1 \mathrm{ppb}$, with mobile homes showing a somewhat higher average concentration than site-built homes, though the difference is not statistically significant (Table 12). These results are comparable to what others have found in older U.S. homes (e.g., Hun et al., 2010). In contrast, Offermann (2009) reported a median indoor formaldehyde concentration of $30 \mathrm{ppb}$ for 105 new California homes, with a range of four to $113 \mathrm{ppb}$.

Though cigarette smoke is a known source of formaldehyde, no statistically significant relationship was found between pre-weatherization formaldehyde levels and the presence of a smoker in the household. However, the relatively small sample of homes with formaldehyde results meant there were only 29 households with reported indoor smoking by the respondent for the analysis, which translates into low statistical power for detecting differences. Also, the occupant survey did not query about smoking habits of all household members.

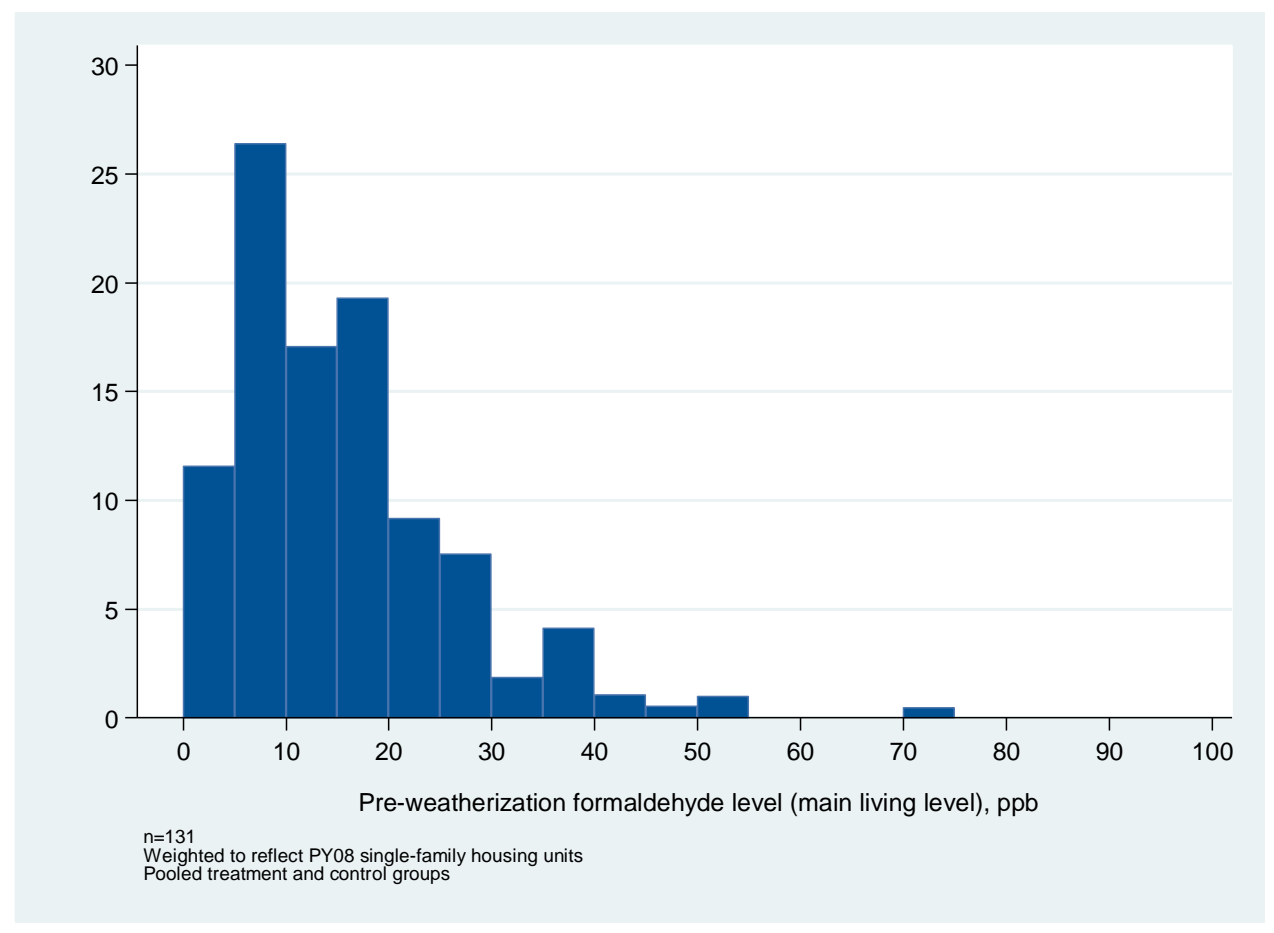

Fig. 19. Distribution of pre-weatherization formaldehyde level.

\footnotetext{
${ }^{26}$ Assay Technology 571-Aldehyde passive samplers were used, with analysis and results reporting by Galson Laboratories.
} 
Table 12. Pre-weatherization formaldehyde level.

\begin{tabular}{lccc}
\hline & $\mathbf{n}$ & $\begin{array}{c}\text { Percent below } \\
\text { detection limit } \\
(\mathbf{0 . 5} \mathbf{~ p p b )}\end{array}$ & Mean (ppb) \\
\hline Overall & 131 & $0 \%$ & $15 \pm 1$ \\
Site-built homes & 105 & $0 \%$ & $14 \pm 1$ \\
Mobile homes & 26 & $0 \%$ & $20 \pm 3$ \\
\hline
\end{tabular}

Results are for the first-floor of the home under closed-home conditions.

Results are weighted to reflect the population of single-family homes treated by the program in

PY08.

Treatment and control groups are pooled.

\pm values are approximate $90 \%$ confidence intervals.

\subsubsection{Change in Formaldehyde Levels}

Table 13 summarizes the weighted-average change in formaldehyde levels associated with weatherization for the 119 homes with pre- and post-weatherization test results, and Fig. 20 shows scatter plots of preversus post-weatherization measured levels. Formaldehyde concentrations increased in both the treatment and control groups following weatherization, though by somewhat more in the former. This leads to an estimated net impact of $1.6 \pm 1.1 \mathrm{ppb}$ following weatherization.

The estimated $4.2 \pm 5.2 \mathrm{ppb}$ change in formaldehyde concentration in mobile homes is not statistically significant, but the wide confidence interval leaves open the possibility that a larger sample of mobile homes might reveal a statistically_ and perhaps meaningfully — significant impact of weatherization on formaldehyde in this housing type. ${ }^{27}$ However, note that the small number of mobile homes in this analysis was not well balanced geographically, leaving open the possibility that the results below could be confounded by weather and other non-program differences between the treatment and control groups.

Table 13. Change in formaldehyde level.

\begin{tabular}{|c|c|c|c|c|c|c|}
\hline & \multirow[b]{2}{*}{$\mathbf{N}$} & \multicolumn{2}{|c|}{$\begin{array}{c}\text { Mean Pre-Wx } \\
\text { formaldehyde } \\
\text { level (ppb) }\end{array}$} & \multicolumn{3}{|c|}{ Mean change in formaldehyde level (ppb) } \\
\hline & & $\begin{array}{c}\text { Trt } \\
(n=63)\end{array}$ & $\begin{array}{c}\text { Cntrl } \\
(n=56)\end{array}$ & $\begin{array}{c}\text { Treatment } \\
\text { Group }\end{array}$ & $\begin{array}{c}\text { Control } \\
\text { Group }\end{array}$ & $\begin{array}{c}\text { Net } \\
\text { (treatment - } \\
\text { control) } \\
\end{array}$ \\
\hline Overall & 119 & 15 & 16 & $+3.5 \pm 1.6$ & $+1.9 \pm 1.5$ & $+1.6 \pm 1.1$ \\
\hline Site-built homes & 96 & 13 & 16 & $+3.1 \pm 1.8$ & $+2.3 \pm 1.5$ & $+0.8 \pm 2.2$ \\
\hline Mobile homes & 23 & 22 & 17 & $+4.8 \pm 3.0$ & $+0.6 \pm 4.0$ & $+4.2 \pm 5.1$ \\
\hline
\end{tabular}

Results are for the first-floor of the home under closed-home conditions for sites with both pre- and post-weatherization test results, and are weighted to reflect the population of single-family homes treated by the program in PY08.

\pm values are approximate $90 \%$ confidence intervals.

\footnotetext{
${ }^{27}$ There were only 10 treatment mobile homes and 13 control mobile homes in this analysis. At these sample sizes, one would only expect to be able to reliably detect a doubling in formaldehyde level (power $=0.85$ ).
} 


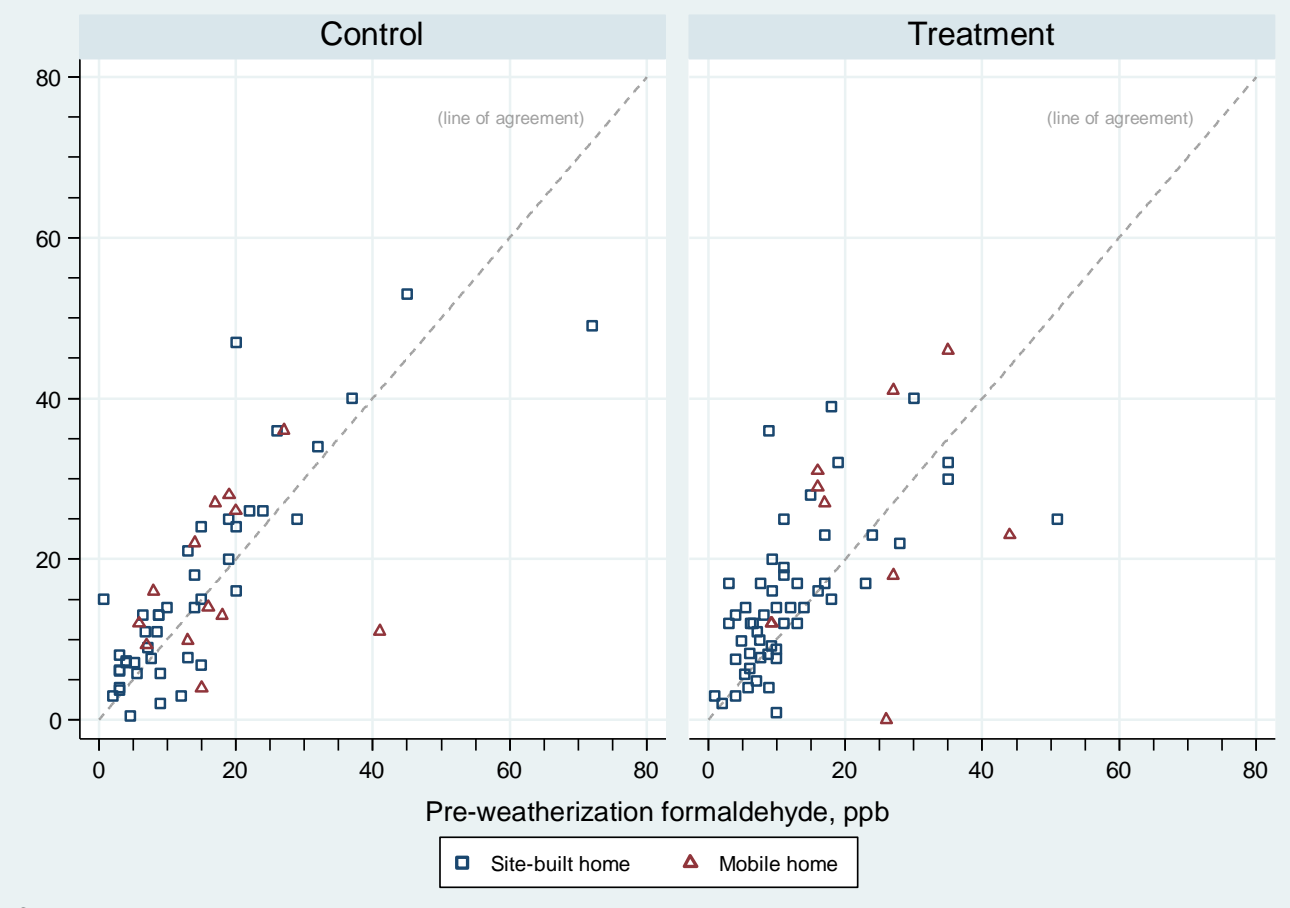

Control $n=56$; Treatment $n=63$

Fig. 20. Pre- versus post-weatherization indoor formaldehyde level.

Formaldehyde levels are known to vary with temperature and humidity: higher temperatures and higher humidity increase formaldehyde emissions. Data from the temperature and humidity data loggers in the study homes confirms a fairly strong relationship between changes in formaldehyde levels and changes indoor humidity, as Fig. 21 shows. The relationship with temperature changes is much weaker.

When averaged across the sites in the formaldehyde sub-sample, the treatment and control groups had comparable indoor conditions prior to weatherization (Table 14). Following weatherization, relative humidity increased more in the treatment group than in the comparison group, though the difference was not statistically significant.

As documented later in this report, the study data show that weatherization is generally associated with a small increase in relative humidity. It is thus plausible that the observed net increase in formaldehyde levels is due to a combination of lower air-exchange rates and increased formaldehyde emission rates from slightly higher indoor humidity. 


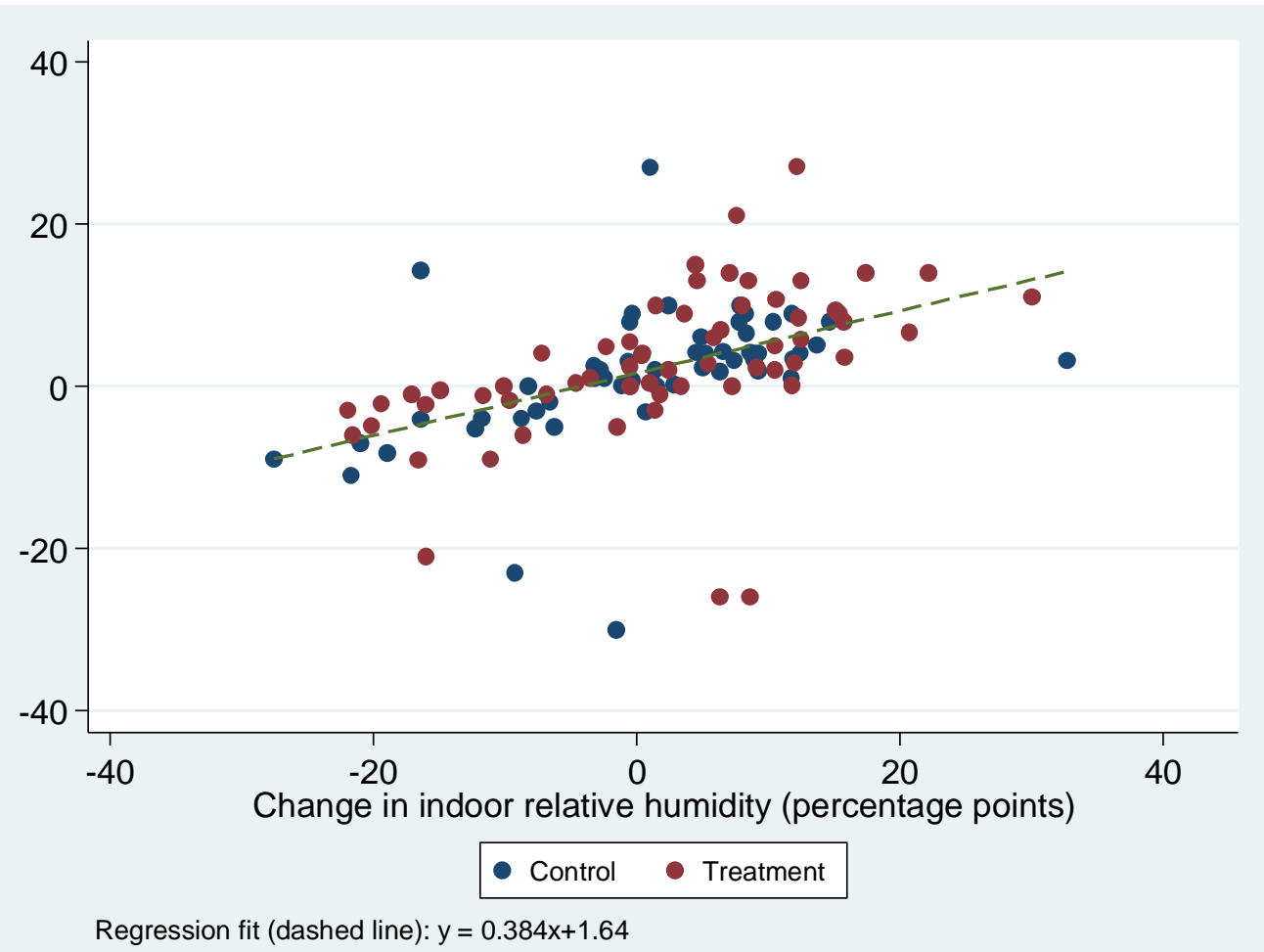

Fig. 21. Change in formaldehyde level vs. change in relative humidity.

Table 14. Indoor temperature and relative humidity for formaldehyde sub-sample.

\begin{tabular}{lrccc}
\hline & & $\begin{array}{c}\text { Pre- } \\
\text { Weatherization }\end{array}$ & $\begin{array}{c}\text { Post- } \\
\text { Weatherization }\end{array}$ & $\begin{array}{c}\text { Change } \\
\text { (Post - Pre) }\end{array}$ \\
\hline Temperature (F) & Treatment & $71.0 \pm 0.7$ & $70.6 \pm 0.8$ & $-0.3 \pm 0.4$ \\
& Control & $71.4 \pm 0.9$ & $71.7 \pm 1.0$ & $0.3 \pm 0.3$ \\
Relative humidity & Treatment & $34.5 \pm 2.6$ & $36.7 \pm 2.6$ & $+2.2 \pm 2.4$ \\
$(\%)$ & Control & $32.6 \pm 2.5$ & $33.6 \pm 2.7$ & $+0.9 \pm 2.5$ \\
\hline
\end{tabular}

For sites with both pre- and post-weatherization formaldehyde test results: treatment $n=63$; control $n=56$. Weighted to reflect the population of single-family homes treated by the program in PY08.

\pm values are approximate $90 \%$ confidence intervals.

\subsection{TEMPERATURE}

Indoor temperature is arguably the single most important driver of perceived comfort. Although ASHRAE has defined a heating season comfort range from about $68 \mathrm{~F}$ to 77F (ASHRAE 2010a), this is geared for building and mechanical system design purposes, and individual preferences vary widely. Indoor temperature is of interest for weatherization because increased insulation and reduced air leakage may warm spaces that previously could not be maintained at the desired temperature by occupants. In a related vein, it is likely that some low-income households deliberately maintain a lower-than-desired indoor temperature in order to reduce heating bills. It has thus been argued that occupants may "take back" some of the energy savings from weatherization by increasing the thermostat set-point.

To study indoor temperature, technicians hung a temperature (and relative humidity) data logger from the main thermostat to take a snapshot of indoor conditions every 10 minutes over the duration of the study 
period. ${ }^{28}$ For the small number of homes without a functional central thermostat, the logger was deployed in a comparable central location. Technicians also recorded whether the home had a programmable thermostat, and if so, whether the thermostat was turned off, running a program, or was in "Hold" mode at the time of each site visit. The analysis presented here focuses on results for the majority of sites that were instrumented during the heating season, and omits the small number of sites in southern states that were monitored during the summer.

To analyze the temperature data, the time trace for each site was visually scanned first to identify and remove anomalous periods such as prolonged (and deep) temperature setbacks suggestive of a period when the home was unoccupied. This step affected less than one percent of the data.

Data were also dropped for days where the daily average outdoor temperature (from nearby weather stations) was 55F or higher. The purpose of this step, which removed about three percent of the data, was to restrict the analysis to time periods when the home was likely to be in a closed, space-heating condition. In addition, data were dropped from the first week following completion of weatherization work.

Finally, to minimize the impact of weather on pre/post weatherization differences, daily indoor temperature was regressed against outdoor temperature for each site (separately for the pre- and postweatherization periods), and indoor temperatures were adjusted to typical winter-time conditions. ${ }^{29}$ Overall, these adjustments were not large, but some sites had a noticeably strong relationship between the two. And although pre- and post-weatherization outdoor temperatures averaged out to the same value (29F) across the study sample, outdoor temperatures varied by as much as $30 \mathrm{~F}$ in the two periods for some sites.

After dropping sites with less than a week of temperature data, there was usable pre- and postweatherization temperature data for 478 and 462 sites, respectively, with 460 sites having both pre- and post-weatherization data. Note that the analysis presented here does not include the small number of homes in the South that were monitored in the summer.

\subsubsection{Pre-Weatherization Indoor Temperature}

The data indicate that the average program home has a winter indoor temperature of $70.3 \pm 0.4 \mathrm{~F}$ prior to weatherization, though the distribution ranges from less than $60 \mathrm{~F}$ to more than $80 \mathrm{~F} \mathrm{(Fig.} \mathrm{22).}$

The data reveal clear evidence of routine temperature setback among some homes. Based on an algorithm that examines average hourly temperatures, it was estimated that $24 \pm 5$ percent of homes practice some form of setback during the heating season. ${ }^{30}$ Indoor temperature in these homes averages $3.0 \pm 0.7 \mathrm{~F}$ less than in homes that do not exhibit evidence of regular setback.

\footnotetext{
${ }^{28}$ Hobo U-10-003 loggers were used. These have a listed temperature accuracy of about $1 \mathrm{~F}$ under typical indoor conditions.

${ }^{29}$ Specifically, indoor temperatures were normalized to the 10-year average December-February temperature for the weather station associated with each site.

${ }^{30}$ The algorithm flagged a site as practicing thermostat setback if the average temperature for any hour of the day (across all days) differed from the overall average temperature by more than $2 \mathrm{~F}$.
} 


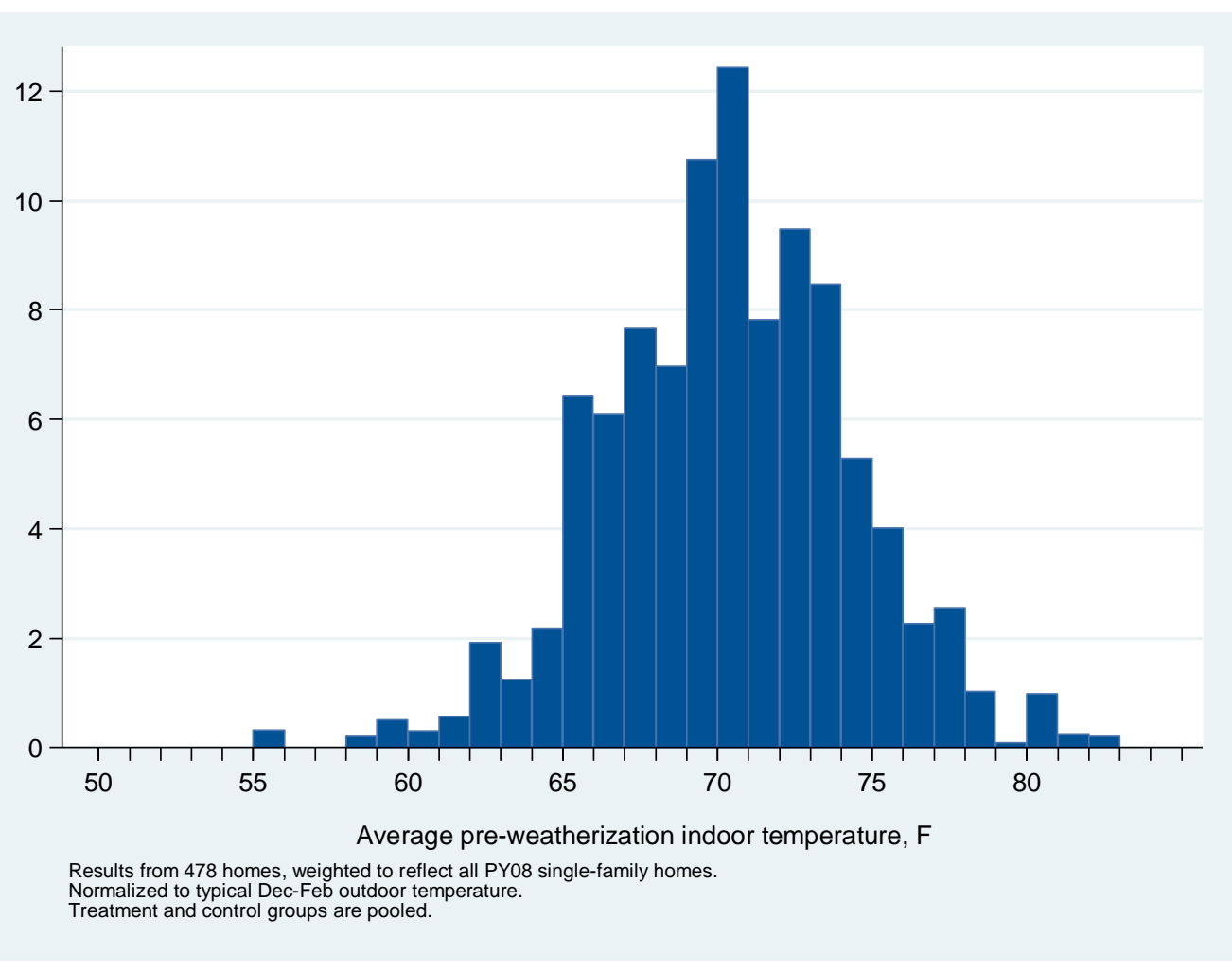

Fig. 22. Pre-weatherization indoor temperature (winter).

Homes with existing programmable thermostats are significantly more likely to show empirical evidence of temperature setback. These homes also have indoor temperatures that average $1.5 \pm 0.5 \mathrm{~F}$ lower than homes with a manual (or no) thermostat (Table 15).

Table 15. Indoor temperature and temperature setback, by type of thermostat.

\begin{tabular}{lcccc}
\hline & \multicolumn{2}{c}{$\begin{array}{c}\text { Evidence of setback } \\
\text { (\% of homes) }\end{array}$} & \multicolumn{2}{c}{$\begin{array}{c}\text { Mean indoor } \\
\text { temperature (F) }\end{array}$} \\
\hline Overall & 24 & \pm 5 & 70.3 & \pm 0.5 \\
Manual (or no) thermostat $(74 \pm 4 \%)$ & 19 & \pm 4 & 70.8 & \pm 0.4 \\
Programmable thermostat $(26 \pm 4 \%)$ & 34 & \pm 9 & 69.2 & \pm 0.5 \\
\hline
\end{tabular}

Weighted to reflect all single-family homes in PY08.

Evidence of setback based on $\geq 2 \mathrm{~F}$ deviation in average hourly temperature compared to average over all hours.

Mean indoor temperature normalized to Dec-Feb average outdoor temperature.

\pm values are approximate $90 \%$ confidence intervals.

Also, a linear regression analysis of explanatory factors for indoor temperature was undertaken using site data and the occupant survey (Table 16).

The results reveal several factors that are significantly correlated with higher or lower indoor temperatures prior to weatherization (in roughly descending order of effect size and statistical significance):

- Households with Black/African American respondents average about 4F higher indoor temperatures than do other households. 
- Each decade of age (for the oldest household member) is associated with about a $0.5 \mathrm{~F}$ increase in indoor temperature.

- Temperatures in mobile homes are about $2 \mathrm{~F}$ higher than in site-built homes.

- Respondents with a high school diploma or college degree average 1 to $2 \mathrm{~F}$ lower indoor temperatures.

- Homes with children average about $1 \mathrm{~F}$ higher than other homes.

- Homes with basements average about $1 \mathrm{~F}$ warmer than other homes.

- Homes with oil heat average about $1 \mathrm{~F}$ cooler than other homes.

Table 16. Model of pre-weatherization indoor temperature (pooled treatment and control groups).

\begin{tabular}{|c|c|c|c|c|}
\hline $\begin{array}{c}\text { Dependent variable: pre-weatherization indoor } \\
\text { temperature }^{\dagger}\end{array}$ & $\begin{array}{l}\text { Weighted } \\
\text { incidence in } \\
\text { sample }\end{array}$ & $\begin{array}{l}\text { Temperature } \\
\text { effect }\end{array}$ & t-statistic & $\begin{array}{l}\text { Signifi - } \\
\text { cance } \\
\text { level }\end{array}$ \\
\hline Renter & $7 \%$ & +0.60 & 0.81 & \\
\hline Respondent Black/African American & $15 \%$ & +3.87 & 6.28 & $* * *$ \\
\hline Respondent Hispanic & $10 \%$ & -0.52 & -0.81 & \\
\hline $\begin{array}{l}\text { Decades of age for oldest household member (range: 2- } \\
\text { 8) }\end{array}$ & & +0.45 & 3.35 & $* * *$ \\
\hline Child in home & $38 \%$ & +1.37 & 4.00 & $* * *$ \\
\hline High school diploma & $35 \%$ & -1.22 & -2.18 & $* *$ \\
\hline Some college or college degree & $42 \%$ & -1.86 & -3.53 & $* * *$ \\
\hline Mobile home & $26 \%$ & +1.86 & 2.60 & $* * *$ \\
\hline Site-built attached home & $6 \%$ & +1.06 & 2.27 & $* *$ \\
\hline Multi-story home & $28 \%$ & -0.79 & -1.72 & $*$ \\
\hline Home has a $250+\mathrm{ft}^{2}$ basement & $43 \%$ & +1.16 & 2.34 & $* *$ \\
\hline Home has a $250+\mathrm{ft}^{2}$ crawlspace & $30 \%$ & +0.32 & 0.67 & \\
\hline Home has a $250+\mathrm{ft}^{2}$ slab & $11 \%$ & +1.05 & 1.65 & \\
\hline Electric heat & $19 \%$ & +0.16 & 0.24 & \\
\hline Oil heat & $15 \%$ & -1.27 & -2.17 & $* *$ \\
\hline Wood/other heat & $3 \%$ & +0.22 & 0.20 & \\
\hline Model constant & & 67.23 & 61.40 & \\
\hline
\end{tabular}

Regression statistics: $\mathrm{n}=438$; adjusted $\mathrm{r}^{2}=0.200 ;$ F statistic $=14.79$

${ }^{\dagger}$ Normalized to Dec-Feb typical outdoor temperature for site.

*Statistically significant at a 90 percent confidence level

**Statistically significant at a 95 percent confidence level

*** Statistically significant at a 99 percent confidence level

\subsubsection{Change in Indoor Temperature}

On the whole, indoor temperatures in the weeks following weatherization tracked pre-weatherization temperatures fairly closely (Fig. 23). Temperatures in the treatment group averaged 0.14F higher following weatherization, and temperatures in the control group declined by $0.13 \mathrm{~F}$. The net difference between the two $(0.3 \pm 0.2 \mathrm{~F})$ is small but statistically significant (at about a 90 percent confidence level), and suggests an average temperature increase among weatherized homes of about $0.5 \mathrm{~F}$ or less. 


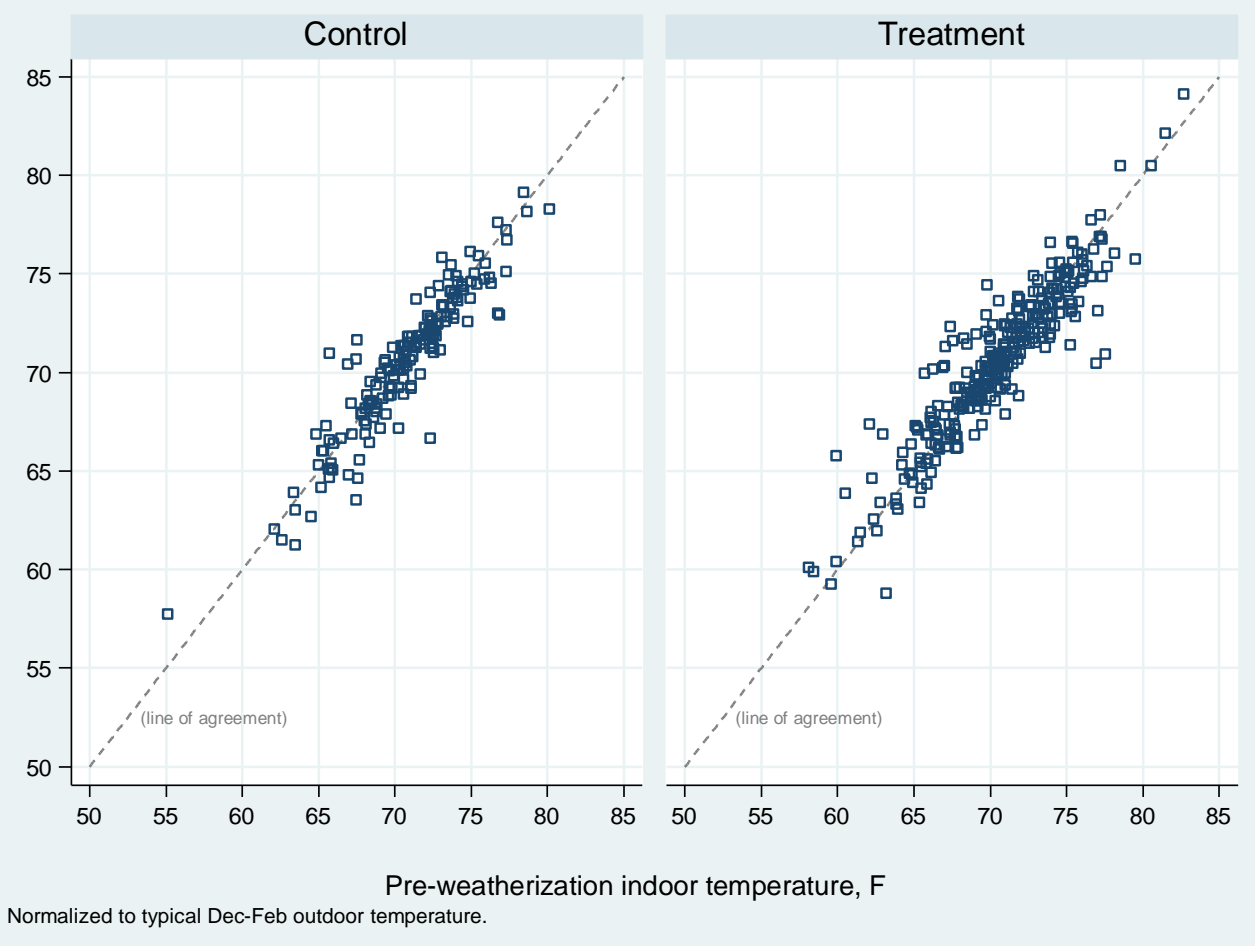

Fig. 23. Pre- versus post-weatherization indoor temperature.

As Fig. 24 shows, there is some evidence of a "regression to the mean" effect with indoor temperature: that is, indoor temperature tended to increase among households on the low end of pre-weatherization temperature, and decrease among households on the high end. This suggests that the more extreme recorded temperatures in both groups tended to be temporary phenomena.

About one in ten treatment-group homes received a setback thermostat as part of weatherization, a proportion that matches the national incidence rate among single-family homes for this measure in PY08. Compared to other households in the study, there was no significant difference in the proportion of these households that began to practice thermostat setback following weatherization (Table 17), though the small number of homes involved makes the comparison imprecise. 


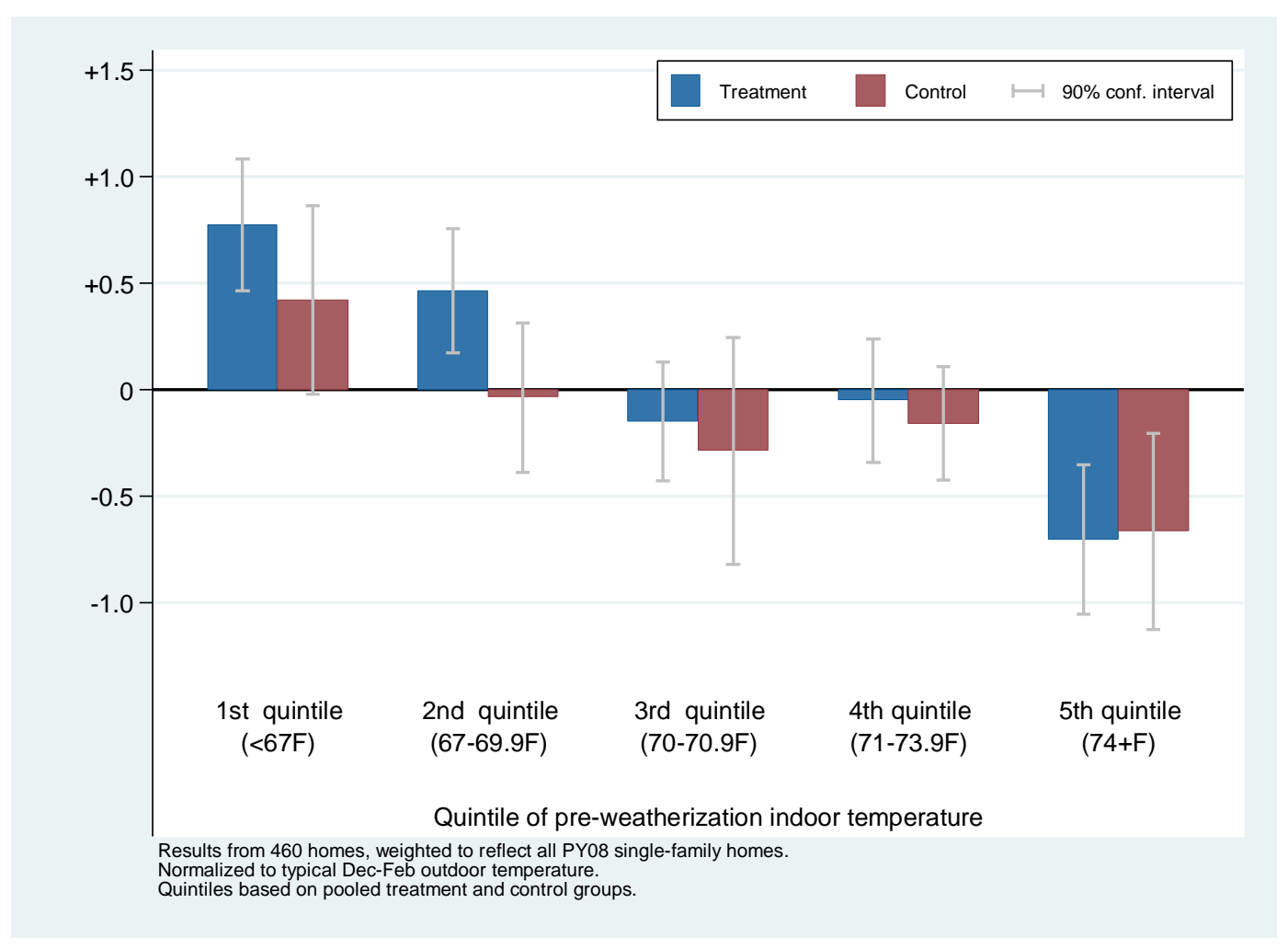

Fig. 24. Change in indoor temperature by pre-weatherization temperature quintile.

Table 17. Pre- and post-weatherization evidence of temperature setback, by group and (for treatment homes) whether a new programmable thermostat was installed.

\begin{tabular}{ccccc}
\hline \multirow{2}{*}{ Evidence of setback? } & \multicolumn{2}{c}{ Treatment group } & Control \\
\cline { 3 - 5 } Pre-Weatherization & Post-Weatherization & $\begin{array}{c}\text { Thermostat not } \\
\text { replaced } \\
\text { (n=256) }\end{array}$ & $\begin{array}{c}\text { Received new } \\
\text { programmable thermostat } \\
(\mathbf{n = 3 5 )}\end{array}$ & $\begin{array}{c}\text { group } \\
(\mathbf{n = 1 6 8})\end{array}$ \\
\hline No & No & $68 \%$ & $75 \%$ & $69 \%$ \\
No & Yes & $7 \%$ & $9 \%$ & $7 \%$ \\
Yes & No & $4 \%$ & $1 \%$ & $3 \%$ \\
Yes & Yes & $21 \%$ & $15 \%$ & $21 \%$ \\
& & $100 \%$ & $100 \%$ & $100 \%$ \\
\hline
\end{tabular}

Weighted to reflect all single-family homes in PY08.

Evidence of setback based on $\geq 2 \mathrm{~F}$ deviation in average hourly temperature compared to average over all hours.

\subsection{INDOOR HUMIDITY AND MOISTURE}

Indoor humidity is an important consideration for comfort, health and building preservation. Low relative humidity tends to dry mucous membranes, leads to dry skin and can create respiratory irritation and static electricity problems. ASHRAE (ASHRAE 2010a) recommends an upper limit of about 80 percent relative humidity at 70F - which is rarely achieved in indoor environments - but general relative humidity above even 50 percent promotes the growth of allergens such as dust mites, and may lead to mold growth in areas of the home where the temperature is cooler. 
Humidity can be expressed in different ways, and each is useful in its own right. The study focus is on:

- Relative humidity - the amount of moisture in the air relative to the maximum that can be held in the air at a given temperature. Cold air can hold less moisture than warm air, and relative humidity will be higher at a cooler temperature compared to the same air at a warmer temperature.

- Dew-point temperature - the temperature at which moisture begins to condense from air. The higher the humidity, the higher the dew-point temperature. Dew-point temperature is most relevant for assessing the potential for condensation on cold surfaces, primarily windows.

- Humidity ratio - a measure of the absolute amount of moisture in a given amount of air, expressed as the ratio of the mass of moisture in a given mass of dry air.

The data loggers used to record indoor humidity for the study (described above under "Indoor Temperature") directly sense and record relative humidity; when combined with the concurrently logged indoor temperature, dew-point temperature and humidity ratio can be readily calculated. An important limitation of the data is that the data loggers that were deployed have a lower measurement limit of 15 percent relative humidity: overall, about 10 percent of the data collected was at or below this limit, but for some sites, this affected the majority of the data collected.

Indoor humidity is typically correlated with outdoor humidity, and outdoor humidity is strongly dependent on outdoor temperature. Because cold outdoor air can hold far less moisture than warm air, indoor humidity tends to decrease in cold weather and increase in warmer weather. To control for this, measured indoor humidity was normalized to typical December-February outdoor conditions for each site. ${ }^{31}$ Note that the analysis presented here omits the small number of sites that were monitored in southern states during the summer.

\subsubsection{Pre-Weatherization Indoor Humidity}

The study data suggest that the average program home has a winter indoor relative humidity of $33 \pm 2$ percent, though this estimate is likely somewhat high due to the fact that some homes in the study had measured relative humidity at or below the 15 percent lower limit for the data loggers (Fig. 25). On the whole, weatherization homes tend to be on the dry side: $44 \pm 5$ percent of homes have relative humidity below 30 percent, but only $6 \pm 4$ percent have relative humidity over 50 percent.

Dew-point temperature averages $39 \pm 1 \mathrm{~F}$ in program homes (subject to the same potential bias issue noted above). Winter dew-point temperatures were highest among study homes in the warmest climate (California), but for homes in climates with an average December-February outdoor temperature of less than $40 \mathrm{~F}$, there is a wide range of indoor dew-point temperatures (Fig. 26).

\footnotetext{
${ }^{31}$ Specifically, the indoor humidity ratio was regressed against the outdoor humidity ratio, and then the observed average indoor humidity ratio was adjusted to the 10 -year average December-February outdoor humidity ratio for each location.
} 


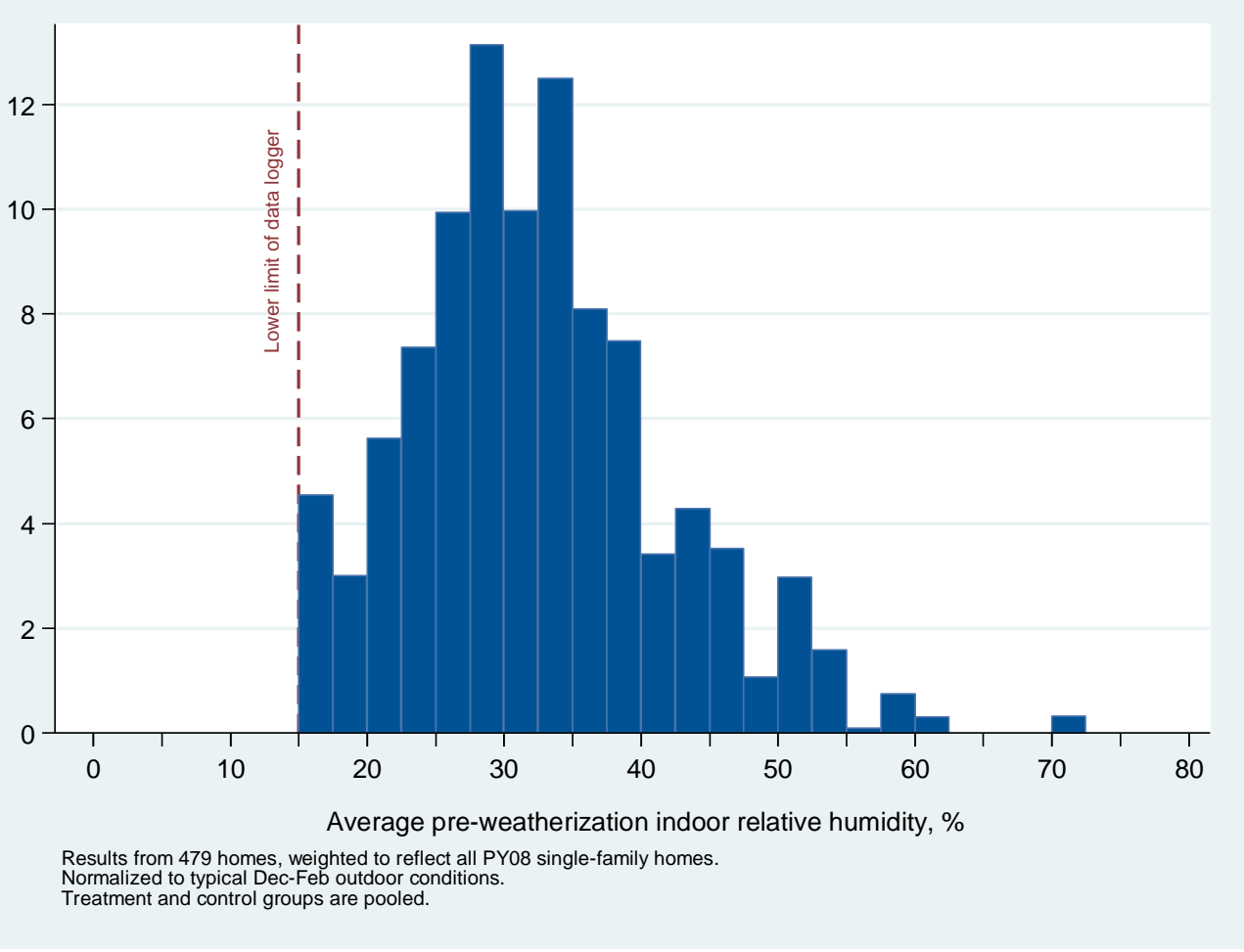

Fig. 25. Pre-weatherization indoor relative humidity (winter).

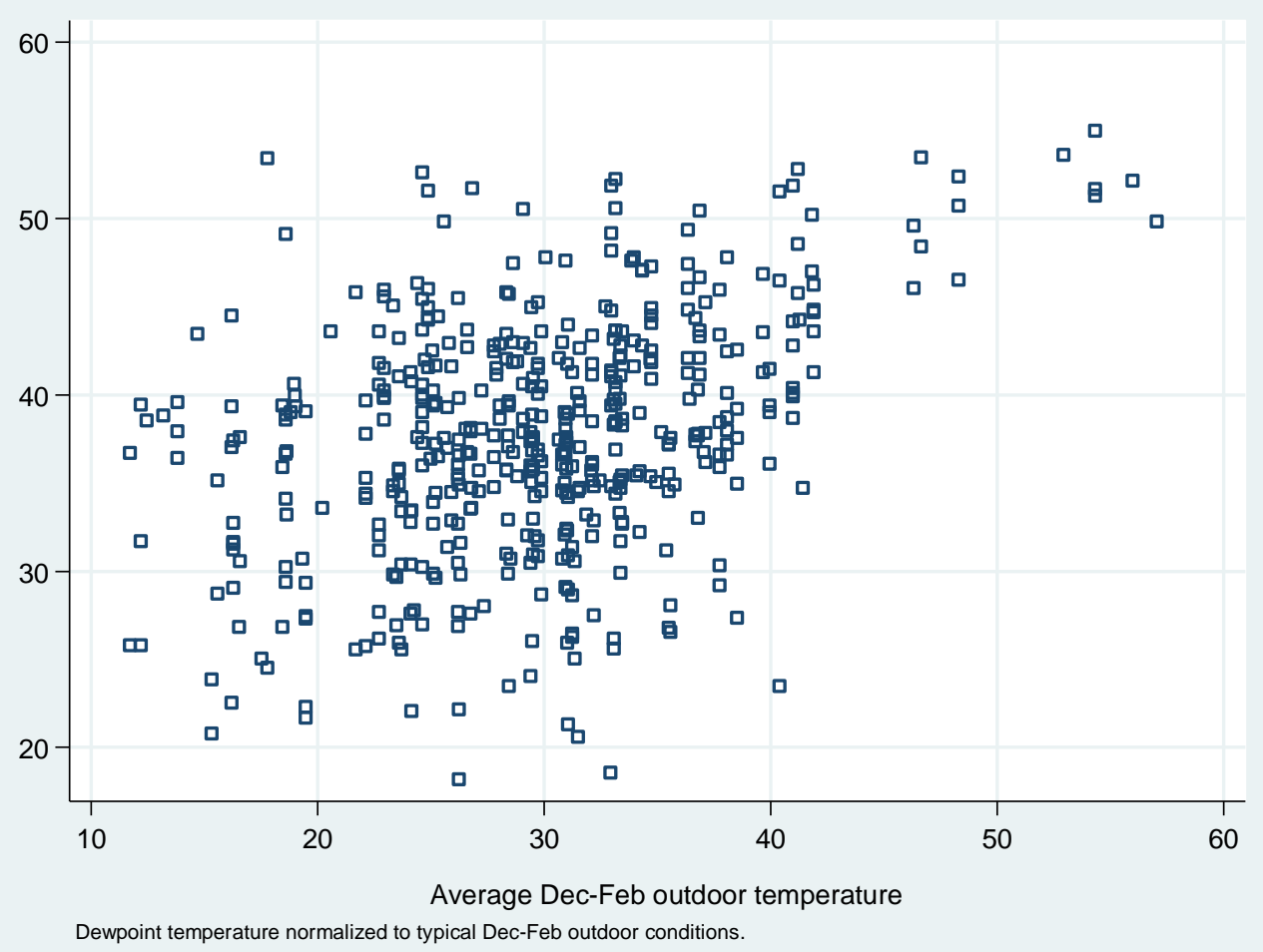

Fig. 26. Pre-weatherization indoor dew-point versus average winter outdoor temperature. 


\subsubsection{Change in indoor humidity}

Post-weatherization indoor humidity tended to track pre-weatherization humidity (Fig. 27), but there were some small but statistically significant average changes, as Table 18 shows. Both groups showed slight declines in indoor humidity from the pre- to post-weatherization period, signaling a general drying trend over the course of the heating season. But the control group declined more than the treatment group, and the net effect suggests a slight $(1.1 \pm 0.6 \%)$ increase in relative humidity associated with weatherization.

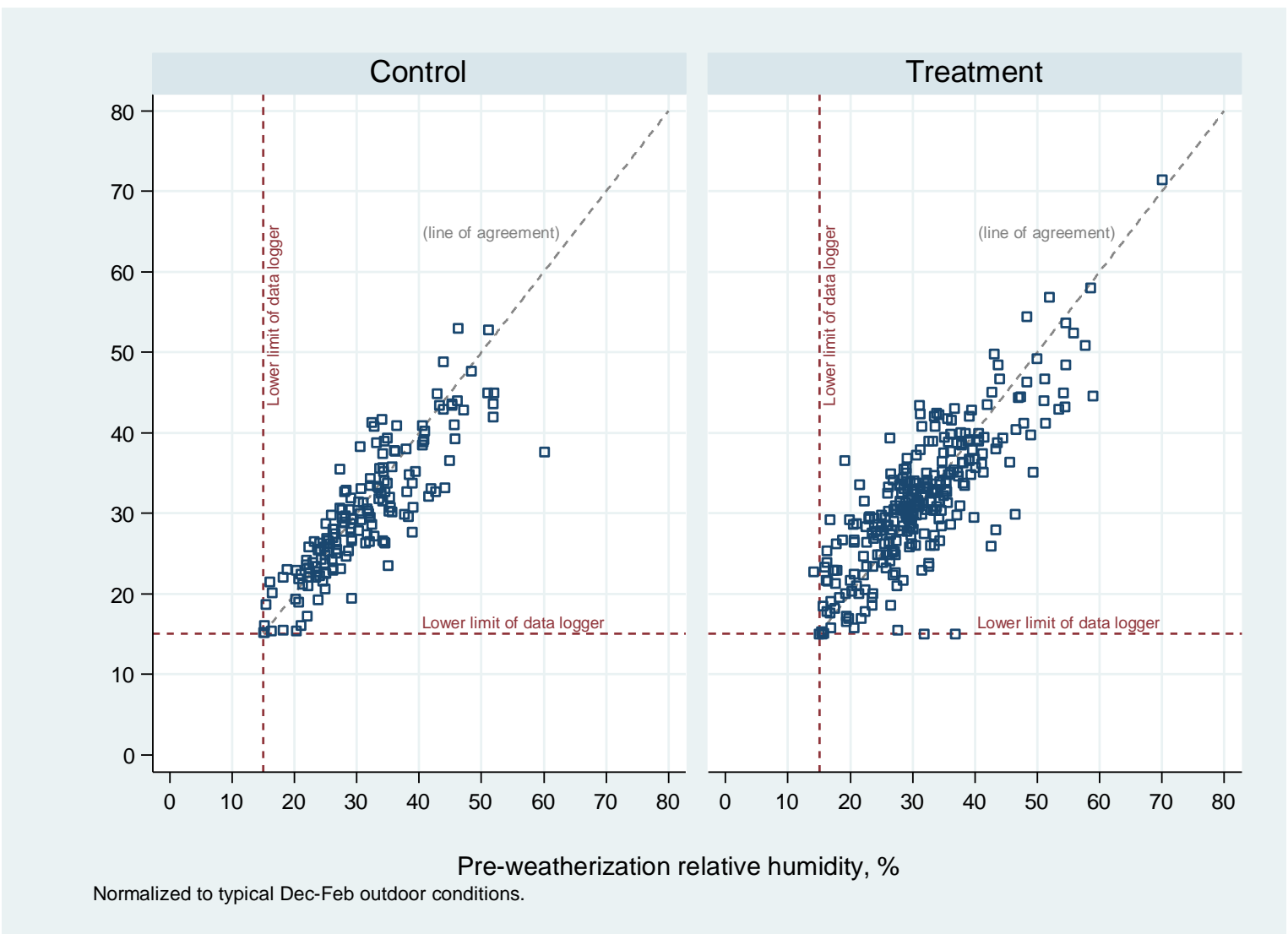

Fig. 27. Pre- versus post-weatherization indoor relative humidity.

Table 18. Change in indoor humidity from pre- to post-weatherization.

\begin{tabular}{lcccccc}
\hline & \multicolumn{2}{c}{$\begin{array}{c}\text { Treatment Group } \\
(\mathbf{n = 2 9 5})\end{array}$} & \multicolumn{2}{c}{$\begin{array}{c}\text { Control Group } \\
(\mathbf{n = 1 7 5})\end{array}$} & \multicolumn{2}{c}{$\begin{array}{c}\text { Net Effect } \\
\text { (Treatment - Control) }\end{array}$} \\
\hline Relative humidity (\%) & -0.3 & \pm 0.9 & -1.4 & \pm 0.9 & +1.1 & \pm 0.6 \\
Dew-point temperature (F) & +0.1 & \pm 0.7 & -1.1 & \pm 0.5 & +1.3 & \pm 0.4 \\
Humidity ratio (grains/lb.) & +0.1 & \pm 0.7 & -1.1 & \pm 0.6 & +1.3 & \pm 0.4 \\
\hline
\end{tabular}

Weighted to reflect all single-family homes in PY08.

Normalized to average Dec-Feb outdoor conditions.

\pm values are approximate $90 \%$ confidence intervals. 


\subsubsection{Moisture Observations}

In addition to the quantitative humidity measurements, moisture was evaluated qualitatively through visual observation. In both foundations and above-ground portions of the home, technicians were asked to identify observed musty smells, water stains, and mold. In foundation spaces, technicians were also asked to record standing water, and in above-ground spaces, technicians were asked to record observed window condensation. For water stains and mold, technicians were asked to specify whether the damaged area was less than two square feet, between two and 32 square feet, or greater than 32 square feet. For the other moisture problems, technicians were asked only to identify whether the problem was evident.

Because these data are subjective, there are limits to how much the results can be extrapolated to the entire weatherization population. For example, some technicians reported substantially more problems than others, with some technicians either not looking for moisture problems as thoroughly or choosing to not record very minor occurrences. As a result, the reporting here does not include estimates of how many locations each type of moisture issue was found within a home. However, estimates of how many homes had each type of issue are reported, as well as estimates of the severity of water staining and mold.

\section{Identified Moisture Problems}

Figure 28 shows identified moisture problems pre- and post-weatherization for foundations (left panel) and above grade (right panel). The categories identifying a specific moisture issue refer to cases where that was the only issue identified. These are weighted to reflect all PY2008 homes.

Unlike other IAQ issues in this report, moisture problems were not recorded based on measurement but rather observation. As such, the results are subject to technicians' rigor in inspecting the home and their decisions as to whether or not a condition qualified as a "problem." Therefore, recorded moisture issues may not accurately reflect the actual incidence of each issue. In these graphs, no attempt was made to adjust for technician rigor in identifying moisture issues. The figures are intended instead to illustrate which types of problems were more common than others. Because no attempt was made to adjust for technician, differences between pre- and post-weatherization should not be the focus but rather how the different issues compare.

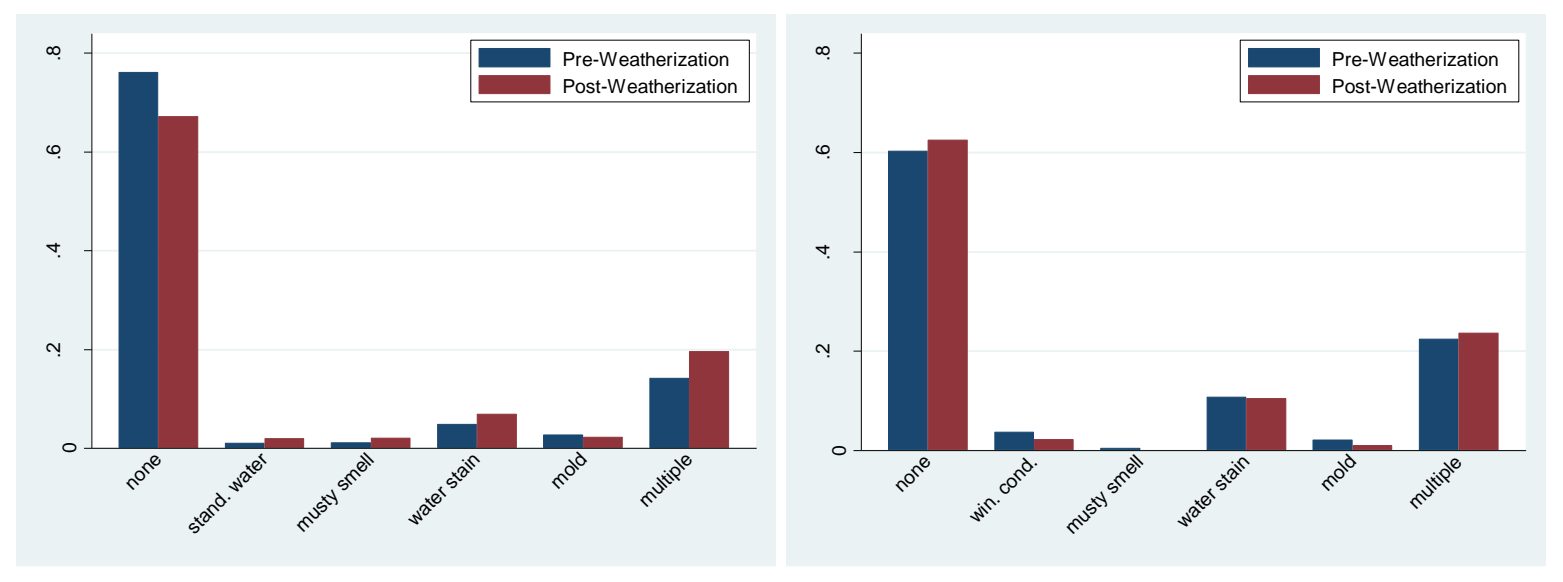

Fig. 28. Frequency of recorded moisture problems for foundations (left) and above grade (right).

These graphs show that about 65-75 percent of foundations and 60 percent of above-grade sections did not have any moisture issues identified by technicians. About 15-20 percent of foundations had multiple issues identified, whereas about a quarter of above-grade sections had multiple issues. In both foundation 
and above-grade spaces, water stains were the most prevalent issue when only one problem was noted. Musty smells were rarely noted as the only issue in above-grade spaces.

Figures 29 and 30 show observations of moisture problems for homes where the same technician evaluated the home both pre- and post-weatherization. This screening should mitigate the non-uniformity of technician observations. The results are weighted to reflect all PY2008 homes. The first figure shows foundation moisture problems, separated by control and treatment homes, and the second figure shows the corresponding graphs for above-grade moisture problems.

For foundations, there was an apparent increase in moisture problem observations following weatherization except for musty smells, which remained about constant, and mold, which showed a decrease in treatment homes. Compared to control homes, there appears to be a net increase in standing water and a net reduction in mold in treatment homes. However, these increases are not statistically significant. In foundations, water stains were the most commonly observed problem. No moisture problems were observed in more than 30 percent of foundations.

Problems were observed more frequently in above-grade spaces. All problems were observed less frequently after weatherization than before weatherization for both control and treatment groups. There were no statistically significant net changes when comparing control homes to treatment homes. Water stains were again the most frequently observed moisture problem in above-grade spaces. Window condensation was next highest in above-grade spaces, and was observed at a similar rate as water stains in control homes. Musty smells were the least frequently observed problem. No moisture problems were observed in more than 35 percent of above-grade spaces.

Finally, Fig. 31 shows the severity of water staining and mold problems recorded pre-weatherization for foundations and above-ground spaces for those homes where such problems were identified. The values shown are for the largest problem area of the home. Other than water stains in foundation spaces, the majority of homes with identified moisture problems had less than two square feet of moisture damage in the largest identified problem area. For foundations, the majority of homes with identified moisture problems had between two and 32 square feet of water staining. Few homes had more than 32 square feet of either water stains or mold above grade. Mold of greater than two square feet was found above-grade in only about 25 percent of the homes with identified above-grade mold.
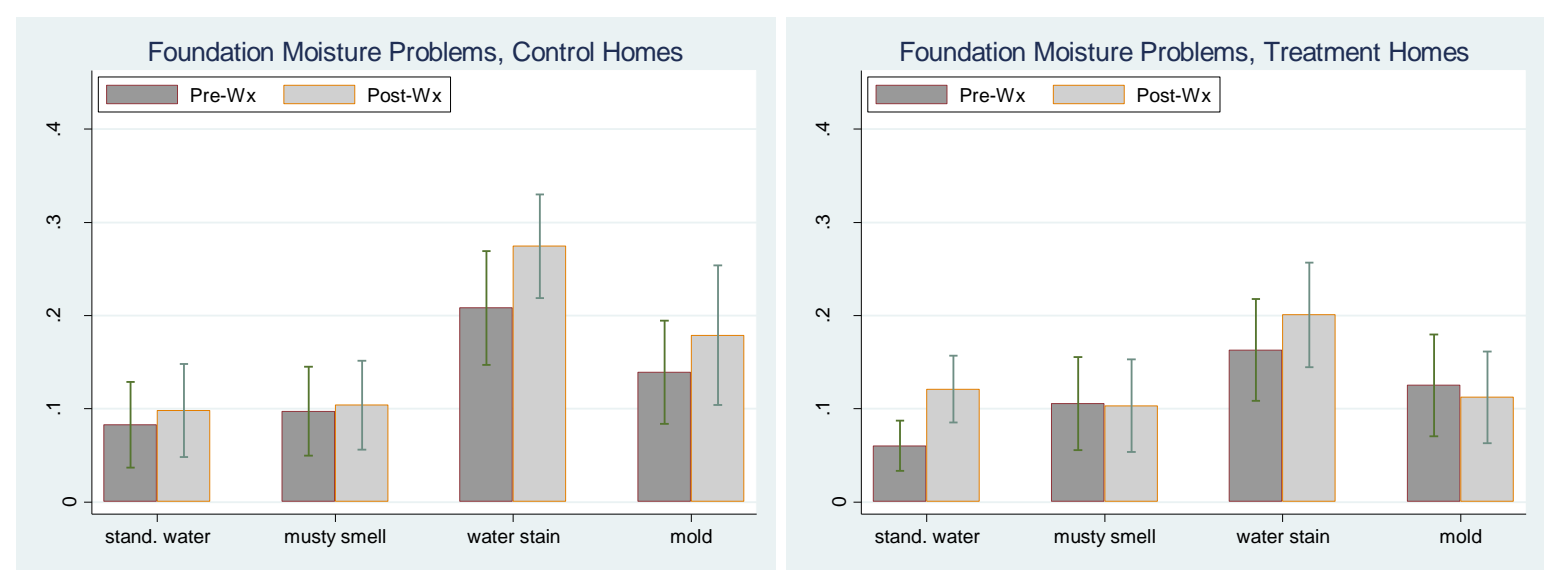

Fig. 29. Incidence of recorded foundation moisture problems for sites with the same technician pre- and post-weatherization (with $90 \%$ confidence intervals). 

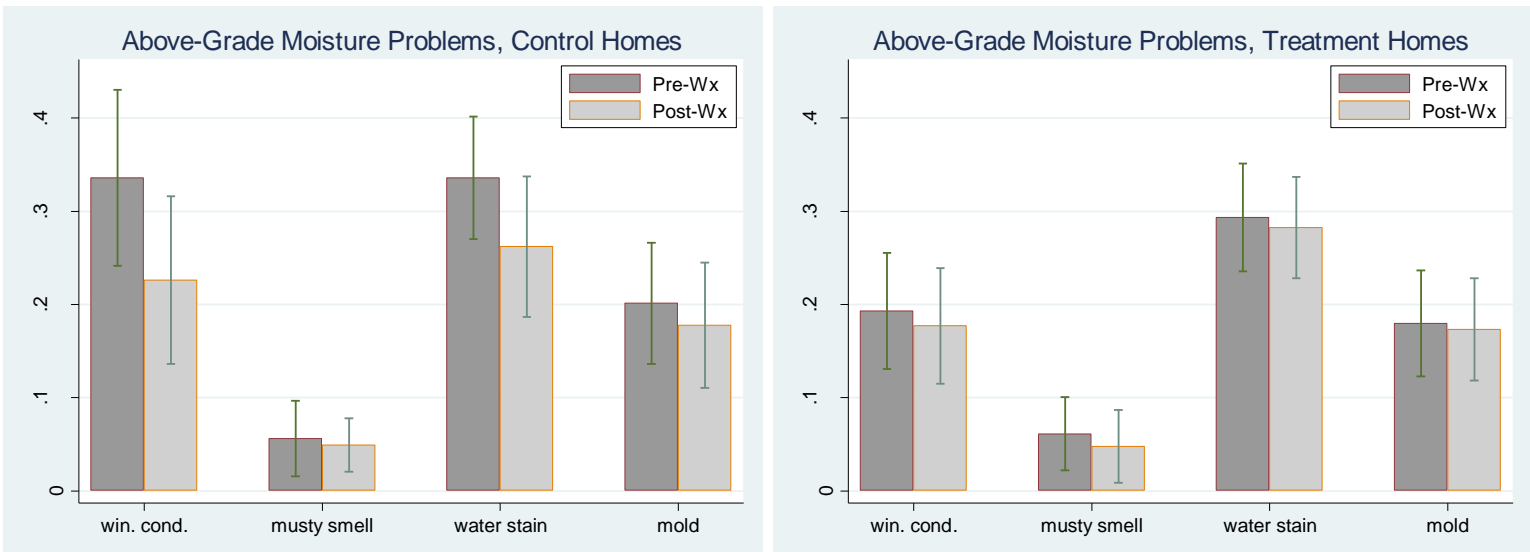

Fig. 30. Incidence of recorded above-grade moisture problems for sites with the same technician pre- and post-weatherization (with $90 \%$ confidence intervals).
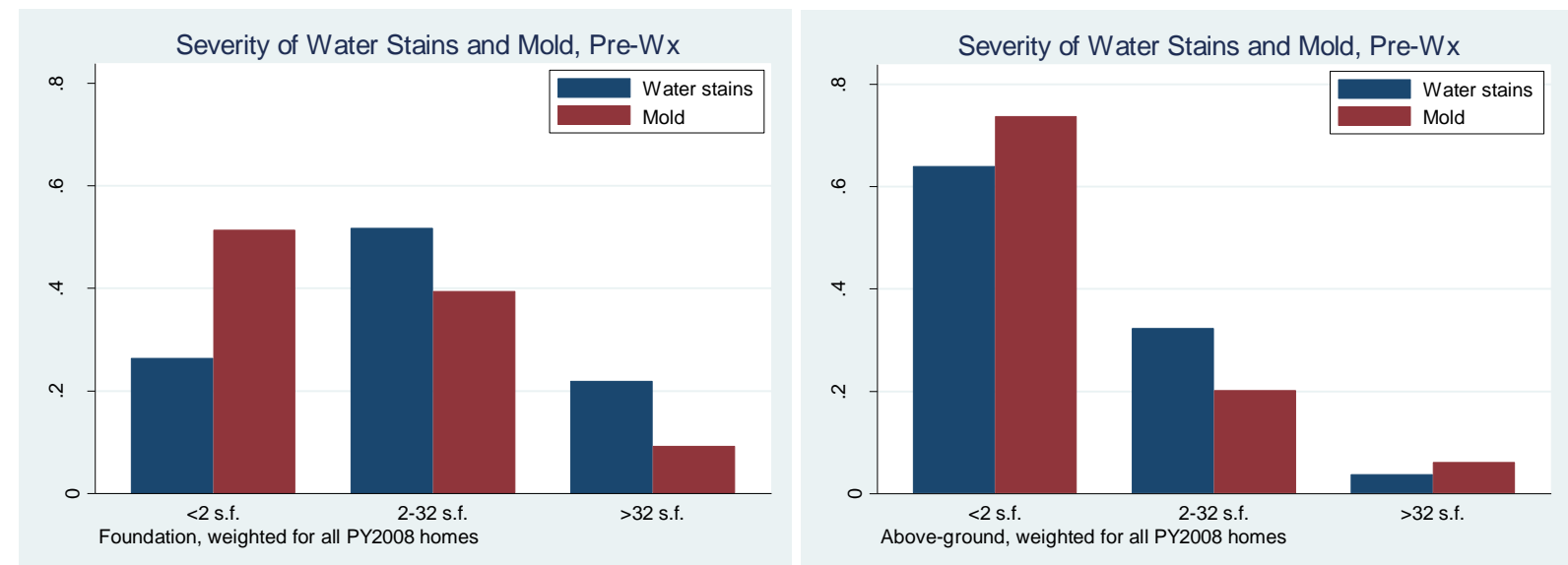

Fig. 31. Pre-weatherization recorded severity of water stains and mold for foundation spaces (left) and above grade (right). 



\section{DISCUSSION}

Following is a qualitative discussion of some of the key findings and limitations of the study.

\section{Carbon Monoxide}

The testing revealed a small proportion of homes with indoor combustion devices that produce carbon monoxide at significant levels, and an even smaller proportion of homes with measurable $\mathrm{CO}$ in the ambient indoor air. In some cases, elevated indoor CO could be traced to a likely source, such as $\mathrm{CO}$ produced by a heating system, migration from an attached garage or use of a gas oven: in other cases, there was no obvious explanation. However, given the potential deadly nature of carbon monoxide, the incidence of CO-related problems found in the study homes does suggest maintaining the combustionsafety testing procedures under the program.

It is very challenging for both statistical and ethical reasons to properly document the impact that weatherization has on indoor CO. Statistically rare events, such as severe CO problems, are hard to study - for example, if CO poisoning occurs in 1 in every 1000 homes, then one cannot expect to measure the change in frequency of such events based on studying 500 homes. More importantly though, the significant safety concern related to $\mathrm{CO}$ meant that the study could not passively observe what happened when, for example, a study technician measured high $\mathrm{CO}$ in a heating system. The hazard notifications that the study itself generated had an impact on the outcomes of the homes in the study, including immediate action in some homes in the control group.

\section{Radon}

The data from the study indicate that weatherization increases radon levels in homes on average, and that the magnitude of the impact is generally proportional to the reduction in natural ventilation that results from air-sealing work. This is not to say that all air-sealing work necessarily serves to increase radon concentrations in homes: below-grade sealing and sealing between living spaces and unoccupied foundation spaces may well help reduce radon levels in living spaces. Also, the study was implemented prior to implementation of the ASHRAE 62.2 standard for whole-house ventilation (ASHRAE 2010b) that DOE is currently phasing into the program. Further research is needed to understand the impact of implementation of this standard on indoor radon levels (and other indoor contaminants), but the results from the 21 homes in the study known to have received such ventilation is at least suggestive of a beneficial impact of this measure.

No correlation was found between changes in radon levels and the installation of ground covers over dirt floors and below-grade sealing, but this finding should not be taken as an indictment of the potential for these measures to help reduce radon levels. Since radon was likely not the primary purpose for installing these measures in the study homes, they may have been applied in ways that were ineffective in the context of radon. For example, to be an effective radon barrier, ground covers need to be tightly sealed to foundation walls - a detail that is less critical in the primary application of ground covers for mitigating moisture migration. Though most experts agree that an active soil depressurization system is the preferred approach to remediating indoor radon, more research is needed to gauge the potential for lower-cost measures such as ground covers and below-grade sealing to help offset weatherization-related increases in radon.

\section{Formaldehyde}

This study measured formaldehyde levels in weatherization homes that are comparable to those found in other studies of existing homes - and far less than the levels that raised alarms about temporary housing 
units provided by FEMA following hurricane Katrina, which averaged more than four times the levels found here. However, the lack of a generally accepted standard for indoor residential formaldehyde does make interpretation of these data difficult. Also, the relatively small sample of sites tested for formaldehyde in this study limits the conclusions that can be drawn. The data from (the even more limited) sample of mobile homes in the study suggests that formaldehyde levels in mobile homes may be higher, and that weatherization may have a larger impact in this housing stock. But additional testing on a larger sample of mobile homes would be needed to reach a firm conclusion on this.

\section{Temperature}

While the indoor temperature data from the study reveal a wide range of indoor temperatures across weatherization homes, temperature generally changed little immediately following weatherization. The small $\left(0.3^{\circ} \mathrm{F}\right)$ but statistically significant observed change in indoor temperature in the data could be the result of some households choosing a higher thermostat setpoint following weatherization, but could also easily simply reflect improved thermal integrity from weatherization. For example, temperatures decay more slowly during thermostat setback periods in better-insulated homes. Either way, the observed change is very small. Note, however, that the study gathered data for an average of only about four weeks preceding and following weatherization, and thus the results here do not reflect any longer-term effects that may be associated with weatherization.

\section{Humidity and Moisture}

The study provides evidence of a small increase in indoor humidity during the heating season following weatherization, which is a benefit, because the majority of program homes in heating climates were very dry prior to weatherization. The observed humidity impact is somewhat smaller than a simple moisture balance calculation would suggest, and bears additional investigation.

The moisture-observation data gathered during the fieldwork is useful in roughly characterizing the general incidence of moisture-related issues in program homes, but the subjective nature of the observations - and the relative rarity in the sample of active moisture issues around the time of weatherization - makes it difficult to draw substantive conclusions about the impact of weatherization on indoor moisture issues. 


\section{REFERENCES}

American Society of Heating, Refrigerating and Air Conditioning Engineers (ASHRAE) 2009. 2009 ASHRAE Handbook - Fundamentals (I-P Edition), American Society of Heating, Refrigerating and AirConditioning Engineers, Inc.

American Society of Heating, Refrigerating and Air Conditioning Engineers (ASHRAE) 2010a. Thermal Environmental Conditions for Human Occupancy, ANSI/ASHRAE Standard 55-2010. Available from www.ashrae.org.

American Society of Heating, Refrigerating and Air Conditioning Engineers (ASHRAE) 2010b. Ventilation and Acceptable Indoor Air Quality in Low-Rise Residential Buildings, ANSI/ASHRAE Standard 62.2-2010. Available from www.ashrae.org.

Arvela, H. (1995). Seasonal Variation in Radon Concentration of 3000 Dwellings with Model Comparisons. Radiation Protection Dosimetry, 33-42.

Building Performance Institute (BPI) 2012. "Combustion Safety Test Procedure for Vented Appliances," http://www.bpi.org/files/pdf/CombustionSafetyTestProcedure-GoldSheet.pdf, accessed March 20, 2012.

California Environmental Protection Agency, Office of Environmental Health Hazard Assessment (OEHHA) 1999. Acute Reference Exposure Level (RELs). Air Toxics Hot Spots Program Risk Assessment Guidelines. Part II. Technical Support Document for Describing Available Cancer Potency Factors. OEHHA, Sacramento, CA.

Chi, P. S. (1990). Energy Efficiency and Radon Risks in Residential Housing. Energy Vol. 15, No. 2, 81 89.

Chia-yu Lin, A. G. (1999). Analysis of Local Decisions Using Hierarchical Modeling, Applied to Home Radon Measurement and Remediation. Statistical Science, Vol. 14, No. 3 , pp. 305-328.

Environmental Protection Agency (EPA) 2011a. http://www.epa.gov/radon/healthrisks.html, accessed July 7, 2011.

(U.S.) Environmental Protection Agency (EPA) 2009. “A Citizen's Guide To Radon: The Guide To Protecting Yourself And Your Family From Radon,” Publication EPA 402/K-09/001, January 2009.

Health Canada. 2006. Residential Indoor Air Quality Guideline: Formaldehyde. Ottawa: Health Canada.

Hun, D.E., R.L. Corsi, M.T. Morandi, and J.A. Siegel. 2010. "Formaldehyde in residences: long-term indoor concentrations and influencing factors," Indoor Air 2010; 20: 196-203.

Karg, Rick 2001. "Proposed Field Protocol for Gas Range Carbon Monoxide Emissions Testing," Report prepared for the Chicago Regional Diagnostics Working Group, July 2001.

http://www.karg.com/pdf/CO Field Protocol.pdf, accessed March 20, 2012.

Krewski, D., Mallick, R. A., Zielinski, J. M., \& Letourneau, E. G. (2005). Modeling Seasonal Variation in Indoor Radon Concentrations. Journal of Exposure Analysis and Environmental Epidemilogy, 2324-243.

Nero, A. E. (1990). Indoor Radon and Decay Products: Concentrations, Causes, and Control Strategies. Washington, D.C.: Department of Energy. 
OEHHA California Office of Environmental Health Hazard Assessment. "All OEHHA Acute, 8-hour and Chronic Reference Exposure Levels (chRELs) as on December 18, 2008.” (Accessed Dec 7, 2011)

Offerman, Francis J. 2009. Ventilation and Indoor Air Quality in Homes. California Energy Commission and California Air Resource Board. November. CEC 500-2009-085.

(OSHA) U.S. Department of Labor, Occupational Safety \&Health Administration. "Regulations (Standards - 29 CFR).” (Accessed Dec 7, 2011).

http://www.osha.gov/pls/oshaweb/owadisp.show document?p id=10075\&p table=STANDARDS

Ternes, Mark P, Martin Schweitzer, Bruce Tonn, Richard Schmoyer, and Joel F. Eisenberg. 2007. "National Evaluation of the Weatherization Assistance Program: Preliminary Evaluation Plan for Program Year 2006," ORNL/CON-498. Available at: http://weatherization.ornl.gov/pdfs/ORNL CON498.pdf.

World Health Organization (WHO). 2009. WHO Handbook on Indoor Radon: A Public Health Perspective. World Health Organization, Geneva Switzerland. 


\section{APPENDIX A. SAMPLING, WEIGHTING AND UNCERTAINTY ESTIMATION PROCEDURES}





\section{APPENDIX A. SAMPLING, WEIGHTING AND UNCERTAINTY ESTIMATION PROCEDURES}

This appendix describes the sampling and sample weighting procedures used in the study, along with the methods used to calculate sampling-error based confidence intervals.

\section{SAMPLING}

Sampling for the study was implemented in two stages: first, a primary sample of geographic areas was sampled; second individual households were sampled and recruited for the study from within these sampled geographic areas.

The primary sampling unit (PSU) for the study was a Census Bureau super-PUMA. Super-PUMAs are contiguous, within-state geographic areas defined by the US Census Bureau containing a minimum of 400,000 persons and designed for use with Public Use Microdata Sample (PUMS) data disseminated by the Bureau. Super-PUMAs are aggregations of smaller Public Use Microdata Areas (PUMAs).

We selected Super-PUMAs as the geographic PSU for the study for two reasons: (1) when considering trade-offs between geographic diversity versus cost and logistical considerations, super-PUMAs represent a reasonable geographic size for the study (smaller than states but larger than counties); and, (2) the PUMS data associated with each super-PUMA can be used to estimate the WAP-eligible population in the region, which is useful for the sampling procedure we will describe shortly.

We drew a stratified, probability-proportional-to-size (PPS) sample of Census 2000 super-PUMAs for the study, as described in the following sections.

\section{Measure of Size}

The geographic sampling for the study was probability-proportional-to-size; that is, the probability of selecting a given super-PUMA is proportional to the "size" of the PSU (super-PUMA), where size is here defined as the total weatherization funding for weatherizing single-family homes in PY08.

To develop a measure of size for all lower-48 super-PUMAs, we first used household-level data from the 2006-2008 American Community Survey (ACS) to estimate the total population of weatherizationeligible households in each super-PUMA (both single- and multi-family).

Next, we estimated the total PY08 weatherization funding in each PSU as follows:

- We allocated published state-level PY08 funding allocations to local weatherization agencies proportionally to agency funding levels reported to us by states in the All States Agencies Information Survey implemented as part of the overall PY08 program evaluation effort. For a few states where these data were not available, we used prior planned agency allocations reported to DOE.

- We allocated these PY08 local agency funding estimates to counties (proportionally to Census 2000 population) based on the service territory for each local agency. Where more than one agency served a county, we assumed an equal division of county population among agencies.

- We rolled the county-level estimates of PY08 weatherization funding up to the PSU level using the Census 2000 PUMA equivalency files to map counties to super-PUMAs. Where a county contained more than one PSU, we allocated funding proportionally according to Census 2000 population.

We then calculated our measure of "size" for each PSU as: 
- PY08 funding * (single-family, weatherization-eligible households)/(all weatherization-eligible households)

\section{Stratification by Radon zone}

In order to obtain the desired over-sample of homes in high radon areas, it was necessary to classify each PSU by radon potential. EPA classifies each US county into one of three zones, based on predicted average radon screening levels ${ }^{32}$ :

- $\quad$ EPA Zone $1 \longrightarrow 4 \mathrm{pCi} / 1$

- $\quad$ EPA Zone $2-2-4 \mathrm{pCi} / 1$

- $\quad$ EPA Zone $3-<2 \mathrm{pCi} / 1$

Similarly, Lawrence Berkeley National Laboratory (LBNL) has modeled average radon levels at the county-level. ${ }^{33}$ We used the LBNL estimates of the percentage of homes in each county with an expected long-term radon concentration at or above $4 \mathrm{pCi} / 1$ to define three LBNL categories:

- $\quad$ LBNL-based Category $1 \longrightarrow>20 \%$ of homes $\geq 4 \mathrm{pCi} / 1$

- $\quad$ LBNL-based Category $2-5-20 \%$

- LBNL-based Category $3-<5 \%$

To roll the county-level data up to the PSU level, we calculated the county-population weighted average EPA and LBL zone for each super-PUMA, rounding to the nearest zone designation. We then divided all lower-48 US super-PUMAs into four radon strata as shown in Table A.1. Radon strata for sampling

Table A.1. Radon strata for sampling.

\begin{tabular}{llccc}
\hline & \multicolumn{3}{c}{ LBNL-based Category } \\
\cline { 2 - 5 } & & $\mathbf{1}$ & $\mathbf{2}$ & $\mathbf{3}$ \\
\hline \multirow{2}{*}{ EPA } & 1 & very high & high & high \\
Zone & 2 & high & mid & mid \\
& 3 & high & mid & low \\
\hline
\end{tabular}

Figure A.1 shows how the super-PUMAs are classified under this stratification scheme.

\footnotetext{
${ }^{32}$ http://www.epa.gov/radon/zonemap.html
}

33 http://eetd.lbl.gov/IEP/high-radon/hr.html 


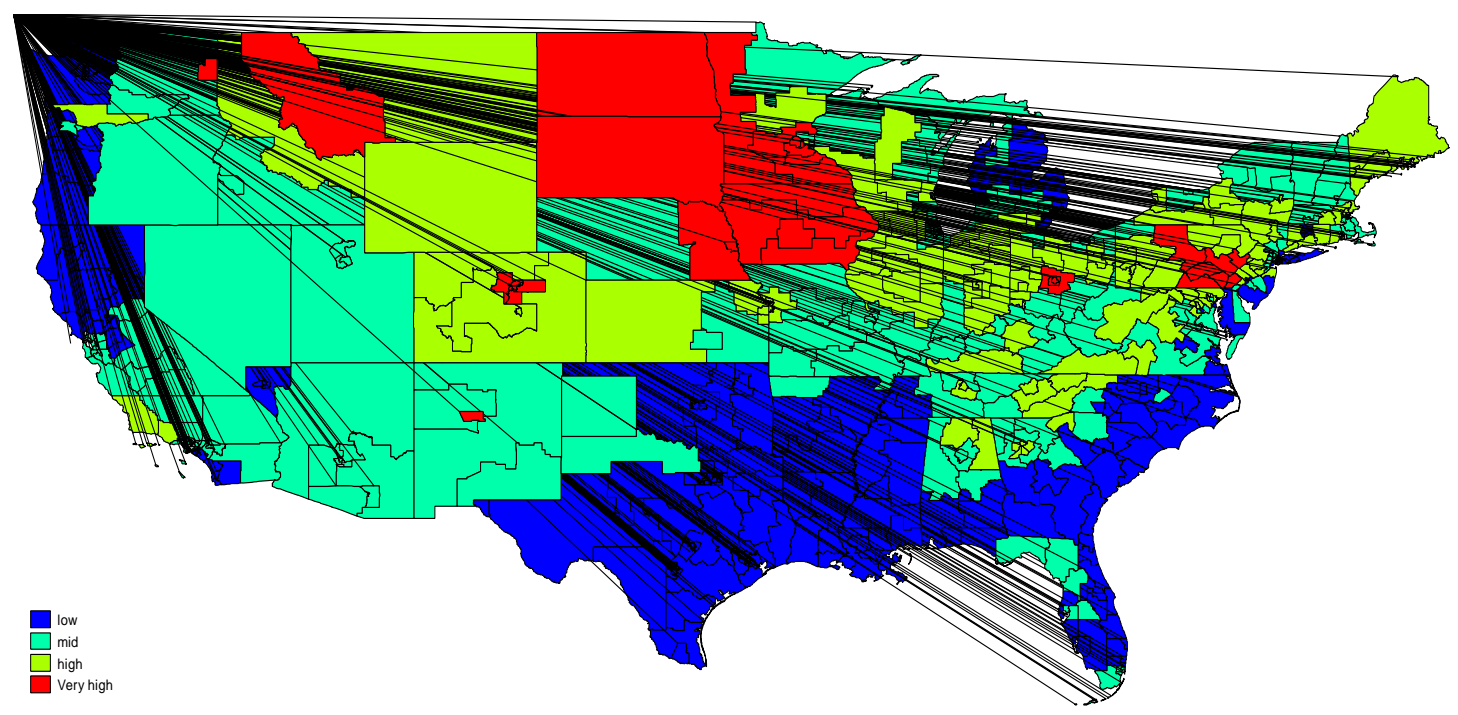

Fig. A.1. Super-PUMA classification by radon stratum.

We divided the target sample of 80 PSUs (later reduced for budget reasons to 77) into two halves. The first half served as a nationally representative sample, and was allocated to each stratum in proportion to the stratum total measure of size. The second half was used to formulate an oversample of high-radon areas. For this, we omitted the lowest radon stratum, and allocated PSUs to the remaining strata in proportion to the estimated number of homes in each stratum with radon levels exceeding $4 \mathrm{pCi} / \mathrm{l}$ (using the LBNL county data and aggregating counties to super-PUMAs as described above). Table A.2. PSU (super-PUMA) allocations to radon strata shows these stratum allocations.

Table A.2. PSU (super-PUMA) allocations to radon strata.

\begin{tabular}{rccc}
\hline Radon Stratum & Representative Sample & Radon Oversample & Total \\
\hline Low & 9 & 0 & 9 \\
Mid & 14 & 9 & 23 \\
High & 12 & 16 & 28 \\
Very high & 5 & 15 & 20 \\
Total & 40 & 40 & 80 \\
\hline
\end{tabular}

\section{PPS Sampling}

PSUs (super-PUMAs) were sampled systematically within radon stratum. For each of the four radon strata, we:

1. calculated the sampling interval as the total stratum measure of size (MOS) divided by the number of PSUs to be sampled;

2. sorted the list of PSUs in the following order:
a. Census division
b. climate region ${ }^{34}$
c. state $^{35}$

\footnotetext{
${ }^{34}$ Climate zones were defined as follows: Zone 1- ID, ME, MI, MN, MT, ND, NH, NY, SD, VT, WI, WY - Zone 2: CO, CT, IA, IL, IN, MA, NE, NJ, NV, OH, PA, RI, UT; Zone 3 - AR, DC, DE, KS, KY, MD, MO, NC, OK, TN, VA, WV; Zone 4 AL, FL, GA, LA, MS, SC, TX; Zone 5: AZ, CA, NM, OR, WA.
} 

d. average January temperature ${ }^{36}$

3. created a running sum of MOS across the sorted list of PSUs, such that each PSU was indexed to the running sum and represented by a portion of the index equal to that PSUs MOS;

4. selected a random starting value within the first sampling interval; and,

5. sampled PSUs starting with the PSU represented by the random starting point of the index and then continuing through the list at equal sampling intervals calculated in Step 1 above.

Note that this approach allows for a PSU to be sampled more than once if its MOS is larger than the sampling interval. Two such PSUs were sampled twice because of this: one in Minnesota and one in South Dakota. These two areas were allocated twice the number of sites as other PSUs.

We later made one ad hoc substitution: a sampled super-PUMA in Delaware was determined to have no local weatherization agencies that could assist with the project: we therefore randomly selected one of the PSUs on either side of the originally sampled one.

Also, for budgetary reasons, three sampled super-PUMAs in the south that were selected for coolingseason data collection were later dropped from the study.

\section{WEIGHTING}

The analysis of the study data incorporates case weights to appropriately represent the population of single-family homes treated by the program in PY08. In particular, for inferences about the program population as a whole, it is important to account for the fact that homes in high radon areas are more heavily represented in the study sample than they are in the overall population of homes treated by the program. A case weight represents the number of homes in the population represented by each home in the study sample.

In general, case weights for probability samples like this one are given by the inverse of the probability of selection. For the sampling approach used here, the probability of selecting a particular home in the study is the product of the probability of selecting the PSU (super-PUMA) in which the home resides and the probability of selecting the home among all homes treated by the program in that PSU.

The probability of selecting a given sampled super-PUMA can be readily calculated from the PPS sampling procedure described above. However, the probability of selecting a home within a super-PUMA is not readily known, because sites for the study were recruited from lists of near-term candidate sites rather than complete lists of all homes to be treated by the program.

We therefore estimated a pseudo-probability of selection at the individual house level, derived from the ratio of the number of study sites in each super-PUMA to the estimated total population of PY08 singlefamily completions in the super-PUMA. Here, it is noteworthy that - while the original super-PUMA sampling had to rely on estimates of weatherization-eligible households for sampling purposes - by the time fieldwork for the study was completed, better estimates of actual weatherization completions at the agency level were available. These came from the All Agencies Program Information Survey and the compilation of lists of completed housing units obtained from a subset of sampled agencies. We mapped these local-agency-level data to the super-PUMA level using the procedures described above.

\footnotetext{
${ }^{35}$ For this procedure, the following states were aggregated into a single super-state: AZ, CA, NM, OR and WA.

${ }^{36}$ Based on aggregating county-level average temperatures (from http://eetd.lbl.gov/iep/high-radon/files.html ) to the superPUMA level using county population proportions.
} 
We created two sets of probability case weights for analyzing data from the study. The first pooled the treatment and control groups, and was used for inferring pre-weatherization characteristics of program homes (where the random treatment/control assignment is immaterial). The second treated the two groups as separate samples, each to be weighted to the population level. These weights were used for inferring changes associated with weatherization. Statistics on the range of these weights are provided in Table A.3.

Table A.3. Probability case weights.

\begin{tabular}{rccc}
\hline & Pooled & Treatment Group & Control Group \\
\hline Cases & 505 & 322 & 183 \\
Mean weight & 151.1 & 237.0 & 417.0 \\
Median weight & 118.6 & 191.0 & 343.3 \\
Minimum weight & 26.1 & 41.8 & 69.7 \\
Maximum weight & 530.6 & 849.0 & $1,415.0$ \\
\hline
\end{tabular}

In addition, we post-stratified and weighted the sample in two dimensions: EPA radon zone and mobile home versus site-built housing to ensure that the sample weighted up to the best-available estimate of total PY08 single-family housing unit completions. For EPA radon zone population estimates, we used the agency-level completion data on single-family homes and agency-to-county mapping procedures described above to estimate the population of program homes by EPA radon zone. We also used these data along with DOE state-level tracking system data as the population estimate for single-family, sitebuilt and mobile home completions in PY08. The population estimates used for post-stratification and weighting are shown in Table A.4. PY08 Population estimates used for post-weighting.

Table A.4. PY08 Population estimates used for post-weighting.

\begin{tabular}{rrrr}
\hline & Site-built & Mobile home & \multicolumn{1}{c}{ Total } \\
\hline EPA Zone 1 & 25,705 & 6,621 & 32,326 \\
EPA Zone 2 & 23,619 & 7,315 & 30,934 \\
EPA Zone 3 & 13,204 & 3,673 & 16,877 \\
Total & 62,528 & 17,609 & 80,137 \\
\hline
\end{tabular}

\section{STATISTICAL PRECISION CALCULATIONS}

Statistical confidence intervals reported in this report are at a 90 percent confidence interval, and incorporate sampling error at the primary (super-PUMA) and secondary (site) sampling levels.

Specifically, confidence intervals are based on the SVY command in the Stata (Version 12) statistics software package, set as follows:

- $\quad$ stratification - super-PUMA

- probability weight - either pooled or separate treatment/control probability weights described above;

- variance estimation - Taylor linearized;

- handling of strata with single PSU - scaled;

- finite-population correction - applied at super-PUMA level

- $\quad$ post-stratification and weighting - post-strata and post-stratification population weights as described above 
$\bullet$ 
APPENDIX B. ANALYSIS OF SITES WITH ELEVATED AMBIENT CARBON MONOXIDE 



\section{APPENDIX B. ANALYSIS OF SITES WITH ELEVATED AMBIENT CARBON MONOXIDE}

This appendix provides additional detail regarding sites in the study with logged elevated CO, either persistently or episodically.

Carbon monoxide issues were not noted in many homes. We identified four types of CO results as worthy of further investigation. Only 34 homes were noted to have one or more of these conditions. These four types are:

1. Average over the sample period of more than $3 \mathrm{ppm}$ ( 4 homes pre-Wx, 7 post-Wx)

2. Five or more events over the sample period with a reading over $20 \mathrm{ppm}$ ( 11 homes)

3. Any event of $35 \mathrm{ppm}$ or more (18 pre-Wx, 7 post-Wx)

4. An change in average $\mathrm{CO}$ level from pre-Wx to post-Wx of more than $1 \mathrm{ppm}$ (16 homes)

These are shown in Table B.1. 
Table B.1 Carbon monoxide issues not noted in many homes.

\begin{tabular}{|c|c|c|c|c|c|c|}
\hline \multirow[t]{2}{*}{ Site } & \multicolumn{2}{|c|}{$\begin{array}{c}\text { At least one event over } 35 \\
\text { ppm }\end{array}$} & \multirow[t]{2}{*}{$\begin{array}{l}5 \text { or more events } \\
\text { over } 20 \mathrm{ppm}\end{array}$} & \multicolumn{2}{|c|}{ Average over 3 ppm } & \multirow{2}{*}{$\begin{array}{r}\text { Change of } 1 \\
\text { ppm or more }\end{array}$} \\
\hline & Pre-Wx & Post-Wx & & Pre-Wx & Post-Wx & \\
\hline $2323-I 22$ & & $\mathrm{X}$ & & & & \\
\hline 2348-I11 & $\mathrm{X}$ & & & & & \\
\hline 2348-I23 & & & & & $\mathrm{X}$ & $\mathrm{X}$ \\
\hline 2713-I05 & & & & & $\mathrm{X}$ & $\mathrm{X}$ \\
\hline 2713-I17 & $\mathrm{X}$ & & & & & \\
\hline 2899-I01 & $X$ & & & & & \\
\hline 2899-I15 & $X$ & & $\mathrm{X}$ & & & $\mathrm{X}$ \\
\hline 2967-I02 & $\mathrm{X}$ & & $\mathrm{X}$ & & & \\
\hline 3370-I07 & & & & & & $\mathrm{X}$ \\
\hline 3824-I03 & & & & & $\mathrm{X}$ & $X$ \\
\hline 3832-I02 & & & & & $X$ & $\mathrm{X}$ \\
\hline 3863-I01 & $\mathrm{X}$ & & & & & \\
\hline 5313-I20 & & & & $\mathrm{X}$ & $\mathrm{X}$ & $\mathrm{X}$ \\
\hline 5333-I05 & $\mathrm{X}$ & $\mathrm{X}$ & $\mathrm{X}$ & & & \\
\hline $5423-\mathrm{I} 11$ & $\mathrm{X}$ & $\mathrm{X}$ & $\mathrm{X}$ & & & \\
\hline 5431-I05 & $X$ & & $\mathrm{X}$ & $\mathrm{X}$ & & $\mathrm{X}$ \\
\hline 5654-I01 & $\mathrm{X}$ & & $\mathrm{X}$ & & & \\
\hline 5654-I06 & & & & & & $\mathrm{X}$ \\
\hline $5680-\mathrm{I} 06$ & $X$ & & & & & \\
\hline $5680-\mathrm{I} 12$ & $\mathrm{X}$ & & $\mathrm{X}$ & & & \\
\hline 6946-I04 & & $\mathrm{X}$ & & & & \\
\hline 7654-I04 & & & & & $\mathrm{X}$ & $\mathrm{X}$ \\
\hline $7654-\mathrm{I} 12$ & & & & & & $\mathrm{X}$ \\
\hline 7692-I05 & & & & & & $\mathrm{X}$ \\
\hline 7996-I15 & & & & & & $\mathrm{X}$ \\
\hline $8184-\mathrm{I} 05$ & & $\mathrm{X}$ & & & & \\
\hline 8325-I08 & $\mathrm{X}$ & & $\mathrm{X}$ & $\mathrm{X}$ & & $\mathrm{X}$ \\
\hline $8387-\mathrm{I} 06$ & & & & & & $\mathrm{X}$ \\
\hline $8388-\mathrm{I} 15$ & & & $\mathrm{X}$ & & & \\
\hline 8398-I01 & $\mathrm{X}$ & & & & & \\
\hline 9262-I07 & $X$ & $\mathrm{X}$ & $\mathrm{X}$ & $\mathrm{X}$ & $\mathrm{X}$ & $\mathrm{X}$ \\
\hline 9289-I21 & $\mathrm{X}$ & & & & & \\
\hline 9626-I20 & $X$ & & & & & \\
\hline 9795-I03 & $X$ & $X$ & $\mathrm{X}$ & & & \\
\hline
\end{tabular}




\section{Average values over 3 ppm for sampling period:}

Table B. 2 shows the sites that had over 3 ppm CO in the pre-Wx period, the post-Wx period, or both.

Table B.2 Sites over 3 ppm CO in pre-Wx period, post-Wx period, or both.

\begin{tabular}{cccc}
\hline Site ID & Control/Treatment & Pre-Wx CO (avg. ppm) & Post-Wx CO (avg. ppm) \\
\hline 2348 -I23 & Treatment & 0.0 & 3.7 \\
$2713-$ I05 & Treatment & 0.4 & 5.4 \\
$3824-I 03$ & Control & 0.8 & 6.4 \\
$3832-$ I02 & Control & 2.2 & 6.2 \\
$5313-I 20$ & Control & 10.7 & 11.8 \\
$5431-I 05$ & Treatment & 18.8 & 0.0 \\
$7654-I 04$ & Treatment & 0.0 & 4.2 \\
$8325-$ I08 & Treatment & 7.7 & 0.3 \\
9262-I07 & Treatment & 5.5 & 12.5 \\
\hline
\end{tabular}

Of these 9 homes, 6 had substantial increases between pre- and post-Wx, one had a modest increase (but was very high both pre- and post-Wx, and two had large drops.

The two that had large drops (5431-I05 and 8325-I08) may be related to furnace replacement performed at these sites.

Three of the homes (3824-I03, 5313-I20, and 7654-I04) all had unvented space heaters. As of the time of this study unvented space heaters were allowed to remain in homes if they were used for supplemental heat as opposed to being the primary heat source. Changes in pre-post ambient CO levels may have been driven by changes in space heater usage in any of these homes. Two of the three homes were control homes, so air tightening is a likely factor in only one of the three cases. CO In the case of 3824-I03, the unvented heater was a kerosene unit and the homeowner did not always have fuel for the heater, and changes in fuel availability may be a factor.

Site 2348-I23 is an all-electric mobile home and the occupants indicate that people are not allowed to smoke inside. Site 2713-I05also lacks a clear explanation. This home had a natural gas furnace and water heater but an electric range and clothes dryer. The furnace was induced draft, combustion safety tests did not indicate a problem in this home, and the $\mathrm{CO}$ in the water heater flue was only $4 \mathrm{ppm}$ - certainly not enough to explain an ambient average of $5.4 \mathrm{ppm}$. We suspect that poor performance of monitoring devices may explain these observations.

Site 3832-I02 had noticeable levels both pre- and post-Wx, though pre-Wx the average was below 3 ppm. This home had a propane range. Since this home was also a control home the changes are not related to weatherization.

Site 9262-I07 had an oven that showed high CO emissions, and these emissions increased greatly in the post-Wx period. At the pre-Wx visit the oven was measured to have $1442 \mathrm{ppm}$ (air-free) CO and this increased to $3740 \mathrm{ppm}$ (air-free) after weatherization. 


\section{Five or more events over 20 ppm:}

There were 11 homes with 5 or more events of 20 ppm or more. These are shown in Table B.3.

Table B.3 Homes with 5 or more events of $20 \mathrm{ppm}$ of $\mathrm{CO}$ or more.

\begin{tabular}{lcc}
\hline Site ID & Control/Treatment & \# events \\
\hline $2899-$ I15 & Control & 7 \\
$2967-I 02$ & Treatment & 45 \\
$5333-$ I05 & Control & 15 \\
$5423-I 11$ & Control & 12 \\
$5431-I 05$ & Treatment & 352 \\
$5654-I 01$ & Control & 6 \\
$5680-I 12$ & Control & 13 \\
$8325-I 08$ & Treatment & 10 \\
$8388-I 15$ & Treatment & 5 \\
$9262-I 07$ & Treatment & 54 \\
$9795-I 03$ & Treatment & 40 \\
\hline
\end{tabular}

Of the eleven homes with five or more events over $20 \mathrm{ppm}$, three have already been discussed: 5431-I05 (which had 352 events over 20 ppm) and 8325-I08 (10 events) had the furnace replaced, and 9262-I07 (54 events) with the increase in oven emissions. The other 8 homes did not have average CO levels above 3 ppm despite having several events above $20 \mathrm{ppm}$.

Site 2899-I15 had 7 events. This home had an unvented heater. This was a control home, so the heater remained in place throughout the study.

Site 2967-I02 had 45 events. At this home the post-Wx test of the oven measured 2883 ppm (air-free) CO. There was no oven test pre-Wx.

Site 5333-I05 had 15 events. At this home there is no indication of the cause. The range was electric and there was no garage. There was also no evidence of combustion safety problems with the furnace or water heater, and the occupants stated that smoking was not permitted in the home. None of the events lasted for more than several minutes.

Site 5423-I11 had 12 events. No indication of cause could be identified, though some of these events were for extended periods of time. There was no attached garage, $\mathrm{CO}$ readings for the range top burners and oven were low, the water heater was electric, and the furnace was induced draft. No answers about smoking habits were provided.

Site 5654-I01 had 6 events. This was a control home. The combustion safety testing did not suggest a problem with the furnace, and the only other combustion appliance in the home was a range with low emissions. There was no garage. It is not clear what caused the CO spikes at this home.

Site 5680-I12 had 13 events. No indication of cause could be identified, though some of these events were for extended periods of time. There was no attached garage, $\mathrm{CO}$ readings for the range top burners and oven were low, the water heater was electric, and the furnace was induced draft. The occupants said that no smoking was allowed in the home.

Site 8388-I15 had 5 events, all of which were barely over $20 \mathrm{ppm}$. This home had a natural gas furnace and water heater. The furnace was replaced during weatherization. There were no other combustion 
appliances in the home. There was an attached garage. It is most likely that the spikes were from the garage since the combustion safety testing showed strong drafts, but this is not certain.

Site 9795 -I03 had 40 events. This home was using a wood stove due to the oil furnace having a cracked heat exchanger. There were no other combustion appliances in the home and no attached garage. The wood furnace was the most likely cause of the events, probably during tending of the wood.

\section{Any event over 35 ppm:}

There were 21 homes with 1 or more events of $35 \mathrm{ppm}$ or more. These are shown in Table B.4. A level of $35 \mathrm{ppm}$ was chosen for this evaluation since that is the ambient level at which it is common for assessors to stop combustion testing.

Table B.4 Homes with 1 or more events of 35 ppm CO or more.

\begin{tabular}{|c|c|c|c|}
\hline Site ID & Control/Treatment & Max Pre-Wx CO (ppm) & Max Post-Wx CO (ppm) \\
\hline $2323-I 22$ & Control & 5.0 & 78.0 \\
\hline 2348-I23 & Treatment & 37.5 & 9.0 \\
\hline 2713-I17 & Treatment & 35.0 & 25.0 \\
\hline 2899-I01 & Treatment & 36.5 & 0.0 \\
\hline 2899-I05 & Control & 63.0 & 13.0 \\
\hline 2967-I02 & Treatment & 79.0 & No reading \\
\hline 3863-I01 & Treatment & 42.0 & 0.0 \\
\hline 5333-I05 & Control & 67.5 & 59.5 \\
\hline 5423-I11 & Control & 44.5 & 36.0 \\
\hline 5431-I05 & Treatment & 18.8 & 0.0 \\
\hline 5654-I01 & Control & 54.0 & 0.0 \\
\hline $5680-\mathrm{I} 06$ & Control & 57.0 & 0.0 \\
\hline $5680-\mathrm{I} 12$ & Control & 45.0 & 5.0 \\
\hline 6946-I04 & Treatment & 0.0 & 48.0 \\
\hline 8184-I05 & Treatment & 10.5 & 44.5 \\
\hline $8325-\mathrm{I} 08$ & Treatment & 7.7 & 0.3 \\
\hline 8398-I01 & Treatment & 65.0 & 11.0 \\
\hline 9262-I07 & Treatment & 67.0 & 67.5 \\
\hline 9289-I21 & Treatment & 49.5 & 30.5 \\
\hline 9626-I20 & Treatment & 89.5 & 0.0 \\
\hline 9795-I03 & Treatment & 39.0 & 52.0 \\
\hline
\end{tabular}

Of these 21 homes, ten have been discussed above and the other 11 did not have as many as 5 spikes throughout the test period.

Of these 11, seven had attached garages (2323-I22, 2348-I11, 2713-I17, 5680-I06, 8398-I01, 9289-I21, 9626-I20). Of those seven, only three homes (2323-I22, 2713-I17, 9289-I21) had any spikes over 20 ppm in the post-Wx period. One of these, 2323-I22, only had an elevated event in the post-Wx period, and this was a control home. These results suggest that there was enough leakage between the garages and homes to have elevated $\mathrm{CO}$ on an occasional basis, most likely when the car was started in the garage. Only two of the homes that have been discussed previously (5431-I05 and 5680-I12) also had attached garages.

In one home, 9626-I20, the home had no door between the home and the garage in the pre-Wx period and there were several spikes with much lower CO levels. This was corrected by weatherization. 
Of the remaining 4 homes, 2899-I01 received a new furnace and there was no evidence of anything else that could contribute to the high spike.

Site 3863-I01 was an all-electric home and there was no evidence of why there would be a high CO spike.

Site 6946-I04 had an oven with exceptionally high emissions (25470 ppm (air-free)) post-Wx, but was otherwise an all-electric home. The project team arranged for repairs and testing of this oven after the field technician reported these results. High $\mathrm{CO}$ production was due to a improper burner orifice.

Site 8184-I05 is an all-electric mobile home, and the occupants said that no smoking was allowed in the home. The cause of high $\mathrm{CO}$ spikes in unclear.

\section{Homes with a change of at least 1 ppm:}

There were 16 homes with changes of $1 \mathrm{ppm}$ or more between the pre-Wx and post-Wx periods. These are shown in Table B.5.

Table B.5 Homes with changes of $1 \mathrm{ppm}$ or more between the pre-Wx and post-Wx periods.

\begin{tabular}{|c|c|c|c|}
\hline Site ID & Control/Treatment & Pre-Wx CO (avg. ppm) & Post-Wx CO (avg. ppm) \\
\hline $2348-I 23$ & Treatment & 0.0 & 3.7 \\
\hline 2713-I05 & Treatment & 0.4 & 5.4 \\
\hline 2899-I15 & Control & 1.5 & 0.1 \\
\hline 3370-I07 & Control & 0.5 & 1.7 \\
\hline 3824-I03 & Control & 0.8 & 6.4 \\
\hline 3832-I02 & Control & 2.2 & 6.2 \\
\hline $5313-\mathrm{I} 20$ & Control & 10.7 & 11.8 \\
\hline $5431-\mathrm{I} 05$ & Treatment & 18.8 & 0.0 \\
\hline 5654-I06 & Treatment & 2.0 & 0.0 \\
\hline 7654-I04 & Treatment & 0.0 & 4.2 \\
\hline 7654-I12 & Treatment & 0.0 & 1.9 \\
\hline 7692-I05 & Treatment & 1.9 & 0.3 \\
\hline 7996-I15 & Treatment & 0.2 & 1.3 \\
\hline $8325-\mathrm{I} 08$ & Treatment & 7.7 & 0.3 \\
\hline 8387-I06 & Treatment & 0.4 & 2.4 \\
\hline 9262-I07 & Treatment & 5.5 & 12.5 \\
\hline
\end{tabular}

All but 6 of these homes (3370-I07, 5654-I06, 7654-I12, 7692-I05, 7996-I15, 8387-I06) have been discussed previously. For the remaining 6 homes the average readings were low and there were few elevated events, so even these changes of $1 \mathrm{ppm}$ or more were of low levels of $\mathrm{CO}$.

Two of these homes, 5654-I06 and 7692-I05, received new furnaces as a part of weatherization.

Sites 3370-I07 and 7654-I12 each had two events that exceeded $20 \mathrm{ppm}$ and had natural gas ranges. The events of more than $20 \mathrm{ppm}$ occurred in the general vicinity of dinnertime. Changes in oven may explain the difference in CO levels. Site 3370-I07 was a control home so the changes were not due to weatherization. Site 7654-I12 was a treatment home.

Site 7996-I15 had an oil furnace. It is not clear what may have caused the slightly elevated post-Wx average $\mathrm{CO}$ level. 
Site 8387-I06 did not have a specific finding pointing to the elevated CO level post-Wx, but the home had many natural gas appliances so usage patterns are likely. The air leakage of the home changed only $19 \%$ between pre-Wx and post-Wx.

\section{Summary:}

Taken together, these findings suggest that attached garages may be the cause of individual events but are rarely related to long-term low levels of $\mathrm{CO}$ in these homes. Gas ranges and furnaces were the most likely causes of persistent elevated $\mathrm{CO}$ levels. Usage patterns of ranges can have a significant impact on $\mathrm{CO}$ levels in homes. 




\section{APPENDIX C. RADON LITERATURE REVIEW}

\section{Radon}

Radon $_{222 \text { (half life } 3.8 \text { days) }}$ is a naturally occurring, colorless, odorless, inert, radioactive gas. It is part of the radioactive decay chain that originates with uranium 238 (half life- 4.5 billion years). Radon is formed by the radioactive decay of radium 226 (half life -1602 years). Radium $_{226}$ is frequently found as a trace element bound in soils and rock. Although radium may be found in some building materials radon entering a building from below grade is the radon source for the vast majority of problem buildings. When radium 226 undergoes radioactive decay it releases radon, which as an inert gas can migrate through the air in the pores of soils and fissures in bedrock. Radon decays into a series of short lived decay products - polonium 218 (half life 3.1

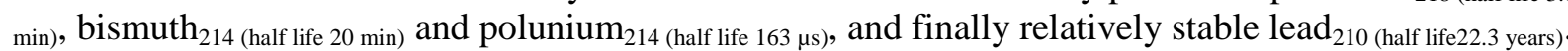
The combination of radon's 3.8 day half life and the very short half lives of the bismuth and polonium isotopes allows radon to enter buildings from surrounding soil and rock where the short lived decay products are likely to decay while still inside the building. Depending on the radon concentration in the pore space of surrounding soil and rock and how much of the infiltrating air passing through a building enters from below grade, radon levels inside building may be high enough to deliver a significant radiation dose to the lungs of occupants. This is the heart of the concern about indoor radon.

Radon laden soil gas entering buildings through below grade cracks and holes in structural foundations is the most frequent source of elevated indoor radon. In a small fraction of cases radon outgassing from well water or emitted from building materials is the primary source in buildings with elevated radon levels. Radon dissolved in water readily outgasses to the atmosphere, so the only water sources at risk are those with deep wells in bedrock in which the water is not exposed to the atmosphere until it emerges in the shower, sink, toilet, dishwasher or clothes washer. Most public water supplies are exposed to air in reservoirs or aquifers. Aggregate used to manufacture concrete and concrete products may contain enough radium $_{228}$ to result in elevated indoor radon concentrations. The original cases of houses with elevated radon levels were in locations where uranium mine tailing were used to make concrete and concrete products (Angell, 2008).

\section{Health Impacts of Radon}

The Environmental Protection Agency (EPA) and World Health Organization (WHO) both recognize radon, and its decay products, as the second leading cause of lung cancer after cigarette smoking (EPA 2011, WHO 2009). The number of homes in the U.S. that exceeds the $4 \mathrm{pCi} / \mathrm{L}$ threshold is one in fifteen $(\text { PEPPS })^{37}$. When radon levels were measured by tenure status on homes in nonmetropolitan areas of New York City (Table C.1) it was found that a higher proportion of rental units and lower priced owneroccupied homes were in the high-risk categories for radon (Chi \& Laquatra, 1990).

\footnotetext{
${ }^{37}$ Program For Environmental Policy And Planning Systems (PEPPS) http://www.pepps.fsu.edu/segip/states/NC/radon.html
} 
Table C.1 Radon levels by tenure status and housing value - 245 households in nonmetropolitan counties of New York (Chi and Laquatra 1990).

\begin{tabular}{|l|l|l|}
\hline $\begin{array}{l}\text { Tenure and Value of } \\
\text { Households }\end{array}$ & Low $(<4 \mathrm{pCi} / \mathrm{L})$ & High $(>4 \mathrm{pCi} / \mathrm{L})$ \\
\hline Rental Units & 33.86 & 66.14 \\
\hline $\begin{array}{l}\text { Owner occupied units valued } \\
\text { less than } \$ 40,000\end{array}$ & 58.77 & 41.23 \\
\hline $\begin{array}{l}\text { Owner occupied units valued } \\
\text { less than } \$ 40,000\end{array}$ & 63.77 & 36.23 \\
\hline
\end{tabular}

Exposure to radon and the short lived decay products has been linked to increased lung cancer risk in uranium miners since the 1920s (Akerblom 1999). Since that time many studies have been conducted to examine health effects resulting from radon exposure. Several literature reviews by cognizant bodies have been performed during that time period (Committee on the Biological Effects of Ionizing Radiation (BEIR) IV 1988, BEIR VI 1999, WHO 2009). Based on the BEIR IV literature review, the International Agency for Research on Cancer classified radon as a human carcinogen (IARC 1988).

In 1999 the EPA asked the National Academies of Science to conduct a review of the medical literature to determine what the potential health risk to the population exposed to radon concentrations at the generally lower concentrations found in dwellings. The National Research Council convened the Biological Effects of Ionizing Radiation VI committee (BEIR VI 1999). To develop a dose risks model the BIER VI committee:

- reviewed the genomic mechanism that induce cancer in lung tissue

- reviewed animal studies

- reviewed residential case-control studies

- pooled the data from 11 miner studies and developed a methodology for meta-analysis; collectively the studies included 68,000 men, 2,700 of whom died from lung cancer.

The committee findings include:

- $\quad$ sufficient laboratory evidence to identify biological mechanisms for radon and decay product related damage genomic damage that could lead to development of lung cancer

- residential case control studies reviewed were not sufficient to derive a valid risk estimate

- miner studies supplemented by laboratory studies on the development of radon related lung cancer were sufficient to derive valid risk estimates. The committee central risk estimates were 15,400 to 21,800 radon related lung cancer deaths per year. Uncertainty analysis results in estimates that could be as low as 3,000 lung cancer deaths per year and as high as 33,000 lung cancer deaths per year.

- Many of the radon related lung cancer deaths occur in tobacco smokers 
In 2009 the World Health Organization published the Who Handbook on Indoor Radon (WHO 2009). The Handbook was developed by the WHO Department of Public Health and Environment within the framework of the WHO International Radon Project. The WHO Handbook reviews the BEIR VI report plus studies completed since the BEIR VI report was completed. Additional studies included analysis of residential case-control studies and follow up work with German miners (see Table C.2). The WHO analysis depends heavily on pooled analysis of residential case-control studies in the US (Krewski 2005, 2006), China (Lubin 2004) and Europe (Darby 2005, 2006). WHO concludes that:

- Epidemiological studies confirm that radon in homes increases the risk of lung cancer in the general population.

- Other health effects of radon have not consistently been demonstrated.

- The proportion of all lung cancers linked to radon is estimated to lie between $3 \%$ and $14 \%$, depending on the average radon concentration in the country and on the method of calculation.

- Radon is the second most important cause of lung cancer after smoking in many countries.

- Radon is much more likely to cause lung cancer in people who smoke, or who have smoked in the past, than in lifelong non-smokers. However, it is the primary cause of lung cancer among people who have never smoked.

- There is no known threshold concentration below which radon exposure presents no risk. Even low concentrations of radon can result in a small increase in the risk of lung cancer.

- The majority of radon-induced lung cancers are caused by low and moderate radon concentrations rather than by high radon concentrations, because in general less people are exposed to high indoor radon concentrations. 
Table C.2 WHO Handbook compares estimated excess lung cancer rates reported from existing studies (Zeeb 2009).

\begin{tabular}{|c|c|c|c|c|c|c|}
\hline & \multirow[t]{2}{*}{$\begin{array}{l}\text { Nbr. of } \\
\text { studies } \\
\text { included }\end{array}$} & \multirow[t]{2}{*}{$\begin{array}{l}\text { Nbr. of } \\
\text { lung } \\
\text { cancers }\end{array}$} & \multirow[t]{2}{*}{$\begin{array}{l}\text { Nbr. of } \\
\text { controls }\end{array}$} & \multirow[t]{2}{*}{$\begin{array}{l}\text { Exposure } \\
\text { Window } \\
\text { (years) }^{\mathrm{a}}\end{array}$} & \multicolumn{2}{|c|}{$\begin{array}{l}\text { Percentage increase in risk of } \\
\text { lung cancer per } 100 \mathrm{~Bq} / \mathrm{m}^{3} \\
\text { increase in radon concentration }\end{array}$} \\
\hline & & & & & $\begin{array}{l}\text { Based on } \\
\text { measured radon }\end{array}$ & $\begin{array}{l}\text { Based on long- } \\
\text { term average } \\
\text { radon }^{\mathrm{b}}\end{array}$ \\
\hline \multicolumn{7}{|c|}{ Pooled analyses of studies of indoor radon in the home } \\
\hline $\begin{array}{l}\text { European } \\
\text { (Darby et al. } \\
2005,2006)\end{array}$ & 13 & 7148 & 14208 & $5-35$ & $8(3,16)$ & $16(5,31)$ \\
\hline $\begin{array}{l}\text { North American } \\
\text { (Krewski et al. } \\
2005,2006 \text { ) }\end{array}$ & 7 & 3662 & 4966 & $5-30$ & $11(0,28)$ & - \\
\hline $\begin{array}{l}\text { Chinese } \\
\text { (Lubin et al. 2004) }\end{array}$ & 2 & 1050 & 1995 & $5-30$ & $13(1,36)$ & - \\
\hline $\begin{array}{l}\text { Weighted average } \\
\text { of above results } \\
\text { of pooling studies }\end{array}$ & & & & & 10 & $\sim 20^{\circ}$ \\
\hline \multicolumn{7}{|c|}{ Studies of radon exposed miners ${ }^{\mathrm{d}, \mathrm{e}}$} \\
\hline $\begin{array}{l}\text { BEIR VI analysis } \\
\text { (BEIR VI 1999; } \\
\text { Lubin et al. 1997) }\end{array}$ & 11 & 2787 & & $5-35$ & \multicolumn{2}{|c|}{$\begin{array}{l}\text { All miners: } 5 \\
\text { Miners exposed to }<50 \text { WLM only: } \\
14 \text { Miners exposed to }<50 \mathrm{WLM} \text { and } \\
\text { at }<0.5 \mathrm{WL} \text { only: } 30\end{array}$} \\
\hline $\begin{array}{l}\text { German uranium } \\
\text { miners study } \\
\text { (Grosche et al. 2006) }\end{array}$ & 1 & 2388 & & $5-35$ & \multicolumn{2}{|c|}{$\begin{array}{l}\text { All miners: } 3 \\
\text { Miners with low exposures incurred } \\
\text { at low dose rates: } 18^{r}\end{array}$} \\
\hline $\begin{array}{l}\text { French and Czech } \\
\text { uranium miners } \\
\text { (Tomasek et al. 2008) }\end{array}$ & 2 & 574 & & $5+5-35$ & \multicolumn{2}{|c|}{$\begin{array}{l}\text { All miners (mean exposure } \\
\text { rate } 4.5 \mathrm{WLM} / \text { year): } 32\end{array}$} \\
\hline
\end{tabular}

a i.e. considering radon concentrations during the period starting 35 years before and ending 5 years before the date of diagnosis for cases of lung cancer, or a comparable date for controls.

b i.e. adjusting for year-to-year random variability in indoor radon concentration

${ }^{c}$ Informal estimate, indicating the likely effect of removing the bias induced by random year-to-year variation in radon concentration.

${ }^{d}$ Risks per WLM have been converted to risks per $100 \mathrm{~Bq} / \mathrm{m}^{3}$ by assuming that $1 \mathrm{~Bq} / \mathrm{m}^{3}$ at equilibrium is equivalent to $0.00027 \mathrm{WL}$, that the "equilibrium factor" in dwellings is 0.40 , that subjects spend $70 \%$ of the time at home, that there are $365.25 \times 24 / 170=51.6$ 'Working Months' in one year, and that the ratio of the dose to lung cells for exposures in homes to that for similar exposures in mines (sometimes referred to as the Kfactor) is unity.

- Only one study has specifically addressed the effect of measurement error in the estimates of radon-related lung cancer risk in miners (Stram et al. 1999). This concluded that for miners exposed at concentrations below 15 WL measurement error was of little consequence.

' Informal estimate, obtained by multiplying the estimate for all miners in the German cohort by 6, i.e. the ratio of the estimates for all miners and for miners exposed to $<50$ WLM and $<0.5 \mathrm{WL}$ from the BEIR VI analysis. 


\section{Radon Policy}

Primary EPA radon policy is contained in two documents: A Citizen's Guide to Radon and The Home Buyer's and Seller's Guide to Radon. The basis for the policies presented in these two documents is found in The EPA Radon Reference Manual (EPA 1987).

The Citizen's guide contains the following key messages:

- $\quad$ Radon is a naturally occurring radioactive gas

- It is sometimes found in houses at levels that present an significant increased risk of dying from lung cancer

- The EPA recommends testing all houses for radon

- The EPA recommends mitigating any building with an annual average radon concentration greater than $4 \mathrm{pCi} / \mathrm{L}$

- Long term radon tests ( $>90$ days) better reflect the annual average concentration better than short term tests ( 2 to 90 days)

- Recommends three step testing:

- Conduct short term screening test. If the short term screening test is less than $4 \mathrm{pCi} / \mathrm{L}$, the house is considered to be below the EPA action level and you're done;

○ Decide whether to conduct follow up testing:

- if the test is greater than or equal to $4 \mathrm{pCi} / \mathrm{L}$ conduct a follow up test to confirm the finding;

- a long term test better represents the annual average

- a short term follow up test adds more certainty to a short term screening test

- the higher the result of the screening test the more likely the annual average is to exceed $4 \mathrm{pCi} / \mathrm{L}$. If the screening test is $>8 \mathrm{pCi} / \mathrm{L}$, conduct a short term follow up test.

- Decide whether to mitigate:

- If a long term follow up test result is greater than or equal to $4 \mathrm{pCi} / \mathrm{L}$ fix it

- If the average of a short term follow up test and the screening test is greater than or equal to $4 \mathrm{pCi} / \mathrm{L}$ consider fixing the house.

- Radon in homes can be fixed using soil depressurization techniques.

- Preferred test setup - test on the lowest lived-in level, closed house conditions, heating and cooling systems on, ventilation fans off, do not test during severe storms or windy periods 
The Home Buyer's and Seller's Guide to Radon contains the following recommendations:

- Test a house for radon at the time of sale

- Fix it if the testing results are greater than or equal to $4 \mathrm{pCi} / \mathrm{L}$

- Ask for radon resistant new house features if buying a newly constructed home

- Take steps to prevent tampering with tests

The suggested threshold for radon remediation proposed by EPA is $4 \mathrm{pCi} / \mathrm{L}$ was established during the agency's first uranium mine tailings remediation project in the 1970's. It was found that the remediation efforts for uranium-contaminated buildings would reduce average indoor radon concentrations below this level (Angell, 2008). The threshold that is still used as a reference level today is based upon technology used forty years ago. Recommended threshold levels for radon remediation vary by country.The World Health Organization recommends that governments create radon programs and establish a national reference level of $100 \mathrm{~Bq} / \mathrm{m}^{3}(2.7 \mathrm{pCi} / \mathrm{L})$. Wherever this is not possible, the chosen level should not exceed $300 \mathrm{~Bq} / \mathrm{m}^{3}$ (8.1 pCi/L) (WHO 2009). Many European countries already have recommended reference levels or enforceable reference levels. Figure C.1 lists those in effect in 2005 (Synnott 2005). Canada recommending action at $20 \mathrm{pCi} / \mathrm{L}$ (Chia-yu Lin, 1999).

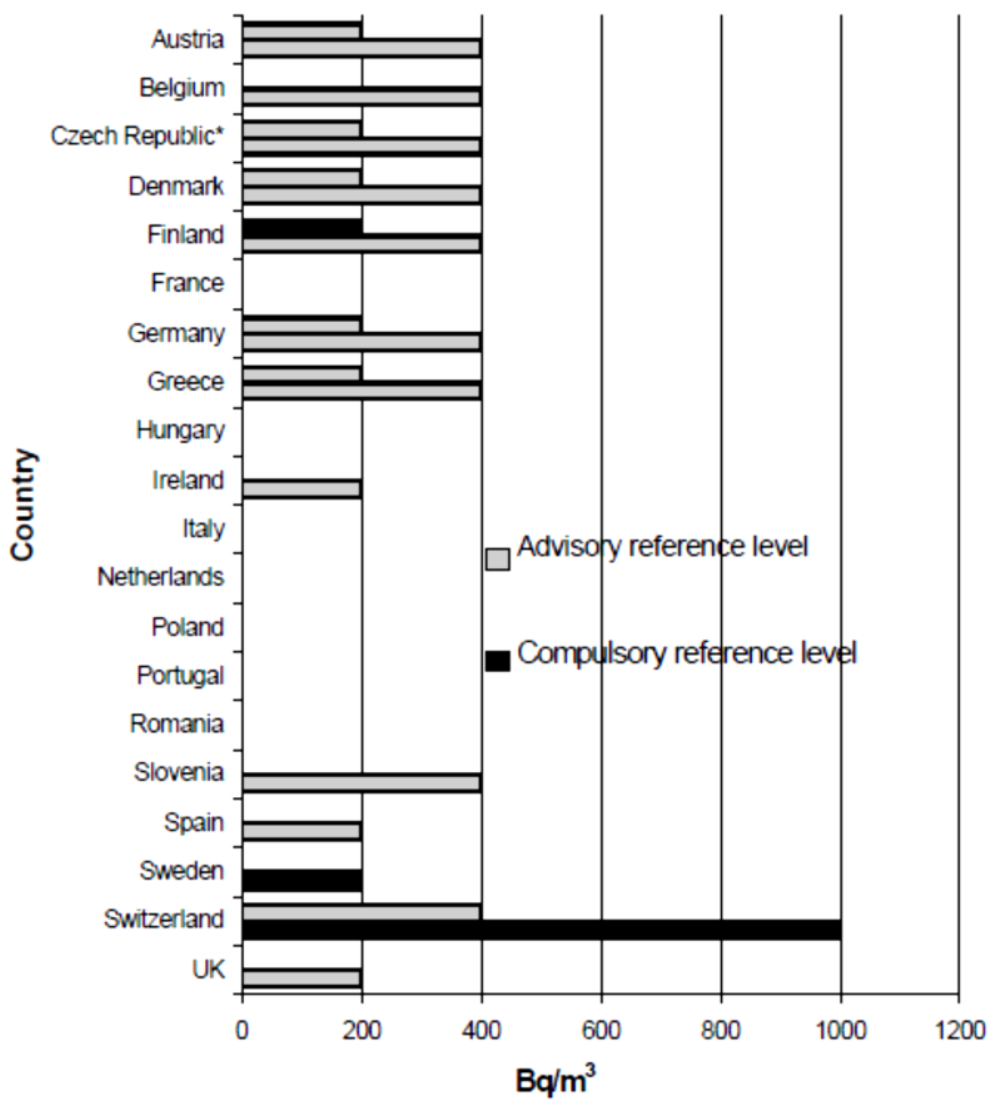

Fig. C.1 Recommended radon reference levels by country.

A number of US states have enacted statutes that cover a range of radon related activities. The most common statutes cover testing and disclosure at the time of real estate transactions and qualifications for those measuring or remediating radon. 
The primary defining moment of US radon policy came with the enactment of the Indoor Radon Remediation Act (IRRA) of 1988 (Angell, 2008). This Act authorized EPA to provide 14 million annually to states to provide radon programs, testing/surveys, radon mitigation, technical assistance, research and public education. Under IRRA, Congress delegated all regulatory action to be left to the states. Since then less than half of the states have enacted regulations to license radon remediation/testing professionals. As of 2008, in the U.S., there were 6000 radon testing professionals and 2000 radon mitigation providers.

\section{Indoor Radon Dynamics}

Geographical distribution: Radon contamination shares several characteristics with other environmental hazards:

- the risks are geographically dispersed but have strong spatial patterns

- techniques for identification of risky areas already exist, but it is difficult to identify high risk households

- localized measurements can be done to identify the risks of individual households

In order for radon to be an indoor problem there must be a source of radium nearby or in the house itself. Studies have been conducted that combine indoor radon surveys and surfical uranium data to produce radon risk maps (EPA 1993). The maps identify areas that are more prone to indoor radon problems than other areas. However indoor radon levels can vary significantly from one home to another within the same neighborhood. Chi and Laquatra (1990) pronounced that an accurate prediction of radon levels to a particular area is without any scientific foundation and was said to be "impossible". The only way to determine the concentration of radon or its decay products in a specific building is to measure for radon or its decay products in the particular building.

\section{Radon in buildings:}

Radon concentration in individual buildings varies by location within the building and over time. For the vast majority of buildings the source of radon is radon laden air entering from the soil. It is not surprising that many studies have found that radon levels in spaces with the greatest contact with the earth have the highest radon concentrations - e.g. basements and the first floor of slab on grade buildings (Ronca-Batista 1990). The ratio of radon concentration in basements to first floor is typically a factor of two, but ranges from 1 - 4. For this reason it is important to define where a radon sample being used to compare to the EPA guideline of $4 \mathrm{pCi} / \mathrm{L}$ annual average should be taken so it reflects radon levels to which occupants are likely to be exposed. The EPA Citizen's guide recommends placing detectors in the lowest lived in level.

Radon concentration over time varies directly with the rate at which it enters a building and inversely with rate at which the contaminant is removed by ventilation. The source term for indoor radon largely depends on the radon concentration and amount of infiltrating air that passes through the surrounding soil or air permeable bedrock before entering a building. Infiltrating air enters a building from above grade and below grade air leaks. Outdoor air enters a building when the indoor air is lower pressure than the outdoor air. The pressure differences drive air infiltration are most frequently induced by stack effect, wind, the operation of ventilation fans and the operation of air distribution systems.

The fraction of the infiltrating air that enters from below grade depends on the air flow characteristics of the above and below grade leaks and the indoor - outdoor pressure differences experienced by the above 
and below grade air leaks. Typically a few percent of the total infiltration air enters from below grade. A research project in houses in the Spokane River Valley found that in buildings situated on highly permeable material (e.g. glacial till, river run gravel, shattered shale or limestone) larger fractions - as high as $25 \%$ - of the infiltrating air comes from below grade (Turk 1988). The indoor-outdoor pressure difference is affected by outdoor air temperature, wind, and the operation of mechanical systems and appliances that exhaust air from houses and whether windows are open or closed (Turk 1987, Turk 1988).

Radon concentrations in soil air vary from a few hundred pCi/L to tens of thousands of pCi/L (Turk 1988, Dudley 1989). For most US houses radon levels will be between 1 and $3 \mathrm{pCi} / 1$. Soil gas radon concentrations 100 to $200 \mathrm{pCi} / \mathrm{L}$ in combination with below grade infiltration of less than $1 \%$ of total air infiltration would result in radon concentrations in this range. For indoor radon to be elevated above the 4 $\mathrm{pCi} / \mathrm{L}$ guideline the soil air concentration, the below grade infiltration rate or both must be higher. For example, a $5 \%$ below grade infiltration rate at 100 to $200 \mathrm{pCi} / \mathrm{L}$ soil air concentration would result in indoor levels between 5 and $10 \mathrm{pCi} / \mathrm{L}$; while a half percent below grade infiltration rate in combination with $10,000 \mathrm{pCi} / \mathrm{L}$ results in something closer to $50 \mathrm{pCi} / \mathrm{L}$.

As the magnitude of negative pressure in a building increases, air is drawn in from outside. Air drawn in from above grade dilutes the indoor radon concentration. Air drawn in from below grade may increase, decrease or have no net effect on the rate at which radon enters the building. Whether indoor radon levels increase or not depends on whether increased soil air entry compensates for above grade dilution. Whether the radon source term increases or not depends on whether the increased flow through the soil collects radon from radium deposits that were just a bit too far from the building to be participating at smaller pressure differences. So if no new radon sources are added to the air flowing through the soil, then the soil air concentration will be reduced by dilution with outdoor air and the source term goes down. If enough new radon sources are added to balance the dilution rate then the radon source term remains the same, but at higher flow rates and lower concentration. If significant new radon sources are included in the increased soil air flow then radon source term increases. It may increase enough to overwhelm the increased leakage from above grade leaks. The source term and the ventilation rate are not independent variables.

Ventilation rates in single family residences are dominated by open windows and by pressure differences accidentally induced by stack effect, wind pressures and the operation of air distribution systems. As indoor-outdoor pressure differences vary the amount of radon entering the building varies and the amount of dilution air entering the building varies. It is easy to see that radon levels in houses will vary over time and from floor to floor within the building.

Radon fluctuates over the course of hours, days, weeks and seasons (Scott 1993, EPA 1986). Figure C.2 presents an illustration of radon variation over time. It shows hourly radon measurements made by Arthur Scott in a basement in New York State from November 25, 1992 to February 13, 1993 (Scott 1993). Radon concentrations vary from a low of around $1 \mathrm{pCi} / \mathrm{L}$ to a high of $15 \mathrm{pCi} / \mathrm{L}$, with a mean of $4-$ $6 \mathrm{pCi} / \mathrm{L}$. It is clear radon concentrations vary on a daily basis. Typical daily variations are on the order of $3-5 \mathrm{pCi} / \mathrm{L}$, but in some instances they range as high as $10 \mathrm{pCi} / \mathrm{L}$. Notice that there are also periods of relative stability lasting several days - e.g. December $15^{\text {th }}$ through December $27^{\text {th }}$ and January $15^{\text {th }}$ through the $20^{\text {th }}$ are fairly stable at around $4 \mathrm{pCi} / \mathrm{L}$ and December $7^{\text {th }}$ through the $14^{\text {th }}$ and January $3^{\text {rd }}$ through January $10^{\text {th }}$ are more variable periods with higher concentrations. These cycles are most likely driven by weather systems passing through the area. Last, notice that the mean radon concentration trends down over the entire period. This is a seasonal trend. 


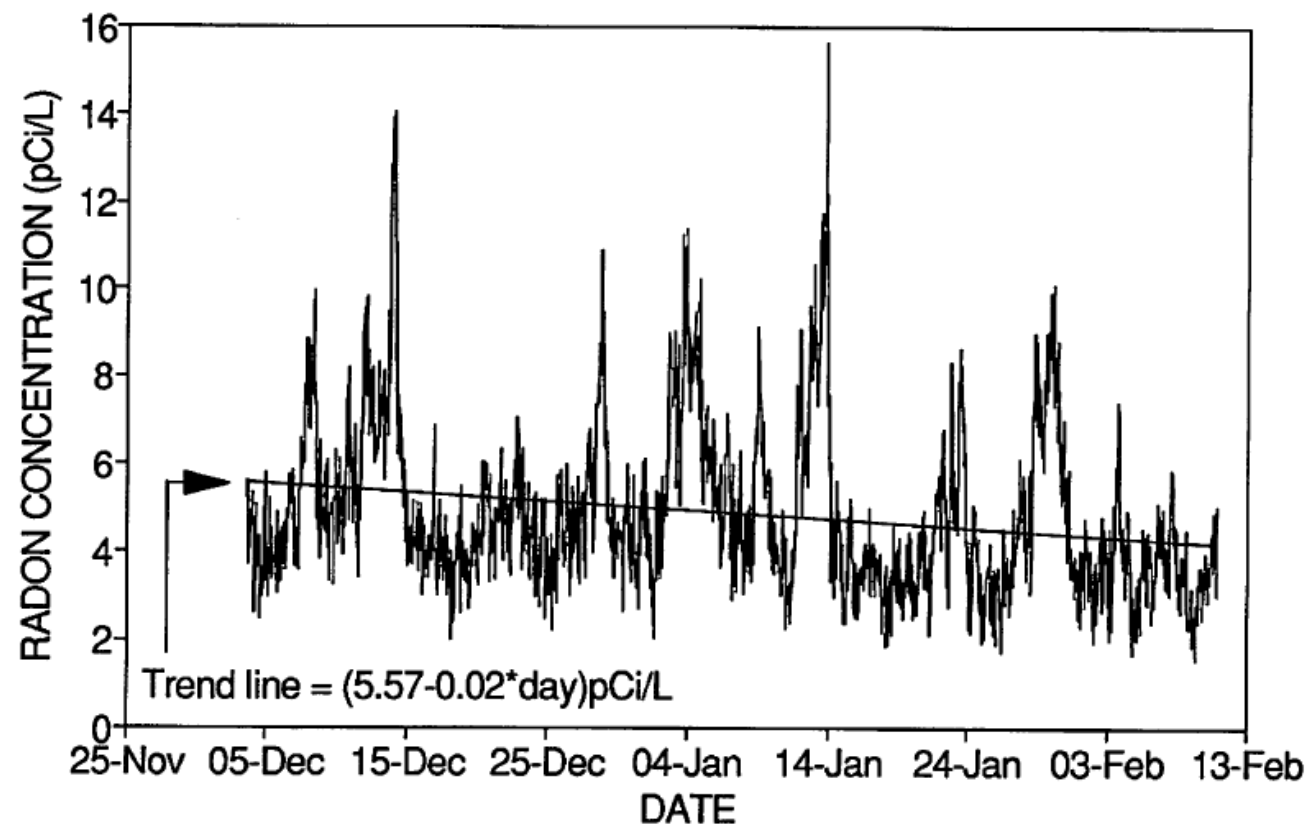

Fig. C.2 Hourly radon measurements made by Arthur Scott in a basement in New York State from November 25, 1992 to February 13, 1993 (Scott 1993).

Indoor radon concentrations can sometimes be radically affected by occupant behavior or the operation of mechanical systems. A typical residence has an effective total leakage area of something less than 2 square feet. Opening one window doubles the leakage area. Opening five increases the leakage area by a factor of ten. Opening windows during mild weather is a seasonal effect that has been seen in numerous radon studies (EPA 1986, Arvela, 1995, Belanger 1990, Bierma 1989, Borak 1989, Hull 1989, Krewski 2005, Lin 1998, Roessler 1987, Ronca-Batista 1988, 1990). This produces a wintertime peak concentration and a summertime low concentration. Soil air radon concentrations may also peak in the winter and contribute to this effect. In other studies this trend is less pronounced. Roessler (1987) reports finding summer peaking, winter peaking and non-peaking radon concentrations in a set of 37 houses with 12 monthly charcoal canister tests (Fig. C.3). Steck (1990) reports normal distribution of monthly charcoal canister measurements for most houses in an 84 house study with 15\% showing a strong difference between summer and winter concentrations. At least one of these houses exhibited a summer radon peak. 


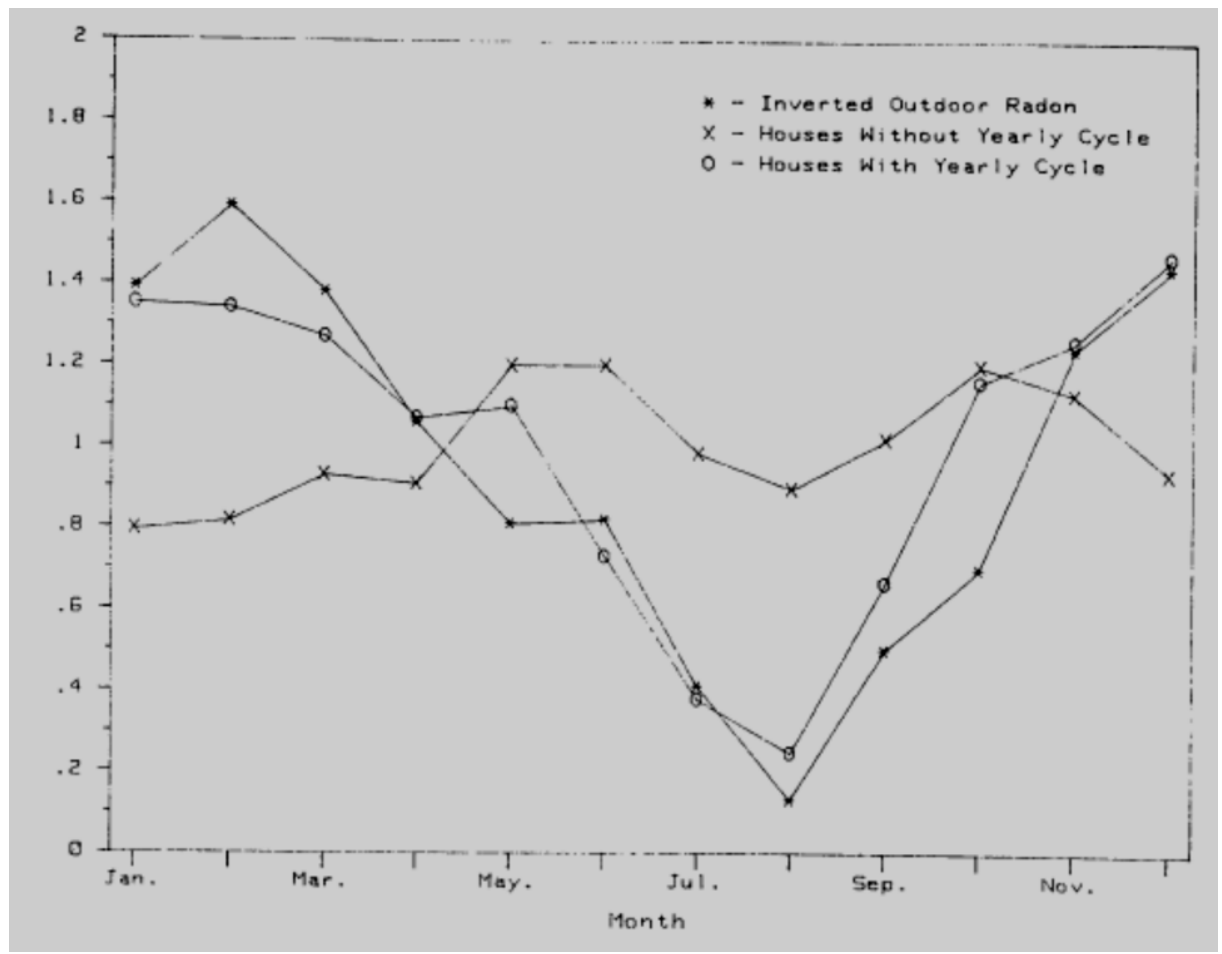

Fig. C.3 Normalized radon concentrations for the 20 houses in the EPA seasonal variation study in Montana (EPA 1986). 17 of the houses showed winter peaking radon concentrations.

In a small fraction of houses with elevated radon the fluctuations over short time periods may vary by a factor of ten, sometimes reaching very high levels. Some of these houses have low radon concentrations for days followed by high radon concentrations for days. Houses exhibiting erratic levels most often have binary radon source terms - they are either on or off. Some examples are:

- High radon in well water results in spikes that occur during and immediately after water use showers, washing machines and baths (Scott 1988).

- Furnace or air conditioning ductwork that either pressurizes or depressurizes the soil beneath the slab produces radon spikes (Scott 1988).

- During times when the barometric pressure is dropping and there is heavy rainfall there is more resistance for the mass of air in the soil to equilibrate through the saturated soil surface and the amount soil air passing through the foundation of homes temporarily increase. This results in rain spikes of radon concentrations that have been reported in the literature. Rain spikes are infrequent enough that they probably do not have a significant impact on occupant exposure but they can have a significant impact on radon measurements made over the course of a few days.

- Houses that are situated on highly permeable soils, gravels or bedrock may allow wide variation in the concentration of entering soil air, depending on the geometry of the foundation, the nearby radium deposits and the direction of airflow through the soil. The best documented of these buildings are in Huntsville and Oak Ridge (Dudney 1992). Figure C.4 below shows the radon concentration in the basement of a house one third of the way up a ridge that rests on fractured limestone containing some caverns. The investigators suspect that differences in air density in the underground cavern and fissure network and outdoor air drive airflow through the limestone away from the house or toward the house. 


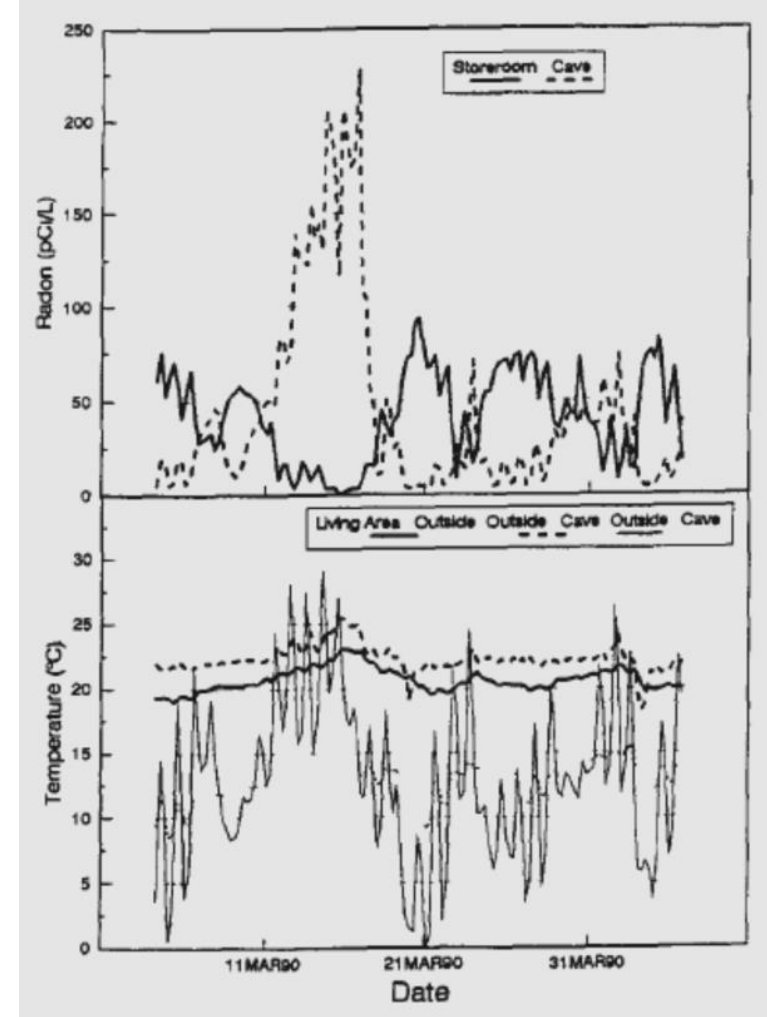

Fig. C.4 Wide variation in radon concentrations over short time periods (Dudney 1992).

\section{Radon Testing}

A variety of residential radon testing methods are utilized, some can be performed by homeowners themselves and others need to be performed by a radon testing professional. Radon measurement methods can be characterized by:

- $\quad$ how long they can be deployed (duration of sampling)

- whether the result will be a time integrated average radon concentration over the sampling time or whether the device can distinguish radon fluctuations over short time intervals as well as provide the average over the entire sampling time

- method of sample collection and analysis

- $\quad$ precision and bias errors 


\begin{tabular}{|c|c|c|c|c|}
\hline $\begin{array}{l}\text { Detector Type } \\
\text { (Abbreviation) }\end{array}$ & Passive/Active & $\begin{array}{c}\text { Typical Uncertainty } \\
{[\%]}\end{array}$ & $\begin{array}{c}\text { Typical Sampling } \\
\text { Period }\end{array}$ & Cost \\
\hline $\begin{array}{l}\text { Alpha-track Detector } \\
\text { (ATD) }\end{array}$ & Passive & $10-25$ & $1-12$ months & low \\
\hline $\begin{array}{l}\text { Activated Charcoal } \\
\text { Detector (ACD) }\end{array}$ & Passive & $10-30$ & 2 - 7 days & low \\
\hline $\begin{array}{l}\text { Electret Ion Chamber } \\
\text { (EIC) }\end{array}$ & Passive & $8-15$ & 5 days - 1 year & medium \\
\hline $\begin{array}{l}\text { Electronic Integrating } \\
\text { Device (EID) }\end{array}$ & Active & $\sim 25$ & 2 days - year(s) & medium \\
\hline $\begin{array}{l}\text { Continuous Radon } \\
\text { Monitor (CRM) }\end{array}$ & Active & $\sim 10$ & 1 hour - year(s) & high \\
\hline
\end{tabular}

Fig. C.5 Most commonly used radon measurement devices and their characteristics (WHO 2009).

The uncertainty listed in the table refers to precision error inherent in the devices and optimal exposure durations. Fluctuating radon concentrations are likely to be the largest source of error in measuring indoor radon to determine whether a building is greater than or equal to the EPA annual average action level of $4 \mathrm{pCi} / \mathrm{L}$.

As a consequence the effect of sampling duration requires special consideration. If the radon concentration in houses remained constant then a five minute grab sample would characterize the annual concentration. Cyclic changes in concentration require sampling over at least an entire and sampling over several would be better. Since there are diurnal cycles at least a 24 hour sample must be made. Because there are weekly fluctuations it is better to sample over the course of at least a week. Because there are seasonal variations it is better to measure over at least two seasons. Because the annual average is the desired outcome a yearlong radon measurement would provide the most confidence in the result.

If an annual average radon measurement is made there are three possible outcomes to the test:

- the building is clearly less than $4 \mathrm{pC} / \mathrm{L}$ annual average

- the building is clearly greater than $4 \mathrm{pCi} / \mathrm{L}$ annual average or

- it cannot be said whether the building is greater than or equal to or less than $4 \mathrm{pCi} / \mathrm{L}$ annual average because the result is within the uncertainty of the measurement.

Because it is an annual average the uncertainty due to fluctuating radon concentrations over time has been largely removed (reduced to year to year variation) and the confidence interval will be the smallest possible, thus minimizing the frequency of the "I can't tell whether it passes or fails" outcome.

For a number of reasons many people do not want to take the time to measure for a year to find out whether or not they have a problem. They may be nervous that the house has very high concentrations of radon and want to find out quickly. They may be selling or buying a house and find it is impractical to delay closing or put money in escrow. So they settle for a shorter test that has greater uncertainty in the result.

A number of studies have examined the effect of sample duration on the coefficient of variation of the measurement (Ronca-Batista 1988, Steck 2000). Figure C.6 below graphs the coefficient of variation for a grab sample plus continuous radon monitors used to sample for varying durations in 20 houses in 
Montana (Ronca-Batista 1988). Seventeen of these houses had winter peaking radon concentrations, largely due to opened windows. It is likely that during mild weather the houses had open windows because few houses in Montana were air conditioned before 1988. Three houses had neither summer nor winter peaking radon concentrations (EPA 1986). The test protocol did not require closed house conditions. The authors knew that one of the 3 non-peaking houses did not open windows during the summer but did not know the window status for the other two. The first data points are for five minute grab samples. Moving to one day measurements eliminates a great deal of variation. The top line includes hourly data for 12 months. The bottom line includes only closed house conditions - the winter months. Two things stand out:

- As duration of sample increases variation decreases.

- The coefficient of variation for winter months is around one third that for the annual average. Closed house conditions significantly reduce variability.

A 1 to 4 day sample duration may useful as a screening measurement, but has a great deal of uncertainty when comparing to an annual average that includes uncontrolled window opening during mild weather. For example, if the result comes back $2 \mathrm{pCi} / \mathrm{L}$ chances are good that the building has a low enough concentration that a long term follow up measurement could be completed without significant additional exposure. It the result comes back greater than 8 or $10 \mathrm{pCi} / \mathrm{L}$ chances are good that it exceeds the annual $4 \mathrm{pCi} / \mathrm{L}$ guideline.

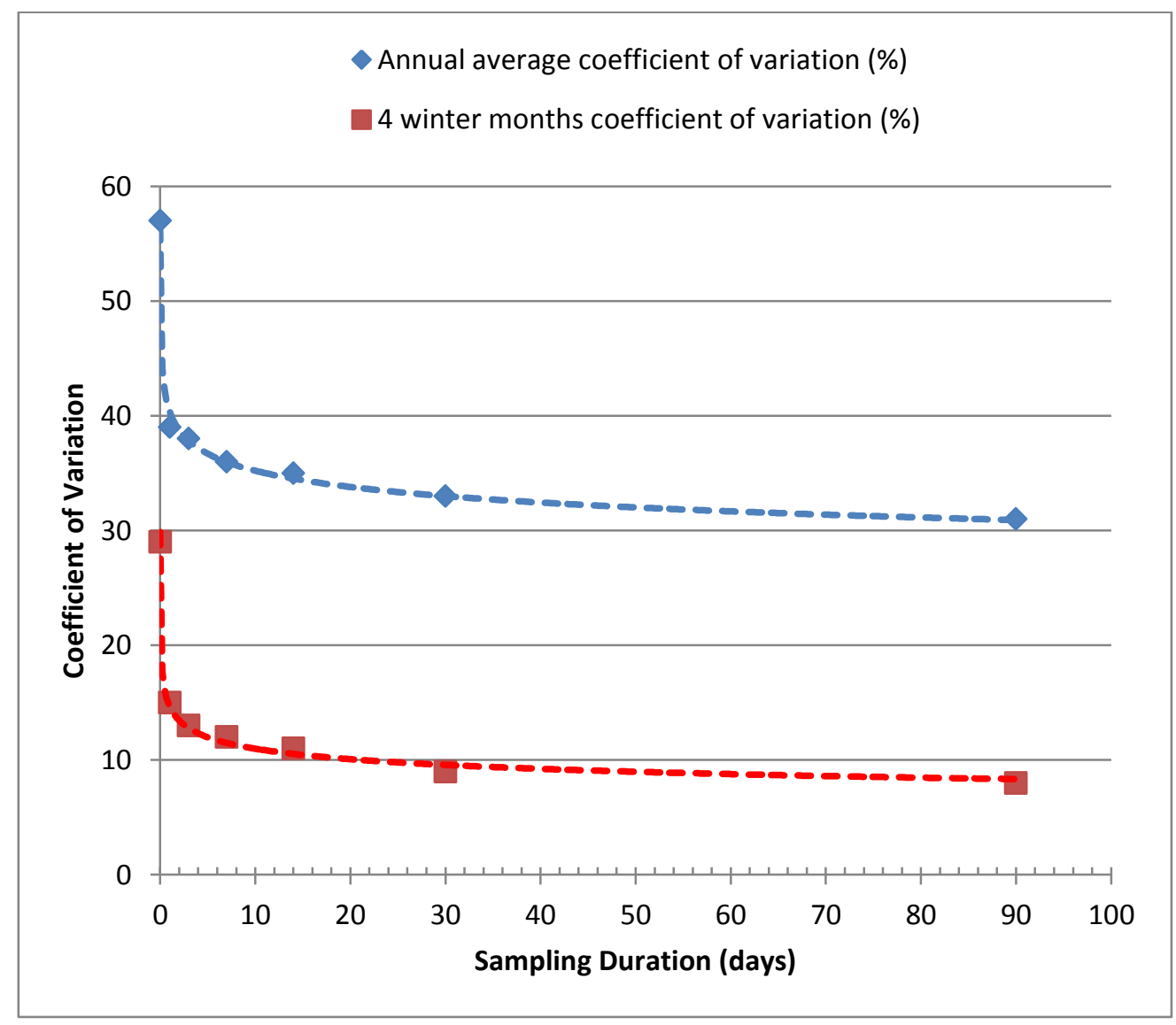

Fig. C.6 Coefficient of variation (\%) for varying duration of continuous radon monitor measurements compared to annual average and four winter months in Montana (Ronca-Batista 1988). 
The $4 \mathrm{pCi} / \mathrm{L}$ action level may lie within the uncertainty of the measurement. For example a short term result of $3 \mathrm{pCi} / \mathrm{L}$ may be obtained in a building with a $5 \mathrm{pCi} / \mathrm{L}$ annual average or a $5 \mathrm{pCi} / \mathrm{L}$ result may be returned for a building with an annual average of $3 \mathrm{pCi} / \mathrm{L}$.

Figure C.7 illustrates radon variability in a house with an average annual living space concentration of 2.7 pCi/L (Lewis 1999) but could easily have short term sample results that are close enough to $4 \mathrm{pCi} / \mathrm{L}$ to be within the uncertainty of the measurement. If short term measurements are made on the first floor in closed house conditions the result is likely to be near $4 \mathrm{pCi} / \mathrm{L}$ and within the uncertainty of the measurement. In this particular house the basement is radon level does not show a seasonal cycle.

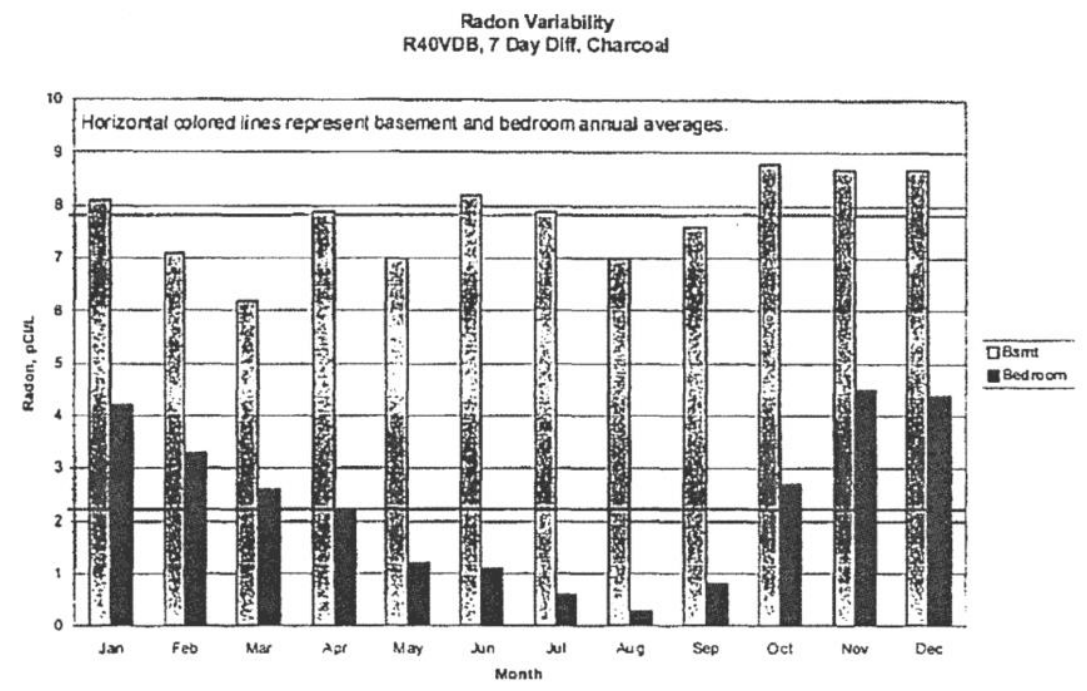

Fig. C.7 Monthly charcoal canister results for a single house in Pennsylvania (Lewis 1999).

Several studies have been conducted to determine the effect of sample duration on the coefficient of variation or likelihood of false negative and false positive results (Bierma 1989, Krewski 2005, Mose 1986, Roessler 1987, Ronca-Batista 1990, Steck 1988, Steck 2000, White 1990). Average winter to summer ratios for these studies ranged from 0.98 to 2.3 with most results in the 1.3 to 1.8 range. Reported values of false positive and false negative results are presented in the table below. 
Table C.2 False negative and positive when estimating annual averages based on 1 screening measurement.

\begin{tabular}{|c|c|}
\hline Bierma & $\begin{array}{l}\text { Screening methods alone (no followup measurements) found } 30 \%-36 \% \text { results } \\
\text { were false negative at } 4 \mathrm{pCi} / \mathrm{L}\end{array}$ \\
\hline Krewski 2005 & $\begin{array}{l}\text { Screening measurements found } 10 \% \text { false positive and false negative combined at } \\
4 \mathrm{pCi} / \mathrm{L}\end{array}$ \\
\hline Ronca-Batista 1990 & $\begin{array}{l}\text { Screening measurements found } 9 \% \text { false negatives for basement screening } \\
\text { location; } 35 \% \text { false negatives for first floor screening location (NOTE: this is a } \\
\text { product of higher basement than first floor levels) }\end{array}$ \\
\hline Steck 1988 & Screening measurements found $20 \%$ false negatives; $30 \%$ false positives \\
\hline Steck 1990 & $\begin{array}{l}78 \% \text { of true positives identified by screening test (95\% CI - 70\% - 85\%); } \\
93 \% \text { of true negative identified as negative by screening test (95\% CI 81\%-99\%) }\end{array}$ \\
\hline White 1990 & $\begin{array}{l}\text { In } 528 \text { houses } 354 \text { screening measurements were }<4 \mathrm{pCi} / \mathrm{L}, 11 \text { were false } \\
\text { negatives and } 174 \text { were } \geq 4 \mathrm{pCi} / \mathrm{L}, 84 \text { were false positives. }\end{array}$ \\
\hline
\end{tabular}

Steck (1988) presents the data in Fig. C.8 from a study of 80 houses in Minnesota in a graphical form making it easier to visualize false positives and false negatives.

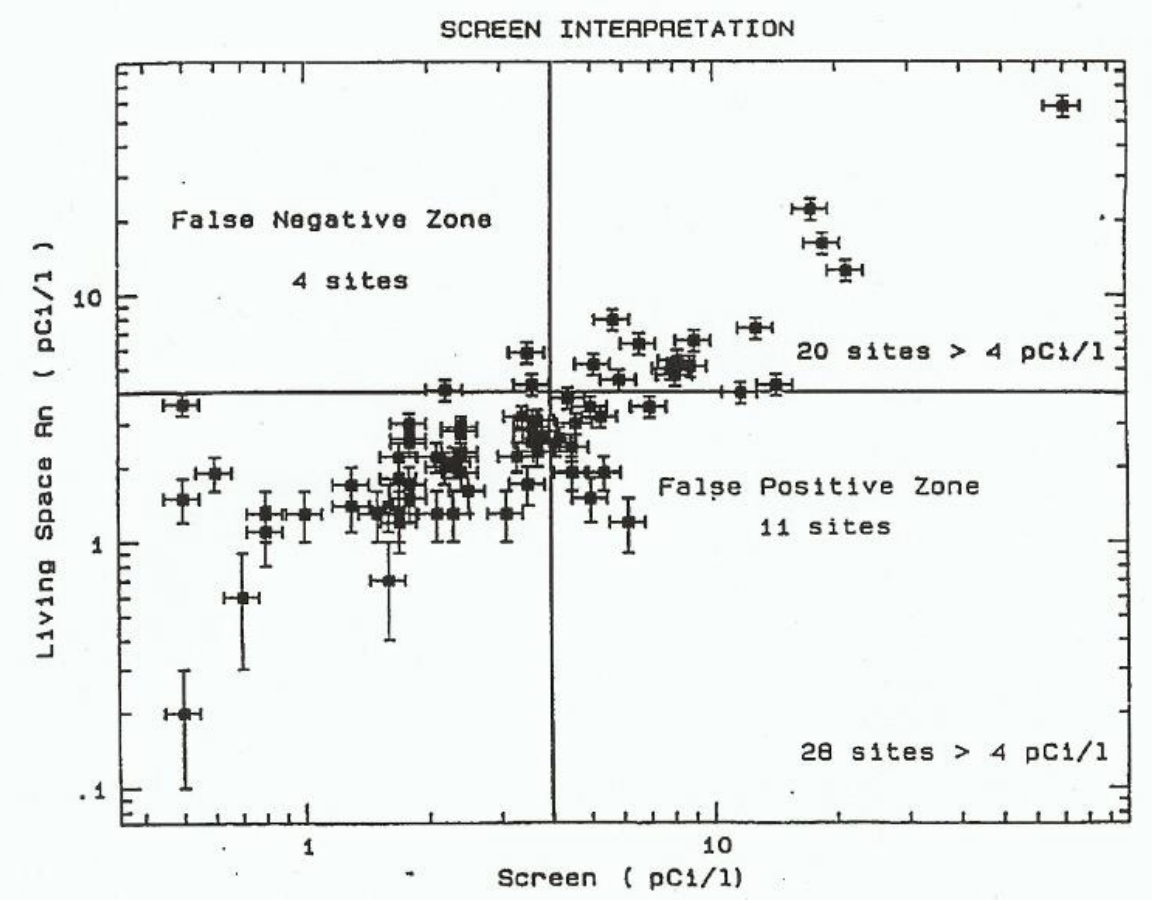

Fig. C.8 Screening measurements vs. annual average living space radon levels in 78 Minnesota houses (Steck 1990). 


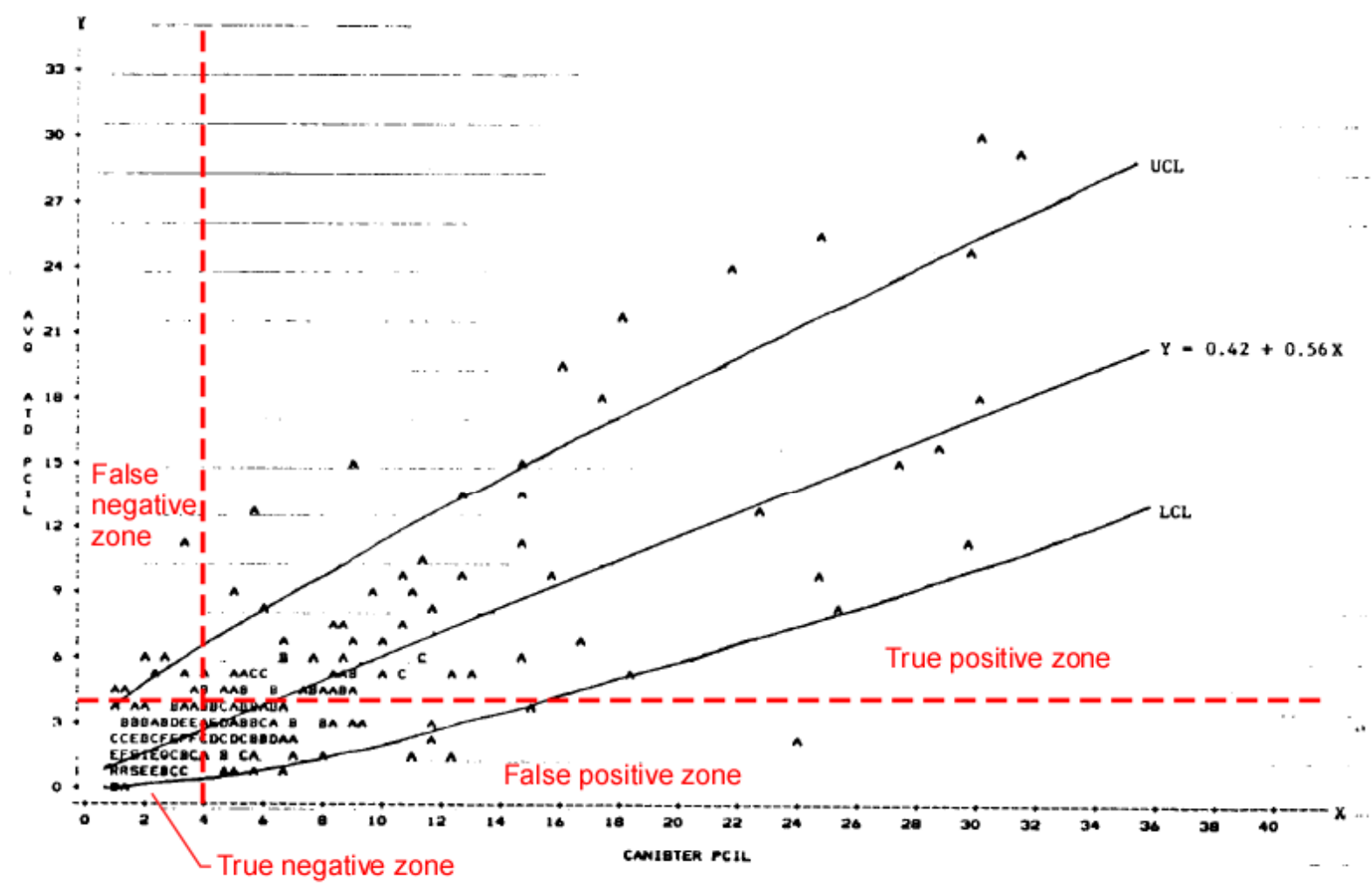

LEGEND: $A=10 B S, B=20 B S$, etc.

Fig. C.9 Markers at 4 pCi/L for screening and annual average measurements for 528 houses (White 1990).

Figure C. 9 above shows markers at $4 \mathrm{pCi} / \mathrm{L}$ for screening and annual average measurements from a much larger data set (528 houses) (White 1990). The upper and lower lines are the 95\% confidence interval. Subsets of the houses had additional screening measurements averaged with the first (See Fig. C.10 on next page); measurements are denoted by the letter $\mathrm{B}$ for two, $\mathrm{C}$ for 3, D for 4, etc. It is clear that additional measurements significantly reduce the scatter in the data. This is one of the few studies that examines the EPA protocol of screening measurement with a followup measurement if the screening measurement is greater than $4 \mathrm{pCi} / \mathrm{L}$. The datapoints farthest from the best fit line are all from single screening measurements. The datapoints with multiple screening measurement averages are shown in the figure below. All of the averaged screening results are closer to the line of best fit than the single tests. The datapoints that are an average of 4 or 5 short term tests are very close to the line. 


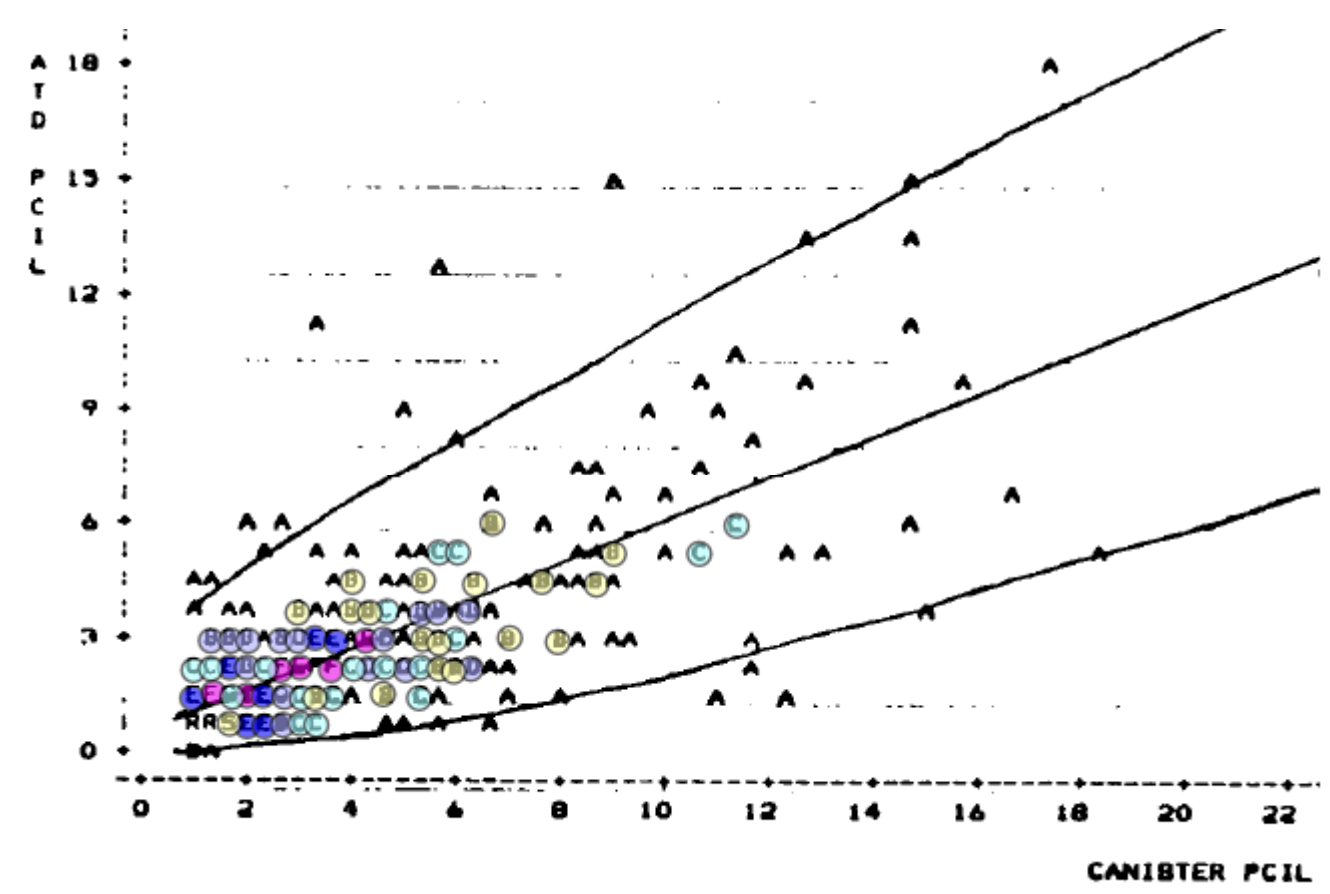

Fig. C.10 Subsets of homes (in Figure C.9) with additional screening measurements averaged with first measurements are denoted by the letter $B$ for two, $C$ for 3 , D for 4 , etc.

Multiple averaged short term tests greatly improve the statistics for estimating annual average from averaged shorter term tests. No false negatives occur while a significant number of false positives remain. This is a result of the slope of the line relating short term tests to annual average. Roesller (1987) reports similar improvements in confidence intervals when averaging multiple short term measurements.

It is clear that a sampling protocol must minimize the uncertainty due to duration of measurement. To address the fluctuations in radon measurements over time and also allow for the use of quick screening measurements the EPA (2005) recommends:

- Testing in closed house conditions on the lowest lived in level

- Making a short term screening measurement (between 2 and 90 days).

- The result of the screening measurement is used to determine whether to conduct a followup measurement or not.

- If the screening measurement is less than $4 \mathrm{pCi} / \mathrm{L}$ followup measurement is not recommended.

- If a screening measurement is greater than or equal to $4 \mathrm{pCi} / \mathrm{L}$ but less than $8 \mathrm{pCi} / \mathrm{L}$ the EPA recommends a long term measurement to better estimate the annual average.

If a screening measurement is greater than $8 \mathrm{pCi} / \mathrm{L}$ make a second short term measurement.

- Deciding whether to mitigate the radon in the house: 
- If a long term followup measurement is greater than or equal to $4 \mathrm{pCi} / \mathrm{L}$ mitigate the house

$\circ$ If the average of a screening and short term followup measurement is greater than or equal to $4 \mathrm{pCi} / \mathrm{L}$ mitigate the house.

Unfortunately only a few of the studies include screening and followup measurements in the datasets.

A national sample of annual living area average radon concentration (ALAA) measurements was collected in the National Residential Radon Survey (NRRS) in 1989-1990. This survey found the average ALAA level in U.S. homes to be 1.25 (+/- 0.12) pCi/L, although $6 \%$ of these homes (5.8 million) had radon levels greater than $4 \mathrm{pCi} / \mathrm{L}$ (Marcinowski 1994). Because of existing physical processes interacting in so many different ways, the extent of the relationship between radon concentrations and the tightening of a building envelope is ambiguous (Dyess 1994). To establish a true relationship, a multivariate model where these physical processes are statistically controlled should be considered (Chi and Laquatra 1990).

\section{Effects of Weatherization on Radon Levels}

Studies of the impact of weatherization and energy efficient construction radon levels can be divided into two categories: (1) studies involving pre- post-weatherization measurements of radon levels; and, (2) cross-sectional studies that compare radon levels between weatherized or-in the case of newly constructed homes-efficient homes and non-weatherized (or inefficient) homes.

\section{Pre/Post Studies}

In 1979, the Bonneville Power Administration assigned the task of measuring the effective leakage area in 12 houses in Richmond, WA to researchers from Lawrence Berkeley National Laboratory (LBNL) (Offermann 1981). This research was to assess the effectiveness of house tightening in reducing air leakage and also assist in a comparative evaluation of changes in indoor air quality pre- and post-retrofit work. The study examined the impact of additional air sealing in all-electric homes that had already received insulation and storm windows and doors. The average reduction in air leakage of $32 \%$ was accompanied by a $42 \%$ increase in short-term radon concentrations. The researchers concluded that "The moderate increases in radon...concentrations observed are consistent with what would be expected from the estimated average decrease in air-exchange rate," but also noted that uncertainties remained and that a definitive conclusion could not be made that the observed increase in radon levels was due to the retrofits.

A 1981 report from LBNL on the impact of retrofits on indoor air quality on nine all-electric houses in the Medford, Oregon area concluded that the impact of the retrofits installed in the homes on air quality appeared to be minimal, but noted that impacts depend heavily on the retrofit measures completed and the type of heating and cooling system in the home. However, detailed measurements of pollutants, including radon, were made in only two homes, both of which had pre-existing radon levels at or near the detectable limit.

In 1988, LBNL reported on a study involving 40 treatment and 8 control homes in Eastern Washington and Northern Idaho. Thirty-five homes received a standard weatherization package, and five received "house doctoring". The standard package resulted in an air infiltration reduction of approximately 13\%; while house doctoring resulted in a reduction in leakage of $26 \%$. Pre-weatherization, the mean radon levels in one area was $2.6 \mathrm{pCi} / \mathrm{L}$, which was due to the local highly-permeable, gravelly soils, and the other study area measured a mean radon level of $1.1 \mathrm{pCi} / \mathrm{L}$. Of these two areas, $27 \%$ exceeded $4 \mathrm{pCi} / \mathrm{L}$. Post-weatherization, among the homes that had crawlspaces, an average decrease of $33 \%$ in radon concentrations was observed, which was attributed to the installation of mechanical ventilation within the 
crawlspaces. Reductions were seen in homes with other types of substructure but at a statistically insignificant level (Grimsrud 1988).

In 1994, EPA reported on a 1990-91 project with the Maryland Weatherization Assistance Program in which 4- to 6-week radon measurements were made before and after weatherization work was completed (Dyess 1994). The study involved 32 homes that received "lesser" weatherization treatment, 28 homes that received "advanced" weatherization and 16 homes that served as unweatherized controls during the study period. The homes receiving the standard weatherization package had air leakage reductions on the order of 10 to 15 percent, while those receiving the advanced package had 35 to 40 percent reductions. Post-weatherization data showed that the indoor radon concentrations decreased with statistical significance for the group of homes that received the less rigorous treatment as well as the control group (See Fig. C.11). For the group of homes receiving the more rigorous treatment, the radon levels rose slightly but were not considered statistically significant compared to the level of reduction in air leakage. However, these interpretations were somewhat clouded due to inconsistencies in the existing weather conditions present between all three groups. For the control group, outdoor temperature and precipitation levels changed significantly between the two measurement phases. For the rigorously treated group, only outdoor temperature varied, while the other group receiving lesser air reduction measures experienced no significant change in either.

\begin{tabular}{|lc|}
\hline $\begin{array}{l}\text { Measurement } \\
\text { Parameter }\end{array}$ & $\begin{array}{c}\text { Difference (Ave.) } \\
\text { Pre- Post- Values }\end{array}$ \\
\hline Advanced Weatherization & \\
Indoor Radon, pCi/L & 0.4 \\
Precipitation & -0.8 \\
Outdoor Temperature & -6.7 \\
Airtightness, ACH @ 50 Pa & -9.1 \\
& \\
Lesser Weatherization & \\
Indoor Radon, pCi/L & -0.3 \\
Precipitation & -0.4 \\
Outdoor Temperature & -3.8 \\
Airtightness, ACH @ $50 \mathrm{~Pa}$ & 2.8 \\
& \\
No weatherization (Control) & \\
Indoor Radon, pCi/L & -0.3 \\
Precipitation & 2.9 \\
Outdoor Temperature & -5.6 \\
Airtightness, ACH @ $50 \mathrm{~Pa}$ & -0.5 \\
& \\
\hline
\end{tabular}

Fig. C.11 Summary of Monitoring Results by Type of Weatherization Procedure. (Dyess 1994) 


\section{Cross-Sectional Studies}

Fleischer reported on a cross-sectional study of annual radon levels in 27 homes in New York (Fleischer 1982), of which 14 were categorized as efficient and 13 were described as inefficient based on the owners' descriptions. Fleischer concludes that "the causes of the elevated radon are diverse; however, the chief reason is that airtight homes retain radon longer than well-ventilated ones and hence allow buildup to higher levels." However, a later cross-sectional study of 245 homes in New York (Chi and Laquatra 1990) found no relationship between short-term radon levels and degree of weatherization. Both of these studies relied on survey self-reports of weatherization activities (such as caulking and weatherstripping, the installation of insulation and the installation of storm windows and doors), did not involve direct measurements of air leakage, and did not examine radon levels before and after installation of weatherization measures in homes.

Turk et al. (1987) report on a cross-sectional study of new homes in the Pacific Northwest involving 29 homes built to model conservation standards (MCS) and 32 control homes that did not adhere to these standards. The MCS homes exhibited nearly 50 percent less air leakage, but-owing to the installation of heat-recovery ventilation equipment-exhibited similar actual air exchange rates (based on tracer gas tests) as the control homes. The study found that geographic location was the most important determinant of radon levels, and did not find any significant differences in radon levels associated with construction practice.

The table below summarizes the relevant studies related to radon and weatherization. 
Table C.3. Summary of relevant studies related to radon and weatherization.

\begin{tabular}{|c|c|c|c|c|c|c|c|c|c|}
\hline Study & Year & $\begin{array}{l}\text { Studied } \\
\text { WAP } \\
\text { homes? }\end{array}$ & Approach & $\begin{array}{l}\text { Geographic } \\
\text { scope }\end{array}$ & Sample size & $\begin{array}{c}\text { Radon } \\
\text { measurement } \\
\text { length and } \\
\text { season }\end{array}$ & $\begin{array}{l}\text { WX air } \\
\text { leakage } \\
\text { reduction }\end{array}$ & Key conclusion & Notes \\
\hline Offerman & 1981 & No & pre/post & Washington & 12 & $\begin{array}{l}14 \text { days in } \\
\text { November } \\
\text { (pre) and } \\
\text { January (post) }\end{array}$ & $32 \%$ & $\begin{array}{l}\text { "The moderate increases in } \\
\text { radon...concentrations } \\
\text { observed are consistent with } \\
\text { what would be expected from } \\
\text { the estimated average } \\
\text { decrease in air-exchange } \\
\text { rates...Uncertainties } \\
\text { remain..." }\end{array}$ & $\begin{array}{l}\text { Study was of the } \\
\text { impact of additional } \\
\text { tightening of homes } \\
\text { that had already } \\
\text { received insulation } \\
\text { and storm } \\
\text { windows/doors. } \\
\text { Homes were all- } \\
\text { electric. }\end{array}$ \\
\hline Berk & 1981 & No & pre/post & Oregon & 2 & 14 days & $20-40 \%$ & $\begin{array}{l}\text { "The impact on indoor air } \\
\text { quality of the energy- } \\
\text { conserving retrofits....appears } \\
\text { to be minimal...On the other } \\
\text { hand, the potential for } \\
\text { reducing air leakage has not } \\
\text { been fully realized." }\end{array}$ & $\begin{array}{l}\text { Study involved } 9 \\
\text { homes, but radon } \\
\text { measurements were } \\
\text { made in two. } \\
\text { Homes were all- } \\
\text { electric. Pre- } \\
\text { treatment radon } \\
\text { levels were }<1 \mathrm{pCi} / \mathrm{L} \\
\text { in both homes. } \\
\end{array}$ \\
\hline $\begin{array}{l}\text { Fleischer } \\
\text { et al. }\end{array}$ & 1982 & No & $\begin{array}{l}\text { cross- } \\
\text { sectional }\end{array}$ & New York & 27 & one year & unknown & $\begin{array}{l}\text { "The causes of the elevated } \\
\text { radon are diverse; however, } \\
\text { the chief reason is that airtight } \\
\text { homes retain radon longer } \\
\text { than well-ventilated ones and } \\
\text { hence allow buildup to higher } \\
\text { levels." }\end{array}$ & $\begin{array}{l}\text { Delineation of } \\
\text { energy-efficient } \\
\text { homes }(n=14) \text { versus } \\
\text { non-efficient }(n=13) \\
\text { based on owner's } \\
\text { description. }\end{array}$ \\
\hline
\end{tabular}




\begin{tabular}{|c|c|c|c|c|c|c|c|c|c|}
\hline Study & Year & $\begin{array}{l}\text { Studied } \\
\text { WAP } \\
\text { homes? }\end{array}$ & Approach & $\begin{array}{c}\text { Geographic } \\
\text { scope }\end{array}$ & Sample size & $\begin{array}{c}\text { Radon } \\
\text { measurement } \\
\text { length and } \\
\text { season }\end{array}$ & $\begin{array}{c}\text { WX air } \\
\text { leakage } \\
\text { reduction }\end{array}$ & Key conclusion & Notes \\
\hline $\begin{array}{l}\text { Turk et } \\
\text { al. }\end{array}$ & 1987 & No & $\begin{array}{l}\text { cross- } \\
\text { sectional }\end{array}$ & $\begin{array}{l}\text { Washington, } \\
\text { Oregon, } \\
\text { Idaho }\end{array}$ & $\begin{array}{l}29 \text { new } \\
\text { homes built to } \\
\text { model } \\
\text { conservation } \\
\text { (MCS) stds.; } \\
32 \text { controls }\end{array}$ & 55-70 days & $\begin{array}{l}\text { MCS homes } \\
\text { averaged } \\
46 \% \text { lower } \\
\text { air leakage; } \\
\text { no difference } \\
\text { in measured } \\
\text { actual } \\
\text { ventilation } \\
\text { rate }\end{array}$ & $\begin{array}{l}\text { "In general, indoor } \\
\text { concentrations of } \\
\text { radon...exhibited greater } \\
\text { dependence on the region in } \\
\text { which a house was located } \\
\text { than on the constructions } \\
\text { practices by which it was } \\
\text { built. Differences in radon } \\
\text { levels between MCS and } \\
\text { Control houses by region or } \\
\text { for all houses are not } \\
\text { considered significant." }\end{array}$ & $\begin{array}{l}\text { Study of new homes, } \\
\text { rather than } \\
\text { weatherization of } \\
\text { existing homes. } \\
\text { MCS homes had } \\
\text { heat recovery } \\
\text { ventilation } \\
\text { equipment. }\end{array}$ \\
\hline Grimsrud & 1988 & No & $\begin{array}{l}\text { pre/post } \\
\text { w/ control }\end{array}$ & $\begin{array}{l}\text { Washington, } \\
\text { Idaho }\end{array}$ & $\begin{array}{l}40 \text { treatment } \\
\text { (three } \\
\text { groups); } 8 \\
\text { control }\end{array}$ & $\begin{array}{c}\text { 55-70 days, } \\
\text { season not } \\
\text { specified }\end{array}$ & $\begin{array}{l}12.5 \% \text { for std } \\
\text { wx }(\mathrm{n}=35) ; \\
38 \% \text { for } \\
\text { "house } \\
\text { doctoring" } \\
\text { homes }(\mathrm{n}=5)\end{array}$ & $\begin{array}{l}\text { "Radon concentrations } \\
\text { decreased in crawlspace } \\
\text { houses due to weatherization. } \\
\text { Reductions in house with } \\
\text { other foundation types were } \\
\text { seen but were not statistically } \\
\text { significant." }\end{array}$ & $\begin{array}{l}\text { Observed radon } \\
\text { concentration } \\
\text { reduction in } \\
\text { crawlspace homes } \\
\text { was attributed to } \\
\text { added ventilation. }\end{array}$ \\
\hline $\begin{array}{l}\text { Chi and } \\
\text { Laquatra }\end{array}$ & 1990 & No & $\begin{array}{l}\text { cross- } \\
\text { sectional }\end{array}$ & New York & 245 & $\begin{array}{l}\text { 3-7 days under } \\
\text { closed-house } \\
\text { conditions }\end{array}$ & unknown & $\begin{array}{l}\text { "...there is no significant } \\
\text { relation between the degree of } \\
\text { weatherization and radon } \\
\text { levels in the house..." }\end{array}$ & $\begin{array}{l}\text { Study relied on } \\
\text { survey self-reports } \\
\text { of a limited checklist } \\
\text { of weatherization } \\
\text { activities (caulking } \\
\text { and } \\
\text { weatherstripping, } \\
\text { storm windows and } \\
\text { doors, insulation). }\end{array}$ \\
\hline Dyess & 1990 & Yes & $\begin{array}{l}\text { pre/post } \\
\text { w/ control }\end{array}$ & Maryland & $\begin{array}{c}60 \text { treatment } \\
\text { (two-groups); } \\
16 \text { control }\end{array}$ & $\begin{array}{l}\text { 4-6 weeks } \\
\text { during the } \\
\text { heating season }\end{array}$ & $\begin{array}{l}10-15 \% \text { std- } \\
\text { wx homes } \\
(\mathrm{n}=32) ; 35- \\
40 \% \text { for } \\
\text { "advanced" } \\
\text { wx homes } \\
(\mathrm{n}=28)\end{array}$ & $\begin{array}{l}\text { "Weatherization activities did } \\
\text { not adversely affect radon } \\
\text { levels. However, } \\
\text { interpretations are clouded by } \\
\text { weather factors..." }\end{array}$ & \\
\hline
\end{tabular}




\section{Radon Remediation}

The first remediation efforts by EPA began in the early 1970's in Grand Junction, Colorado, where uranium mine tailings had been used as fill under new construction and/or an aggregate material for concrete or masonry. Removal of contaminated materials was the preferred method of addressing radon contamination at that time, but due to economic considerations soil remediation became the new approach (Angell 2008). Seamless epoxy coatings were used as barriers due to the thought that radon diffusion through these materials was the primary method of exposure. In the late 1970's, due to further investigation, an active soil depressurization technique was developed. Experts found that interior drain tiles $^{38}$ and sump pumps were the primary source of entry rather than diffusion from soil or building materials; therefore they stated that attention needed to be on methods of reducing soil gas infiltration rather than barrier coatings (Angell 2008).

Over the years, several radon control techniques have been developed, tested and implemented. However, insufficient amounts of large-scale randomized studies have been yet to be completed on the impact of remediation on radon levels (Henschel 1993). In the early 1980's, the infiltration-reduction became the recommended mitigation method. Then by 1994, the conventional and most common remediation method was the active sub-slab depressurization (ASD) (Henschel 1993). With ASD, soil gas is exhausted to the outside through pipes running through the slab floor with the use of electric fans. Laying gravel under the slab floor increases ASD efficacy (Fisk 1994). Numerous studies have shown that ASD is very successful. LBNL conducted detailed systematic studies of radon mitigation using a number of methods in the Spokane River Valley in the late 1980s (Turk 1987). The EPA Office of Research and Development (ORD) conducted demonstration projects in New Jersey, Pennsylvania, Tennessee and Florida. New York State Energy Research and Development Authority (NYSERDA) conducted a number of well-designed studies in New York State that included a number of mitigation techniques in addition to ASD. ASD works in almost all houses tested. The two only major barriers was when the substrate consisted of highly air permeable materials - e.g. glacial till, shattered shale, karst or limestone. The depressurizing fans could draw air through these substrates so easily that they operated at the full flow-low pressure end of the fan curve and only weak low pressure was induced beneath the slab. Connecting more suction points to a single fan or pressurizing beneath the slab provided effective control in these cases. Although, the installation cost run from $\$ 1000-\$ 1500$ and uses a substantial amount of energy from the electric fans. ASD systems also increase heating and cooling costs due to increased ventilation; the net present value of this system is about $\$ 2000$. There is a slightly different mitigation measure called the active sub slab pressurization (ASP), which pulls pressurized air into the sub slab area reversing the air flow which forces the radon gas down into the soil. An electric fan is used in this case as well and again results in increased ventilation rates that could increase heating and cooling costs. Longterm reliability of these two types of systems is a concern due to failed fans and occupants turning off fans from noise disruption and energy use (Fisk 1994).

In 1994, Fisk evaluated two mitigation methods referred to as the membrane and short-circuit technique (MASC) and the efficient active sub slab pressurization technique (EASP). The MASC entails a combination of installation of a diffusion "membrane", several sheets of plastic on top of the soil and below the sub slab gravel, and sections of insulated pipe called "short-circuit" pipes that extend between sub slab gravel and outdoors. In a paper by Bonnefous et al. (1993), it was stated that the membrane significantly reduces the rate of diffusion as well as pressure driven radon flow from soil into gravel (cited in Fisk). Depending on soil permeability, these layers of plastic sheeting placed beneath sub-slab gravel will reduce radon entry rates (Fleischer, Mogro-Campero, \& Turner, 1982) by 10-50\%. As part of this evaluation, a lab experiment was completed to measure the amount of radon that would diffuse

\footnotetext{
${ }^{38}$ Drain tiles are perforated, corrugated plastic pipes laid at the bottom of a foundation wall, used to drain excess water away from the foundation.
} 
through these plastic membranes. The diffusion rate through a $140 \mathrm{~m}^{2}$ membrane, with the radon concentration within the soil being $1000 \mathrm{pCi} / \mathrm{L}$ was approximately at $2.7 \mathrm{pCi}$ per second. If the house had a ventilation rate of $.4 \mathrm{ACH}$ (air changes per hour), the resulting indoor radon concentration would be $0.05 \mathrm{pCi} / \mathrm{L}$ (Fisk 1994). These methods are not useful for existing buildings. Placing water and water vapor impermeable beneath a drainage layer that includes fines in the gravel or consists entirely of sand has been implicated in slab moisture problems in the forensic literature.

In 2009, the most effective strategies still focused on sealing the radon entry routes along with ASD method (WHO 2009). A combination of strategies is recommended for increased efficacy (Zeeb 2009). Radon levels can be mitigated by several means but the lowest cost measure is the diffusion barrier.

\section{REFERENCES}

Akerblom, G. (1999). Radon Legislation and National Guidelines. SSI Report 99:18, Swedish Radiation Institute, July 1999.

Angell, W. J. (2008). The U.S. Radon Problem, Policy, Program and Industry: Achievements, Challenges and Strategies. Radiation Protection Dosimetry, Vol. 130, No. 1, 8-13.

Arvela, H. (1995). Seasonal Variation in Radon Concentration of 3000 Dwellings with Model Comparisons. Radiation Protection Dosimetry, 33-42.

Berk, J. V. (1981). Impact of Energy-Conserving Retrofits on Indoor Air Quality in Residential Housing. Berkeley: Lawrence Berkely National Laboratory.

Belanger, W. (1990). Prediction of Long-Term Average Radon Concentrations in Houses Based on ShortTerm Measurements. U.S. Environmental Protection Agency.

Bierma, T. (1989). Accuracy and Precision of Home Radon Monitoring and Effectiveness of EPA Monitoring Guidelines. JAPCA, Vol. 39, No.7, 953-959.

Borak, T. (1989). A Survey of Winter, Summer and Annual Average Radon Concentrations in Family Dwellings. Health Physics, Vol. 57, No. 3, 465-470.

Chi, P. S., and Laquatra, J. (1990). Energy Efficiency and Radon Risks in Residential Housing. Energy Vol. 15, No. 2, 81-89.

Chia-yu Lin, A. G. (1999). Analysis of Local Decisions Using Hierarchical Modeling, Applied to Home Radon Measurement and Remediation. Statistical Science, Vol. 14, No. 3, 305-328.

Darby S, et al. (2005). Radon in Houses and Risk Of Lung Cancer: Collaborative Analysis of Individual Data from 13 European Case-Control Studies. British Medical Journal, Vol. 330: 218-223.

Darby S., et al. (2006). Indoor Radon and Lung Cancer. Epidemiology. Vol. 17:121-122.

Dudney, C., et al. (1989). Indoor Radon in Tennessee Valley Houses: Seasonal, Building, and Geological Factors. Oak Ridge, TN: Oak Ridge National Laboratory.

Dyess, T. M. (1994). Assessment of the Effects of Weatherization on Residential Radon Levels. U.S. Environmental Protection Agency.

Environmental Protection Agency (1987). Radon Reference Manual. Office of Radiation Programs, Washington, D.C.

Fisk, W. J. (1994). New Methods of Energy Efficient Radon Mitigation. Berkeley: Lawrence Berkeley National Laboratory. 
Fleischer, R. L., Mogro-Campero, A., \& Turner, L. G. (1982). Indoor Radon Levels: Effects of EnergyEfficiency in Homes. Environment International, 105-109.

Grimsrud, D. (1988). The Compatibility of Energy Conservation and Indoor Air Quality. Berkeley: Lawrence Berkeley National Lab.

Hans, Jr., J. and Lyon, R. (1986). Seasonal Variations of Radon and Radon Decay Product Concentrations in Single Family Homes. Office of Radiations Programs, Las Vegas: U.S. Environmental Protection Agency.

Henschel, D. B. (1993). Radon Reduction Techniques for Existing Detached Houses: Technical Guidance (Third Edition) for Active Soil Depressurization Systems. Research Triangle Park: Environmental Protection Agency.

Hull, D. and Reddy, T.A. (1989). Study on the Reliability of Short-Term Measurements to Predict LongTerm Basement Radon Levels in a Residence. Princeton, NJ: Center for Energy and Environmental Studies.

Krewski, D., et. al. (2005). Modeling Seasonal Variation in Indoor Radon Concentrations. Journal of Exposure Analysis and Environmental Epidemiology, 2324-243.

Lewis, R. (1999). Measurement of Radon Variability in a Single Family Home under Normal Living Conditions. Harrisburg, Pennsylvania: Bureau of Radiation Protection/Radon Division.

Lubin, J.H., et al. (2004). Risk of Lung Cancer and Residential Radon in China: Pooled Results of Two Studies. International J of Cancer. Vol. 109:132-137

Marcinowski, F. E. (1994). National and Regional Distributions of Airborne Radon Concentrations in U.S. Homes. The Radiation Safety Journal: Health Physics, Volume 6; Issue 6.

Mose, D., et. al. (1986). Realistic Uncertainties for Charcoal and Alpha-Track Radon Monitors. Fairfax, Virginia: George Mason University.

Offermann, F. J. (1981). Midway House-Tightening Project: A Study of Indoor Air Quality. California: Lawrence Berkeley National Laboratory.

Roessler, C.E. (1987). Temporal Patterns of Indoor Radon in North Central Florida and Comparison of Short-Term Monitoring to Long-Term Averages. Gainesville: University of Florida.

Ronca-Battista, M. and Magno, P. (1988). A Comparison of the Variability of Different Techniques and Sampling Periods for Measuring Radon and its Decay Products. Health Physics, Vol. 55, No. 5, 801-807.

Ronca-Batista, M. (1990). The Relationship between Winter Screening and Annual Average Radon Concentration in U.S. Homes. Virginia: Scientific and Commercial Systems Corporations.

Scott, A. G., (1988). Effect of Indoor Radon Variability on the Duration and Interpretation of Radon Measurements. Wilmington, Delaware: American Atcon Inc.

Scott, A. G., (1993). Indoor Radon Concentration Variability and Radon Measurement Variations. Mississauga, Ontario: American Atcon Inc.

Steck, D. J., (1988). Statewide Radon Surveys: Screening vs. Long-Term Measurements. Collegeville, Minnesota: St. John's University. 
Steck, D. J., e. al. (2000). Indoor Radon Exposure Uncertainties Caused by Temporal Variation. Collegeville, Minnesota: St. John's University.

Synnott, F. (2005). An evaluation of Radon reference levels and Radon measurement techniques and protocols in European countries. Radiological Protection Institute of Ireland.

Turk, B. H., et al. (1987). A Comparison of Indoor Air Quality in Conventional and Model Conservation Standard New Homes in the Pacific Northwest: Final Report (LBL-23429). California: Lawrence Berkeley National Laboratory.

Turk, B.H., et al. (1988). Intensive Radon Mitigation Research: Lessons Learned. California: Lawrence Berkeley National Laboratory.

White, S. B., et al. (1990). A Statistical Analysis: Predicting Annual Radon Concentrations from 2-Day Screening Tests. North Carolina: Research Triangle Institute.

Zeeb, H. E. ed. (2009). WHO Handbook On Indoor Radon: A Public Health Perspective. Geneva: World Health Organization. 
APPENDIX D. FIELDWORK TRAINING AND PROTOCOL 



\section{APPENDIX D. FIELDWORK TRAINING AND PROTOCOL}

This appendix provides a more detailed description of the training that field technicians received and the protocols that they followed in conducting the study. We distinguish here between two project staff functions for the fieldwork. Field technicians had existing skills related to home performance testing, and implemented the main field protocol for the study. Pick-up technicians visited homes about a week after each field technician visit. Their primary responsibility was to retrieve the one-week radon and formaldehyde samplers that had been deployed; however, they also provided additional assistance as described below.

Field technicians -

- deployed samplers and data loggers;

- completed combustion safety, air leakage and other on-site test; and,

- completed a detailed data form regarding various characteristics of the sites

\section{Pick-up technicians -}

- verified that radon and formaldehyde samplers had been properly deployed, then sealed, retrieved and shipped samplers to the test labs;

- $\quad$ verified the operation of installed data loggers;

- retrieved refrigerator electricity loggers (which were deployed for an unrelated aspect of the overall evaluation)

- recorded additional site information regarding secondary refrigerators and freezers in the home; and,

- assisted households in completing the occupant survey.

Each site received four visits: a Phase A (pre-weatherization) visit by a field technician and a pick-up technician, and a Phase B (post-weatherization) visit by each.

\section{Field Technicians}

\section{Training Sessions}

All field technicians were provided with a two-day training prior to deployment in the field. Each training session involved a maximum of 8 trainees provided by two trainers. The first day of the training was conducted in a classroom, and covered all aspects of the field equipment, protocols and form. The second day involved practicing the protocols in one or more test homes. Additionally, field technicians were monitored by a member of the study technical team during their first week of deployment: this provided an opportunity to correct errors, as well as for the technician to ask questions and receive additional oneon-one training.

The classroom component consisted of several parts. An introduction to the overall project was provided, including the chain of command and the roles of the various entities involved. This introduction also included a brief discussion of the intent of the overall project and the other studies within the project, such as energy evaluation and process evaluation.

Two screens were used simultaneously during the classroom portion. On one a PowerPoint slideshow was presented. On the other was the data collection form to be used in the field. The slideshow included 
information on the IAQ samplers that were to be deployed, the methods for evaluating combustion appliances, and the methods for additional diagnostics of parameters considered potentially relevant to IAQ such as house airtightness, duct leakage, and pressurization due to air handler operation.

Additionally, samples of each type of IAQ measurement device were passed around when the device was introduced. Instructions for filling out IAQ sampler forms for submission to labs were also provided, with photos showing the forms filled out. This was pertinent for radon and formaldehyde.

Pictures of IAQ sampler deployments, both from a distance and up close, were shown to provide a context in which to locate samplers. The attendees were instructed to take similar photos to allow for pickup field staff to easily find the samplers and for post-weatherization field technicians to locate the post-weatherization samplers in the same locations.

Following the classroom portion of the training, the attendees were taken to a home on which to perform the protocol. The home was recruited by the instructors, sometimes in cooperation with a local weatherization agency, and was selected to provide characteristics amenable to performing the required tests in the protocol. All IAQ samplers were deployed and all forms were filled out. Discussions of how to address various conditions were had to ensure understanding by the attendees. When time allowed, a short visit to a second home with different characteristics (e.g. different type of heating appliance) was made.

Field technicians were also provided with a binder that included all background information, copies of the client leave-behind (explaining the purposes of the study, what was expected of them, and what they would receive), an equipment list, and an incentive payment receipt that was to be signed by the client.

\section{Field Data Collection Protocol}

The first portion of the data collection form included several sections regarding basic characteristics of the home. These included basic occupant information, house characteristics (e.g. house type, and area and volume for foundations and main living levels), hot water temperature at the kitchen sink (after temperature stabilizes, 3 minutes max), detailed characteristics of foundations (e.g. wall type, floor type, ducts in space, exposed dirt), and sections for a qualitative moisture assessment of foundations and abovegrade areas.

For the moisture assessment sections, the following issues were targeted:

- Window condensation in above-grade spaces $(\mathrm{Y} / \mathrm{N})$

- Standing water in foundations $(\mathrm{Y} / \mathrm{N})$

- Water stains in foundations and above-grade spaces (0-3 scale depending on size of affected area)

- Mold in foundations and above-grade spaces (0-3 scale depending on size of affected area)

- Musty smell in foundations and above-grade spaces (Y/N)

Following this section was the section on IAQ samplers. These samplers included

- radon canisters, deployed for 1 week (each home)

- formaldehyde badges, deployed for 1 week (2 homes per area)

- passive carbon monoxide loggers, deployed for 1 week (each home)

- temperature/relative humidity loggers, deployed for study duration (each home)

- temperature loggers (for unvented combustion appliances), deployed for 1 week (2 homes per area)

- refrigerator temperature, in place during site visit

- refrigerator energy loggers, deployed for 1 week (each home as accessible) 
The location of the sampler deployment was a major focus of the training and protocol. For radon, formaldehyde, and carbon monoxide, the samplers were located in an open area so as not to impede air circulation from reaching the samplers, but also away from exterior windows and walls, not in kitchens or bathrooms, approximately at breathing height (not on the floor or at the ceiling), and where they were unlikely to be disturbed by people or pets. Radon canisters were deployed both on the first floor and in any accessible crawl space or basement. In general, canisters were not placed under mobile homes, though field technicians were given discretion to place canisters beneath mobile homes when the crawl space perimeter was unusually tight.

Temperature/relative humidity loggers were placed on the central thermostat whenever possible. In the event that this location was on an exterior wall or was likely to be disturbed then a different central location within the home was selected. Temperature loggers (with thermocouple probes) for unvented combustion appliances were deployed when there was an expectation of frequent appliance use. These loggers were intended to be indicators of appliance use; the actual temperature was not of substantial interest. The priority for these temperature loggers was devices that might produce carbon monoxide; i.e., unvented heating appliances and ovens that were used frequently. Refrigerator temperature loggers were placed in the refrigerator at the beginning of each house visit and removed upon departure. Refrigerator energy loggers were installed whenever the outlet could be accessed readily and when the placement of the logger would not make recovery difficult.

The next section of the protocol related to space conditioning and water heating appliances, with a special focus on combustion appliances. Basic characteristics of heating, air conditioning, and hot water systems were recorded (e.g. type of venting, fuel type, location). Combustion testing was performed on all applicable appliances as follows:

- basic investigation for significant gas leaks

- measurement of $\mathrm{CO}$ in ovens (after 5 minutes) and 6 inches over each range top burner (after 3 minutes)

- measurement of $\mathrm{CO}$ in unvented heating appliances (after 3 minutes)

- determination of worst-case depressurization conditions (for natural draft appliances)

- $\quad$ spillage testing in worst-case conditions (natural draft heating appliances and water heaters)

- draft testing (natural draft heating appliances and water heaters in worst-case conditions; induced draft heating appliances and water heaters)

- $\quad$ steady-state efficiency measurement in all heating appliances and water heaters

Field technicians recorded oven $\mathrm{CO}$ as an air-free value if possible and recorded oxygen if an air-free value was not available (this feature is instrument-dependent), and recorded both CO and oxygen level for range top burners and unvented heating appliances so as to allow for an air-free calculation.

For natural draft combustion appliances, spillage was evaluated at the draft diverter, draft was measured in the flue, and steady-state efficiency was measured before dilution by ambient air. If there were multiple ports relating to multiple burners a steady-state efficiency test was done in each port. For induced draft appliances spillage testing was not required and all measurements were taken in the flue. For condensing appliances with PVC flues only steady-state efficiency was measured, and this was done at the outlet of the flue on the exterior of the home. Measurements for the steady-state efficiency testing included 
oxygen, $\mathrm{CO}, \mathrm{CO}_{2}$, stack temperature, and efficiency. For oil systems the smoke number was also recorded.

Throughout combustion appliance testing field technicians monitored ambient CO. If the ambient CO level exceeded $35 \mathrm{ppm}$ the field technician turned off the appliance and ceased testing.

The next section of the protocol related to building diagnostics. These included:

- blower door testing

- series leakage zone pressure testing

- pressure pan testing for duct leakage

- blower door subtraction test for duct leakage

- air handler induced pressures

For these tests, outdoor conditions (including a qualitative assessment of wind) were recorded. Step-bystep instructions were provided on the protocol form for each test. Basements were generally considered to be inside for the blower door tests unless they were truly outside the thermal envelope (e.g. they were only accessible from outside). Series leakage tests were done with the zone closed from the house and then with an opening added (e.g. an attic hatch, garage door, etc.) to allow for a quantitative estimation of leakage pathway between the house and that zone. Series leakage tests were done with a priority on zones that had contact to the ground (e.g. crawl spaces) or garages. Attics were a lower priority, and the zoneopened test was not done for attics when opening the hatch would result in insulation being pulled into the home. For air handler induced pressures only one duct zone was assessed, with priority given to foundation zones. Measurements were taken between the house and the zone, the house and outside, and the zone and outside, with three readings taken of each pressure difference to help average out noise due to wind. These readings were taken with the air handler on and then off.

At the conclusion of the data collection form was a checklist reminding field technicians of all necessary steps prior to leaving the home, including required photos.

Field technicians were also trained on conditions that would trigger the issuance of a potentially hazardous condition occupant notification form. In the event of the issuance of this form the field technician indicated the nature and location of the problem and had the homeowner sign two copies, one for themselves and one for the file. Conditions that resulted in issuance of these notification forms included:

- any appliance with a measured CO level above $100 \mathrm{ppm}$

- ambient CO level exceeded 35 ppm

- Identified gas leaks

Field technicians were provided a toll-free call-in number for any field questions, including conditions that were present that warranted discussion regarding how to approach the situation. Any time a potentially hazardous condition form was issued the field technician called in and reported the condition.

Whenever a field technician was going to the field for the first time he or she was accompanied by a field monitor for the first 2-3 homes. This monitor was a member of the project team with detailed experience with the study components. The monitor was there to answer any questions and to provide any additional on-site training required to ensure that the technician was comfortable with the full protocol.

A conference call was held to go over important information for the Phase B round of post weatherization monitor deployment with the technicians. The training covered adjustments to the field protocol to 
include refrigerator monitoring, using the Phase A data sheet, dealing with return visit issue sheet, logging new monitor locations for pick-up technician.

\section{Pick-Up Technicians}

\section{Training Sessions}

Each pick-up technician received a day of training held over two days prior to deployment into the field. The first day of training was held in a classroom and covered an overview of the program, scheduling approach, device recovery, long term equipment operation verification, and the call-in interview. The second day was spent at a site visit to go on a walkthrough of a typical visit, with a return to the classroom for the final logistics around the visits.

There were two trainings with one trainer from APPRISE, the Energy Center of Wisconsin (ECW), and Mathematica Policy Research (MPR) conducting them. The first had 12 participants and the second had 14.

An overview of the Weatherization Program, the Indoor Air Quality Study, the sampling and client selection approach, scheduling and prior visits were reviewed to highlight the importance of the current work. The importance of proper preparation prior to visits was the key to keeping to the schedule and knowing what to expect when arriving at a home. A list of equipment and supplies was provided so technicians were prepared to deal with most problems encountered.

The pick-up technician was given a call-in number if they ran into any difficulties. The pick-up technicians were provided with a training binder that included all of the pertinent forms, the presentation, scripts, client survey and important shipping addresses. The technician also received an Assignment Completion Checklist that was completed and kept on record for each visit. Each technician also was given a second, smaller binder containing only basic instructions and suggested scripts specifically for use in the home. After the first couple days of field work following each training the field techs were debriefed using a conference call to make sure they didn't have any questions.

All Field Techs were required to participate in a one-hour process update on the revised protocol for Phase B data collection before working on those assignments. Field Techs were updated in one of two conference calls. Eleven participated in the first call, while 13 participated in the second call. The calls were hosted by staff from APPRISE, Energy Center of Wisconsin, and Mathematica Policy Research.

\section{Field Protocols}

The goals of each visit were:

First Visit (about one week after the Field Technician visit)

Pick up equipment

- radon canisters

- formaldehyde badges (2 homes per area)

- refrigerator energy loggers, deployed for 1 week (each home as accessible)

- Verify operation of long term equipment

○ passive carbon monoxide loggers, (each home)

$\circ$ temperature/relative humidity loggers, deployed for study duration (each home)

$\circ$ thermocouple loggers (for unvented combustion appliances), (2 homes per area) 
- Inventory secondary refrigerators and freezers

- Facilitate the client making the call-in interview

- Presenting the incentive check

Second Visit (about one week after the field technician's final visit)

Pick up equipment

- radon canisters

- formaldehyde badges (2 homes per area)

- refrigerator energy loggers, deployed for 1 week (each home as accessible)

- passive carbon monoxide loggers, (each home)

- temperature/relative humidity loggers, deployed for study duration (each home)

- thermocouple loggers (for unvented combustion appliances), (2 homes per area)

Present the incentive check

Emphasis was placed on the chain of custody that must be adhered to when picking up the test equipment. Care is needed when sealing the radon and formaldehyde canisters, recording date and time of retrieval and shipping to make sure the paperwork stays aligned with the correct canister. Scripting was used to show how to use the photos and the client to locate installed equipment for retrieval or verification. Completing the documents was part of the field training.

Each of the long term equipment has a visual output that verifies correct operation. Each piece of equipment was demonstrated and details given concerning proper operation. If any problem was discovered, the technician would call in to report the problem and possibly work out a solution. These devices are then recovered during the second visit.

The pick-up technician was required to complete a survey concerning the presence of secondary refrigerators and stand-alone freezers to be completed at the first pick-up visit. Each appliance needed to be described by location and type, whether it is in use and if the space was conditioned.

The technician was to help the client occupant survey while on site. An 800- number was used so there was no cost to the client. The technician was encouraged to be near the client during the interview process, but to not answer the questions for the client. The time was also a good time to verify that all the paper work was complete, all retrievable equipment collected and long term equipment has been identified. 
APPENDIX E. FIELD TECHNICIAN DATA COLLECTION FORM 



\section{APPENDIX E. FIELD TECHNICIAN DATA COLLECTION FORM}

\section{WAP Evaluation IAQ Data Collection Form}

For questions while on site visits, call Tech Hotline 877-297-2797

Keep original of this form, return to Energy Center of Wisconsin per instructions.

Agency Information: $\quad$ Agency ID (4-digit number):

Agency Name:

City:

State: Zip:

Site Information:

Study ID:

Address:

City:

State:

Zip:

Occupant 1 Name:

Phone:

Type:

Best times:

Occupant 2 Name:

Phone:

$$
\text { Type: } \frac{}{\text { (home/cel//work) }} \text { Best times: }
$$

Directions (if house was difficult to find):

(provide directions for subsequent technicians if house was difficult to locate based on address alone.)

\section{All applicable blanks/boxes must be completed}

Take photos of unusual conditions

\begin{tabular}{|c|c|c|}
\hline & Pre-WX: & Post-WX: \\
\hline Agency Tech: & & \\
\hline Date: & (MM/DD/YY) & (MM/DD/YY) \\
\hline Arrival time: & (hh:mm am/pm) & (hh:mm am/pm) \\
\hline
\end{tabular}

Introduction:

Greet owner/occupants, explain project (see talking points)

Explain work to be done, timeline, someone will return next week, then in 1-2 months

Provide Household Info piece

IAQ_DataForm_26.xls

1 of 26 


\section{House - Exterior}

(check all that apply)

$\square$ Single Family Detached $\square$ Single Family Attached

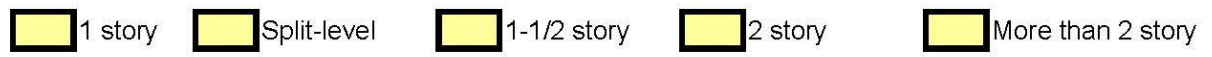

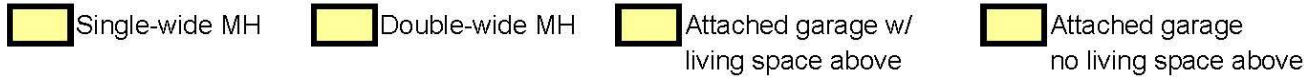

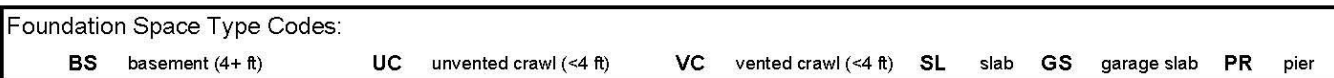

Foundation level

(record all distinct foundation areas, regardless of whether conditioned or unconditioned)

\begin{tabular}{|c|c|c|c|}
\hline $\begin{array}{c}\text { Foot- } \\
\text { print } \\
\text { section }\end{array}$ & $\begin{array}{c}\text { Foundation } \\
\text { space type } \\
\text { (code) }\end{array}$ & $\begin{array}{c}\text { Floor Area } \\
\left(\mathrm{ft}^{2}\right)\end{array}$ & Volume $\left(\mathrm{ft}^{3}\right)$ \\
\hline A & & & \\
\hline B & & & \\
\hline C & & & \\
\hline D & & & \\
\hline E & & & \\
\hline F & & & \\
\hline
\end{tabular}

Take photos from opposite corners to show all sides in 2 or more shots

Sketch Footprint; label sections above; indicate approximate North

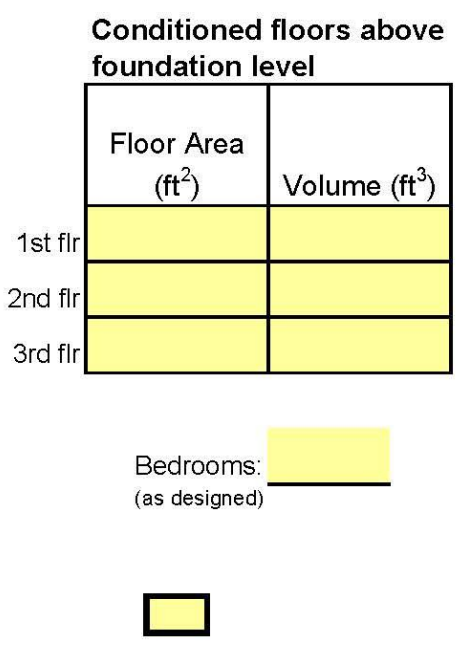

Conditioned floors above foundation level

Bedrooms:

as designed)

Notes: 


\section{Walk Through Home}

Close windows and prep for blower door testing

Identify zones, HVAC systems, exhaust fans, attic access

Observe moisture issues

Identify combustion equipment, especially unvented appliances

Carry combustible gas detector

Note registers: Possible to access? Possible to mask?

\section{Living Space Temperature/RH Logger Installation / Thermostat Settings}

Hang logger from primary thermostat (describe alternate location in Notes)

U10-003 temp/RH logger serial number:

\section{Pre-WX Post-WX}

LED flashes approx every 4 sec:

Photo of installed logger:

Thermostat (as-found settings)

Type (Manual / Programmable):

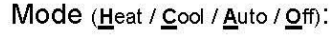

Program status (Program running / $\underline{H}$ old $/)$ : (leave blank if thermostat is not programmable, or mode is Off)

Current temperature setting $(\mathrm{deg} F)$ :

Fan (Auto / 으 / № fan switch):

Closeup photo of thermostat, showing as-found settings:

(open cover if needed to show all switch settings)

Misc Appliance Information

\begin{tabular}{|cll|}
\hline Fuel Codes: & & \\
EL & electric & LP propane \\
NG & natural gas & OT other \\
& & (Describe in Notes)
\end{tabular}

Hot water temperature at kitchen sink (F): (run water until temperature stabilizes; 3 minutes max.)

Number of room or portable A/Cs:

Total number of air handlers:
Kitchen cooktop fuel:

Kitchen oven fuel:

Notes:

$\square$

$\square$

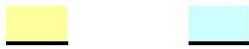

$+\quad-$
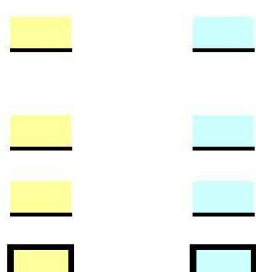

(1)
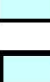
Primary Refrigerator

For return visit, implement this section $O N L Y$ if primary refrigerator has changed since initial visit. Install U12-001 logger in center of food compartment of primary refrigerator for duration of site visit. Install WattsUp? meter on primary refrigerator, if able.

If primary refrigerator is not in the kitchen, record location in Notes.

\begin{tabular}{|llll|}
\hline \multicolumn{2}{l}{ Type Codes: } & & \\
T & top freezer & S & side-by-side \\
B & bottom-freezer & O & other (describe in Notes) \\
\hline
\end{tabular}

Pre-Wx

Post-Wx

(if different than pre- $W x$ )

$\begin{array}{llll}\text { T } & \text { top freezer } & \text { S } & \text { side-by-side } \\ \text { B } & \text { bottom-freezer } & \text { O } & \text { other (describe in Notes) }\end{array}$

Same unit

as Pre-Wx?

Type Code:

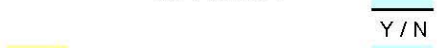

Manufacturer

Model \#:

Year manufactured:

(if listed on unit)

Size:

(food + freezer compartment; if listed on unit)

Rated energy use: (if yellow Energy Guide label present) Defrost Type:
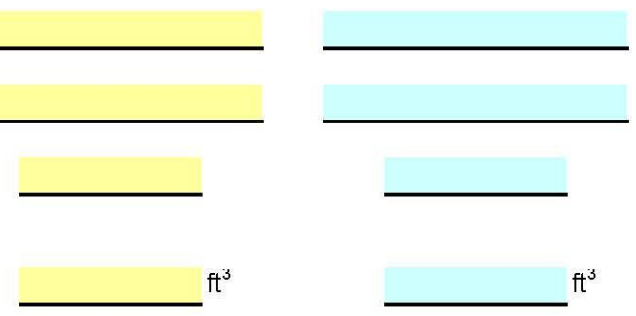

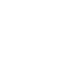

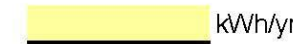

$\mathrm{kWh} / \mathrm{yr}$

kWh/yr

(ㅅuto / Manual / ?) (

Ice maker

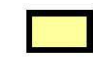

Water dispenser:
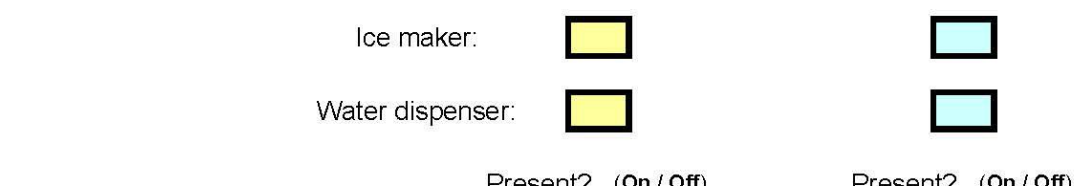

Anti-sweat switch:

Energy Saver (or Power Saver) switch: (Describe setting in notes if not "On" or "Off")

Present? (On / Off)

Present? (On / Off)
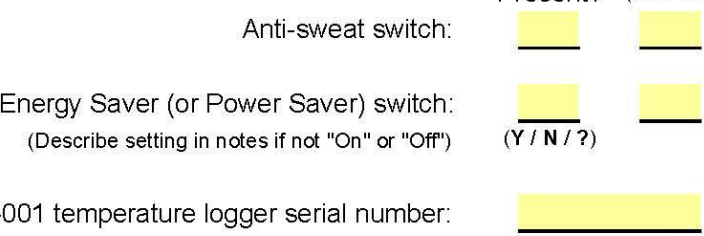

$(\overline{\mathrm{Y} / \mathrm{N} / ?}) \quad(\overline{\mathrm{Y} / \mathrm{N} / ?})$

U12-001 temperature logger serial number: (Install in center of food compart.; verify 4-sec. flashing LED)

WattsUp Meter ID: (3-digit \# on white Energy Center of Wisconsin label) Time WattsUp Meter installed: (to nearest minute)

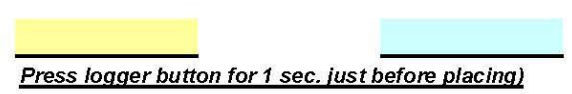

Press logger button for $1 \mathrm{sec}$. just before placing)

\section{Notes:}

Photos of nameplate and unit w/ doors open:

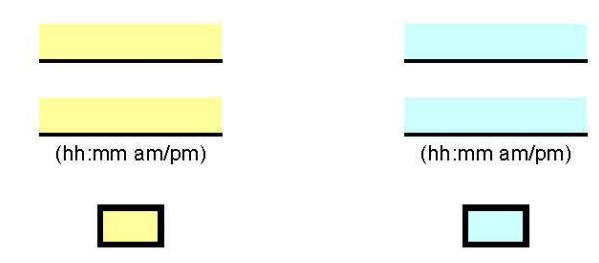

IAQ_DataForm_26.xIs

4 of 26 


\section{Additional Refrigerators}

Install Watts Up meters on up to two additional refrigerators per instructions included in meter shipment Do not install Wattsup meters on refrig in garages, on unheated porches or in other unconditioned spaces. Oniv record unit information below if a meter is deploved on unit. *No U12 loggers are launched for addl refrigerators.

Additional

Additional Type Codes:

Refrigerator

Refrigerator

$\mathbf{T}$ top freezer $\mathbf{S}$ side-by-side

\#1

B bottom-freezer 0 other (describe in Notes)

\#2

Type Code

Manufacturer

Model \#:

Year manufactured

(if listed on unit)

Size

(food + freezer compartment; if listed on unit)

Rated energy use: (if yellow Energy Guide label present) Defrost Type

Ice maker

Water dispenser

Present? (On / Off)

Anti-sweat switch

Energy Saver (or Power Saver) switch: (Describe setting in notes if not "On" or "Off")

Location of additional refrigerator: (e.g. 'basement')

WattsUp Meter ID: (3-digit \# on white Energy Center of Wisconsin label) Time WattsUp Meter installed: (to nearest minute)

Photos of nameplate and unit w/ doors open:
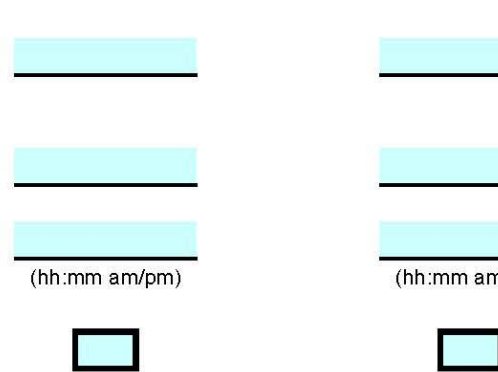

(Áuto / Manual / ?)
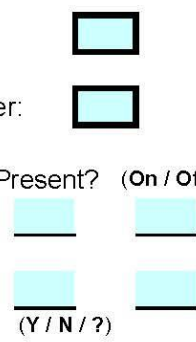

$\mathrm{ft}^{3}$

$\mathrm{kWh} / \mathrm{yr}$

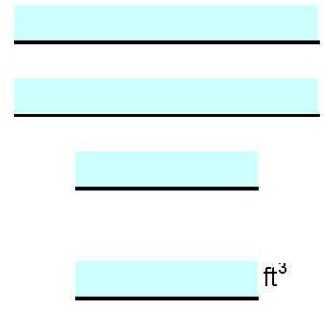

$\mathrm{ft}^{3}$

$\mathrm{kWh} / \mathrm{yr}$

(Auto / Manual / ?)
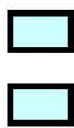

Present? (On / Off)

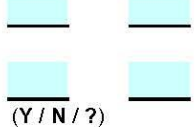

(hh:mm am/pm)

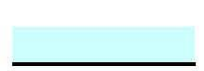

Notes: 


\section{Main living level IAQ sampling}

Place samplers in open living area, away from windows, where unlikely to be disturbed

Always place a radon canister and CO logger

Place formaldehyde sampler based on schedule instructions (2 per 8 homes; 1 st treatment and control)

Describe location in detail (to assist recovery):

(separately for each device if not placed together)

Pre-Wx:

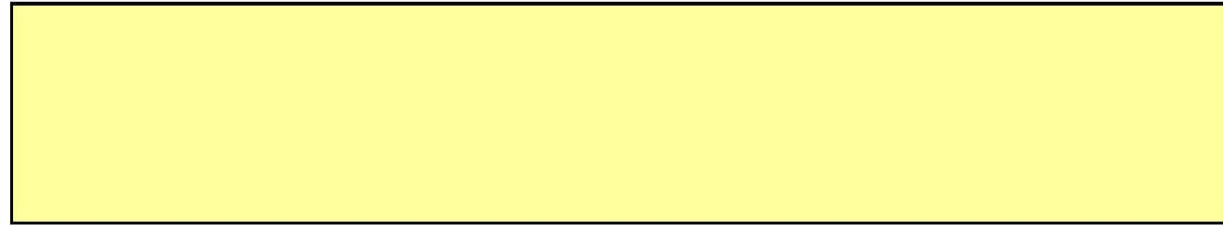

Post-Wx:

Pre-WX

Radon canister number (printed in red on canister) :

Second radon canister also deployed?

Second radon canister number (printed in red):

Formaldehyde sampler ID: (record last 6 chars -2 letters, 4 numbers)

Date/time recorded on all lids and all data sheets:

Photo of placement (from across room):
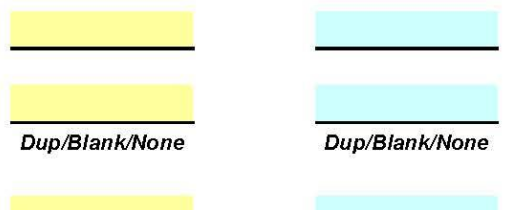

Dup/Blank/None
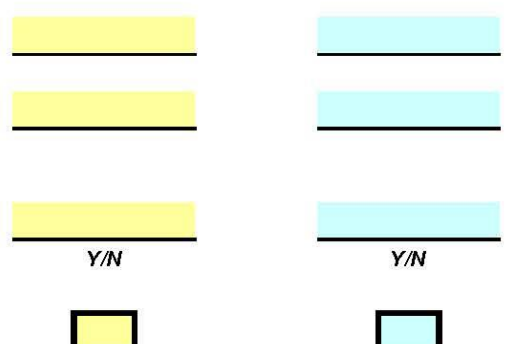

Leave data sheet (folded) and lid inside box, put box under canister for use at pickup

CO logger ID:

Lascar (EL) ID is handwritten CO-xxx, BW is pre-printed serial number (use last 6 digits)

Verify that $\mathrm{CO}$ logger is operational

(BW logger --- shows display; Lascar logger --- LED blinks every 10 seconds) Notes:

IAQ_DataForm_26.xIs 
Above Grade Moisture Observations

Pre-WX

Post-WX

Check here if no signs of moisture

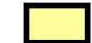

Or...

Record up to six areas with evidence of current or past moisture below.

Describe location sufficiently to allow post-Wx observation.

Make note of decayed drywall, structural wood, etc.

Signs of window condensation include severe paint peeling and rotted sash or sill Photograph all visible evidence of moisture.

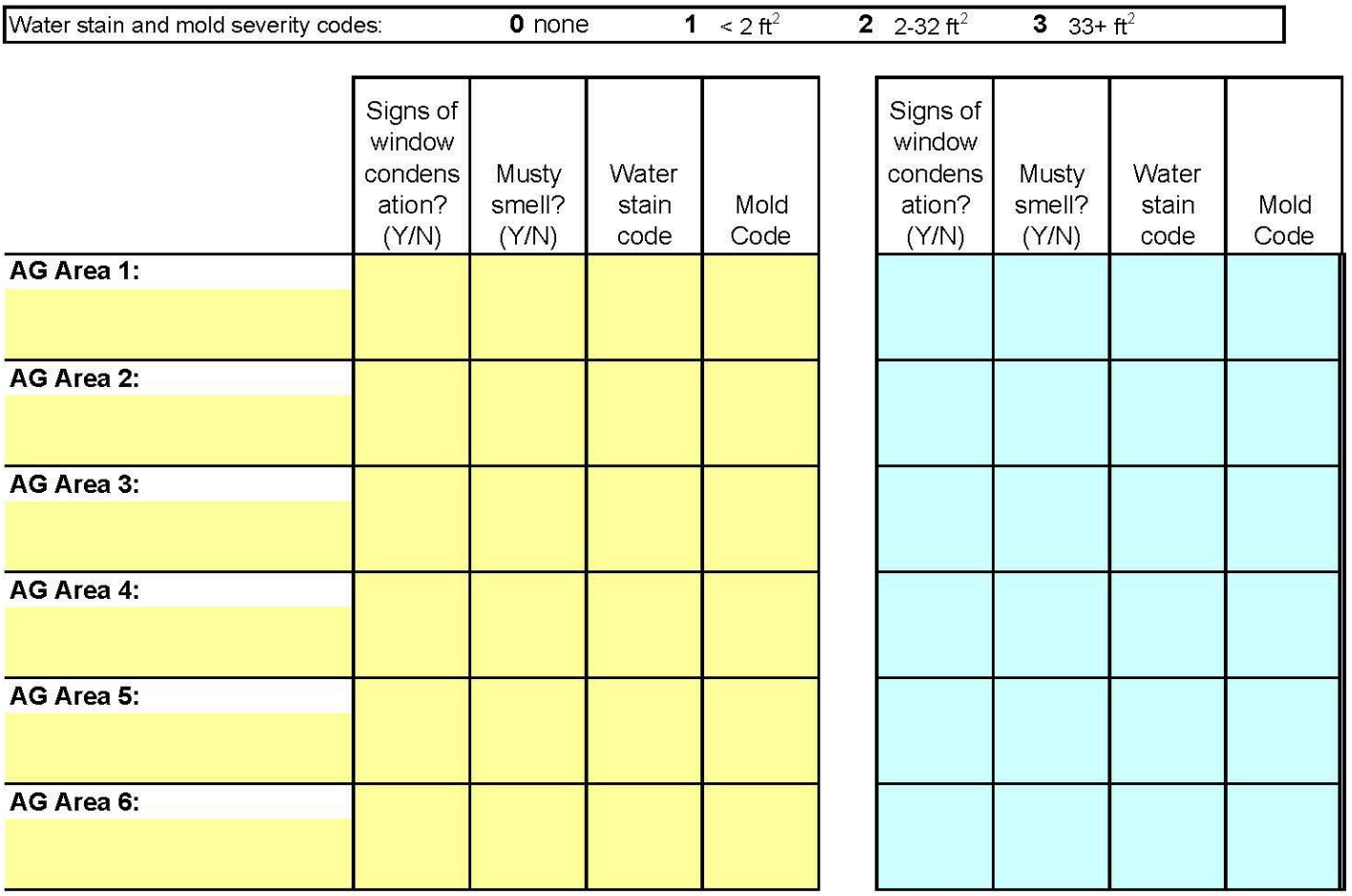

Notes: 


\section{Foundation Details}

Photograph significant cracking, sumps, dirt floors, and visible evidence of moisture.

\begin{tabular}{|c|c|c|c|c|c|c|c|}
\hline Foundation Wall Codes: & $\mathrm{CN}$ & poured concr. & $\mathrm{BL}$ & block & ST & stone/rubble & Other (describe) \\
\hline Foundation Floor Codes: & $\mathrm{PC}$ & poured concr. & DT & dirt & OT & \multicolumn{2}{|c|}{ Other (describe in Notes) } \\
\hline \multirow{2}{*}{\multicolumn{2}{|c|}{ Fdn Space Conditioning Codes: }} & IC & \multicolumn{3}{|c|}{ intentionally conditioned } & \multicolumn{2}{|c|}{ unconditioned } \\
\hline & & UC un & \multicolumn{3}{|c|}{ unintentionally conditioned } & \multicolumn{2}{|c|}{ not applicable (slab) } \\
\hline
\end{tabular}

\begin{tabular}{|c|c|c|c|c|c|c|c|c|}
\hline $\begin{array}{l}\text { Fdn } \\
\text { Section } \\
\text { (from } \\
\text { ftprint) }\end{array}$ & $\begin{array}{c}\text { Fdn Wall } \\
\text { Code }\end{array}$ & $\begin{array}{l}\text { Fdn Wall } \\
\text { height (ft) }\end{array}$ & $\begin{array}{l}\text { Fdn } \\
\text { Floor } \\
\text { Code }\end{array}$ & $\begin{array}{l}\text { Condi- } \\
\text { tioning } \\
\text { Code }\end{array}$ & $\begin{array}{c}\text { Ducts in } \\
\text { space? } \\
(\mathrm{Y} / \mathrm{N})\end{array}$ & $\begin{array}{l}\text { Significant } \\
\text { cracks (below } \\
\text { grade wall / } \\
\text { floor)? } \\
\text { (Y/N/NA) }\end{array}$ & $\begin{array}{c}\text { Percent } \\
\text { exposed dirt } \\
\text { or porous floor }\end{array}$ & $\begin{array}{c}\text { Changes from pre- to } \\
\text { post-WX? (Y/N) } \\
\text { (if } Y \text {, describe on Page } 10 \\
\text { under "Below Grade Sealing } \\
\text { and Ground Cover') }\end{array}$ \\
\hline $\mathrm{A}$ & & & & & & & & \\
\hline$B$ & & & & & & & & \\
\hline $\mathrm{C}$ & & & & & & & & \\
\hline $\mathrm{D}$ & & & & & & & & \\
\hline$E$ & & & & & & & & \\
\hline$F$ & & & & & & & & \\
\hline
\end{tabular}

Foundation Level Moisture Observations

Pre-WX

Post-WX

Check here if no signs of moisture

Or...

Record up to four areas with evidence of current or past moisture below.

Describe location sufficiently to allow post-Wx observation (relate to Areas A-F above). Make note of decayed structural wood

\begin{tabular}{|c|c|c|c|c|c|c|c|c|}
\hline Water stain an & & 0 non & & $<2 \mathrm{ft}^{2}$ & $2 \quad 2-32 \mathrm{ft}^{2}$ & $\begin{array}{l}3 \quad 33 \\
\end{array}$ & & \\
\hline & $\begin{array}{l}\text { Stnding } \\
\text { water } \\
(\mathrm{Y} / \mathrm{N}) \\
\end{array}$ & $\begin{array}{l}\text { Musty } \\
\text { smell } \\
(\mathrm{Y} / \mathrm{N})\end{array}$ & $\begin{array}{l}\text { Water } \\
\text { stain } \\
\text { code }\end{array}$ & $\begin{array}{l}\text { Mold } \\
\text { code }\end{array}$ & $\begin{array}{l}\text { Stnding } \\
\text { water } \\
(\mathrm{Y} / \mathrm{N}) \\
\end{array}$ & $\begin{array}{l}\text { Musty } \\
\text { smell } \\
(\mathrm{Y} / \mathrm{N}) \\
\end{array}$ & $\begin{array}{c}\text { Water } \\
\text { stain } \\
\text { code }\end{array}$ & $\begin{array}{l}\text { Mold } \\
\text { code }\end{array}$ \\
\hline Fdn Area 1: & & & & & & & & \\
\hline Fdn Area 2: & & & & & & & & \\
\hline Fdn Area 3: & & & & & & & & \\
\hline Fdn Area 4: & & & & & & & & \\
\hline
\end{tabular}

Notes:
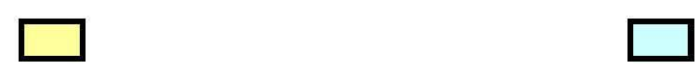


\section{Foundation-Level Radon Canister}

Always place in an accessible basement or crawlspace

Do not deploy undemeath a mobile home on a pier foundation

Describe location in detail (to assist recovery):

Pre-Wx:

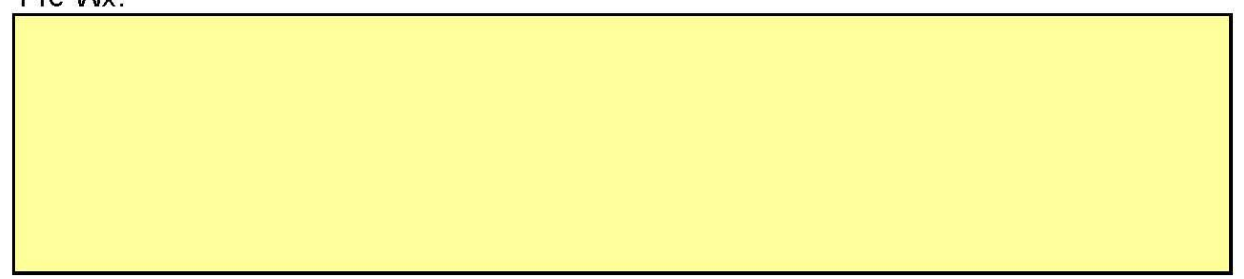

Post-Wx:

Pre-WX

Foundation area $(A, B, C$, etc from table above):

Radon canister number (printed in red on canister) : Second radon canister also deployed?

Second radon canister number (printed in red):

Date/time recorded on lid(s) and data sheet(s):

Photo of placement (from across room):

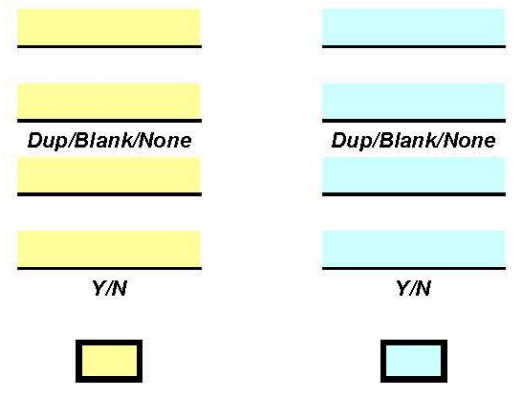

Leave data sheet (folded) and lid inside box, put box under canister for use at pickup

Notes:

IAQ_DataForm_26.xIs

9 of 26

E-11 


\section{Post-Wx Supplementary Data}

\section{Basement}

Is basement occupied for $8+$ hours per week?

(Ask occupants. Record "NA" if no basement present)

Y/N/NA/?

Does basement contain any spaces that are suitable for occupancy ${ }^{*} ?$

(Record "NA" if no basement present)

Y/N/NA

*A space that is suitable for occupancy has:

(1) floor area of $80+$ square feet and average ceiling height of $6+$ feet; and

(2) $50 \%$ or more of wall area finished with a material other than bare or painted concrete or concrete block; and,

(3) at least one heating register or other permanent space heating source or heating outlet

\section{Below Grade Sealing and Ground Cover}

Any signs of recent below-grade sealing or ground cover?

(Refer also to Page 8, Foundation Details)

New covering over dirt floor

New caulk/sealant on below-grade walls/floor

If checked above, describe (and photograph):

Sump:

sealed / unsealed/none

Clothes Dryer (Record "none" below if no clothes dryer present in home)

\begin{tabular}{|cll|}
\hline Fuel Codes: & & \\
EL & electric & LP propane \\
NG & natural gas & OT other \\
& & (Describe in Notes) \\
\hline
\end{tabular}

Clothes dryer fuel code:

Clothes dryer vents to the..

Outside/Inside/CS/Attic

(Note: if gas or LP clothes dryer that is vented inside, measure CO under Unvented Devices)

Notes:

IAQ_DataForm_26.xIs

10 of 26

E-12 
Combustible Gas Leakage

Pre-WX Post-WX

Natural gas or propane used in home?

If no, skip remainder of this page

$\overline{(Y / N)} \quad \overline{(Y / N)}$

If yes, scan basement and gas combustion appliance areas for gas leaks holding end of detector 12" from piping, and complete remainder of this page

\section{Concentrated Leaks}

Concentrated gas leaks are those at which the TPI 775 meter responds with

1 or more RED LED (propane) with detector 12 inches from piping

3 or more RED LED (natural gas) with detector 12 inches from piping

Concentrated gas leaks identified?

$(\overline{N / N / N A)} \quad r / \overline{N / N A)}$

List locations of concentrated gas leaks in Notes

Fill out Notification of Possible Hazards form to identify locations of concentrated leaks

\section{Minor Leaks}

Minor gas leaks are those at which the TPI 775 meter responds at a lower level than indicated above

\[ \text { Minor gas leaks identified? } \]
List locations of minor gas leaks in Notes
Fill out Notification of Possible Hazards form to identify locations of minor leaks

Notes: 


\section{Primary Heating System}

Describe system intended to provide primary home heating.

Pre-WX: Post-WX:

Descriptive name (e.g. gas furnace, oil boiler):

Location:

\begin{tabular}{|llll|}
\hline Location Codes: & & \\
BS & basement & CS & crawlspace \\
AT & attic & GA & garage \\
LS & living space & OT & other \\
& or closet & & (describe in Notes) \\
\hline
\end{tabular}

Fuel:

\begin{tabular}{|llll|}
\hline Fuel Codes: & & \\
NG & natural gas & LP & propane \\
OL & Oil/Kerosene & EL & Electric \\
WD & Wood/pellet & OT & $\begin{array}{l}\text { Other } \\
\text { (describe in Notes) }\end{array}$ \\
\hline
\end{tabular}

Type (select one):

Forced air furnace

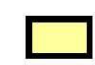

Hot water boiler

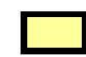

Steam boiler

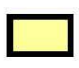

Electric baseboard
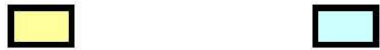

Heat pump (describe type in Notes)

Wood/Pellet stove

Free standing fuel-fired space heater

Fuel-fired wall heater

Other:

\section{Venting (check one)}

Atmospheric w/ draft hood or baro. damper

Induced draft (no draft hood or baro. damper)

Power-vent, sealed-combustion (positive vent pressure; does not use house air) Power-vent, not sealed-combustion (positive vent pressure; uses house air)

Unvented
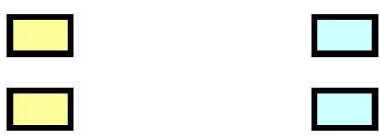

Fuelfired wall heater

$\square$


Primary Heating System (continued)

System functional?

(ask occupants)

Signs of extended flame roll-out?

Potential Issues:
disconnected or leaky venting
downward-sloping venting
leaking boiler
leaking condensate line
fuel-oil leak
no service switch
(see Reference Manual for add'l)

Venting or other issues present?

(see list at left)

(describe in Notes)

Pilot light?

Condensing type?

Humidifier attached?

System also includes central air conditioning?

Estimated \% of annual heating from this system:

(As currently used, if not functional, enter 0 )

If forced air distribution:

$\%$ ductwork distribution:

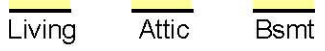

(describe in Notes)

Filter type

\begin{tabular}{|c|c|c|}
\hline \multicolumn{3}{|c|}{ Filter type codes: } \\
\hline ST & standard & PL pleated \\
\hline EL & electronic & OT Other \\
\hline
\end{tabular}

Specifics:

Heating plant $\mathrm{mfr}$ :

Model:

Input rating:

(If not rated, estimate output in notes) Input rating units:

System replaced since start of monitoring?

Take nameplate photo:

Take photo of upstream side of filter:

(If forced air)

Take photo of unit \& surroundings: Add notes on observed venting problems

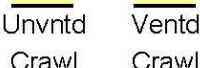

$\overline{\text { Garage }}$

Other
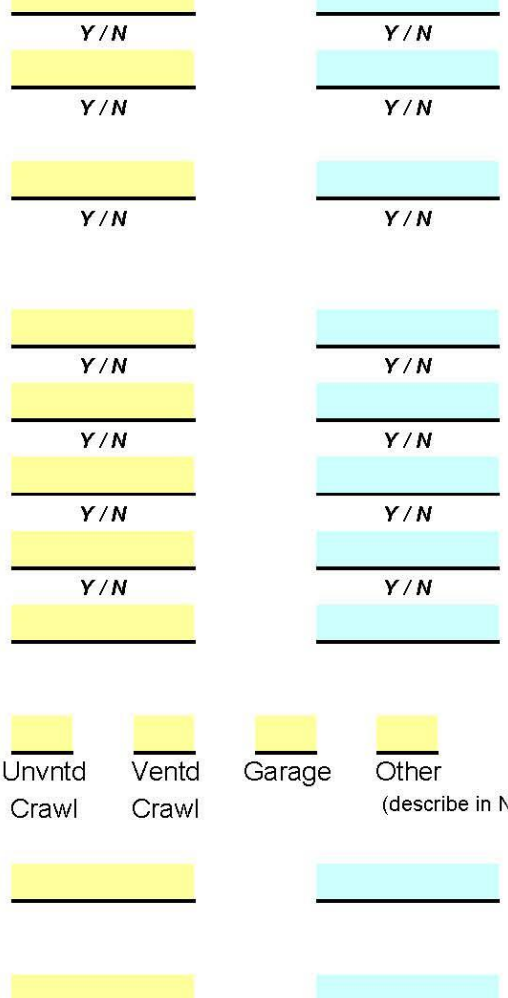

$Y / N / ?$

$Y / N / ?$
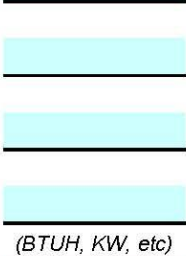

(BTUH, KW, etc)

$\overline{\text { (BTUH, KW, etc) }}$

$Y / N / ?$

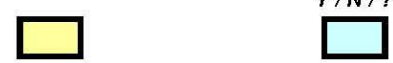

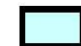

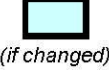

Notes: 


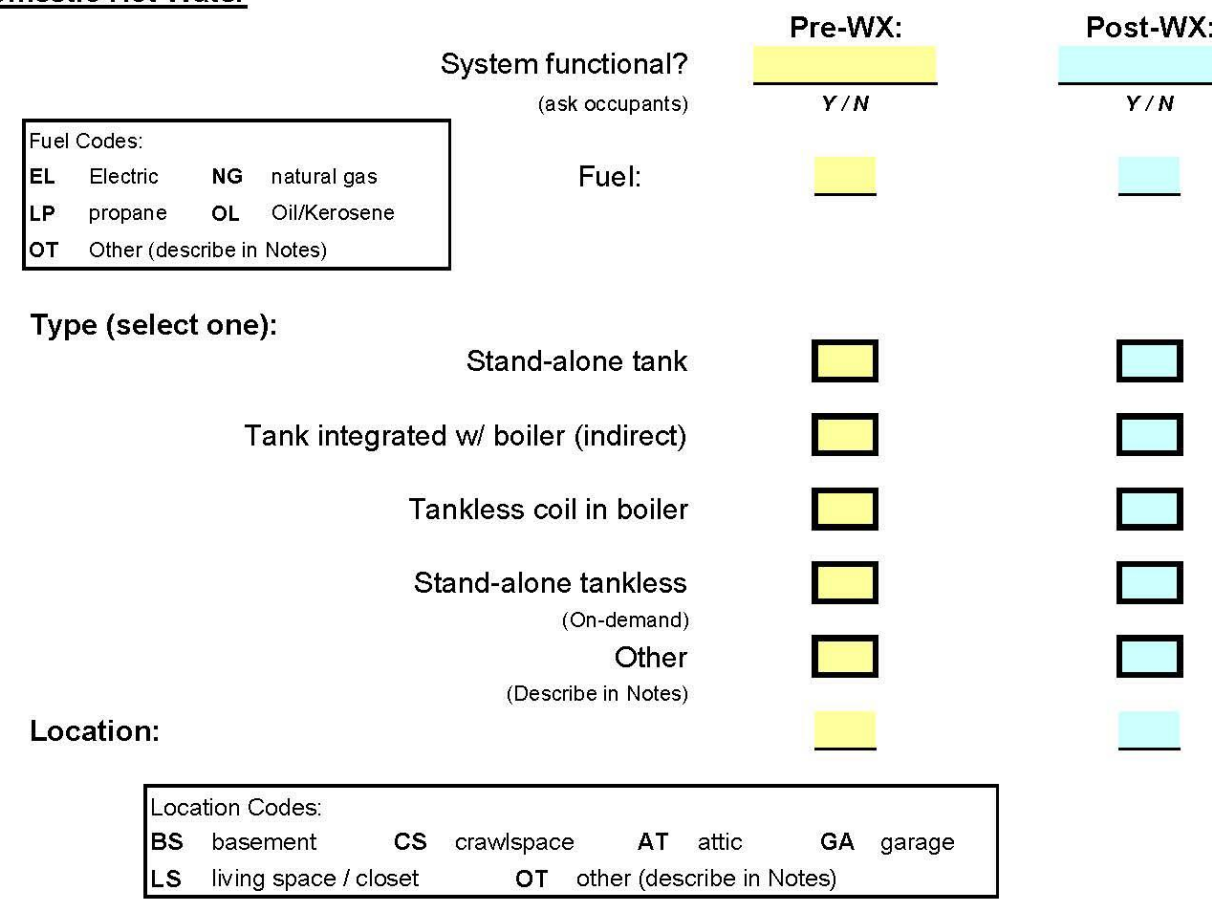

Venting (check all that apply):

Note: describe any venting
system issues such as
disconnected or leaky
connections and
downward-sloping venting
under Notes.

Shared venting with primary space heating

Signs of venting issues?

(see note above)

Other

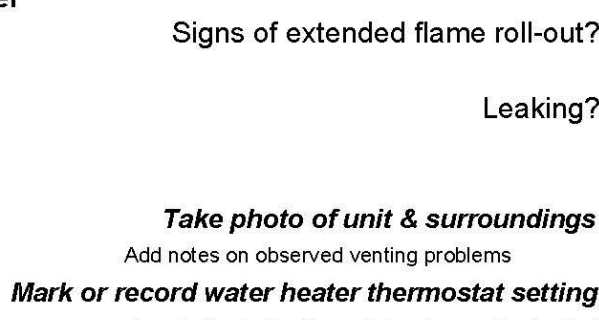

Atmospherically vented

Power vented

Direct (side) vented
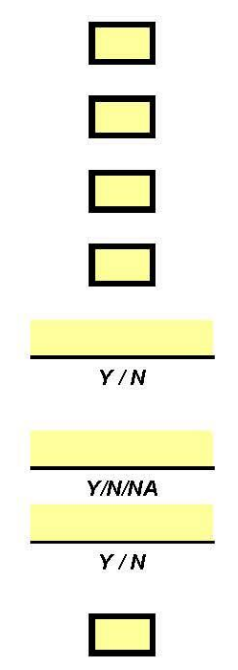

$\square$
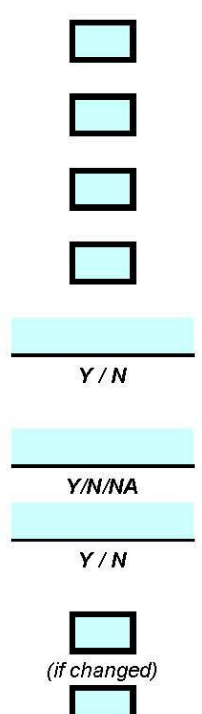

Notes: (needed only if setting will be changed for testing)

IAQ_DataForm_26.xIs 
Water Heater Combustion Testing

Implement test if atmospherically-vented, stand-alone indoor water heater that is functional Allow system to cool for at least 10 minutes after prior operation before performing this test Keep space heating system from operating during this test

Close exterior windows and doors

Close interior doors, except to rooms with exhaust devices

Run hose to measure combustion appliance zone (CAZ) pressure with respect to outside

Set manometer to 10-sec. averaging

CAZ door closed; air handler(s) off; exh. devices off

Pre-WX Post-WX

Record baseline CAZ depressurization:

(measure CAZ with respect to outside)

$\mathrm{Pa}$

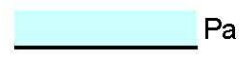

Turn on all exhaust devices, record CAZ depress.:

(kitchen \& bath fans, range hood, dryer, etc, NOT including whole-house fans)

Turn on air-handler, record CAZ depressurization:

Leave air-handler on if increased depressurization

(Repeat for additional air handlers, if present; record values under Notes)

Open CAZ door, record CAZ depressurization:

Leave CAZ door open if increased depressurization.

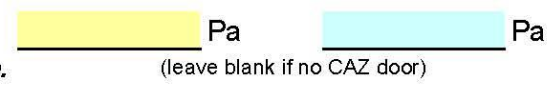

List exhaust devices and final worst-case air-handler and CAZ door status under Notes

Fire water heater, start timer

ABORT test if ambient CO reading exceeds $35 \mathrm{ppm}$ at any point during testing

Peak ambient CO during test (ppm):

Elapsed time to last spillage:

(Record as $>5$ if draft not established w/in $5 \mathrm{~min}$.)

Draft at $5 \mathrm{~min}(\mathrm{~Pa})$ :

(measure downstream of draft hood/diverter; measure with respect to CAZ)
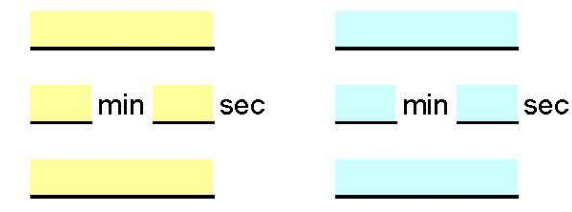

If draft recorded above is stronger than $-4 \mathrm{~Pa}$, record flue $\mathrm{CO}$ and $\mathrm{O} 2$ below.

Otherwise, turn off exhaust fans and air handler, return doors to normal positions, and wait 2 minutes before recording "normal-draft" values below.

Values below are (worst-case/normal-draft):

Flue CO (ppm):

Flue $\mathrm{O} 2(\%)$

Draft $(\mathrm{Pa})$

(measure with respect to $\mathrm{CAZ}$, downstream of draft hood/diverter)

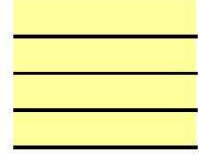

Flue CO exceeding 100 ppm triggers Notification of Possible Hazards form

Disable water heater

If primary heating system to be tested is in same CAZ, maintain worst-case conditions; otherwise, turn off exhaust fans and air handler, and return doors to original positions

Notes:

IAQ_DataForm_26.xIs

15 of 26 
Primary Heating System Worst-Case Draft Test

Implement test if system has draft hood or barometric damper

Allow system to cool for at least 10 minutes after prior operation before performing this test

Close exterior windows and doors

Close interior doors, except to rooms with exhaust devices

Run hose to measure combustion appliance zone (CAZ) pressure with respect to outside

Set manometer to 10 -sec. averaging

If htg. sys in same CAZ as WH, and already in worst-case configuration, check box at right and skip pressure tests below.

Pre-WX Post-WX

\section{CAZ door closed; air handler(s) off; exh. devices off}

Record baseline CAZ depressurization:

(measure CAZ with respect to outside)

$\mathrm{Pa}$

$\mathrm{Pa}$

Turn on all exhaust devices, record CAZ depress.:

(kitchen \& bath fans, range hood, dryer, etc)

Turn on air-handler, record CAZ depressurization:

Leave air-handler on if increased depressurization

(Repeat for additional air handlers, if present; record values under Notes)

Open CAZ door, record CAZ depressurization:

Leave CAZ door open if increased depressurization.

$\mathrm{Pa}$

(leave blank if no $\overline{\mathrm{CAZ} \text { door) }}$

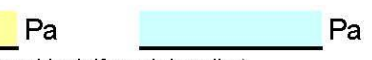

(leave blank if no air handler)

Pa

List exhaust devices and final worst-case air-handler and CAZ door status under Notes

Fire heating system, start timer on burner start

ABORT CAZ testing if ambient CO reading exceeds $35 \mathrm{ppm}$ at any point during testing.

Peak ambient CO during test (ppm):

Time to last spillage (or negative pressure in vent):

(Record as $>5$ if draft not established w/in $5 \mathrm{~min}$.)

Draft at $5 \mathrm{~min}(\mathrm{~Pa})$

(measure downstream of draft hood/diverter; measure with respect to $C A Z$ )

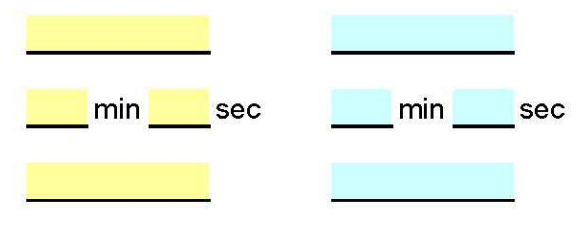

Allow heating system to continue to operate (for SSE combustion and draft test)

Turn off all exhaust devices

Return all doors to normal positions

Proceed to Primary Heating System Steady State Efficiency and Draft Test

Notes:

IAQ_DataForm_26.xIs

16 of 26

E-18 


\section{Primary Heating System Steady State Efficiency and Draft Test}

\section{ABORT CAZ testing if ambient CO reading exceeds $35 \mathrm{ppm}$ at any point during testing}

Omit if condensing system $O R$ has concentric venting (e.g. some mobile home furnaces)

Operate to steady state ( $<5 F$ change in flue temp. over 1 minute)

After steady state reached, (or after $10 \mathrm{~min}$ if not reached) proceed to testing

System appears to be at steady state?

System cycles on high limit?

CAZ ambient air temperature $(F)$ :

$Y / N$

$Y / N$

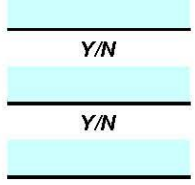

Stack draft $(\mathrm{Pa})$ :

(measure downstream of draft hood/diverter; measure with respect to $C A Z) \overline{\text { (don't need to repeat if measured above) }}$

\section{Smoke \# :}

(Oil systems only) (Heavy smoke may damage combustion analyzer)

If oil, record under \#1 below; if gas or propane record by burner tube number (from left to right)

Pre-WX

Post-WX

\begin{tabular}{|c|c|c|c|c|c|c|c|c|c|c|}
\hline & O2 \% & $\begin{array}{c}\text { co } \\
\text { ppm }\end{array}$ & $\begin{array}{l}\text { Gross } \\
\text { Temp }\end{array}$ & Eff\% & $\mathrm{CO} 2 \%$ & O2 \% & $\begin{array}{c}\mathrm{CO} \\
\mathrm{ppm}\end{array}$ & $\begin{array}{l}\text { Gross } \\
\text { Temp }\end{array}$ & Eff\% & $\mathrm{CO} 2 \%$ \\
\hline $\begin{array}{l}\text { Burner } \\
\# 1\end{array}$ & & & & & & & & & & \\
\hline$\# 2$ & & & & & & & & & & \\
\hline \#3 & & & & & & & & & & \\
\hline$\# 4$ & & & & & & & & & & \\
\hline$\# 5$ & & & & & & & & & & \\
\hline avg & & & & & & & & & & \\
\hline
\end{tabular}

Disable heating system in preparation for blower-door testing.

Seal any venting system holes created for SSE/draft testing.

Avg CO > 100 ppm triggers Notification of Possible Hazards form

Notes: 
Co Testing - Unvented Appliances

Gas or Propane Range/Oven

Pre-WX

Pilot lights present?

$(\mathrm{Y} / \mathrm{N} / ?)$

Post-WX

Range Top

Remove pots from burner area.

Turn all burners to high, and allow to warm up for 3 minutes. Measure 6 inches above flame.

Complete Notification of Possible Hazards form if any burner produces $>50 \mathrm{ppm}$.

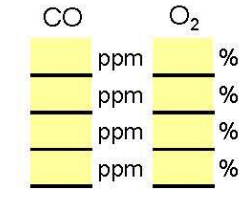

$\mathrm{O}_{2}$

Left rear:

Left front:

Right rear:

Right front:

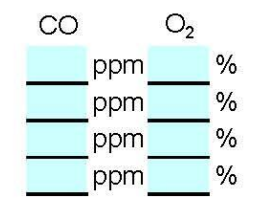

Turn off burners.

Oven

Remove any items stored in oven. Set to Bake at 450-500F.

Test after 5 minutes by inserting probe at center of vent upstream of any dilution air.

Calculate air-free $\mathrm{CO}$. If $>800 \mathrm{ppm}$ air-free $\mathrm{CO}$, wait $5 \mathrm{~min}$. and re-test (record $2 \mathrm{nd}$ rdgs. in Notes)

Complete Notification of Possib/e Hazards form if $>800$ ppm air-free $\mathrm{CO}$ after 2 nd test

Calculation for air-free $\mathrm{CO}$

$\mathrm{CO} \mathrm{ppm}($ air-free $)=20.9 /\left(20.9-\mathrm{O}_{2}\right) * \mathrm{CO} \mathrm{ppm}$

Oven air-free CO:

(From calculation, or air-free ("CO-AF") reading on analyzer)

Turn off oven.

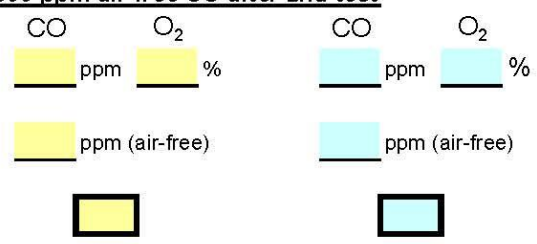

\section{Other Unvented Devices}

List all other unvented combustion devices in home or attached garage.

Operate for 3 minutes before measuring $\mathrm{CO}_{\text {and }} \mathrm{O}_{2}$ (see Reference Manual for locations)

Photograph all listed devices.

Return device controls to original setting after testing.

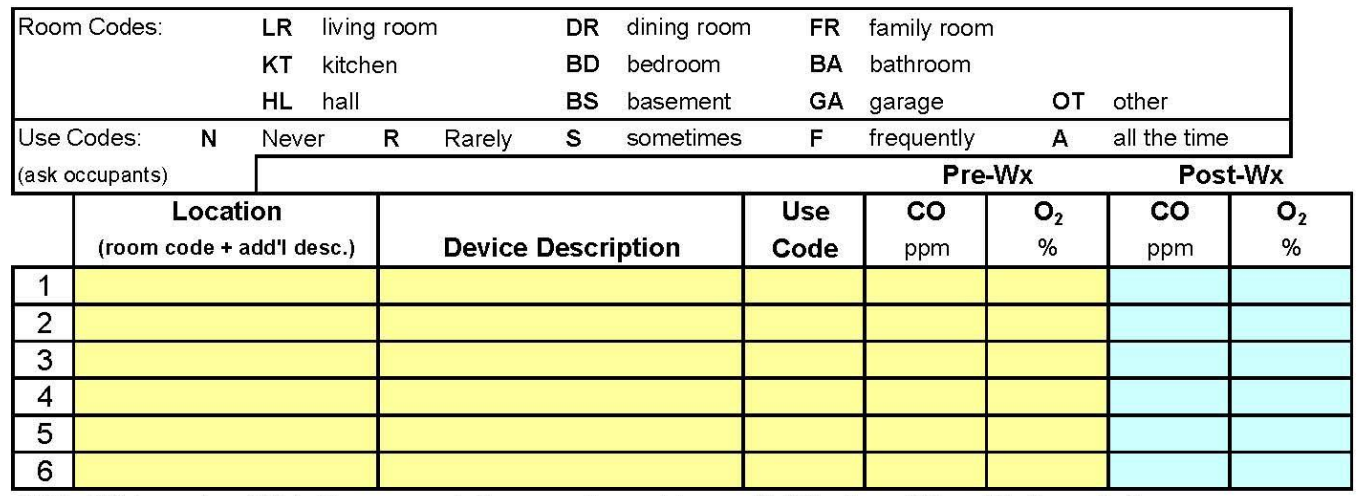

CO $>100$ in vent, or $C O>50$ measured above appliance triggers Notification of Possible Hazards form Notes 


\section{Monitoring of Unvented Appliances}

Install temperature monitoring on unvented combustion appliances, subject to logger availability.

Prioritize as follows:

1. Unvented space heater, tested at $25+p p m \mathrm{CO}$, and reported to be used by occupants

2. Gas or propane oven reported by occupants to be used twice a week or more on average, and tested at 100+ ppm CO (unadjusted, not air-free).

Install U12-014 logger in location where it will be undisturbed, with thermocouple probe mounted to measure a large temperature signal when device operates.

Ensure that logger itself will not be damaged by heat (logger designed for $122 \mathrm{~F}$ )

Push logger button for 3 seconds to activate logging. Verify that LED blinks approx. every 4 seconds.

Photograph device, showing probe location.

\begin{tabular}{|c|c|c|}
\hline $\begin{array}{l}\text { Device } \\
\text { ("Oven" or device number from Unvented Device list) }\end{array}$ & U12-014 logger ID & $\begin{array}{c}\text { LED blinking } \\
\text { every } 4 \text { sec.? } \\
\text { (Y /N) }\end{array}$ \\
\hline & & \\
\hline & & \\
\hline & & \\
\hline & & \\
\hline & & \\
\hline & & \\
\hline
\end{tabular}

Notes: 


\section{Air Leakage, Duct Leakage and Induced Pressures}

Prep for blower door

Outdoor conditions:

Pre-WX

Outdoor CO level (ppm):

Outdoor temperature $(F)$ :

Wind code:
Post-WX

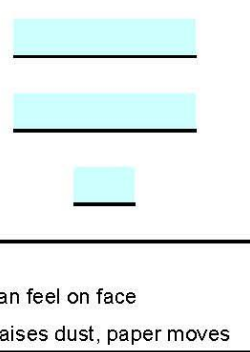

House prep:

First floor air temperature (deg F):

Thermostat(s) turned down or off

$1 \quad 0-3 \mathrm{mph}$; smoke shows direction

2 4-7 mph; can feel on face

3 8-12 mph; leaves, flags move

4 13+ mph; raises dust, paper moves

Digital manometer model used:

Attic hatch closed

Starting position for Interior door to basement (Choose OPEN unless bsmt is clearly outside thermal envelope)
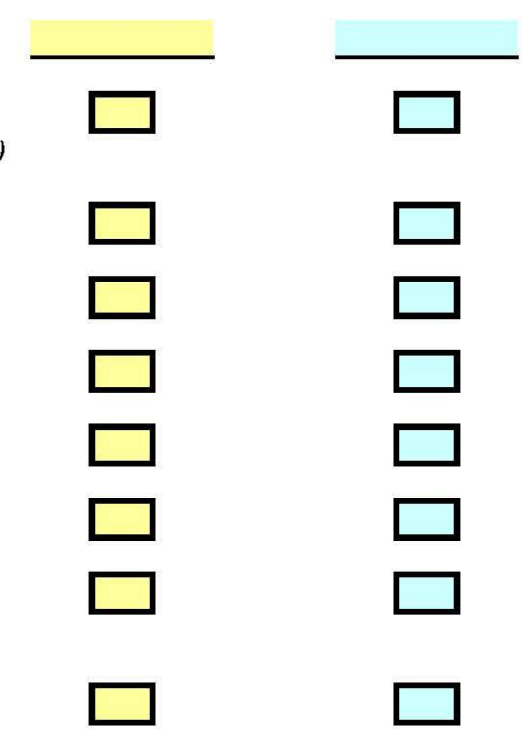

(open/closed/none)

(open/closed/none)

Identify up to 3 zones for zone pressure diagnostics, in the following priority order: attached garages, foundation spaces, attics

Note: follow directions below for basement testing if starting position for basement door is listed as OPEN above. If listed as CLOSED, list basement as Zone 1, and omit basement testing directions below.

IAQ_DataForm_26.xIs 
Leakage and Zone Pressure Diagnostics

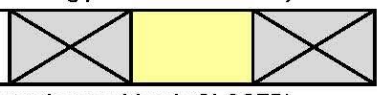

Record ACTUAL pressures and CFM

(unadjusted values)

Record correct sign (negative or positive)

Blower door installed, off \& capped House to outdoors baseline $\mathrm{dP}$

Zone 1 (describe):
Zone 2 (describe):
Zone 3 (describe):
Close door to basement (omit if bsmt door starting position is CLOSED)

Basement

(omit if bsmt door starting position is CLOSED)

Pre-wx

\begin{tabular}{|c|}
\hline \multicolumn{2}{|c}{ Pre-wx } \\
\hline $\mathrm{Pa})$ \\
\hline $\mathrm{atua}$
\end{tabular}
actual actual house - zone outside outside

Open door to basement (omit if bsmt door starting position is CLOSED)
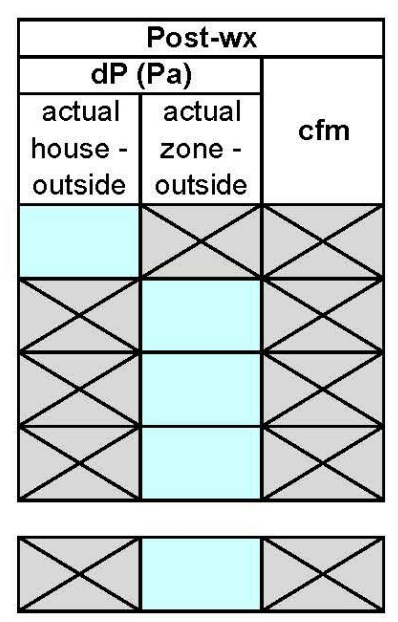

ZONE-CLOSED tests: Uncap Blower door, establish -50 Pa (or highest able, if can't reach 50)

\begin{tabular}{lll} 
House & Zone 1 \\
\hline Zone 2 & Close door to basement, reset to -50 Pa (omit if bsmt door starting position is CLOSED) \\
\hline $\begin{array}{l}\text { Basement } \\
\text { (omit if bsmt door starting position is CLOSED) }\end{array}$ &
\end{tabular}

Open door to basement omit if bsmt door starting position is CLOSED)

ZONE-OPENING tests : Create interior or exterior opening to each zone in turn.

Create interior or exterior opening to Zone 1 , reset to $-50 \mathrm{~Pa}$
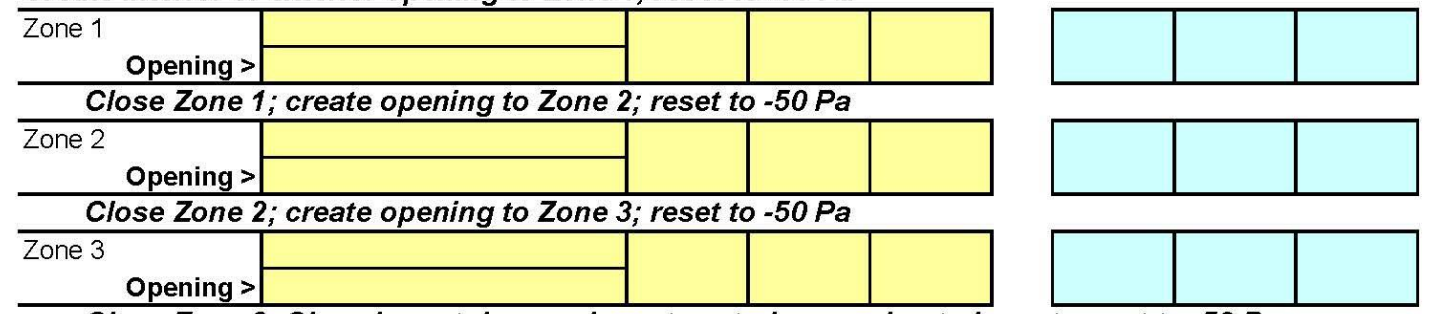

lose Zone 3; Close bsmnt door and create exterior opening to bsmnt; reset to $-50 \mathrm{~Pa}$

(omit if bsmt door starting position is CLOSED or unable to create exterior opening)

Basement (omit if bsmt door starting position is CLOSED

or unable to create exterior opening to bsmnt)

Close exterior opening to basement

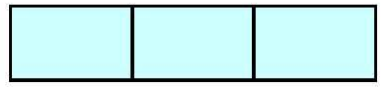


Air handler induced pressures

Conduct test if forced-air ductwork is present.

Measure pressures in one space containing ductwork, in the following priority order:

(1) Foundation space with dirt floor

(2) Unvented crawispace

(3) Basement - Close basement door for testing (omit if no basement door)

(4) Vented craw/space

(5) Attic (measure attic pressures only if hose already in place from zone testing)

Describe space selected for measurement:

(relate to Areas A-F if foundation space)

Which HVAC system (primary or secondary)?

Blower door off and capped.

Manometer set for 10-second averaging.

Record consecutive 10 -second readings of each pressure channel.

Pre-WX

$\mathrm{dP}(\mathrm{Pa})$

\begin{tabular}{|c|c|c|}
\hline actual & actual & actual \\
house - & zone - & house - \\
outside & outside & zone \\
\hline
\end{tabular}

Turn air handler on, wait 30 sec. for ramp-up.

\begin{tabular}{|l|l|l|l|}
\hline Test $1 \mathrm{a}$ & & & \\
\hline Test $1 \mathrm{~b}$ & & & \\
\hline Test $1 \mathrm{c}$ & & & \\
\hline
\end{tabular}

Turn air handler off, wait 30 sec. for ramp-down.

\begin{tabular}{|l|l|l|l|}
\hline Test $2 a$ & & & \\
\hline Test $2 b$ & & & \\
\hline Test $2 c$ & & & \\
\hline
\end{tabular}

Return air handler to normal operating mode.
Post-WX

\begin{tabular}{|c|c|c|} 
dP (Pa) \\
\begin{tabular}{|c|c|c|}
\hline actual & actual & actual \\
house - & zone - & house - \\
outside & outside & zone \\
\hline
\end{tabular}
\end{tabular}
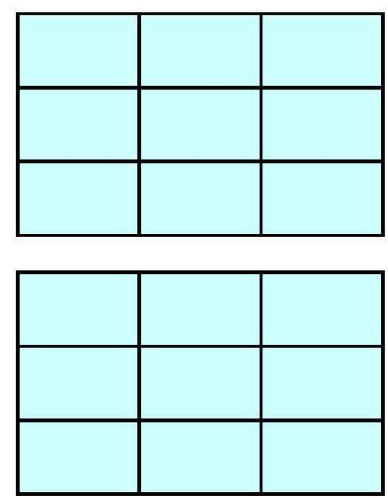


\section{Pressure Pan Duct Leakage Testing}

Open basement door for testing

Set blower door to maintain $-50 \mathrm{~Pa}$

Work clockwise around home and within each room starting at entry

Measure pressure-pan pressures with respect to house

Record inaccessible registers on form, with "NA" for pressure reading

Level: $0=$ below-grade, 1 and higher above-grade

\begin{tabular}{|llll|}
\hline Room Codes: & LR living room & BA & bathroom \\
& DR dining room & HL hall \\
& FR family room & BS basement \\
& KT kitchen & GA garage \\
& BD\# bedroom num \# & OT other \\
\hline
\end{tabular}

\begin{tabular}{|lll|}
\hline Register types: & & \\
WL wall (low) & TK toe-kick \\
WH wall (high) & CL ceiling \\
FL floor & & \\
BS baseboard & & \\
\hline
\end{tabular}

Level

$(0,1,2) \quad$ Room (code + add'l descr.)

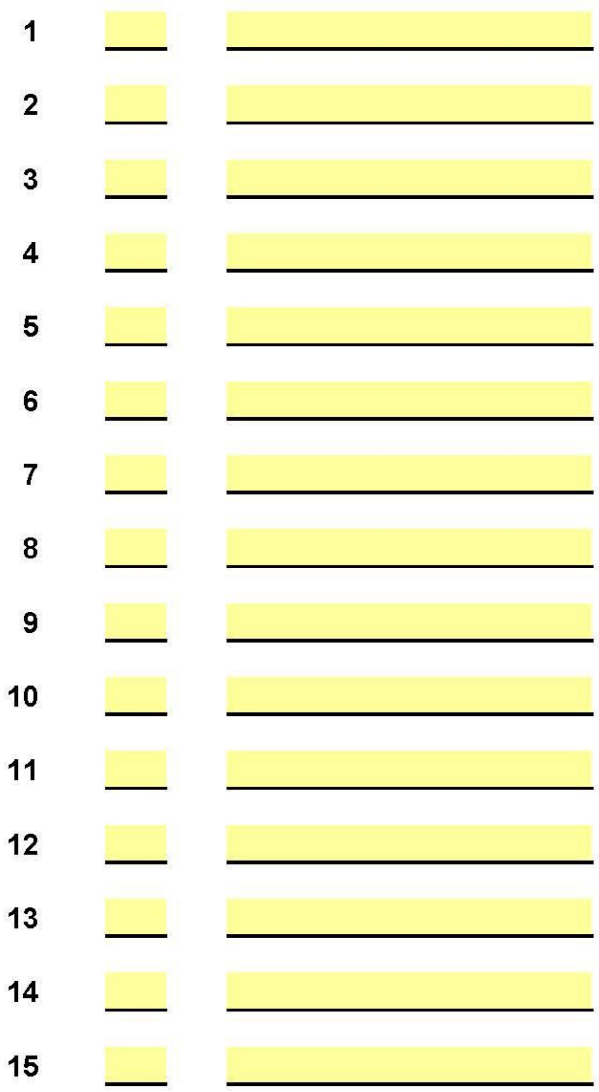

(Use supplemental sheet if needed)
Supply or Register

Return Type
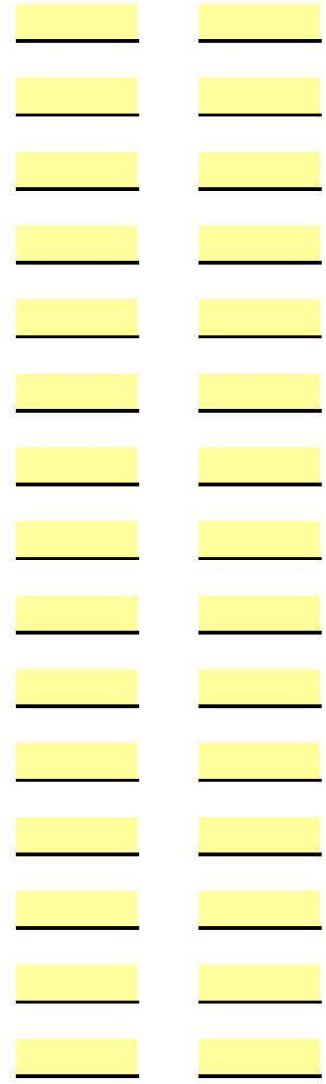

Pressure Pan dP

(wrt house)

Pre-Wx Post-Wx

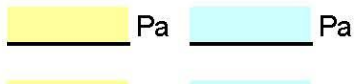

$\mathrm{Pa}$ $\mathrm{Pa}$

$\mathrm{Pa}$ $\mathrm{Pa}$
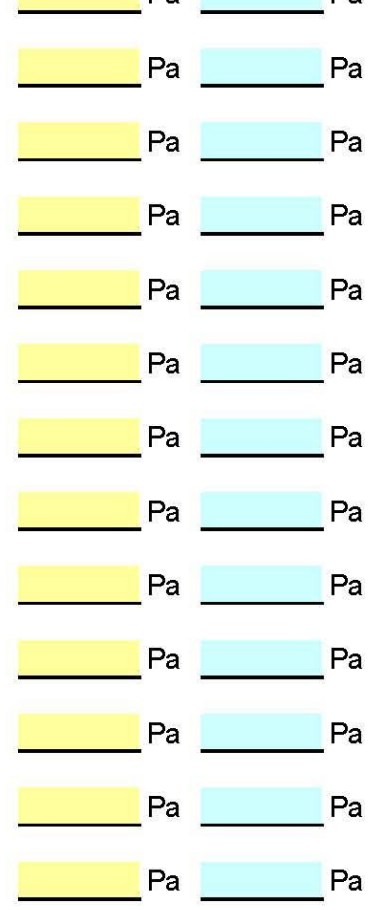

$\mathrm{Pa}$

$\mathrm{Pa}$

23 of 26 


\section{BD Subtraction Duct Leakage Test}

Perform if any of the following are true:

a) Air handler is outside the pressure envelope (e.g. attic, garage, or crawl space)

b) $50 \%$ or more of a duct system is outside the pressure envelope

c) A single pressure-pan reading exceeds $8 \mathrm{~Pa}$, or 4 readings exceed $4 \mathrm{~Pa}$

Select the zone outside the pressure envelope that has the largest amount of ducting in it; use this zone for zone measurements below.

Open basement door for testing (unless basement is clearly outside thermal envelope, as decided previousiy for blower door testing).

Place duct pressure tube inside duct system at a register with low pressure pan reading

Describe zone selected for measurement:

Which HVAC system (primary or secondary)?

\section{Pre-Wx}

\begin{tabular}{|c|c|c|c|}
\hline $\begin{array}{c}\text { actual } \\
\text { house - } \\
\text { outside }\end{array}$ & $\begin{array}{c}\text { actual } \\
\text { zone - } \\
\text { outside }\end{array}$ & $\begin{array}{c}\text { actual } \\
\text { duct - } \\
\text { inside }\end{array}$ & cfm \\
\hline
\end{tabular}

Post-Wx

\begin{tabular}{|c|c|c|c|}
\hline actual & actual & actual & \\
house - & zone - & duct - & cfm \\
\hline
\end{tabular}

Mask (seal) all registers.

Mask (seal) filter slot if air handler is in intentionally conditioned space.

Blower door off and capped.

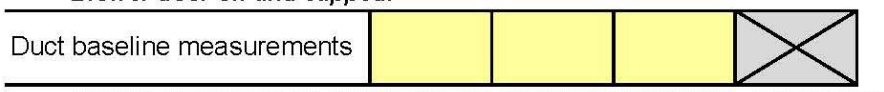

Uncap Blower door, establish -50 Pa (or highest able, if can't reach 50)

Duct pressure test

Remove all masking

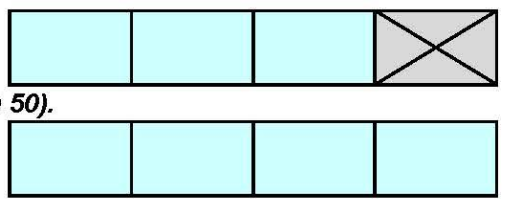


Final House-to-Outside Baseline Pressure

Conduct test just before breaking down blower door

Blower door off and capped.

Measure house-outside baseline pressure (minimum 10-second average)

Be sure to record correct sign.

\begin{tabular}{|c|c|c|}
\hline \multicolumn{3}{|c|}{ Pre-wx } \\
\hline$\overline{\mathrm{dP}}$ & Pa) & \\
\hline $\begin{array}{c}\text { actual } \\
\text { house - } \\
\text { outside }\end{array}$ & $\begin{array}{l}\text { actual } \\
\text { zone - } \\
\text { outside }\end{array}$ & $\mathrm{cfm}$ \\
\hline
\end{tabular}

\begin{tabular}{|c|c|c|}
\hline \multicolumn{3}{|c|}{ Post-wx } \\
\hline \multicolumn{2}{|c|}{ dP (Pa) } & \multirow{2}{*}{ cfm } \\
\cline { 1 - 2 } $\begin{array}{c}\text { actual } \\
\text { house - }\end{array}$ & $\begin{array}{c}\text { actual } \\
\text { house - } \\
\text { outside }\end{array}$ & \\
zone & \\
\hline
\end{tabular}

House to outdoors baseline $\mathrm{dP}$
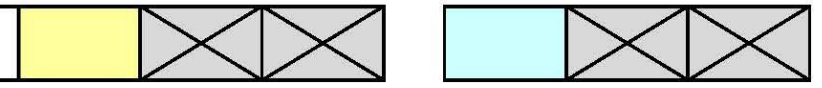

Notes (record all blower door, zone pressure testing and duct leakage notes here): 
Final Check List

Thermostat, all switches, pilots, returned to normal operating status

Combustion appliances checked and are functioning as found

Refrigerator temperature logger retrieved

(press logger button for 1 sec. iust after removing)

Photo of CO logger as placed (room view)

Photo(s) of radon canister(s) as placed (room view)

Photo of formaldehyde sampler as placed

Photos of other combustion devices

Photo of thermocouple logger(s) if installed (room view)

Photo of Temp/RH logger as installed (room view)

Photos of furnace/boiler and nameplate (in focus)

Photo of water heater

Photos of house exterior (opposite corners)

Present and discuss Household Info (1-page info piece)

Thank You to occupants
Pre-wX

Post-WX

$\square^{\mathrm{YN}}$

$\square$ Y

$\square_{\mathrm{Y} / \mathrm{N}} \quad \square_{\mathrm{YIN}}$

$\square^{\mathrm{YN}} \quad \square^{\mathrm{YN}}$

$\square^{\mathrm{YN}} \quad \square_{\mathrm{YN}}^{\mathrm{N}}$

$\square \mathrm{YN}$

$\square^{Y / N}$

$\square$ Y

$\square$ YN

$\square_{\mathrm{YN}} \quad \square_{\mathrm{YN}}$

$\square$ Y IN

$\square_{Y / N}$

$\square_{\mathrm{Y} / \mathrm{N}} \quad \square_{\mathrm{YN}}$

$\square_{\mathrm{YN}}^{\mathrm{YN}} \square_{\mathrm{YN}}$

$\square \mathrm{YN}$

$\square$ Y

$\square^{\mathrm{Y} N} \quad \square^{\mathrm{Y} / \mathrm{N}}$

$\square$ YIN

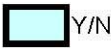

$\square \mathrm{YNN}$

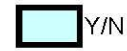

If any potential carbon monoxide or combutible gas hazards were identified, complete Notification of Possible Hazards form

Notification of Possible Hazards form was prepared: (prepare duplicate copies for occupants and project team)

Pre-WX: Call Tech Hotline in each case when this form is used.

Obtained adult signature on form:

Presented check or gift card to occupant:

$Y / N / N A$

$Y / N$

Obtained adult signature for check or gift card:

Leave time:

$\frac{Y / N / N A}{(A M / P M)}$

Post-WX:

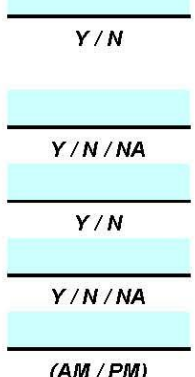

Important: complete and upload an electronic version of this form within 24 hours. Notes:

IAQ_DataForm_26.xIs

26 of 26 


\section{APPENDIX F. PICK-UP TECHNICIAN DATA COLLECTION FORM}





\section{APPENDIX F. PICK-UP TECHNICIAN DATA COLLECTION FORM}

\section{WAP Evaluation IAQ Sampler Pick-Up Form}

For questions while on site visits, call Tech Hotline 877-297-2797

Return to APPRISE per instructions.

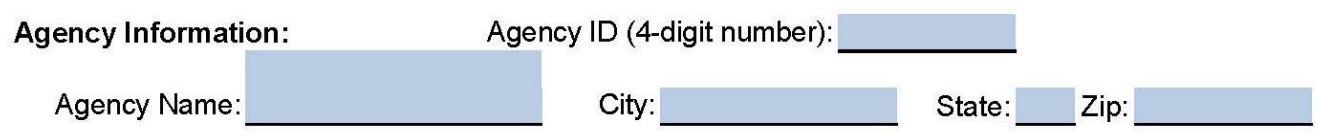

Site Information:

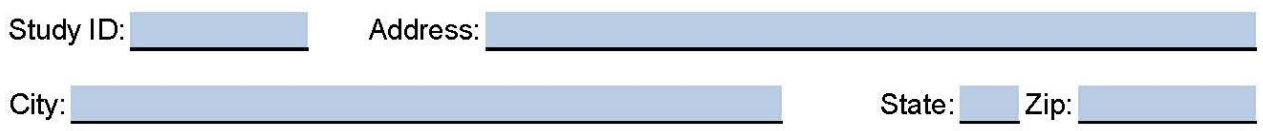

City:

Zip:

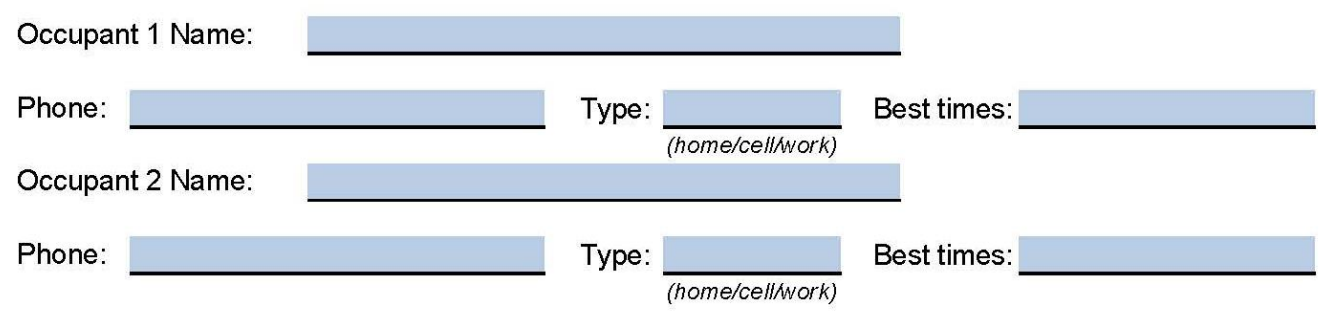

All applicable blanks/boxes must be completed

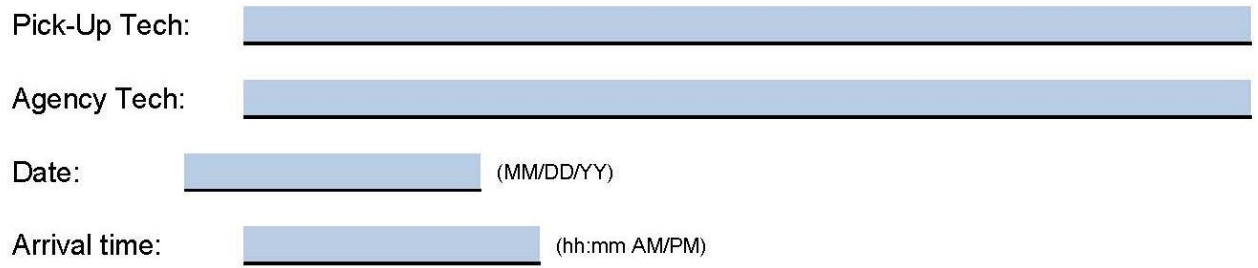

Special instructions for this site:

Directions to this site: 


\section{IAQ Samplers}

Retrieve radon and formaldehyde samplers

Location for Foundation Level Samplers

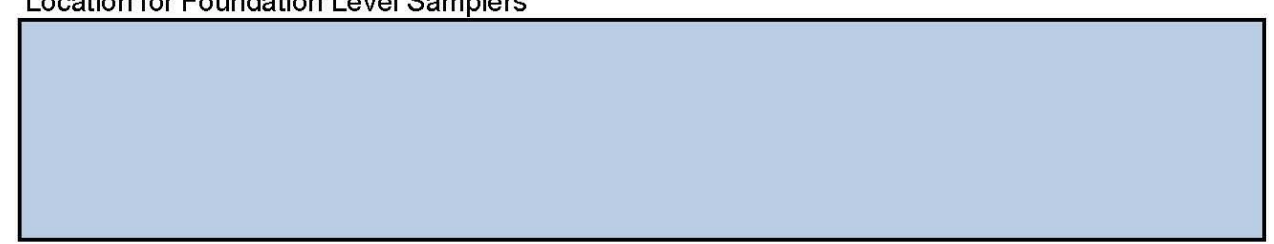

Location for Main Living Space Samplers

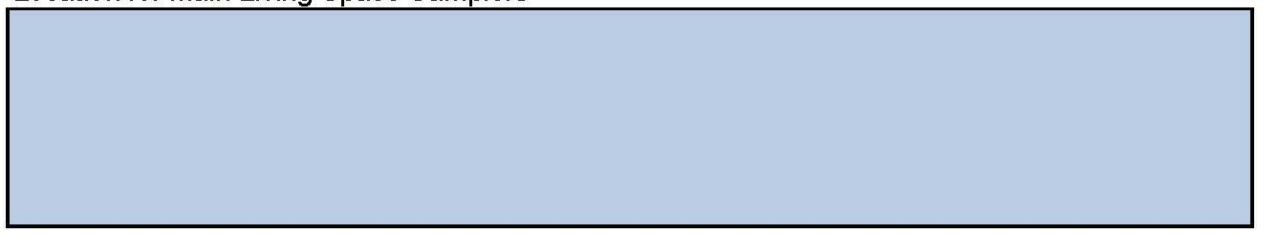

Radon

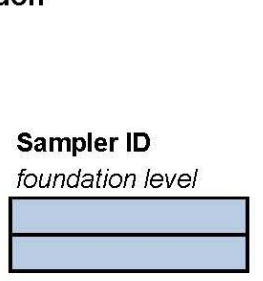

\section{As-Found}

open

s ealed

Not found

$\stackrel{ }{\square}$

main living level
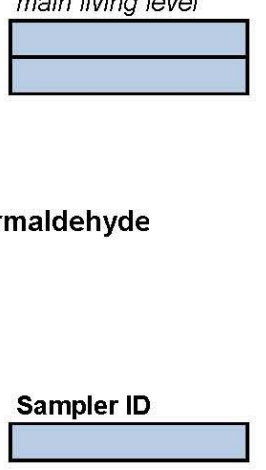

Notes:

\section{Notes:}

IAQ_pickup_form_06.xls 


\section{Primary Refrigerator Logger Retrieval}

Retrieve meter on primary refrigerator. (OK to leave extension cord behind.)

(If not comfortable moving refrigerator to retrieve meter, leave in place, and select " $U$ " below.)

$\begin{array}{ll}\text { Found } & \text { Not found } \\ \text { Primary Refrigerator } & \text { Unable to retrieve } \\ \text { WattsUp Meter ID }\end{array}$

Retum refrigerator to normal position, if moved to retrieve meter.

\section{Secondary Refrigerator/Freezer Inventory}

Record all secondary refrigerators and stand-alone freezers in the home.

Do not include the primary refrigerator.

\begin{tabular}{|c|c|c|c|c|c|}
\hline & Room code & Type code & $\begin{array}{l}\text { Plugged in? } \\
(\mathrm{Y} / \mathrm{N})\end{array}$ & $\begin{array}{l}\text { Is unit in an } \\
\text { intentionally-co } \\
\text { space? }(\mathrm{Y} / \mathrm{N} / \text { ? }\end{array}$ & \multirow{7}{*}{$\begin{array}{l}\text { (note: "intentionally-conditioned" means } \\
\text { the unit is in a room that is heated } \\
\text { or cooled similarly to the main living area } \\
\text { of the home.) }\end{array}$} \\
\hline 1 & & & & & \\
\hline 2 & & & & & \\
\hline 3 & & & & & \\
\hline 4 & & & & & \\
\hline 5 & & & & & \\
\hline 6 & & & & & \\
\hline
\end{tabular}

\begin{tabular}{|rl|}
\hline Room codes: & \\
BS & basement \\
GA & garage \\
PR & porch \\
KT & kitchen or pantry \\
HL & hall or mudroom \\
LS & other living-space location \\
OT & other location not in living space* \\
*describe in Notes & \\
\hline
\end{tabular}

\begin{tabular}{|l|l|}
\hline Type codes for refrigerators: \\
T & Top-freezer \\
B & Bottom-freezer \\
S & Side-by-side \\
G & Single-door \\
C & Compact \\
O & Other (describe in Notes \\
\hline & \\
\hline Type codes for freezers: \\
U & Upright freezer \\
H & Chest freezer \\
\hline
\end{tabular}

Notes: 


\section{Data Logger Check}

Check that installed data loggers are in place and functioning properly.

If a logger appears NOT to be logging, call Tech Support (877-297-2797) for further direction.

Do not remove these loggers from the home, unless instructed otherwise by Tech Support.

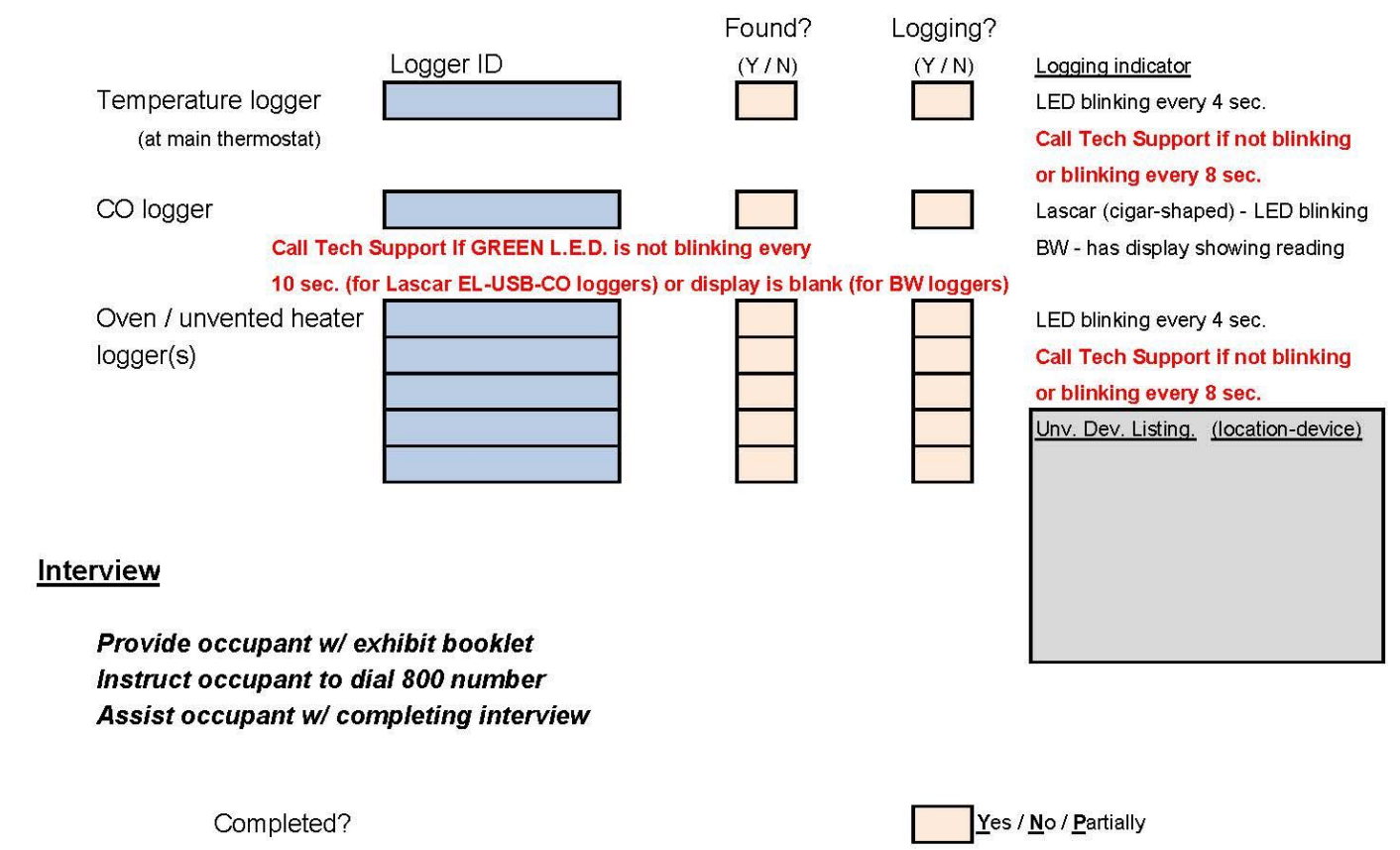

Incentive

Check or gift card provided?

$\square \underline{Y}$ es / №

Final Checklist
Radon / formaldehyde samplers removed from home?
yes / №
Refrigerator logger removed from home?
$\square$ Yes / №
Primary refrigerator in normal position?
yes / №

Notes: 


\section{WAP Evaluation IAQ Sampler Pick-Up Form - Phase B}

For questions while on site visits, call Tech Hotline 877-297-2797

\section{Return to APPRISE per instructions.}

Color code for form completion: Pre-filled (Grey), Pick-Up Tech (Pink), Install Tech (Blue)

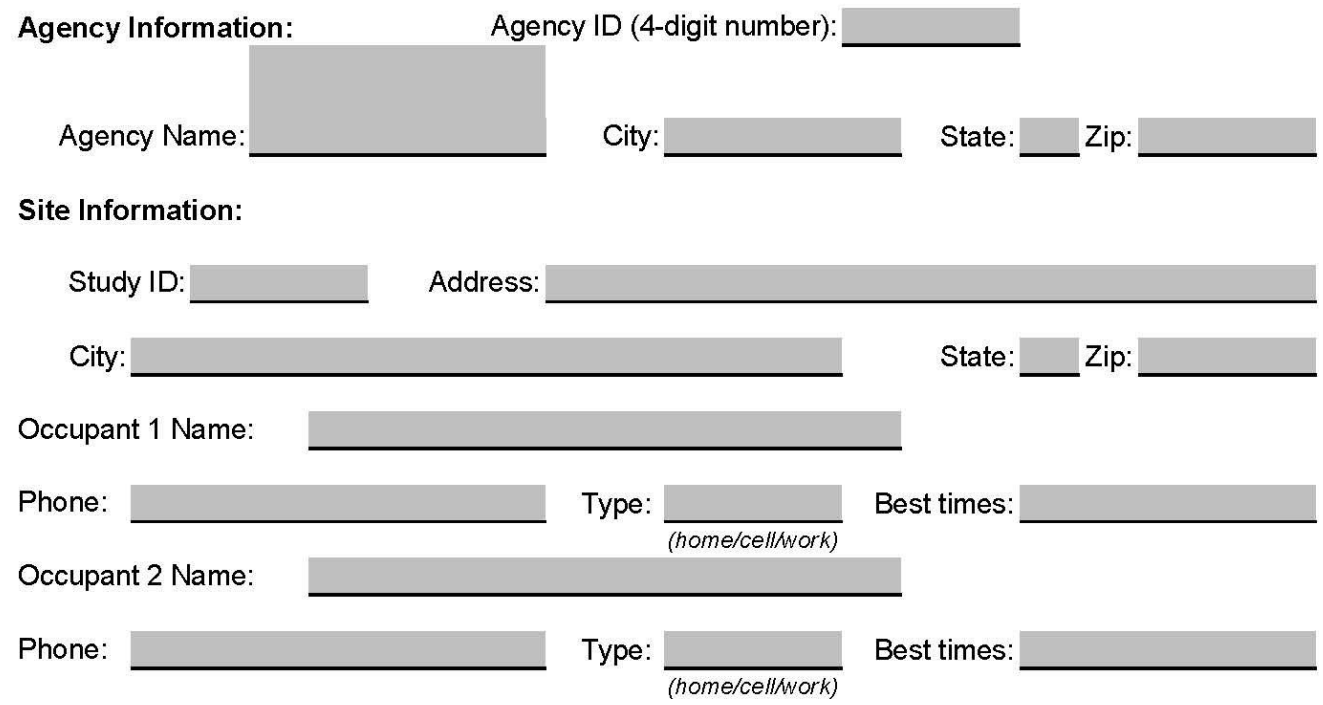

All applicable blanks/boxes must be completed

Pick-Up Tech:

Agency Tech:

Date:

(MM/DD $Y Y$ )

Arrival time:

(hh:mm AM/PM)

|Special instructions for this site:

Directions to this site: 


\section{IAQ Samplers}

Retrieve radon and formaldehyde samplers

Call tech support if you are unable to locate and retrieve any samplers Location for Foundation Level Samplers

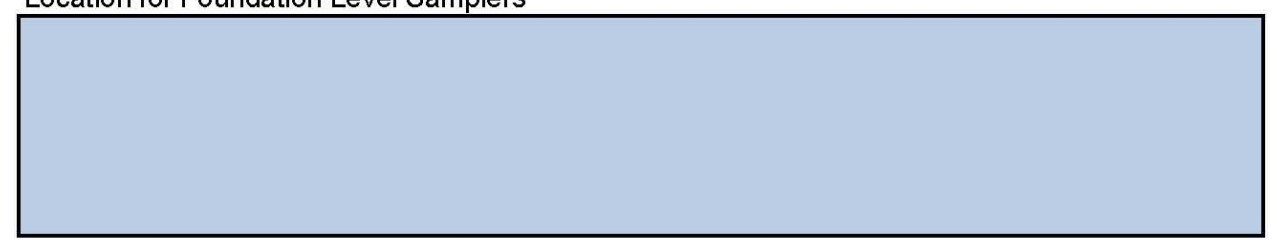

Location for Main Living Space Samplers

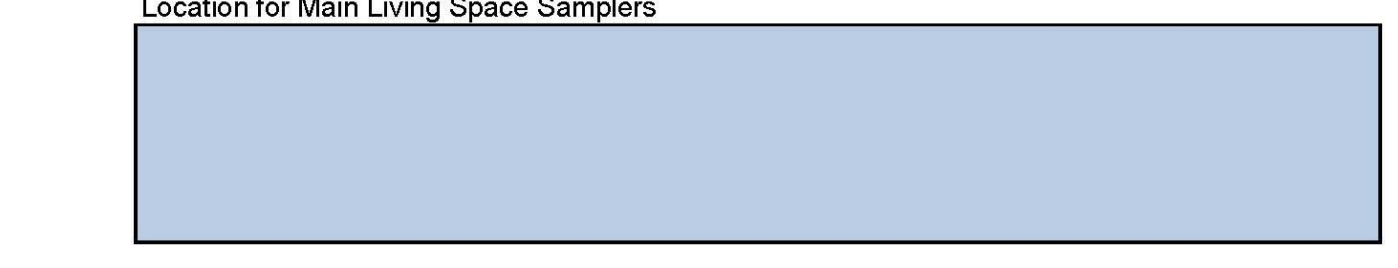

Radon
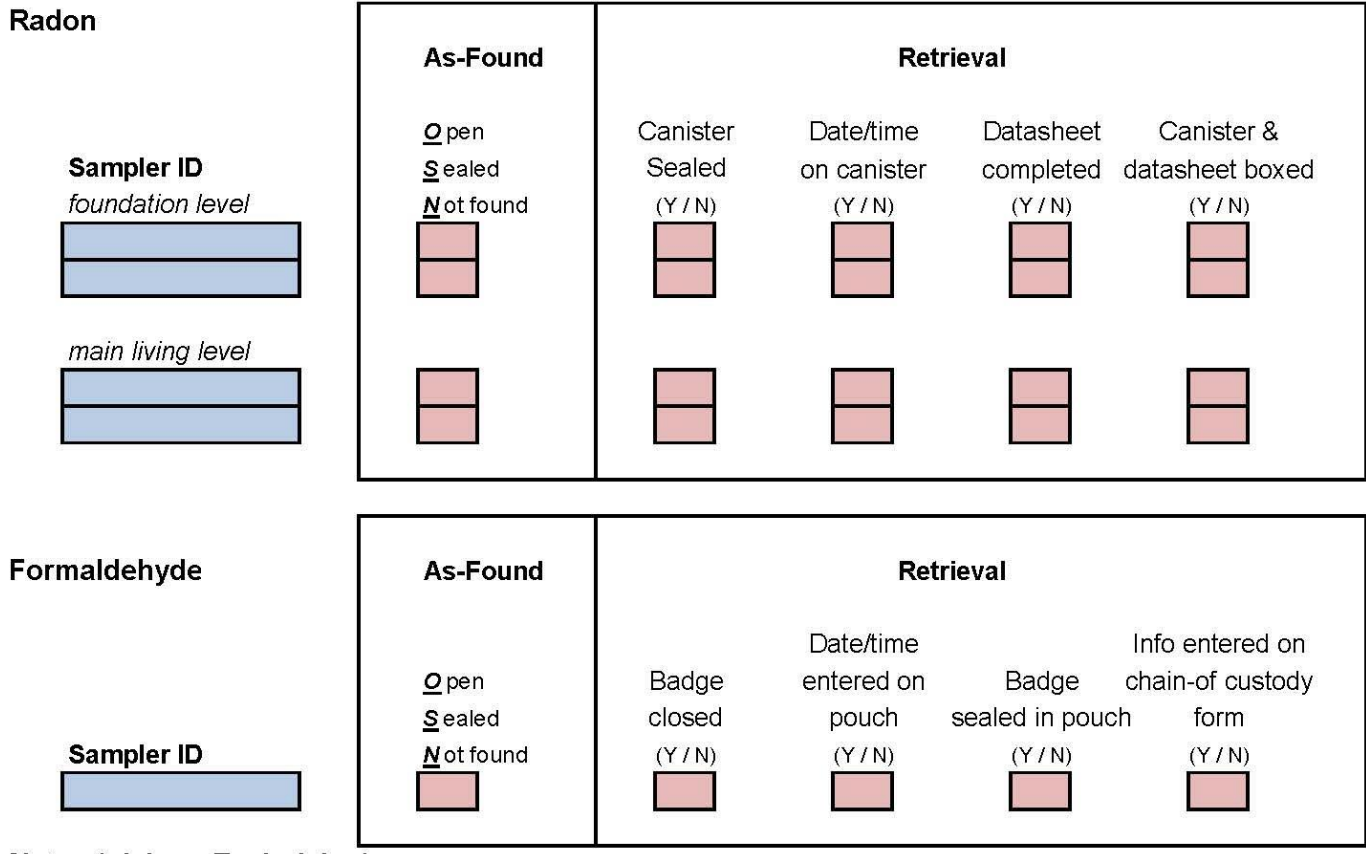

Notes (pick-up Technician): 


\section{Refrigerator Logger Retrieval}

Retrieve meters on refrigerators. (OK to leave extension cord behind.)

If not found or unable to retrieve, please call technical support (877-297-2797).

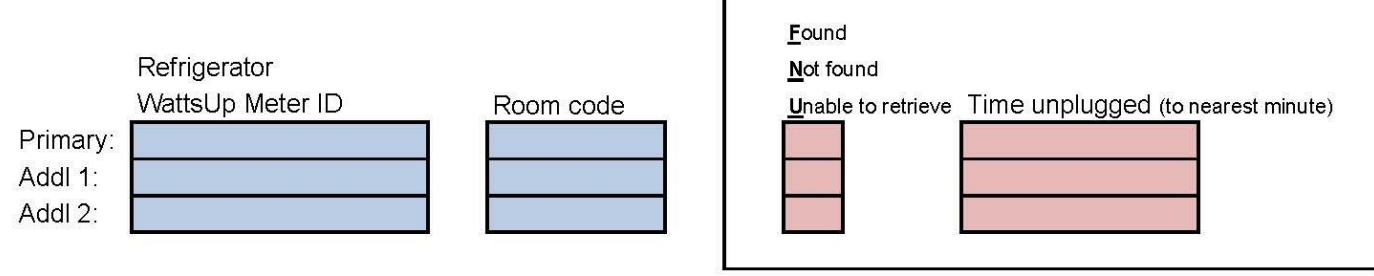

Retum refrigerator to normal position, if moved to retrieve meter. Plug refrigerator back in!

\begin{tabular}{|lll|}
\hline Room codes: & & \\
BS & basement & HL hall or mudroom \\
GA & garage & LS other living-space location* \\
PR & porch & OT other location not in living space* \\
KT & kitchen or pantry & "describe in Notes \\
\hline
\end{tabular}

Notes (Install Technician):

Notes (Pick-Up Technician):

\section{Data Logger Retrieval}

Retrieve installed data loggers and place in zip-top plastic bag for return to ECW.

Call Tech Support (877-297-2797) for further direction if you answer

" $N$ " under "Found?" in the table below or if removing any loggers

would damage the home or any homeowner property.

Temperature logger

Logger ID

(at main thermostat)

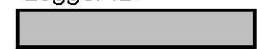

CO logger
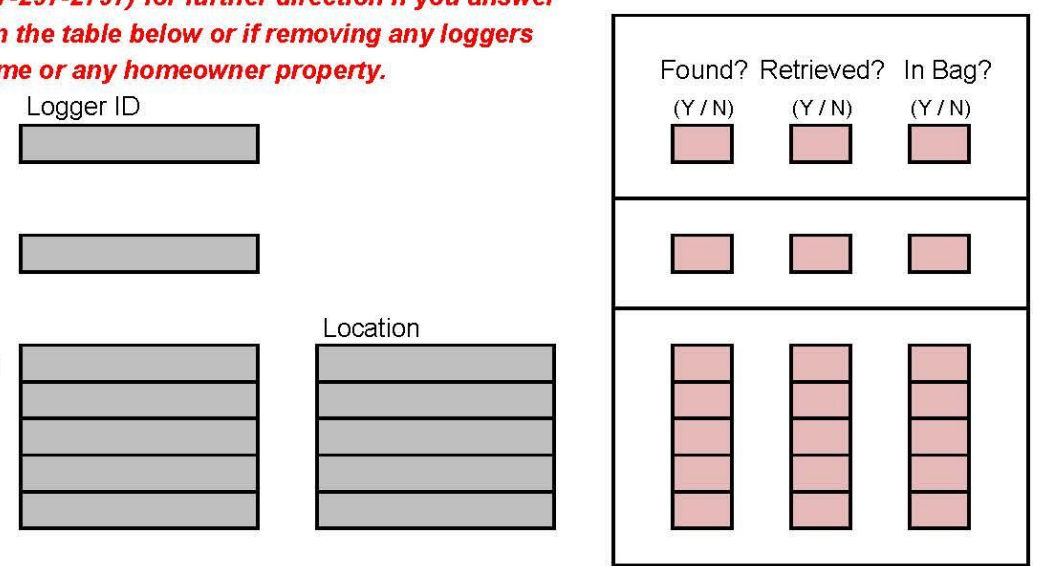
Incentive

Check or gift card provided?

$\square \underline{\text { Yes } / \text { No }}$

Final Checklist - Mark "Y"or "N" to state whether a logger was removed on pick-up. Mark "NA" if no logger was installed.

Radon / formaldehyde samplers removed from home?
Refrigerator meter(s) removed from home?
Metered refrigerator(s) in normal position and plugged in?
Temperature/humidity logger removed from home?
CO logger removed from home?
Oven / unvented heater loggers removed from home?

Notes (Pick-up Technician): 


\section{APPENDIX G. OCCUPANT SURVEY INSTRUMENT}





\section{APPENDIX G. OCCUPANT SURVEY INSTRUMENT}

\section{National Weatherization Assistance Program Evaluation Indoor Air Quality (IAQ) Occupant Survey}

\section{INTRODUCTION}

\section{[IF THIS SURVEY IS COMPLETED DURING THE VISIT]:}

(Client calls into call center, stating name, study ID and mentioning the IAQ Study)

Hello. This is (INTERVIEWER) from (PHONE CENTER). Just to confirm, you said your name is ?

S1. RECORD NAME:

S2. STUDY ID:

S3. Are you the head of household or spouse? (INTERVIEWER NOTE: if the head of household or spouse is disabled, a proxy may answer)

01 YES - CONTINUE WITH INTERVIEW

$02 \mathrm{NO}$

03 PROXY ANSWERS FOR HEAD OF HOUSEHOLD

IF S3=02:

S4. When can we schedule a time to speak with the head of household or spouse? (SCHEDULE A TIME.)

This survey will take about 30 minutes to complete. When you are finished, you will receive $\$ 10$ from the interviewer who has visited you today. As part of the National Weatherization Assistance Program Evaluation, we are speaking to clients about their experiences with the Weatherization Assistance Program and benefits they believe they have received from the program.

[NOTE: QUESTION S5 ADDED ON 1/26/11 TO ENSURE THAT RESPONDENTS REFER TO PREWEATHERIZATION CONDITIONS]

S5. Have you already received weatherization services?

01 YES

02 NO

IF S5=01 In this survey, we would like you to think back to the conditions in your home before receiving weatherization. 


\section{[IF THIS SURVEY IS COMPLETED AFTER THE VISIT]:}

(Call center calls the client)

Hello. May I please speak to ?

(IF CLIENT NOT AVAILABLE): Is there another head of household or spouse who is available?

(IF HEAD OF HOUSEHOLD OR SPOUSE NOT AVAILABLE): When would be a good time to call back?

This is (INTERVIEWER) from (PHONE CENTER), calling on behalf of the National Weatherization Assistance Program Evaluation. Your home was recently visited by a technician and field interviewer. I am calling with a follow-up survey that will take about 30 minutes to complete. In appreciation of your time, we will send you $\$ 10$ upon completion of this survey. We are speaking to clients about their experiences with the Weatherization Assistance Program and benefits they believe they have received from the program.

S1. May I please have your name?

S2. (PROGRAMMER: STUDY ID SHOULD BE LINKED TO THIS FILE AT THE TIME OF DIAL OUT)

S3. Are you the head of household or spouse? (INTERVIEWER NOTE: if the head of household or spouse is disabled, a proxy may answer)

01 YES - CONTINUE WITH INTERVIEW

02 NO

03 PROXY ANSWERS FOR HEAD OF HOUSEHOLD

IF S3=02:

S4. When can we schedule a time to speak with the head of household or spouse? (SCHEDULE A TIME.)

S5. Have you already received weatherization services?

01 YES

02 NO

IF S5=01 In this survey, we would like you to think back to the conditions in your home before receiving weatherization.

[READ IF NECESSARY. THIS IS FOR RESPONDENTS WHO ARE CURIOUS ABOUT UPCOMING APPPOINTMENTS: If you have any questions regarding your third and fourth visits during this study, please contact APPRISE at their toll-free number, 1-888-434-8008.]

\section{ANSWERING MACHINE SCRIPT:}

Hello [RESPONDENT NAME].This is (INTERVIEWER) from (PHONE CENTER), calling on behalf of the National Weatherization Assistance Program Evaluation. Your home was recently visited by a technician and field interviewer. I am calling with a follow-up survey that will take about 30 minutes to complete. In appreciation of your time, we will send you \$10 upon completion of this survey. We will call you back at a later time. However, if you prefer, you may call us at 1-800-643-2611 to complete the survey at a convenient time for you. When you call, please mention the Indoor Air Quality Study and have your study ID available. Your ID is [INSERT ID]. Thank you and have a good day.

INTERVIEWER NOTE: IF RESPONDENT CALLS IN FROM AN ANSWERING MACHINE MESSAGE, MAKE SURE TO CONFIRM THAT THE RESPONDENT NAME (FROM THE DATA FILE) LIVES IN THE HOUSEHOLD. 


\section{A. House Characteristics}

First, I have some questions about your house.

A1. Is any part of your home over a crawl space with exposed dirt as the floor?

01 YES

02 NO

96 REFUSED

97 DON'T KNOW/NOT SURE

A2. Is any part of your home over a basement?

01 YES

02 NO [SKIP TO A4]

96 REFUSED [SKIP TO A4]

97 DON'T KNOW/NOT SURE [SKIP TO A4]

A3. Do you use your basement for living space? That is, do you use it for work, play or sleep?

01 YES

02 NO

96 REFUSED

97 DON'T KNOW/NOT SURE

A4. Does your home have a garage that is attached to or part of your home?

01 YES

02 NO [SKIP TO B1]

96 REFUSED [SKIP TO B1]

97 DON'T KNOW/NOT SURE [SKIP TO B1]

A5. IF S5=01: Before weatherization, did you warm up your vehicle in your garage?

IF S5=02: Do you warm up your vehicle in your garage?

01 YES

$02 \mathrm{NO}$

96 REFUSED

97 DON'T KNOW/NOT SURE

A6. IF S5=01: Before weatherization, had you or anyone else living in your home observed the smell of vehicle exhaust inside your home?

IF S5=02: Have you or anyone else living in your home observed the smell of vehicle exhaust inside your home?

01 YES

02 NO

96 REFUSED

97 DON'T KNOW/NOT SURE

\section{B. Home Heating and Ventilation}

Now I have some questions about the heating and ventilation in your home.

B1. Let's start with the main source of heating in your home. Please tell me which type of heating equipment provides most of the heat for your home. Remember to include portable heaters, fireplaces, heating stoves and cooking stoves. (READ OPTIONS.) 
01 Heat pump

02 Central furnace with ducts to individual rooms

03 Steam/Hot water system with radiators or pipes in each room

04 Built-in electric units in each room installed in walls, ceilings, baseboards, or floors

05 Built-in floor/wall pipeless furnace

06 Built-in room heater burning gas, oil, or kerosene

07 Heating stove burning wood, coal, or coke

08 Portable heaters

09 Fireplace

10 Cooking stove used to heat your home as well as to cook

11 SOME OTHER EQUIPMENT (SPECIFY

B2. You told me that [INSERT B1] is the main source of heat in your home. In the past 12 months, did you use any other types of heating equipment? Remember to include portable heaters, fireplaces, heating stoves and cooking stoves. (READ OPTIONS.) CHECK ALL THAT APPLY

01 No other equipment

02 Heat pump

03 Central furnace with ducts to individual rooms

04 Steam/Hot water system with radiators or pipes in each room

05 Built-in electric units in each room installed in walls, ceilings, baseboards, or floors

06 Built-in floor/wall pipeless furnace

07 Built-in room heater burning gas, oil, or kerosene

08 Heating stove burning wood, coal, coke, or biomass (such as pellets or corn)

09 Portable heaters

10 Fireplace

11 Cooking stove used to heat your home as well as to cook

12 SOME OTHER EQUIPMENT (SPECIFY

B3. [IF B1=07 or B2=08] Which statement best describes your heating stove?

01 Manufactured before 1992

02 Energy Star

03 Neither

96 REFUSED

97 DON'T KNOW/NOT SURE

B4. [IF B1=09 or B2=10] Does your fireplace have a flue to the outside or is it entirely self-contained?

01 Flue to the outside

02 Flueless (self-contained)

96 REFUSED

97 DON'T KNOW/NOT SURE

B5. [IF B1=07, B1=09, B2=08, or B2=10] In the past 12 months how often did you have to burn garbage, cardboard, plastics, foam, colored ink, magazines, boxes, or wrappers to keep warm?

01 Never

02 Once

03 Sometimes

04 Often

05 Most of the winter 
96 REFUSED

97 DON'T KNOW/NOT SURE

B6. [IF B1=07, B1=09, B2=08, or B2=10] In the past 12 months how often did you have to burn coated, painted, or pressure-treated wood, driftwood, plywood, particle board, or any wood with glue in it to keep warm?

01 Never

02 Once

03 Sometimes

04 Often

05 Most of the winter

96 REFUSED

97 DON'T KNOW/NOT SURE

B7. [IF B1=07, B1=09, B2=08, or B2=10] In the past 12 months how often did you have to burn wet, rotted, diseased, or moldy wood to keep warm?

01 Never

02 Once

03 Sometimes

04 Often

05 Most of the winter

96 REFUSED

97 DON'T KNOW/NOT SURE

B8. What fuel does the cooking stove and/or oven use? (DO NOT READ) CHECK ALL THAT APPLY

01 ELECTRICITY

02 NATURAL GAS FROM UNDERGROUND PIPES

03 PROPANE (BOTTLED GAS)

04 FUEL OIL

05 KEROSENE

06 WOOD

07 SOME OTHER FUEL (SPECIFY

08 NO WORKING STOVE OR OVEN IN THE HOME

B9. IF S5=01: Before weatherization, was there an exhaust fan that vents to the outside that was regularly used when cooking in your kitchen?

IF S5=02: Is an exhaust fan that vents to the outside used regularly when cooking in your kitchen?

01 YES

02 NO

96 REFUSED

97 DON'T KNOW/NOT SURE

B10. In the past 12 months how often have you used your oven to heat your house?

01 Never

02 Rarely

03 Sometimes

04 Frequently

05 All the time 
96 REFUSED

97 DON'T KNOW/NOT SURE

B11. IF S5=01: Before weatherization, did your heating system have an air filter?

IF S5=02: Does your heating system have an air filter?

01 YES

02 NO [SKIP TO B14]

96 REFUSED [SKIP TO B14]

97 DON'T KNOW/NOT SURE [SKIP TO B14]

B12. IF S5=01: Before weatherization, was the air filter in your heating system a High Efficiency Particulate Arresting (HEPA) filter?

IF S5=02: Is the air filter in your heating system a High Efficiency Particulate Arresting (HEPA) filter?

01 YES

02 NO

96 REFUSED

97 DON'T KNOW/NOT SURE

B13. IF S5=01: Before weatherization, approximately how often did someone in your household change (or clean) the air filter in your heating system?

IF S5=02: Approximately, how often does someone in your household change (or clean) the air filter in your heating system?

01 Monthly

02 Every three months

03 Every six months

04 Once a year

05 Once every two years

06 Don't change (or clean) it

07 Air filter is changed by service company

96 REFUSED

97 DON'T KNOW/NOT SURE

B14. [IF B1=02 or B2=03] Do you know when was the last time your furnace received maintenance service by a furnace contractor to ensure optimum and safe operation (IF S5=01: before weatherization)?

01 YES

02 NO [SKIP TO B16]

96 REFUSED [SKIP TO B16]

97 DON'T KNOW/NOT SURE [SKIP TO B16]

B15. How many years and months (IF S5=01: before weatherization; IF S5=02: ago) did this occur?

B16. IF S5=01: Before weatherization, did you have a CO (or carbon monoxide) monitor in your house, other than the one that was installed by the technician as part of this study? 
IF S5=02: Do you have a CO (or carbon monoxide) monitor in your house other than the one that was installed by the technician as part of this study?

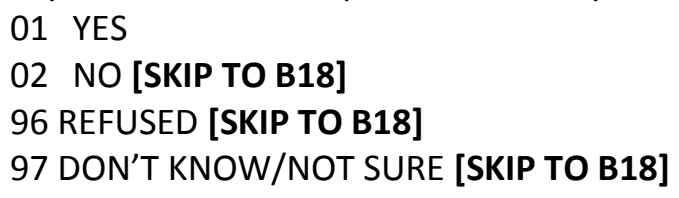

01 YES

02 NO [SKIP TO B18]

96 REFUSED [SKIP TO B18]

97 DON'T KNOW/NOT SURE [SKIP TO B18]

B17. IF S5=01: Before weatherization, was your CO monitor working?

IF S5=02: Is your CO monitor currently working?

01 YES

02 NO

96 REFUSED

97 DON'T KNOW/NOT SURE

B18. IF S5=01: Before weatherization, did you have one or more smoke detectors in your house?

IF S5=02: Do you have one or more smoke detectors in your house?

01 YES

02 NO [SKIP TO B21]

96 REFUSED [SKIP TO B21]

97 DON'T KNOW/NOT SURE [SKIP TO B21]

B19. IF S5=01: Before weatherization, how many smoke detectors were there in your house?

IF S5=02: How many smoke detectors are there in your house?

ENTER NUMBER

96 REFUSED [SKIP TO B21]

97 DON'T KNOW/NOT SURE [SKIP TO B21]

B20. IF S5=01: Before weatherization, how many of these smoke detectors were working?

IF S5=02: How many of these smoke detectors are currently working?

ENTER NUMBER

96 REFUSED

97 DON'T KNOW/NOT SURE

B21. Opening windows on opposite sides of the house to cool the indoor temperature is called natural cross ventilation. In the past 12 months, has your household used window fans to assist with natural cross ventilation in the warmer months?

01 YES

02 NO

96 REFUSED

97 DON'T KNOW/NOT SURE

B22. How often are your windows open in the summer?

01 Never

02 Rarely

03 Sometimes

04 Frequently

05 All the time

96 REFUSED

97 DON'T KNOW/NOT SURE 
B23. How often are your windows open in the winter?

01 Never

02 Rarely

03 Sometimes

04 Frequently

05 All the time

96 REFUSED

97 DON'T KNOW/NOT SURE

Now I have some questions on the indoor air temperature of your home.

B24. IF S5=01: Before weatherization, did your home have a thermostat that controlled the heating and/or cooling in your home?

IF S5=02: Does your home have a thermostat that controls the heating and/or cooling in your home?

01 YES

02 NO [SKIP TO B37]

96 REFUSED [SKIP TO B37]

97 DON'T KNOW [SKIP TO B37]

B25. What equipment does your thermostat control?

01 Central heating only

02 Central cooling only

03 Central heating and cooling

96 REFUSED

97 DON'T KNOW

B26. Some thermostats can be programmed so that the temperature changes automatically at different times of the day; for example, the heat can be automatically turned down or lowered at night when you go to bed, then automatically adjusted up again in the morning. Is the thermostat that controls your main [IF B25=01 "Central heating"; IF B25=02 "Central cooling"; IF B26=03 "Central heating and cooling"] equipment programmable?

01 YES

02 NO [SKIP TO B31]

96 REFUSED [SKIP TO B31]

97 DON'T KNOW/NOT SURE [SKIP TO B31]

B27. IF S5=01: Before weatherization, was your thermostat programmed to change the temperature at different times of the day?

IF S5=02: Is your thermostat programmed to change the temperature at different times of the day?

01 YES

02 NO [SKIP TO B29]

96 REFUSED [SKIP TO B29]

97 DON'T KNOW/NOT SURE [SKIP TO B29] 
B28. Please indicate how the programmable thermostat is used. You may select one or more of the following statements. (Select all that apply.)

01 Thermostat is automatically adjusted to a lower temperature at night during the winter

02 Thermostat is automatically adjusted to a lower temperature during the day when no one is home during the winter

03 Thermostat is automatically adjusted to a higher temperature at night during the summer

04 Thermostat is automatically adjusted to a higher temperature during the day when no one is home during the summer

05 Other

96 REFUSED

97 DON'T KNOW/NOT SURE

B29. IF S5=01: Before weatherization, how often was the current temperature setting "overridden" temporarily and why?

IF S5=02: How often is the current temperature setting "overridden" temporarily and why?

01 Daily

02 Weekly

03 Monthly

04 Every three months or so

05 Once a year

06 Less than once a year

\{IF ANSWERED ANY OF THE ABOVE\} Please explain why.

07 Never

96 REFUSED

97 DON'T KNOW/NOT SURE

B30. IF S5=01: Before weatherization, how often was the "hold" mode used and why?

IF S5=02: How often is the "hold" mode used and why?

01 Daily

02 Weekly

03 Monthly

04 Every three months or so

05 Once a year

06 Less than once a year

\{IF ANSWERED ANY OF THE ABOVE\} Please explain why.

07 Never

96 REFUSED

97 DON'T KNOW/NOT SURE

B31. IF S5=01: Before weatherization, during the winter, at what temperature was the thermostat set when someone was inside your home during the day? [IF NO ANSWER, PROBE: CAN I JUST HAVE YOUR BEST ESTIMATE?] 
IF S5=02: During the winter, at what temperature is the thermostat set when someone is inside your home during the day? [IF NO ANSWER, PROBE: CAN I JUST HAVE YOUR BEST ESTIMATE?]

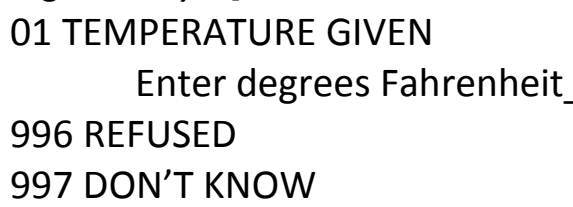

B32. IF S5=01: Before weatherization, during the winter, at what temperature was the thermostat set when no one was inside your home during the day? [IF NO ANSWER, PROBE: CAN I JUST HAVE YOUR BEST ESTIMATE?] IF S5=02: During the winter, at what temperature is the thermostat set when no one is inside your home during the day? [IF NO ANSWER, PROBE: CAN I JUST HAVE YOUR BEST ESTIMATE?]

\author{
01 TEMPERATURE GIVEN \\ Enter degrees Fahrenheit
}

996 REFUSED

997 DON'T KNOW

B33. IF S5=01: Before weatherization, during the winter, at what temperature was the thermostat set inside your home at night? [IF NO ANSWER, PROBE CAN I JUST HAVE YOUR BEST ESTIMATE?]

IF S5=02: During the winter, at what temperature is the thermostat set inside your home at night? [IF NO ANSWER, PROBE CAN I JUST HAVE YOUR BEST ESTIMATE?]

\title{
01 TEMPERATURE GIVEN \\ Enter degrees Fahrenheit
}

996 REFUSED

997 DON'T KNOW

\section{[ASK QUESTIONS B34, B35, B36 IF B25=02 OR B25=03]}

Now I would like you to think about the temperature inside your home when using your central air conditioning equipment last summer. For the next questions, if the thermostats are set at different temperatures, only report for the thermostat that affects the rooms where most of the people are.]

B34. During the summer, at what temperature is the central air conditioning equipment set when someone is inside your home during the day? IF NO ANSWER, PROBE WHAT'S YOUR BEST ESTIMATE?

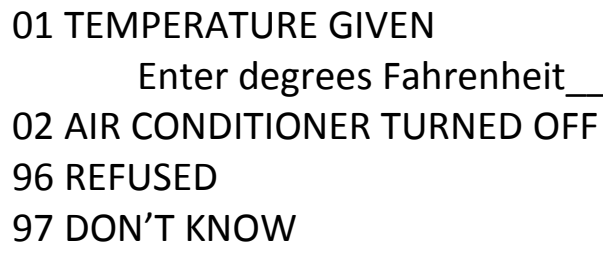


B35. During the summer, at what temperature is the central air conditioning equipment set when no one is inside your home during the day? IF NO ANSWER, PROBE WHAT'S YOUR BEST ESTIMATE?

\author{
01 TEMPERATURE GIVEN \\ Enter degrees Fahrenheit \\ 02 AIR CONDITIONER TURNED OFF \\ 96 REFUSED \\ 97 DON'T KNOW
}

B36. During the summer, at what temperature is the central air conditioning equipment set inside your home at night? IF NO ANSWER, PROBE WHAT'S YOUR BEST ESTIMATE?

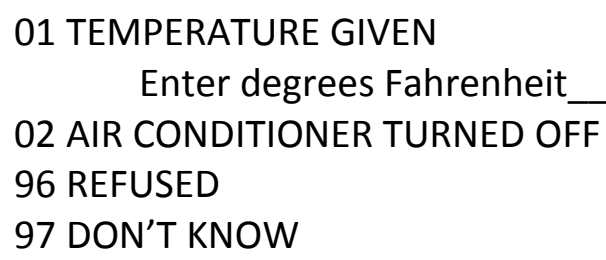

B37. In the past 12 months, was your household unable to use any of the following equipment because it was broken? CHECK ALL THAT APPLY
01 Main Heating Equipment
02 Central Air Conditioner
03 Room Air Conditioner
04 NO EQUIPMENT BROKEN
96 REFUSED
97 DON'T KNOW/NOT SURE

B38. Which of the following statements best describes the indoor temperature of your home (IF S5=01: before weatherization) during the winter:
01 Very cold
02 Cold
03 Comfortable
04 Hot
05 Very hot
06 Other
96 REFUSED
97 DON'T KNOW/NOT SURE

B39. Which of the following statements best describes the indoor temperature of your home during the summer:

01 Very cold

02 Cold

03 Comfortable 
$04 \mathrm{Hot}$

05 Very hot

06 Other

96 REFUSED

97 DON'T KNOW/NOT SURE

B40. In the past 12 months, has a landlord controlled the temperature inside your home?

01 YES

$02 \mathrm{NO}$

03 DO NOT HAVE LANDLORD

96 REFUSED

97 DON'T KNOW/NOT SURE

B41. In the past 12 months how often did your household keep your home at a temperature that you felt was unsafe or unhealthy? (READ)

01 Almost every month

02 Some months

031 or 2 months

04 Never

96 REFUSED

97 DON'T KNOW/NOT SURE

B42. In the past 12 months, has anyone in the household needed medical attention because your home was too cold?

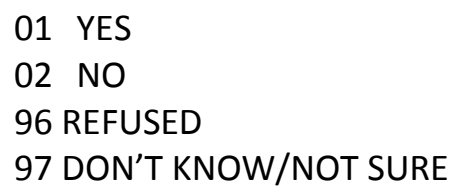

B43. In the past 12 months did anyone in your household need medical attention because your home was too hot?

01 YES

02 NO

96 REFUSED

97 DON'T KNOW/NOT SURE

B44. During the past 12 months, how often have you or other members of your household found your home too drafty? Would you say it is. . (READ)

01 All the time,

02 Most of the time,

03 Some of the time, or

04 Never

96 REFUSED

97 DON'T KNOW/NOT SURE

B45. IF S5=01: Before weatherization, did your clothes dryer vent directly to the outdoors? IF S5=02: Does your clothes dryer vent directly to the outdoors?

01 YES 
02 NO

03 DRYER IS VENTLESS

04 DON'T HAVE CLOTHES DRYER

96 REFUSED

97 DON'T KNOW/NOT SURE

B46. IF S5=01: Before weatherization, did your main bathroom have a ventilation fan in it that worked? IF S5=02: Does your main bathroom have a ventilation fan in it that works?

01 YES

02 NO [SKIP TO B49]

96 REFUSED

97 DON'T KNOW/NOT SURE

B47. IF S5=01: Before weatherization, how often did you or members of your household operate the fan while showering? (READ)

IF S5=02: How often do you or members of your household operate the fan while showering? (READ)

01 Never [SKIP TO B49]

02 Rarely

03 Sometimes

04 Frequently

05 All the time

96 REFUSED [SKIP TO B49]

97 DON'T KNOW/NOT SURE

B48. IF S5=01: Before weatherization, how long after showering did you or members of your household operate the fan?

IF S5=02: How long after showering do you or members of your household operate the fan?

01 Don't turn the fan on for showers

02 The fan is turned off when leaving the shower area

03 A few minutes

04 Several minutes

05 Until the steam in the shower area is gone

96 REFUSED

97 DON'T KNOW/NOT SURE

B49. Electric dehumidifiers remove moisture from the air and are often used in the summer. (IF

$\mathrm{S} 5=01$ : Before weatherization, was; IF S5=02: Is) a dehumidifier used in your home?

01 YES

02 NO [SKIP to SECTION C]

96 REFUSED [SKIP TO SECTION C]

\section{DON'T KNOW/NOT SURE [SKIP TO SECTION C]}

B50. In the past 12 months, how many months was the dehumidifier used? (READ)

011 to 3 months

024 to 6 months,

037 to 9 months,

0410 to 11 months, but not all year, or is it

05 Turned on all year long? 
96 REFUSED

97 DON'T KNOW/NOT SURE

\section{Home Conditions}

Now we have a few questions about the condition of your home.

C1. IF S5=01: Before weatherization, how infested was your home with cockroaches or other insects or spiders?

IF S5=02: How infested is your home with cockroaches or other insects or spiders?

01 Extremely infested

02 Very infested

03 Somewhat infested

04 Hardly infested

05 Not infested at all [SKIP TO C3]

96 REFUSED [SKIP TO C3]

97 DON'T KNOW/NOT SURE [SKIP TO C3]

C2. What have you done about the cockroaches, other insects or spiders? (DO NOT READ. CHECK ALL

THAT APPLY.)

01 NOTHING

02 USED INSECTICIDES, BUG SPRAYS, OR POISON

03 HIRED AN EXTERMINATOR OR OTHER PROFESSIONAL

04 OTHER. PLEASE SPECIFY.

96 REFUSED

97 DON'T KNOW/NOT SURE

C3. IF S5=01: Before weatherization, how infested was your home with rats or mice?

IF S5=02: How infested is your home with rats or mice?

01 Extremely infested

02 Very infested

03 Somewhat infested

04 Hardly infested

05 Not infested at all [SKIP TO C5]

96 REFUSED [SKIP TO C5]

97 DON'T KNOW/NOT SURE [SKIP TO C5]

C4. What have you done about the pests? (DO NOT READ. CHECK ALL THAT APPLY.)

01 NOTHING

02 USED BAIT OR POISON

03 HIRED AN EXTERMINATOR OR OTHER PROFESSIONAL

04 OTHER. PLEASE SPECIFY.

96 REFUSED

97 DON'T KNOW/NOT SURE 
C5. IF S5=01: Before weatherization, did your home frequently have a mildew odor or musty smell? IF S5=02: Does your home frequently have a mildew odor or musty smell?

01 YES

02 NO

96 REFUSED

97 DON'T KNOW/NOT SURE

C6. IF S5=01: Before weatherization, how often did you observe standing water anywhere in your home? (READ) (IF NECESSARY: Examples of standing water include wet carpet, puddles, or flooding in the home.)

IF S5=02: How often do you observe standing water anywhere in your home? (READ) (IF NECESSARY: Examples of standing water include wet carpet, puddles, or flooding in the home.)

01 Never

02 Rarely

03 Sometimes

04 Often

05 Always

96 REFUSED

97 DON'T KNOW/NOT SURE

C7. Have you seen mold in your home in the past 12 months?

01 YES

02 NO

96 REFUSED

97 DON'T KNOW/NOT SURE

[ASK IF C7=01]

C8. What have you done about the mold? (DO NOT READ. CHECK ALL THAT APPLY.)

01 NOTHING

02 CLEANED WITH BLEACH

03 CLEANED WITH OTHER CHEMICAL MOLD REMOVER

04 CLEANED WITH NATURAL MOLD REMOVER (VINEGAR OR NATURAL PRODUCT)

05 AIR CONDITIONED

06 VENTILATION (FANS)

07 USED A DEHUMIDIFIER

08 CONTACTED A PROFESSIONAL

09 OTHER. PLEASE SPECIFY

96 REFUSED

97 DON'T KNOW/NOT SURE

Now I have some questions about paying energy bills.

C9. In the past 12 months was your electricity or natural gas ever disconnected because you were unable to pay your home energy bill?

01 YES

$02 \mathrm{NO}$ 


\section{REFUSED}

\section{DON'T KNOW/NOT SURE}

\section{[ASK IF C9=01]}

C10. While your electricity or natural gas was disconnected, was there a time when you wanted to use your main source of heat but were unable to?

01 YES

02 NO

96 REFUSED

\section{DON'T KNOW/NOT SURE}

\section{[ASK IF C9=01]}

C11. If your electricity was disconnected, was there a time when you wanted to use your air conditioner but were unable to?

01 YES

02 NO

96 REFUSED

97 DON'T KNOW/NOT SURE

C12. In the past 12 months did your fuel oil, kerosene, propane, or wood ever run out because you were unable to pay for a home energy delivery?

01 YES

02 NO

96 REFUSED

97 DON'T KNOW/NOT SURE

\section{Health Care and Coverage}

The next set of questions is about health care and coverage.

D1. In the past 12 months have you had any kind of health care coverage, including health insurance, prepaid plans such as HMOs, or government plans such as Medicare?

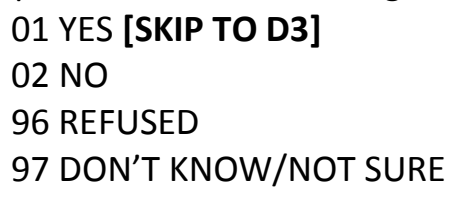

\section{[ASK IF D1=02, 96, 97]}

D2. According to the information given, you do NOT have health care coverage of any kind. Do you have health insurance or coverage through a plan I might have missed? (INTERVIEWER: REVIEW PLANS IF INFORMANT IS UNSURE.) CHECK ALL THAT APPLY.

01 NO/NOT COVERED BY ANY PLAN

02 HEALTH INSURANCE PLAN FROM A CURRENT OR PAST EMPLOYER/UNION/SCHOOL

03 A HEALTH INSURANCE PLAN BOUGHT ON HIS/HER OWN/PROF. ASSN

04 A PLAN BOUGHT BY SOMEONE WHO DOES NOT LIVE IN THIS HOUSEHOLD

05 MEDICARE

06 MEDICAID/STATE NAME 
07 CHAMPUS/CHAMP-VA, TRICARE, VA,

10 OTHER MILITARY

11 INDIAN HEALTH SERVICE

12 [fill STATE PLAN]

13 OTHER PLAN [SPECIFY]

96 REFUSED

97 DON'T KNOW/NOT SURE

\section{[ASK IF D1=01]}

D3. During the past 12 months was there any time that you did not have any health insurance coverage?

01 YES

02 NO

96 REFUSED

97 DON'T KNOW/NOT SURE

Now I am going to read some statements about health and medical care.

D4. Usually, you go to the doctor as soon as you start to feel bad. Is that:

01 definitely true

02 mostly true

03 mostly false, or

04 definitely false?

96 REFUSED

97 DON'T KNOW/NOT SURE

D5. You will do just about anything to avoid going to the doctor. Is that ...

01 definitely true

02 mostly true

03 mostly false, or

04 definitely false?

96 REFUSED

97 DON'T KNOW/NOT SURE

\section{E. Health}

For this section, I will be asking health related questions.

E1. Now thinking about physical health, which includes physical illness and injury, for how many days during the (IF S5=01: 30 days before weatherization; IF S5=02: past 30 days) was your physical health not good?

01 NUMBER OF DAYS

02 NONE

96 REFUSED

97 DON'T KNOW/NOT SURE

E2. Now thinking about your mental health, which includes stress, depression, and problems with emotions, for how many days during the (IF S5=01: 30 days before weatherization; IF $\mathrm{S} 5=02$ : past 30 days) was your mental health not good?

01 NUMBER OF DAYS

02 NONE 
96 REFUSED

97 DON'T KNOW/NOT SURE

E3. (IF S5=01: During the 30 days before weatherization; IF S5=02: During the past 30 days), for about how many days have you felt you did not get enough rest or sleep?
01 NUMBER OF DAYS

02 NONE

96 REFUSED

97 DON'T KNOW/NOT SURE

E4. (IF S5=01: During the 30 days before weatherization; IF S5=02: During the past 30 days), for about how many days have you felt very healthy and full of energy?

01 NUMBER OF DAYS

02 NONE

96 REFUSED

97 DON'T KNOW/NOT SURE

E5.(IF S5=01: During the 30 days before weatherization; IF S5=02: During the past 30 days), for about how many days did poor physical or mental health keep you from doing your usual activities, such as self-care, work, or recreation?

01 NUMBER OF DAYS

02 NONE

96 REFUSED

97 DON'T KNOW/NOT SURE

Next, I am going to ask you whether you have had some particular health

problems in the (IF S5=01: 3 months before weatherization; IF S5=02: last 3 months). In the (IF $S 5=01: 3$ months before weatherization: IF S5=02: past 3 months), have you had ...

E6. Shortness of breath when lying down, waking up, or with light work or light exercise?

01 YES

02 NO

96 REFUSED

97 DON'T KNOW/NOT SURE

E7. Headaches that (IF S5=01: were; IF S5=02: are) either new or more frequent or severe than ones you (IF S5=01: had; IF S5=02: have had) before?

01 YES

02 NO

96 REFUSED

97 DON'T KNOW/NOT SURE

Now I am going to ask whether members of your household have had particular health problems.

E8. Including yourself, how many people normally live in this household? Do not include anyone who is just visiting, those away in the military, or children who are away at college. 


\section{ENTER NUMBER}

E9. Can you please tell me their first names, gender and age, your relationship to the person, and whether or not they are in school? Please start with yourself. [IF E8=1: "Can you tell me your name again, as well as your age and whether or not you are in school."]

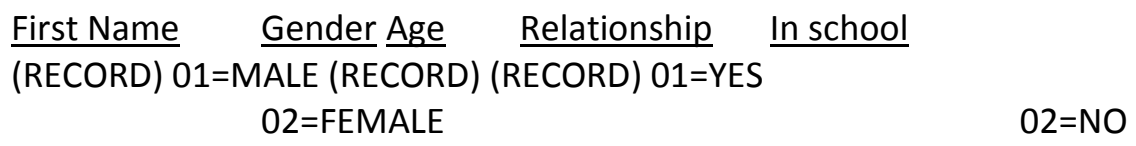

Respondent Self

Person 2.

Person 3.

Person 4.

Person 5.

Person 6.

Person 7.

Person 8.

Person 9.

Person 10.

In the past 12 months were you or anyone else in the household ever told by a doctor or health professional that you or they have.. CHECK ALL THAT APPLY

E10. Lead poisoning

01 YES

02 NO

96 REFUSED

97 DON'T KNOW/NOT SURE

[IF E10=01] Please list all individuals, including yourself:

E11. Three or more ear infections per year

01 YES

02 NO

96 REFUSED

97 DON'T KNOW/NOT SURE

[IF E11=01] Please list all individuals, including yourself: 
E12. Any kind of respiratory allergy

01 YES

02 NO

96 REFUSED

97 DON'T KNOW/NOT SURE

[IF E12=01] Please list all individuals, including yourself:

E13. Flu

01 YES

02 NO

96 REFUSED

97 DON'T KNOW/NOT SURE

[IF E13=01] Please list all individuals, including yourself:

E14. Persistent Cold symptoms lasting more than 14 days (SYMPTOMS INCLUDE COUGHING, SORE THROAT, SNEEZING, SINUS PAIN, CONGESTION, FEVER, FATIGUE, AND HEADACHE)

01 YES

02 NO

96 REFUSED

97 DON'T KNOW/NOT SURE

[IF E14=01] Please list all individuals, including yourself:

E15. Sinus infection or Sinusitis

01 YES

02 NO

96 REFUSED

97 DON'T KNOW/NOT SURE

[IF E15=01] Please list all individuals, including yourself:

E16. Bronchitis

01 YES

02 NO 
96 REFUSED

97 DON'T KNOW/NOT SURE

[IF E16=01] Please list all individuals, including yourself:

E17. Have you ever been told by a doctor or other health professional that you have asthma?

01 YES

02 NO [SKIP TO E23]

96 REFUSED [SKIP TO E23]

97 DON'T KNOW/NOT SURE [SKIP TO E23]

E18. Do you still have asthma?

01 YES

$02 N O$

96 REFUSED

97 DON'T KNOW/NOT SURE

E19. During the past 12 months, how many times did you see a doctor or health professional for a routine checkup for your asthma?

READ: Symptoms of asthma include coughing, wheezing, shortness of breath, chest tightness or phlegm production when you have a cold or respiratory infection.

E20. How long has it been since you last had any symptoms of asthma?

01 Never

02 Less than one day ago

03 1-6 Days ago

041 week to less than 3 months ago

053 months to less than 1 year ago

061 year to less than 3 years ago

073 years to 5 years ago

08 More than 5 years ago

96 REFUSED

97 DON'T KNOW/NOT SURE

E21. During the past 12 months did you have to stay overnight in the hospital because of asthma?

01 YES

02 NO

96 REFUSED

97 DON'T KNOW/NOT SURE

E22. Not counting hospitalizations, during the past 12 months, did you go to an emergency room because of asthma?

01 YES

02 NO

96 REFUSED

97 DON'T KNOW/NOT SURE 
These next questions are about cigarette smoking.

E23. Which one of the following statements best describes the rules about smoking in your home...

01 No one is allowed to smoke anywhere inside your home

02 Smoking is allowed at some places or at sometimes

03 Smoking is permitted anywhere

96 REFUSED

97 DON'T KNOW/NOT SURE

E24. Have you smoked at least 100 cigarettes in your entire life?

01 YES

02 NO

96 REFUSED

97 DON'T KNOW/NOT SURE

E25. Do you now smoke cigarettes every day, some days or not at all?

01 Everyday

02 Some days

03 Not at all

96 REFUSED

97 DON'T KNOW/NOT SURE

E26. In the past 12 months has anyone in the household been food poisoned from eating food inside your home and therefore went to see a medical professional?

01 YES

02 NO

96 REFUSED

97 DON'T KNOW/NOT SURE

E27. In the past 12 months, has anyone in the household been poisoned by breathing in carbon monoxide, and therefore went to see a medical professional?

01 YES

02 NO

96 REFUSED

97 DON'T KNOW/NOT SURE

E28. In the past 12 months, has anyone in the home been burned from scalding hot water coming out of a faucet or showerhead in your home?

01 YES

02 NO

96 REFUSED

97 DON'T KNOW/NOT SURE

\section{F. Employment}

In this section I will be asking employment and school related questions. 


\section{[SKIP IF E8=1]}

F1. Are you the primary wage earner in your household?

01 YES

02 NO

03 NO PRIMARY WAGE EARNER IN THE HOUSEHOLD

96 REFUSED

F2. [IF F1=01 or F1=03 or E8=1: Are you; IF F1=02 or F1=96: Is the primary wage earner in the household] currently...?

01 Employed for wages

02 Self-employed

03 Out of work for more than 1 year

04 Out of work for less than 1 year

05 A Homemaker

06 A Student

07 Retired

08 Unable to work

96 REFUSED

97 DON'T KNOW/NOT SURE

F3. Does a physical, mental or emotional problem NOW keep [IF F1=01 or F1=03: you; IF F1=02 or F1=96: the primary wage earner] from working at a job or business?

01 YES

02 NO

96 REFUSED

97 DON'T KNOW/NOT SURE

F4. [IF F2=01 or F2=02] In the past 12 months, about how many days of work did [if F1=01: you; if F1=02 or F1=96: the primary wage earner] miss at a job or business because of illness or injury (DO NOT INCLUDE MATERNITY LEAVE)

\section{RESPONDENT GAVE NUMBER ENTER NUMBER \\ 02 NONE \\ 96 REFUSED \\ 97 DON'T KNOW/NOT SURE}

\section{[SKIP IF E8=1]}

\section{[IF F2=01 or F2=02]}

F5. In the past 12 months, about how many days of work did [IF F1=01: you; IF F1=02 or F1=96: the primary wage earner] miss because of illness or injury of another household member?

01 RESPONDENT GAVE NUMBER

ENTER NUMBER

02 NONE

96 REFUSED

97 DON'T KNOW/NOT SURE 
[ASK IF RESPONDENT IS IN SCHOOL (FROM E9)]

F6. In the past 12 months, about how many days of school have you missed because of illness or injury?

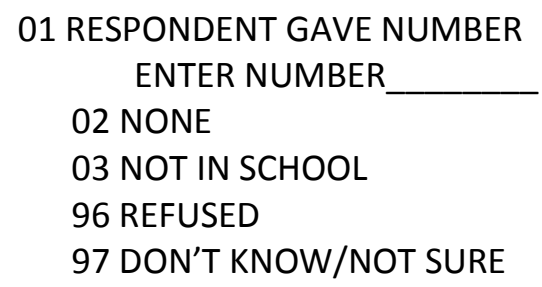

[SKIP IF E8=1]

[ASK IF F1=02 or $F 1=96$ ]

F7. And, in the past 12 months, about how many days of school has the primary wage earner missed because of illness or injury?

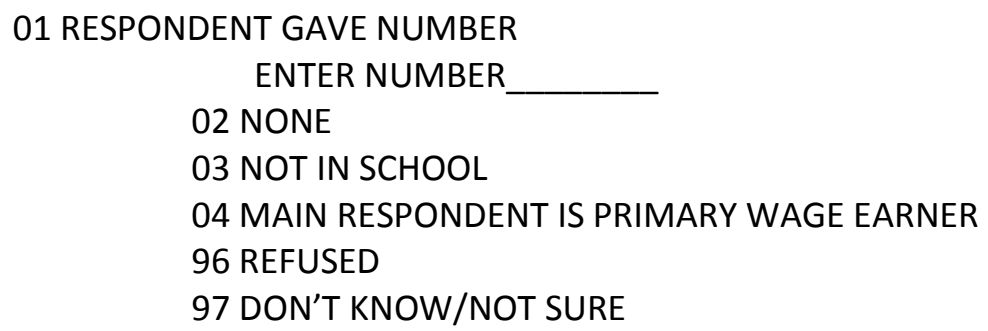

F8. Please tell us about the pre-school aged child who has missed the most school days due to illness or injury in the past 12 months. How many days of school did that child miss?

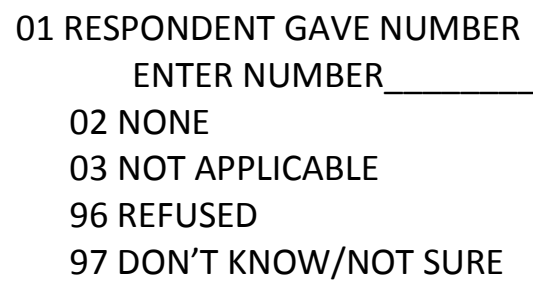

\section{[ASK IF AT LEAST ONE MEMBER OF HOUSEHOLD IS 5 TO 18-YEARS-OLD (FROM E9) AND IS IN SCHOOL (FROM E9)]}

F9. Please tell us about the school aged child who has missed the most school days due to illness or injury in the past 12 months. How many days of school did that child miss?

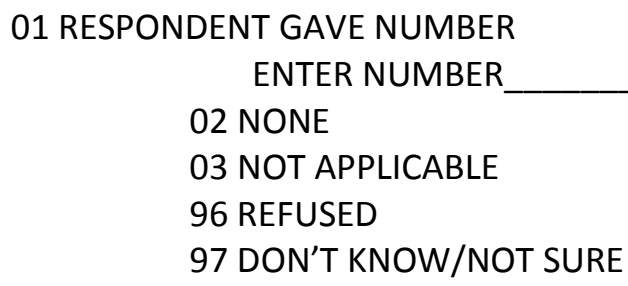




\section{G. Demographics}

Finally, I have some questions about your household.

G1. How long have you lived in your current home?

\{If less than one year\} ENTER: MONTHS

ENTER: YEARS

G2. On a typical week day is there someone at home most or all of the day?

01 YES

02 NO

96 REFUSED

97 DON'T KNOW/NOT SURE

G3. Do you rent or own your current residence?

01 RENT

02 OWN

03 NEITHER (PLEASE DESCRIBE THE HOUSING AGREEMENT).

\section{REFUSED}

\section{DON'T KNOW/NOT SURE}

G4. Which of the following best describes the location of your home? Do you live in a city, a town, the suburbs, or in a rural area?
01 City
02 Town
03 Suburbs
04 Rural

96 REFUSED

97 DON'T KNOW/NOT SURE

G5. In the past 12 months has anyone in your household owned or had the regular use of any cars, trucks, vans, sports-utility-vehicles or similar vehicles? DO NOT INCLUDE MOTORCYCLES OR MOPEDS.

01 YES

02 NO

96 REFUSED

97 DON'T KNOW/NOT SURE

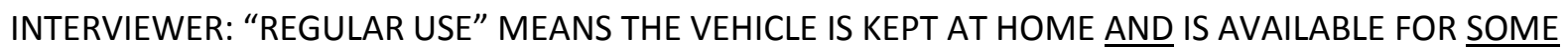
PERSONAL USE.

G6. This next question is about public transportation. Thinking of the area where you live, in the past 12 months, have members of your household had regular access to public transportation? Remember to include access to buses, trolley buses, trains, trams, rapid transit (metro/subway/underground), water taxi/ferries, free transportation offered by community services agencies, and Medicaid covered transportation.)

01 YES 
02 NO

96 REFUSED

97 DON'T KNOW/NOT SURE

Now I have several more demographic questions about you.

G7. What is the highest degree or level of school you have completed? (DO NOT READ)

01 NO SCHOOLING COMPLETED

02 KINDERGARTEN TO GRADE 12 (NO DIPLOMA)

03 HIGH SCHOOL DIPLOMA OR GED

04 SOME COLLEGE, NO DEGREE

05 ASSOCIATE'S DEGREE (FOR EXAMPLE: AA, AS)

06 BACHELOR'S DEGREE (FOR EXAMPLE: BA, BS)

07 MASTER'S DEGREE (FOR EXAMPLE: MA, MS, MBA)

08 PROFESSIONAL DEGREE (FOR EXAMPLE: MD, JD)

09 DOCTORATE DEGREE (FOR EXAMPLE: PHD, EDD)

\section{REFUSED}

97 DON'T KNOW/NOT SURE

G8. Do you consider yourself to be of Hispanic or Latino origin, such as Mexican, Puerto Rican, Cuban, or other Spanish background?

01 YES

02 NO

96 REFUSED

97 DON'T KNOW/NOT SURE

G9. Which describes your race? You can select one or more categories. (READ CHOICES)

01 White

02 Black or African-American

03 American Indian or Alaska Native

04 Asian

05 Native Hawaiian or Other Pacific Islander

06 OTHER (IF VOLUNTEERED)

07 HISPANIC OR LATINO (IF VOLUNTEERED)

96 REFUSED

97 DON'T KNOW/NOT SURE

\section{[ASK IF more than one entry in G9]}

G10. Which ONE of these groups best represents your race?

01 White

02 Black or African-American

03 American Indian or Alaska Native

04 Asian

05 Native Hawaiian or Other Pacific Islander

06 OTHER (IF VOLUNTEERED)

07 HISPANIC OR LATINO (IF VOLUNTEERED)

96 REFUSED 


\section{DON'T KNOW/NOT SURE}

That's the end of the survey. Thank you for your participation, and have a good day. 

APPENDIX H. LOCAL WEATHERIZATION AGENCY SCRIPTS AND FORMS 



\section{APPENDIX H. LOCAL WEATHERIZATION AGENCY SCRIPTS AND FORMS}

Rev. $11 / 02 / 2010$ am

\section{INTRO CALL SCRIPT - WAP Indoor Air Quality Study}

++ Good morning/afternoon. Thank you again for working with us on the WAP Indoor Air Quality study. After we introduce ourselves, the purpose of this call is to make sure everyone understands study details and to work out logistics.

Introductions

++ Has everyone had a chance to watch the video? __ Y/N _ Do you have any questions? If not, encourage watching it after the call. It covers:

Why studies are important

- Study criteria

- Study logistics

- What we need from participating agencies

\section{Brief study overview}

++ This portion of the WAP evaluation looks at how weatherization affects indoor air quality in singlefamily homes. Specifically, we are looking at radon, carbon monoxide, formaldehyde and moisture.

++ We are seeking to understand the typical impacts on indoor air quality. Data will be aggregated across agencies, states and regions - participating agencies will not be identified.

++ The evaluation team will do almost all of the legwork for these studies, including recruiting and scheduling the homes, installing and removing the metering equipment, and analyzing the results. We are asking several things of agencies: 1) a list of candidate homes; and 2) we ask that you weatherize the eight study homes within time frame of the study; and finally, provide us some follow-up information on the homes after weatherization is complete.

\section{Study Criteria}

++ We are looking for about 8 single family or mobile homes that are in your pipeline for weatherization this summer. Ideally these homes have been audited.

++ Five houses will serve as the treatment group - they will be weatherized between two sets monitoring visits by our technicians. Three houses will serve as the control group - they will be weatherized after both sets of monitoring visits.

++ The first technician visit is a couple hours long because before he installs monitoring equipment and performs standard tests

\{blower door w/ zone pressure diagnostics, heating system efficiency test (SSE), worst case draft test, CO readings $\{$ WHY? $\}$ We repeat a number of tests that agencies typically perform so that we can have a consistent dataset of pre- and post-weatherization conditions across all homes participating in the study. 
Rev. 11/02/2010 am

++ WAP clients will receive at least $\$ 120$ for participating (check or Visa gift card):

- $\$ 50$ at the time of the first visit by the field team.

- $\$ 10$ at the time of the first pick-up visit by the field team.

- $\$ 50$ at the time of the second main visit by the field team.

- $\$ 10$ at the time of the second pick-up visit by the field team.

- Control homes will receive an additional $\$ 50$ because their weatherization will happen a bit later than that of treatment homes.

++ Do you have any questions?

\section{Agency Throughput}

++ To recruit the 8 houses, we are asking you for a list of 24 houses. Do you have enough volume to handle that?

++ What is your monthly production?

++ About how many homes do you have in queue?

\section{Study Timing}

We have techs scheduled for your area the week of

++ This would mean your window for weatherizing the five treatment homes is

++ And weatherizing three control homes is

Can you work with this schedule?

\section{CANDIDATE HOMES LIST DETAILS}

1. When do you expect to send the List of Candidate Homes?

2. Who will be sending the list?

3. Does your agency prefer to contact the homeowner yourself before we begin the scheduling calls? [preferable but not essential].

4. If you prefer to notify the homeowner first, do you plan to call or send a letter? [We can send talking points and a letter template]

5. If any of the homeowners want to speak with someone at the agency to make sure our study is legit, who should they call? [Request name and phone number].

Name:

Phone \#:

6. Does your weatherization program have a local name that is different than the name of your agency?

++ Do you want someone from your agency to accompany our field tech on any of the visits?

++ [If so,] Who? 
Rev. 11/02/2010 am

\section{Additional Questions}

1) Is your agency primarily contractor or crew based?

2) What is your average job length - after audit but including inspection and reworks?

\section{Next steps}

++ I will be looking for your list of candidate homes on [DATE]

++ [If agency wants to contact candidates first] You [or we] will contact the homes to alert them that we may be calling in the next week or so.

++ We will give you the dates our technicians will be visiting the homes.

++ We will also alert you once our technicians have visited the homes a second time, and will confirm the weatherization schedule for the five treatment homes.

\section{Wrap- UP}

++ Thank you again for participating in the WAP Indoor Air Quality Study. Good-bye.

[COMPLETE WORKSHEET DURING INTRO CALL]

\section{AGENCY THROUGHPUT}

1. How many homes does your agency expect to weatherize this year?

2. How many homes does your agency currently have in the pipeline? In other words, audit is complete but home is not yet weatherized.

3. Approx. what percentage of the homes you weatherize are single family (detached, attached or mobile home)?

\section{WEATHERIZATION PROCESS}

1. How long is your typical weatherization process (from audit through post inspection?)

2. How long does weatherization work typically take (from start of work through post-inspection)?

3. Do you have any concerns about the timeframe for the IAQ study?

[Refer to master fieldwork schedule for date ranges applicable to Nov, Dec and Jan SuperPUMAS]

- Treatment group: Weatherization begins in ___and is completed within _ weeks

- Control group: Weatherization begins in ___ after final site visit has been completed

4. What type of weatherization work is done by your agency's in-house crew?

5. What type of weatherization work is done by outside contractors hired by your agency? 
National WAP Evaluation

List of Candidate Homes

Indoor Air Quality Study

Please list up to 24 homes that meet the following criteria

(1) occupied single familly home site-built detached, site-bultt attached or mobile home)

(2) weatherization work has not been started yet

作

(5) if a rental, a signed landlord agreement must be in place

and 3 control homes after

If your agency has more than 24 homes that meet these criteria, please provide information on the 24 homes that were audited most recently.

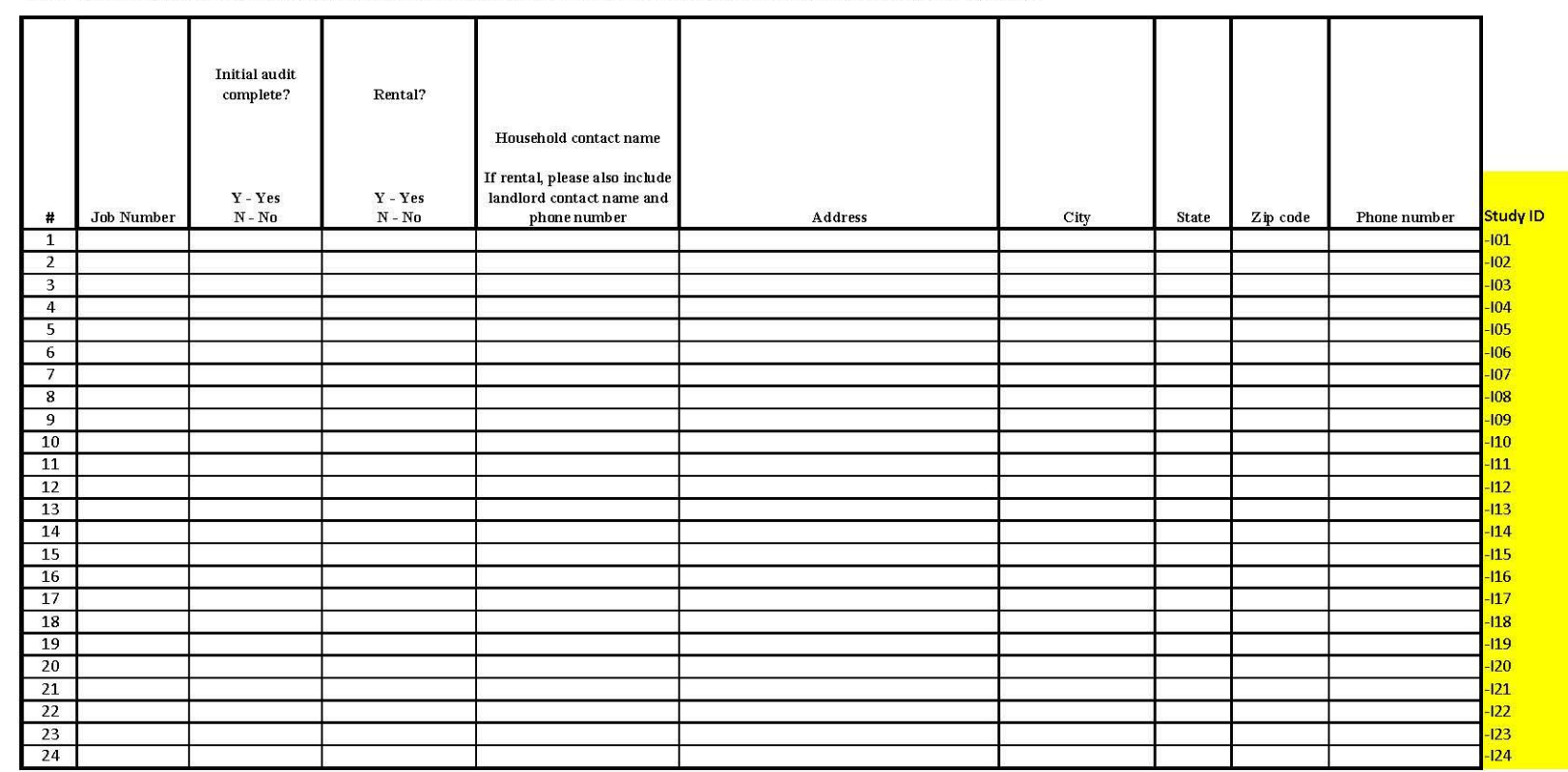

All of the information obtained from this survey will be protected and will remain confidential. The data will be analyzed in such a way that the information provided cannot be associated back to your state vour agencies, or the housing units and dients that your state served 


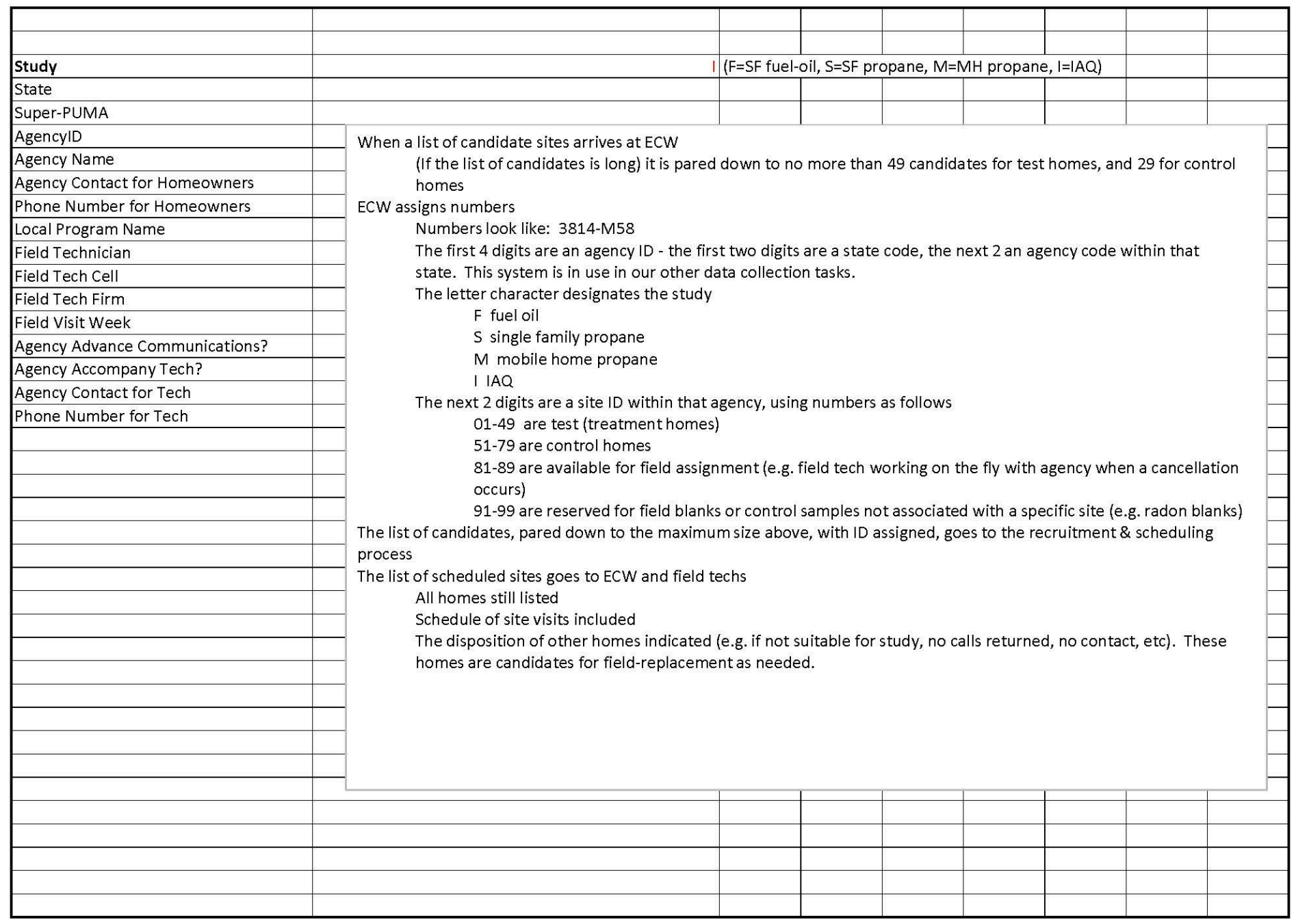


Dear,

Good afternoon. We have selected the 6 state candidates that will be participating in the indoor air quality field study; the schedule for your study participants' first technician visits is below.

Thank you again for helping with this stage of the study.

- The treatment group candidates (the four homes to be weatherized between July 5 and July 29) are shaded bluish green in the schedule. We need you to hold all weatherization for these four homes until this window.

- The control group candidates (the two homes to be weatherized after Aug 29) are shaded $\mathrm{red} / \mathrm{pink}$. We need you to hold all weatherization for these two homes until this time.

- Thank you for your willingness to accommodate these schedules. If anything comes up that affects these plans and dates (e.g. emergency furnace replacements) please let me know.

- [IF APPLICABLE] We also recruited an alternate to use in case of last minute cancellations. Please hold off work at the alternate's home as well until we have successfully been able to complete our first monitoring visits with study participants.

The name and phone number of the technician who will be doing the installation work next week is included. She should be in touch soon, but please also feel free to give him a call if you want to accompany him on any visits or would like to speak with him about anything else.

Thanks again for all of your assistance and the quick turnaround!

We will be in touch with you again once the visits scheduled below are complete.

<Insert schedule from excel spreadsheet, redact addresses/phone numbers $>$ 


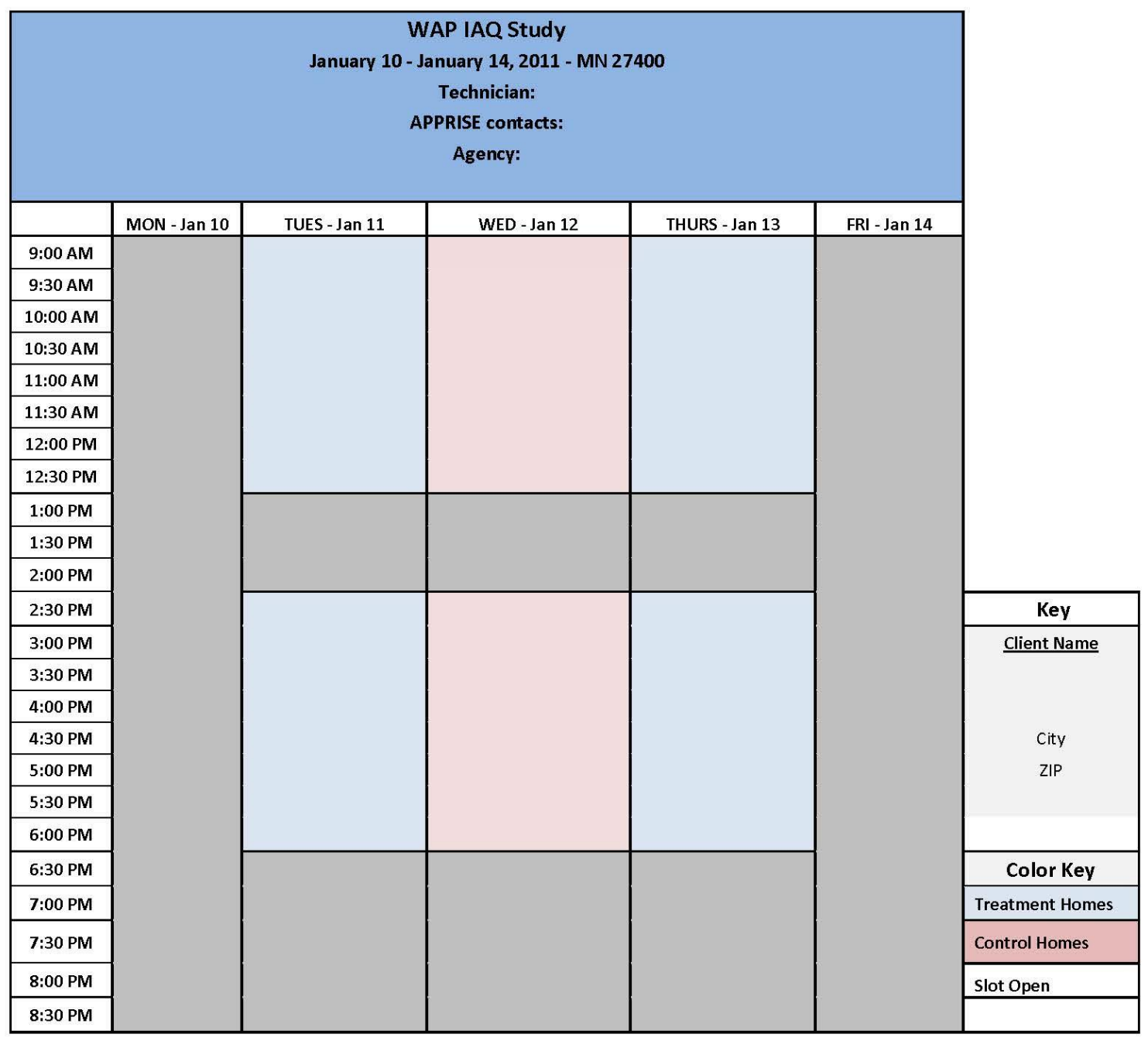


Subject: WAP IAQ study post monitoring-installation, pre-wx update

Dear,

This is a brief update on your agency's homes participating in the national WAP evaluation indoor air quality field study. All the pre-weatherization tech visits to homes are now complete. Our tech made the first visits according to the schedule we previously emailed you (below). During the week of Jan 17 second visits were made to each home to pick up some of the sample canisters (radon, formaldehyde, etc.).

The next step will be for you to weatherize these homes during the "weatherization window." Please see the table below for a list of participating homes and their weatherization windows.

\begin{tabular}{|c|c|}
\hline $\begin{array}{c}\text { Treatment Group: } \\
\text { To be weatherized between Jan } \mathbf{3 1} \text { and Feb 22 }\end{array}$ & $\begin{array}{c}\text { Control Group: } \\
\text { To be weatherized after Mar 21 }\end{array}$ \\
\hline & \\
\hline & \\
\hline & \\
\hline & \\
\hline & \\
\hline
\end{tabular}

A couple of very important reminders:

- It is critical for this study that weatherization fit into these windows. If anything comes up that affects or delays this plan (e.g. emergency furnace replacements), please let me know.

Weatherization work on treatment homes should not be initiated until January 31, and should be completed by February 22.

Please be aware that these homes have been outfitted with monitoring equipment that may include: carbon monoxide monitors, temperature/humidity monitors and thermocoupling loggers on unvented combustion appliances. It is important that is equipment NOT be disturbed during the weatherization process.

Weatherization work on control homes should not be initiated until March 21.

- Please be sure to let all relevant scheduling staff and your crews know about these special circumstances.

All the other homes on your candidate list can now be weatherized as usual - they are not participating in the study.

Note that I have $\mathrm{cc}^{\prime} \mathrm{d}$ name, your Case Manager for other national WAP evaluation matters. Name may also be sending indoor air quality field study updates in the future.

- Please reply and confirm your receipt of this note. 
Thanks again for your assistance with the study. If you have any questions please let me know. We'll be in touch soon to see how weatherization is going on the Treatment homes.

H-11 
Subject: WAP IAQ treatment home weatherization check-in

Dear

I am emailing to check in with you and see how weatherization is going on your agency's homes participating in the national WAP evaluation indoor air quality study - specifically the treatment homes.

As discussed earlier, all \# treatment homes should be weatherized between Date and Date.

The treatment homes to be weatherized during this window are listed below.

\begin{tabular}{|c|}
\hline Treatment Group: \\
To be weatherized between \\
\hline \\
\hline \\
\hline \\
\hline \\
\hline
\end{tabular}

- Please be aware that these homes have been outfitted with monitoring equipment that may include: carbon monoxide monitors, temperature/humidity monitors and thermo-coupling loggers on unvented combustion appliances. It is important that is equipment NOT be disturbed during the weatherization process.

- Please contact me if there is anything you feel we should know about that might affect the study, or if there is anything that leads you to believe the homes will not be completely weatherized during the study time-frame.

- Please do not weatherize control homes until after DATE.

We will contact you at the end of this weatherization period to verify that weatherization has been completed on your treatment homes.

Thank you!

<cc Nick> 
Subject: WAP IAQ treatment home weatherization confirmation

Dear,

I am emailing to confirm that the weatherization of your \# treatment homes participating in the WAP evaluation indoor air quality study is complete.

As discussed earlier, all \# treatment homes should have been weatherized between Jan 31 and yesterday Feb 22.

The treatment homes that should have been weatherized during this window are listed below.

\begin{tabular}{|c|}
\hline Treatment Group: \\
\hline Weatherized between Jan 31 and Feb 22 \\
\hline \\
\hline \\
\hline \\
\hline \\
\hline
\end{tabular}

Please note that we will contact all study participants in the near future to schedule our next technician monitoring visit (relevant dates below).

\section{Key dates}

- First round of monitoring visits (3-4 hours per home) -- COMPLETED

- Monitoring visit \#2 - pickup of 7-day monitors ( 1 hour per home) -- COMPLETED

- Weatherization window for treatment homes -- COMPLETED?

- Second round of monitoring visits (3-4 hours per home) -- Mar 7-Mar 11

- Monitoring visit \#4 - pickup visits for 7-day monitors ( 1 hour per home) -- Mar 14 - Mar 18

- Mar 21 -- Weatherization can begin for control homes

Please respond to this email verifying that weatherization is complete on your treatment homes. If you haven't had a chance to reply soon, I'll follow up with a phone call.

Thank you for working with the study time frames.

$<\mathrm{CC}$ case manager; Nick $>$ 
Subject: WAP IAQ study - schedule for second round of monitoring visits

Dear,

We have scheduled your \# study homes for the first visit of the second round of WAP evaluation indoor air quality study monitoring. The schedule for these technician visits is below, and uses the same format we used during the first round.

As before, the name and phone number of the technician who will be doing the installation work next week is included. Please contact him if you wish to accompany him on any visits or if you have questions or concerns.

\section{Monitoring visits}

As in the first round of visits, our technician will be in your area the week of Mar 7 to perform basic diagnostic tests and install monitoring equipment on all \# homes. Each visit will take about $3+$ hours.

\section{Key dates}

- First round of monitoring visits (3-4 hours per home) -- COMPLETED

- Pickup visits for 7-day monitors ( 1 hour per home) -- COMPLETED

- Weatherization window for treatment homes - COMPLETED

- Mar 7 - Mar 11: second round of monitoring visits (3-4 hours per home)

- Mar 14-Mar 18: pickup visits for 7-day monitors ( 1 hour per home)

- Mar 21 - weatherization can begin for control homes

We will be in touch with you again once the visits scheduled below are complete.

$<<$ Please note: We have been unable to reach on the phone and were forced to mail $\mathrm{him} /$ her a letter to schedule this appointment. If you could reach out to as soon as possible to inform him/her of the appointment and ask him to contact us, we would be very grateful.>>

<Insert schedule from excel spreadsheet, redact addresses/phone numbers $>$

Thanks again for all of your assistance! Regards,

$<c c$ case manager; Nick > 
Subject: WAP IAQ study fieldwork complete

Dear

We are delighted to inform you that with your help we will be completing the field study portion of the WAP evaluation indoor air quality study in the next few days.

The $(\#)$ control homes participating in the study may be weatherized beginning next week. Those homes are listed below.

The control homes to be weatherized

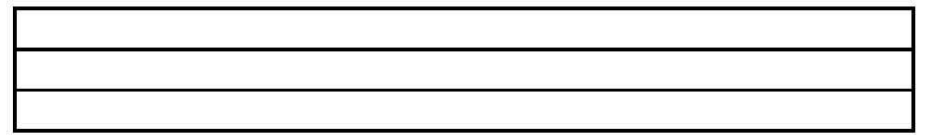

On behalf of the entire WAP evaluation team, I want to thank you for your active participation in the study. Your work was essential to the effort.

Sincerely,

$<$ CC Nick $>$ 

APPENDIX I. HOUSEHOLD RECRUITMENT SCHEDULING SCRIPTS AND INFORMATION FOR STUDY PARTICIPANTS 



\title{
APPENDIX I. HOUSEHOLD RECRUITMENT SCHEDULING SCRIPTS AND INFORMATION FOR STUDY PARTICIPANTS
}

\author{
IAQ Recruitment Script
}

INTRO:

Hi, may I speak to $?$

Good morning/afternoon/evening, my name is I'm calling from APPRISE on behalf of the national Weatherization Assistance Program Evaluation. You will be receiving weatherization services from [STATE WAP PROGRAM] through [AGENCY NAME].

We are contacting weatherization clients who may qualify to participate in a national research study. This study will help us to get a better understanding of the impacts of the weatherization program on the air quality in your home. For this study, a technician will visit your home to conduct some tests and install some equipment in your home.

In total, we will make four visits to your home, and you will receive $<\$ 120$ (if treatment) or $\$ 170$ (if control) $>$ in appreciation for your time. The first visit will be next week when a technician will conduct tests and install equipment in your home. This visit will take about 4 hours and you will receive the first $\$ 50$, as a check. One week later, an interviewer will come to your home to pick up certain equipment and ask you to complete a telephone survey then. This second visit will take about 1 hour and you will receive the next $\$ 10$.

[FOR NOV 1 HHOLDS]: Your third visit will be on the week of January 17, 2011. [FOR NOV 8 HHOLDS]: Your third visit will be on the week of January 24, 2011. [FOR NOV 29 HHOLDS]: Your third visit will be on the week of February 7, 2011. [FOR DEC 6 HHOLDS]: Your third visit will be on the week of February 14, 2011. [FOR JAN 3 HHOLDS]: Your third visit will be on the week of February 28, 2011. [FOR JAN 10 HHOLDS]: Your third visit will be on the week of March 7, 2011.

[FOR ALL]: Your fourth and final visit will take place one week after the third visit. You will receive $<\$ 50$ (if treatment) or $\$ 100$ (if control) $>$ on the third visit and the final $\$ 10$ on the fourth visit.

TREATMENT HOMES:

[STATE WAP PROGRAM] will weatherize your home in-between these sets of visits. For your home, that would be...

[FOR NOV 1 HHOLDS]: between Nov 22 and Dec 31.

[FOR NOV 8 HHOLDS]: between Nov 29 and Jan 7.

[FOR NOV29 HHOLDS]: between Dec 20 and Jan 21.

[FOR DEC 6 HHOLDS]: $\quad$ between Dec 27 and Jan 28.

[FOR JAN 3 HHOLDS]: between Jan 24 and Feb 15.

[FOR JAN 10 HHOLDS]: between Jan 31 and Feb 22. 
CONTROL HOMES:

[STATE WAP PROGRAM] will weatherize your home after these visits. For your home, that would be...

[FOR NOV 1 HHOLDS]: sometime after Jan 31.

[FOR NOV 8 HHOLDS]: sometime after Feb 7.

[FOR NOV29 HHOLDS]: sometime after Feb 21.

[FOR DEC 6 HHOLDS]: $\quad$ sometime after Feb 28.

[FOR JAN 3 HHOLDS]: $\quad$ sometime after Mar 14.

[FOR JAN 10 HHOLDS]: sometime after Mar 21.

[Optional Talking Points for Those with Concerns]

What it involves:

- The first visit should take about 4 hours, but you'11 receive a $\$ 50$ check at the time of the visit.

- The second visit will occur one week later, when an interviewer comes to collect the equipment. The visit should take about 1 hour, and you'1l receive another $\$ 10$ at the time of the visit. You will be asked to dial a toll-free number to complete a phone survey.

- The third visit will be similar to the first visit, when equipment is installed.

- The fourth visit will be another pick up visit.

- Please be aware that weatherization services will not be provided during the visit.

Legitimacy concerns:

[AGENCY] is currently contacting weatherization clients who may qualify for this study to let you know that we would be calling you. If you haven't heard from them yet, I can give you the contact information of someone at [AGENCY] if you would like.

- Contact:

- The technician who will be visiting you is $\mathrm{He} / \mathrm{she}$ will be wearing a WAP evaluation name tag with the WAP logo on it. [IF APPLICABLE: will be coming with him/her to monitor his/her work and for quality control purposes.]

- You will receive a letter of confirmation from APPRISE. The letter will confirm the name of the technician who will conduct the visit and include our contact information in case something comes up and you need to reschedule the visit.

- APPRISE will also call you the day before the visit to remind you of the visit.

- APPRISE website http://www.appriseinc.org

- APPRISE is a nonprofit research company. We're in partner with ECW and responsible for calling respondents and scheduling appointments for this national weatherization study.

Privacy concerns:

Air quality data for your home will be anonymously pooled with other homes around the country to assess the impacts of the weatherization program on the air quality in your home. Your data will not be reported individually for the national evaluation. Participation in this research will not affect your eligibility to participate in [STATE WAP NAME] or any other energy assistance program. We may provide information on your air quality to [AGENCY], which may see individual household data on air quality before and after weatherization. 
SCREENER:

Great, before we schedule, I just have a few questions for you to make sure that you qualify for the study.

Q1) Is your home a...?
1) single-family home
$=>$ CONTINUE
2) row or townhouse
$=\Rightarrow$ CONTINUE
3) a unit in a multi-family structure $=\Rightarrow$ DOES NOT QUALIFY; SKIP TO END
4) a mobile home or house trailer $\Rightarrow$ CONTINUE
5) something else
$=\Rightarrow$ DOES NOT QUALIFY; SKIP TO END
8) DON'T KNOW
$==>$ DOES NOT QUALIFY; SKIP TO END
9) REFUSED
$==$ DOES NOT QUALIFY; SKIP TO END

Q2) Do you plan to move out of this home before next March?
1) no
$==>$ CONTINUE
2) yes
$==>$ DOES NOT QUALIFY; SKIP TO END
8) DON'T KNOW
$==>$ DOES NOT QUALIFY; SKIP TO END
9) REFUSED
$=\Rightarrow$ DOES NOT QUALIFY; SKIP TO END

Q3) Do you expect that your home will be vacant for more than a total of three weeks between this November and next March?
1) no
$=>$ CONTINUE
2) yes
$\Rightarrow$ DOES NOT QUALIFY; SKIP TO END
8) DON'T KNOW
$=\Rightarrow$ DOES NOT QUALIFY; SKIP TO END
9) REFUSED
$==>$ DOES NOT QUALIFY; SKIP TO END

Q4) What is the main heating fuel that you use to heat your home? READ IF NECESSARY
1) ELECTRICITY
2) NATURAL GAS FROM UNDERGROUND PIPES
3) PROPANE (BOTTLED GAS)
4) FUEL OIL
5) KEROSENE
6) WOOD
7) SOME OTHER FUEL:

Q5) What is your main heating equipment? READ IF NECESSARY
1) HEAT PUMP
2) CENTRAL FURNACE WITH DUCTS TO INDIVIDUAL ROOMS
3) STEAM/HOT WATER SYSTEM WITH RADIATORS OR PIPES IN EACH ROOM
4) BUILT-IN ELECTRIC UNITS IN EACH ROOM INSTALLED IN WALLS, CEILINGS, BASEBOARDS, OR FLOORS
5) BUILT-IN FLOOR/WALL PIPELESS FURNACE
6) BUILT-IN ROOM HEATER BURNING GAS, OIL, OR KEROSENE
7) HEATING STOVE BURNING WOOD, COAL, OR COKE
8) PORTABLE ELECTRIC HEATERS 
9) PORTABLE KEROSENE HEATERS

10) FIREPLACE

11) SOME OTHER EQUIPMENT:

Q6) And is that heating equipment functional?

1. NO

2. YES

Q7) What other heating equipment do you use in your home? CHECK ALL THAT APPLY none

1. HEAT PUMP

2. CENTRAL FURNACE WITH DUCTS TO INDIVIDUAL ROOMS

3. STEAM/HOT WATER SYSTEM WITH RADIATORS OR PIPES IN EACH ROOM

4. BUILT-IN ELECTRIC UNITS IN EACH ROOM INSTALLED IN WALLS, CEILINGS, BASEBOARDS, OR FLOORS

5. BUILT-IN FLOOR/WALL PIPELESS FURNACE

6. BUILT-IN ROOM HEATER BURNING GAS, OIL, OR KEROSENE

7. HEATING STOVE BURNING WOOD, COAL, OR COKE

8. PORTABLE ELECTRIC HEATERS

9. PORTABLE KEROSENE HEATERS

10. FIREPLACE

11. SOME OTHER EQUIPMENT :

Q8) Is your oven...?

1) Electric

2) Natural gas or propane

3) Other:

4) Don't have an oven

Q9) Which of the following best describes how much you use your oven?

1) Never

2) Rarely

3) ---Sometimes

4) Frequently

5) All the time

8) DON'T KNOW

9) REFUSED 
QUALIFIED:

Great, you qualify for this study. When would be a good time for the technician to come to your home for the first visit?

[NOTE: If the respondent is interested but none of the times work, ask them what would be a good time, and if stated times are doable for the field technician (weekday day \& evening, Saturday morning), then say: I'm sorry, but none of the times you told me work because we're already booked then. However, I can put you down as interested, and if someone cancels for any reason, someone from APPRISE or the field technician can call you back to try to schedule you in? If yes, continue with screener.]

Just to make sure, we agreed that your appointment will be on:

Now I would also like to schedule the second visit pick up, which occurs one week after the first visit, so that the equipment can be collected and you can complete a phone survey. The visit will last one hour.

Since your appointment is on the pick up date will be scheduled [SEE TABLE]

\begin{tabular}{|l|l|}
\hline Instrumentation & Pick up date* \\
\hline Tues pm & Next Wed pm \\
\hline Wed am & Next Wed pm \\
\hline Wed pm & Next Thurs am \\
\hline Thurs am & Next Thurs am \\
\hline Thurs pm & Next Thurs pm \\
\hline Friday am & Next Thurs pm \\
\hline Friday pm & Next Friday am \\
\hline
\end{tabular}

* Can be moved to the prior or next box, if necessary.

[INTERVIEWER NOTE: the pick up date is time sensitive. If the respondent cannot make the pick up date, reschedule the appointment date.]

\section{CHECK NAME SPELLING AND ADDRESS!}

Do you have another phone number you can give us in case we can't reach you at this one? VERIFY THIS NUMBER AND THE ALTERNATE NUMBER!

If you need to reschedule, please call APPRISE toll free at 1-888-434-8008 and mention the WAP study. During the visit, please make sure that the technician will have access to ALL rooms, and as a courtesy, if you have any pets, please try to keep them out of the way while the technician is conducting the tests.

\section{NOT QUALIFIED:}

I'm sorry. You're not eligible to participate in this study at this time. However, if we don't find enough qualified respondents, we may change the qualification criteria and we might call you back. Thank you very much for your time and have a great day. 


\section{IAQ Recruitment Script}

\section{INTRO:}

Hi, may I speak to $?$

Good morning/afternoon/evening, my name is behalf of the national Weatherization Assistance Program Evaluation.

You recently received a visit from a technician and pick-up interviewer. I'm calling to schedule your third visit. Next week a technician will conduct tests and install equipment in your home. This visit will take about 4 hours and you will receive a check for $<\$ 50$ (if treatment) or $\$ 100$ (if control) $>$ One week later, an interviewer will come to your home to pick up certain equipment and ask you to complete a telephone survey then. This second visit will take about 1 hour and you will receive the next $\$ 10$.

[FOR NOV 1 HHOLDS]: Your third visit will be on the week of January 17, 2011.

[FOR NOV 8 HHOLDS]: Your third visit will be on the week of January 24, 2011.

[FOR NOV 29 HHOLDS]: Your third visit will be on the week of February 7, 2011.

[FOR DEC 6 HHOLDS]: Your third visit will be on the week of February 14, 2011.

[FOR JAN 3 HHOLDS]: Your third visit will be on the week of February 28, 2011.

[FOR JAN 10 HHOLDS]: Your third visit will be on the week of March 7, 2011.

[Optional Talking Points for Those with Concerns]

TREATMENT HOMES:

[STATE WAP PROGRAM] will weatherize your home in-between these sets of visits. For your home, that would be...

[FOR NOV 1 HHOLDS]: between Nov 22 and Dec 31.

[FOR NOV 8 HHOLDS]: between Nov 29 and Jan 7.

[FOR NOV29 HHOLDS]: between Dec 20 and Jan 21.

[FOR DEC 6 HHOLDS]: between Dec 27 and Jan 28.

[FOR JAN 3 HHOLDS]: between Jan 24 and Feb 15.

[FOR JAN 10 HHOLDS]: between Jan 31 and Feb 22.

CONTROL HOMES:

[STATE WAP PROGRAM] will weatherize your home after these visits. For your home, that would be...

[FOR NOV 1 HHOLDS]: sometime after Jan 31.

[FOR NOV 8 HHOLDS]: sometime after Feb 7.

[FOR NOV29 HHOLDS]: sometime after Feb 21.

[FOR DEC 6 HHOLDS]: sometime after Feb 28.

[FOR JAN 3 HHOLDS]: sometime after Mar 14.

[FOR JAN 10 HHOLDS]: sometime after Mar 21. 
- Please be aware that weatherization services will not be provided during the visit.

Legitimacy concerns:

[AGENCY] is currently contacting weatherization clients who may qualify for this study to let you know that we would be calling you. If you haven't heard from them yet, I can give you the contact information of someone at [AGENCY] if you would like.

- Contact:

- The technician who will be visiting you is $\mathrm{He} /$ she will be wearing a WAP evaluation name tag with the WAP logo on it. [IF APPLICABLE: will be coming with him/her to monitor his/her work and for quality control purposes.]

- You will receive a letter of confirmation from APPRISE. The letter will confirm the name of the technician who will conduct the visit and include our contact information in case something comes up and you need to reschedule the visit.

- APPRISE will also call you the day before the visit to remind you of the visit.

- APPRISE website http://www.appriseinc.org

- APPRISE is a nonprofit research company. We're in partner with $\mathrm{ECW}$ and responsible for calling respondents and scheduling appointments for this national weatherization study.

Privacy concerns:

Air quality data for your home will be anonymously pooled with other homes around the country to assess the impacts of the weatherization program on the air quality in your home. Your data will not be reported individually for the national evaluation. Participation in this research will not affect your eligibility to participate in [STATE WAP NAME] or any other energy assistance program. We may provide information on your air quality to [AGENCY], which may see individual household data on air quality before and after weatherization.

\section{CHECK NAME SPELLING AND ADDRESS!}

Do you have another phone number you can give us in case we can't reach you at this one? VERIFY THIS NUMBER AND THE ALTERNATE NUMBER!

If you need to reschedule, please call APPRISE toll free at 1-888-434-8008 and mention the WAP study. During the visit, please make sure that the technician will have access to ALL rooms, and as a courtesy, if you have any pets, please try to keep them out of the way while the technician is conducting the tests. 


\section{IAQ Recruitment Script}

\section{INTRO:}

Hi, may I speak to $?$

Good morning/afternoon/evening, my name is behalf of the national Weatherization Assistance Program Evaluation.

You recently received a visit from a technician and pick-up interviewer. I'm calling to schedule your third visit. During the week of a technician will conduct tests and install equipment in your home. This visit will take about 4 hours and you will receive a check for $<\mathbf{\$ 5 0}$ (if treatment) or $\$ \mathbf{1 0 0}$ (if control)> One week later, an interviewer will come to your home to pick up certain equipment. This second visit will take about $1 / 2$ hour and you will receive the next $\$ 10$.

\section{- Please be aware that weatherization services will not be provided during the visit.}

\section{Legitimacy concerns:}

[AGENCY] is currently contacting weatherization clients who may qualify for this study to let you know that we would be calling you. If you haven't heard from them yet, I can give you the contact information of someone at [AGENCY] if you would like.

- Contact:

- The technician who will be visiting you is He/she will be wearing a WAP evaluation name tag with the WAP logo on it. [IF APPLICABLE: will be coming with him/her to monitor his/her work and for quality control purposes.]

- You will receive a letter of confirmation from APPRISE. The letter will confirm the name of the technician who will conduct the visit and include our contact information in case something comes up and you need to reschedule the visit.

- APPRISE will also call you the day before the visit to remind you of the visit.

- APPRISE website http://www.appriseinc.org

- APPRISE is a nonprofit research company. We're in partner with $\mathrm{ECW}$ and responsible for calling respondents and scheduling appointments for this national weatherization study.

\section{Privacy concerns:}

Air quality data for your home will be anonymously pooled with other homes around the country to assess the impacts of the weatherization program on the air quality in your home. Your data will not be reported individually for the national evaluation. Participation in this research will not affect your eligibility to participate in [STATE WAP NAME] or any other energy assistance program. We may provide information on your air quality to [AGENCY], which may see individual household data on air quality before and after weatherization. 


\section{CHECK NAME SPELLING AND ADDRESS!}

Do you have another phone number you can give us in case we can't reach you at this one? VERIFY THIS NUMBER AND THE ALTERNATE NUMBER!

If you need to reschedule, please call APPRISE toll free at 1-888-434-8008 and mention the WAP study. During the visit, please make sure that the technician will have access to ALL rooms, and as a courtesy, if you have any pets, please try to keep them out of the way while the technician is conducting the tests. 


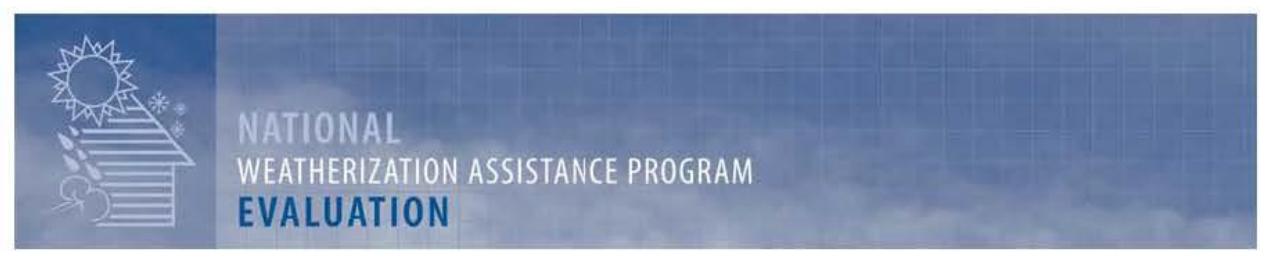

\section{Information for Occupants Indoor Air Quality Study}

We appreciate your willingness to participate in a study on the effectiveness of home weatherization. This work is in addition to the weatherization to be performed on your home, and is being done by a separate research team in cooperation with your local weatherization agency.

We'll be doing some testing of your home, similar to some of the tests run by your weatherization agency. In addition, we'll be installing some measurement equipment, including

- A temperature and humidity recording device at or near your thermostat

- A device to record carbon monoxide levels in your home

- A meter to record electricity use by your refrigerator

- In some cases, a device to record the operation of certain heating or gas burning appliances

- One or more samplers to test for radon

- Possibly, one or more samplers to test for other indoor air quality indicators

Please do not disturb any of the devices left in your home. If any of these items are disturbed or removed, please call us at the number below. Please keep the windows in your home closed for the next week (until the sampling devices are picked up) to ensure accurate test results.

We will ask to return to your home 3 more times. One visit will be similar to the one today, including some testing of your home. The other two visits will be very brief, to stop by and pick up some of the sampling devices. One of our team members will be calling you in a few days, to schedule a time next week to stop by.

Carbon monoxide is a poisonous gas, and the recording device placed in your home will sound an alarm if carbon monoxide exceeds a certain level. If you hear an alarm from this device, you should immediately consider what the source of carbon monoxide might be, and stop its operation. Possible sources include any unvented gas or oil heating device, gas oven, a car or truck running in an attached garage, devices with engines (generator sets), grills, and smoking. In some cases, fireplaces, wood stoves, furnaces or water heaters may operate under conditions that cause combustion products including carbon monoxide to leak back into the home.

If you need to contact us about this project, use the phone number below. If you have questions about the weatherization work being done in your home, please call your local weatherization agency.

Thank you

National Weatherization Evaluation Team

Energy Center of Wisconsin

608-238-8276 


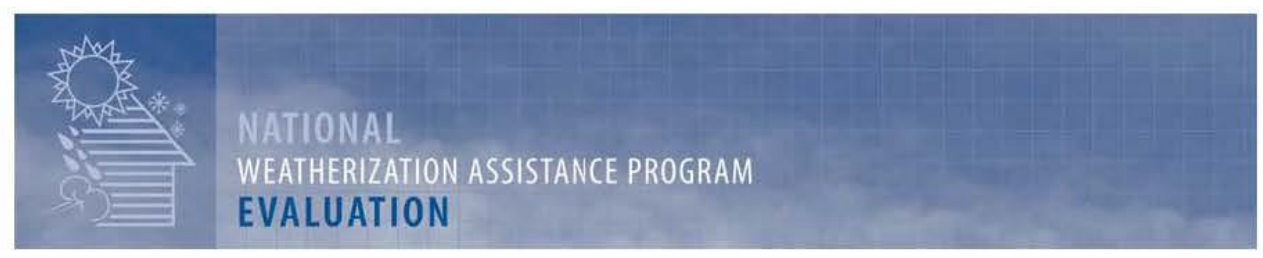

\section{Notification of possible hazardous conditions}

While testing your home as part of our research project, we have identified conditions that may be hazardous. Our work is not intended to provide a complete evaluation of hazards related to combustion systems, fuel use, or any other type of hazards.

\section{Carbon Monoxide}

a) One or more vented combustion appliances in your home (indicated below) produces carbon monoxide $(\mathrm{CO})$ at a level greater than 100 parts per million in the vent system, which may be a health hazard if it escapes into the home. We recommend further investigation and possible repair of this appliance.

b) One or more vented combustion appliances in your home (indicated below) produces carbon monoxide $(\mathrm{CO})$ at a level greater than 100 parts per million combined with spillage from the vent system of longer than one minute under normal operating conditions. This may be a health hazard. We recommend against using the appliance until further diagnosed and repaired.

c) One or more vented combustion appliances in your home (indicated below) produces carbon monoxide (CO) at a level greater than 400 parts per million in the vent system. This may be a health hazard if it escapes into the home. We recommend against using the appliance until further diagnosed and repaired.

\section{Furnace}

Boiler

Other

Water Heater
CO concentration at vent, parts per million $\mathrm{CO}$ concentration at vent, parts per million $\mathrm{CO}$ concentration at vent, parts per million $\mathrm{CO}$ concentration at vent, parts per million

d) One or more unvented gas appliances in your home (indicated below) produces carbon monoxide at a level greater than $100 \mathrm{ppm}$ in the appliance vent ( $800 \mathrm{ppm}$ "air-free" in the case of ovens), or 50 parts per million in air near the appliance. We recommend further investigation and possible repair of this appliance.

e) The carbon monoxide level in an area in which we were testing reached $35 \mathrm{ppm}$ (parts per million). This indicates probable carbon monoxide production and spillage from one or more appliances, as indicated below. We recommend further investigation and repair of this appliance. Under our rules, we cannot continue with our testing of this appliance. 


\section{Combustible gas leakage}

a) We have identified one or more minor gas leaks in your home. While these leaks do not appear to be large, we recommend they be repaired. We found leaks at the following locations:

b) We have identified one or more concentrated gas leaks in your home. These leaks may be hazardous. We strongly recommend that the appliances near these leaks not be operated and that the leaks be immediately repaired by a qualified gas service technician. We found leaks at the following locations:

We recommend the entire gas system be checked when you have any service done.

\section{Acknowledgement of receipt of Notification of possible hazards (by adult occupant)}

Name

Address

Signature Date

Directions for technician:

1) Circle entries that apply, cross out entries that do not apply

2) Fill in measurement quantities and locations for all entries that do apply

3) Complete 2 copies, one for occupants, one for project team

4) An adult (18 or older) permanent occupant should sign both copies

5) If concentrated gas leaks identified, call Tech Hotine 877-297-2797

6) If other serious immediate hazard is identified, callemergency services if warranted, and call Tech Hotine immediately 877-297-2797 


\title{
Weatherization Assistance Program Evaluation Team
}

Receipt for Incentive Payment

\author{
Name \\ Full Address \\ Check or gift card number \\ Value of check or gift card
}

I received a check or gift card in the value shown above

Signature

Date

Thank You!

\section{Form prepared by}

Energy Center of Wisconsin

October, 2010 

APPENDIX J. FIELD DEPLOYMENT SCHEDULE 



\section{APPENDIX J. FIELD DEPLOYMENT SCHEDULE}

Table J.1 Field Deployment Schedule.

\begin{tabular}{|c|c|c|c|c|c|c|c|c|}
\hline \multirow{2}{*}{ Round } & \multirow{2}{*}{ State } & \multirow{2}{*}{ SuperPUMA } & \multirow{2}{*}{$\begin{array}{c}\text { Pre-Wx } \\
\text { Testing week }\end{array}$} & \multicolumn{2}{|c|}{ Weatherization window } & \multirow{2}{*}{$\begin{array}{c}\text { Post-Wx } \\
\text { Testing week } \\
\end{array}$} & \multicolumn{2}{|c|}{ Sites } \\
\hline & & & & start & end & & control & treatment \\
\hline 1 & $\mathrm{IL}$ & 17030 & 1-Nov-10 & 22-Nov-10 & 31-Dec-10 & 31-Jan-11 & 2 & 5 \\
\hline 1 & $\mathrm{IL}$ & 17070 & 1-Nov-10 & 22-Nov-10 & 31-Dec-10 & 24-Jan-11 & 3 & 5 \\
\hline 1 & IA & 19100 & 1-Nov-10 & 22-Nov-10 & 31-Dec-10 & 17-Jan-11 & 3 & 5 \\
\hline 1 & MA & 25070 & 1-Nov-10 & 22-Nov-10 & 31-Dec-10 & 17-Jan-11 & 3 & 5 \\
\hline 1 & $\mathrm{NE}$ & 31201 & 1-Nov-10 & 22-Nov-10 & 31-Dec-10 & 17-Jan-11 & 3 & 5 \\
\hline 1 & $\mathrm{ND}$ & 38100 & 1-Nov-10 & 22-Nov-10 & 31-Dec-10 & 17-Jan-11 & 3 & 4 \\
\hline 1 & $\mathrm{WI}$ & 55900 & 1-Nov-10 & 22-Nov-10 & 31-Dec-10 & 24-Jan-11 & 3 & 5 \\
\hline 2 & $\mathrm{CT}$ & 9600 & 8-Nov-10 & 29-Nov-10 & 7-Jan-11 & 17-Jan-11 & 3 & 5 \\
\hline 2 & ID & 16200 & 8-Nov-10 & 29-Nov-10 & 7-Jan-11 & 31-Jan-11 & 3 & 4 \\
\hline 2 & $\mathrm{IL}$ & 17020 & 8-Nov-10 & 29-Nov-10 & 7-Jan-11 & 24-Jan-11 & 4 & 3 \\
\hline 2 & IA & 19300 & 8-Nov-10 & 29-Nov-10 & 7-Jan-11 & 24-Jan-11 & 3 & 3 \\
\hline 2 & IA & 19400 & 8-Nov-10 & 29-Nov-10 & 7-Jan-11 & 31-Jan-11 & 2 & 6 \\
\hline 2 & WI & 55100 & 8-Nov-10 & 29-Nov-10 & 7-Jan-11 & 24-Jan-11 & 3 & 5 \\
\hline 2 & WI & 55400 & 8-Nov-10 & 29-Nov-10 & 7-Jan-11 & 24-Jan-11 & 3 & 5 \\
\hline 3 & $\mathrm{CO}$ & 8103 & 29-Nov-10 & 20-Dec-10 & 21-Jan-11 & 7-Feb-11 & 3 & 5 \\
\hline 3 & IN & 18060 & 29-Nov-10 & 20-Dec-10 & 21-Jan-11 & 21-Feb-11 & 4 & 3 \\
\hline 3 & IA & 19200 & 29-Nov-10 & 20-Dec-10 & 21-Jan-11 & 7-Feb-11 & 2 & 5 \\
\hline 3 & KY & 21500 & 29-Nov-10 & 20-Dec-10 & 21-Jan-11 & 7-Feb-11 & 3 & 5 \\
\hline 3 & MI & 26070 & 29-Nov-10 & 20-Dec-10 & 21-Jan-11 & 7-Feb-11 & 3 & 5 \\
\hline 3 & $\mathrm{MO}$ & 29500 & 29-Nov-10 & 20-Dec-10 & 21-Jan-11 & 7-Feb-11 & 2 & 6 \\
\hline 3 & $\mathrm{NC}$ & 37060 & 29-Nov-10 & 20-Dec-10 & 21-Jan-11 & 7-Feb-11 & 3 & 5 \\
\hline 3 & SD & 46100 & 29-Nov-10 & 20-Dec-10 & 21-Jan-11 & 7-Feb-11 & 3 & 5 \\
\hline 3 & UT & 49100 & 29-Nov-10 & 20-Dec-10 & 21-Jan-11 & 7-Feb-11 & 3 & 5 \\
\hline 3 & WA & 53050 & 29-Nov-10 & 20-Dec-10 & 21-Jan-11 & 7-Feb-11 & 3 & 5 \\
\hline 3 & WV & 54100 & 29-Nov-10 & 20-Dec-10 & 21-Jan-11 & 7-Feb-11 & 2 & 6 \\
\hline 4 & $\mathrm{CA}$ & 6090 & 6-Dec-10 & 27-Dec-10 & 28-Jan-11 & 14-Feb-11 & 3 & 5 \\
\hline 4 & $\mathrm{CA}$ & 6411 & 6-Dec-10 & 27-Dec-10 & 28-Jan-11 & 14-Feb-11 & 3 & 4 \\
\hline 4 & $\mathrm{CO}$ & 8203 & 6-Dec-10 & 27-Dec-10 & 28-Jan-11 & 14-Feb-11 & 2 & 4 \\
\hline 4 & IN & 18080 & 6-Dec-10 & 27-Dec-10 & 28-Jan-11 & 21-Feb-11 & 3 & 5 \\
\hline 4 & $\mathrm{KS}$ & 20200 & 6-Dec-10 & 27-Dec-10 & 28-Jan-11 & 14-Feb-11 & 3 & 4 \\
\hline 4 & $\mathrm{KY}$ & 21300 & 6-Dec-10 & 27-Dec-10 & 28-Jan-11 & 14-Feb-11 & 3 & 5 \\
\hline 4 & $\mathrm{MD}$ & 24300 & 6-Dec-10 & 27-Dec-10 & 28-Jan-11 & 14-Feb-11 & 3 & 3 \\
\hline 4 & MI & 26132 & 6-Dec-10 & 27-Dec-10 & 28-Jan-11 & 28-Feb-11 & 2 & 4 \\
\hline 4 & $\mathrm{MO}$ & 29300 & 6-Dec-10 & 27-Dec-10 & 28-Jan-11 & 14-Feb-11 & 3 & 5 \\
\hline 4 & $\mathrm{NC}$ & 37010 & 6-Dec-10 & 27-Dec-10 & 28-Jan-11 & 14-Feb-11 & 3 & 5 \\
\hline 4 & $\mathrm{VA}$ & 51120 & 6-Dec-10 & 27-Dec-10 & 28-Jan-11 & 21-Feb-11 & 3 & 5 \\
\hline 4 & WA & 53100 & 6-Dec-10 & 27-Dec-10 & 28-Jan-11 & 14-Feb-11 & 1 & 7 \\
\hline 5 & ID & 16100 & 3-Jan-11 & 24-Jan-11 & $15-F e b-11$ & 28-Feb-11 & 2 & 4 \\
\hline 5 & $\mathrm{ME}$ & 23200 & 3-Jan-11 & 24-Jan-11 & $15-F e b-11$ & 28-Feb-11 & 2 & 4 \\
\hline 5 & MT & 30100 & 3-Jan-11 & 24-Jan-11 & 15-Feb-11 & 28-Feb-11 & 1 & 2 \\
\hline 5 & $\mathrm{NJ}$ & 34090 & 3-Jan-11 & 24-Jan-11 & 15-Feb-11 & 28-Feb-11 & 2 & 3 \\
\hline 5 & NM & 35300 & 3-Jan-11 & 24-Jan-11 & 15-Feb-11 & 28-Feb-11 & 2 & 3 \\
\hline
\end{tabular}


Table J.1 Field Deployment Schedule.

\begin{tabular}{|c|c|c|c|c|c|c|c|c|}
\hline \multirow{2}{*}{ Round } & \multirow{2}{*}{ State } & \multirow{2}{*}{ SuperPUMA } & \multirow{2}{*}{$\begin{array}{c}\text { Pre-Wx } \\
\text { Testing week }\end{array}$} & \multicolumn{2}{|c|}{ Weatherization window } & \multirow{2}{*}{$\begin{array}{c}\text { Post-Wx } \\
\text { Testing week } \\
\end{array}$} & \multicolumn{2}{|c|}{ Sites } \\
\hline & & & & start & end & & control & treatment \\
\hline 5 & NY & 36021 & 3-Jan-11 & 24-Jan-11 & 15-Feb-11 & 28-Feb-11 & 3 & 4 \\
\hline 5 & NY & 36081 & 3-Jan-11 & 24-Jan-11 & 15-Feb-11 & 28-Feb-11 & 2 & 4 \\
\hline 5 & $\mathrm{OH}$ & 39010 & 3-Jan-11 & 24-Jan-11 & $15-F e b-11$ & 28-Feb-11 & 2 & 4 \\
\hline 5 & $\mathrm{OH}$ & 39090 & 3-Jan-11 & 24-Jan-11 & $15-F e b-11$ & 28-Feb-11 & 2 & 4 \\
\hline 5 & $\mathrm{OH}$ & 39130 & 3-Jan-11 & 24-Jan-11 & 15-Feb-11 & 28-Feb-11 & 2 & 4 \\
\hline 5 & $\mathrm{PA}$ & 42010 & 3-Jan-11 & 24-Jan-11 & 15-Feb-11 & 28-Feb-11 & 3 & 4 \\
\hline 5 & $\mathrm{PA}$ & 42050 & 3-Jan-11 & 24-Jan-11 & 15-Feb-11 & 28-Feb-11 & 2 & 4 \\
\hline 5 & $\mathrm{PA}$ & 42080 & 3-Jan-11 & 24-Jan-11 & $15-$ Feb-11 & 28-Feb-11 & 2 & 4 \\
\hline 5 & PA & 42140 & 3-Jan-11 & 24-Jan-11 & 15-Feb-11 & 28-Feb-11 & 2 & 4 \\
\hline 5 & SD & 46100 & 3-Jan-11 & 24-Jan-11 & 15-Feb-11 & 28-Mar-11 & 2 & 4 \\
\hline 5 & TN & 47050 & 3-Jan-11 & 24-Jan-11 & $15-F e b-11$ & 28-Feb-11 & 2 & 2 \\
\hline 6 & IL & 17405 & 10-Jan-11 & 31-Jan-11 & 22-Feb-11 & 7-Mar-11 & 3 & 4 \\
\hline 6 & MI & 26090 & 10-Jan-11 & 31-Jan-11 & 22-Feb-11 & 7-Mar-11 & 3 & 4 \\
\hline 6 & $\mathrm{MN}$ & 27100 & 10-Jan-11 & 31-Jan-11 & 22-Feb-11 & 7-Mar-11 & 4 & 7 \\
\hline 6 & $\mathrm{MN}$ & 27200 & 10-Jan-11 & 31-Jan-11 & 22-Feb-11 & 7-Mar-11 & 2 & 4 \\
\hline 6 & $\mathrm{MN}$ & 27300 & 10-Jan-11 & 31-Jan-11 & 22-Feb-11 & 28-Mar-11 & 2 & 4 \\
\hline 6 & $\mathrm{MN}$ & 27400 & 10-Jan-11 & 31-Jan-11 & 22-Feb-11 & 7-Mar-11 & 1 & 4 \\
\hline 6 & $\mathrm{MN}$ & 27710 & 10-Jan-11 & 31-Jan-11 & 22-Feb-11 & 7-Mar-11 & 2 & 4 \\
\hline 6 & $\mathrm{MN}$ & 27900 & 10-Jan-11 & 31-Jan-11 & 22-Feb-11 & 7-Mar-11 & 1 & 5 \\
\hline 6 & NY & 36070 & 10-Jan-11 & 31-Jan-11 & 22-Feb-11 & 7-Mar-11 & 3 & 4 \\
\hline 6 & NY & 36153 & 10-Jan-11 & 31-Jan-11 & 22-Feb-11 & 21-Mar-11 & 2 & 3 \\
\hline 6 & $\mathrm{OH}$ & 39080 & 10-Jan-11 & 31-Jan-11 & 22-Feb-11 & 7-Mar-11 & 2 & 4 \\
\hline 6 & $\mathrm{OH}$ & 39120 & 10-Jan-11 & 31-Jan-11 & 22-Feb-11 & 7-Mar-11 & 2 & 4 \\
\hline 6 & $\mathrm{OH}$ & 39150 & 10-Jan-11 & 31-Jan-11 & 22-Feb-11 & 7-Mar-11 & 3 & 3 \\
\hline 6 & $\mathrm{PA}$ & 42020 & 10-Jan-11 & 31-Jan-11 & 22-Feb-11 & 7-Mar-11 & 3 & 3 \\
\hline 6 & $\mathrm{PA}$ & 42072 & 10-Jan-11 & 31-Jan-11 & 22-Feb-11 & 7-Mar-11 & 3 & 3 \\
\hline 6 & $\mathrm{PA}$ & 42130 & 10-Jan-11 & 31-Jan-11 & 22-Feb-11 & 7-Mar-11 & 2 & 4 \\
\hline 6 & $\mathrm{PA}$ & 42152 & 10-Jan-11 & 31-Jan-11 & 22-Feb-11 & 7-Mar-11 & 2 & 4 \\
\hline 6 & WI & 55700 & 10-Jan-11 & 31-Jan-11 & 22-Feb-11 & 7-Mar-11 & 3 & 4 \\
\hline 6 & WY & 56100 & 10-Jan-11 & 31-Jan-11 & 22-Feb-11 & 7-Mar-11 & 2 & 3 \\
\hline 7 & $\mathrm{AL}$ & 1600 & 20-Jun-11 & 11-Jul-11 & 5-Aug-11 & 22-Aug-11 & 2 & 4 \\
\hline 7 & $\mathrm{AZ}$ & 4200 & 13-Jun-11 & 5-Jul-11 & 29-Jul-11 & 15-Aug-11 & 2 & 4 \\
\hline 7 & FL & 12084 & 20-Jun-11 & 11-Jul-11 & 5-Aug-11 & 22-Aug-11 & 2 & 4 \\
\hline 7 & FL & 12150 & 27-Jun-11 & 18-Jul-11 & 12-Aug-11 & 29-Aug-11 & 1 & 4 \\
\hline
\end{tabular}

